\title{
Neural Principles Underlying Learning and Memory in Drosophila melanogaster
}

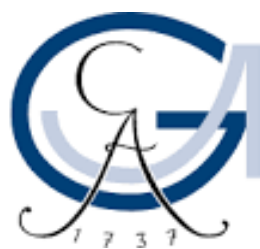 \\ Dissertation for the award of the degree \\ "Doctor rerum naturalium" \\ of the Georg-August-Universität Göttingen
}

within the doctoral program "Sensory and Motor Neuroscience", Göttingen Graduate School for Neurosciences, Biophysics, and Molecular Biosciences (GGNB)

submitted by

Clare Elizabeth Hancock

From Eastbourne, United Kingdom

Göttingen, 2021 


\section{Thesis Committee:}

\section{Prof. Dr. André Fiala}

(Department of Molecular Neurobiology of Behaviour, Johann-Friedrich-Blumenbach Institute for Zoology and Anthropology, Georg-August University, Göttingen)

\section{Dr. Camin Dean}

(Department of Synaptic Dysfunction, Deutsches Zentrum für Neurodegenerative Erkrankungen (DZNE), Berlin. Formerly: Department of Trans-synaptic Signaling, European Neuroscience Institute Göttingen (ENI), Göttingen)

\section{Prof. Dr. Gregor Bucher}

Department of Evolutionary Developmental Genetics, Johann-Friedrich-Blumenbach Institute for Zoology and Anthropology, Georg-August University, Göttingen)

\section{Members of the Examination Board:}

Reviewer: Prof. Dr. André Fiala

(Department of Molecular Neurobiology of Behaviour, Johann-Friedrich-Blumenbach Institute for Zoology and Anthropology, Georg-August University, Göttingen)

\section{Second Reviewer: Dr. Camin Dean}

(Department of Synaptic Dysfunction, Deutsches Zentrum für Neurodegenerative Erkrankungen (DZNE), Berlin)

\section{Dr. Jan Clemens}

(Department of Neural Computation and Behaviour, European Neuroscience Institute Göttingen (ENI), Göttingen)

\section{Prof. Dr. Ralf Heinrich}

(Department of Cellular Neurobiology of Behaviour, Johann-Friedrich-Blumenbach Institute for Zoology and Anthropology, Georg-August University, Göttingen)

\section{Prof. Dr. Carolin Wichmann}

(Molecular Architecture of Synapses Group, Institute of Auditory Neuroscience, University Medical Centre, Göttingen) 




\section{Table of Contents}

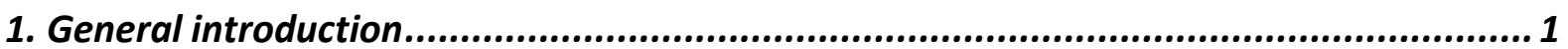

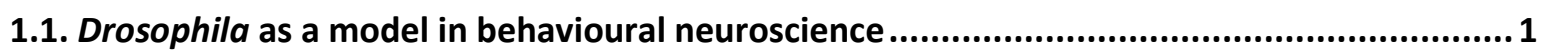

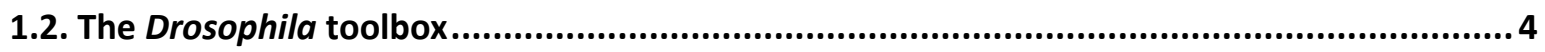

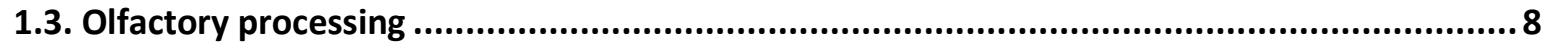

1.4. The Drosophila mushroom body - functional dissection of a learning centre ..................... 11

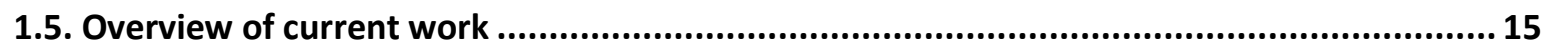

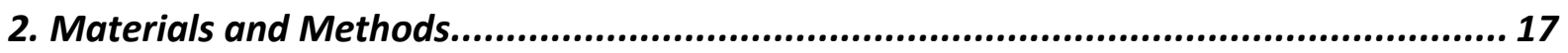

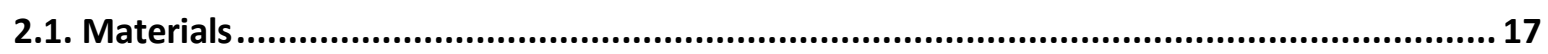

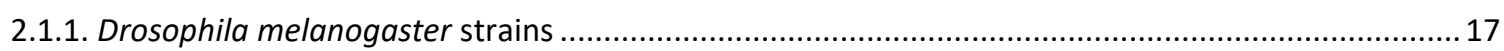

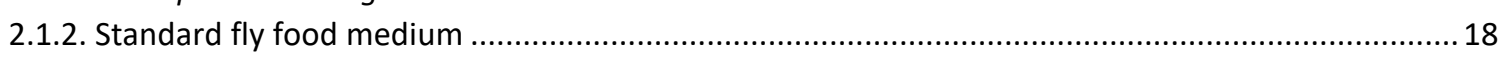

2.1.3. Chemicals

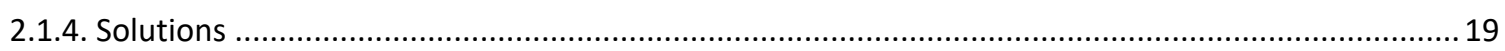

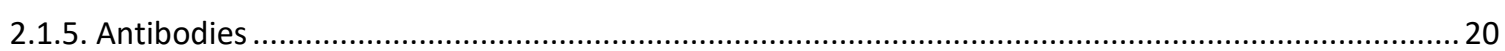

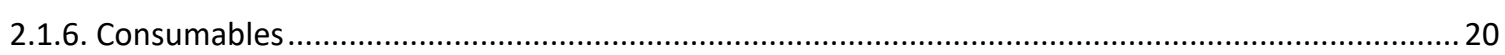

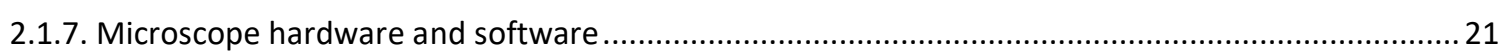

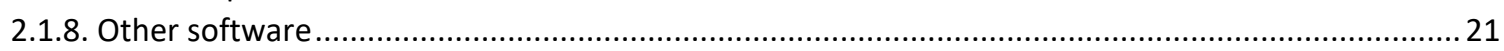

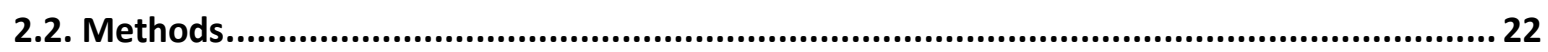

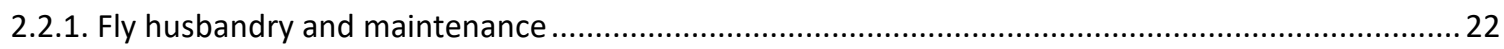

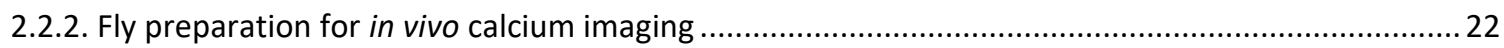

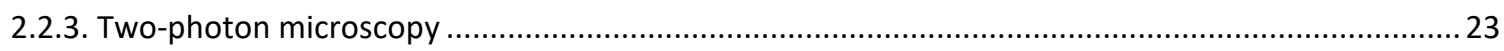

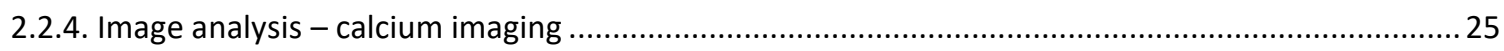

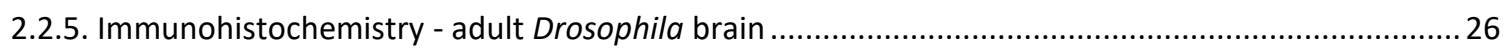

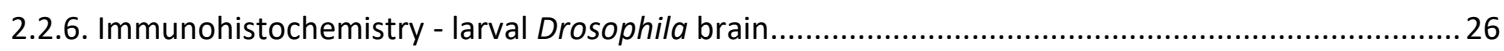

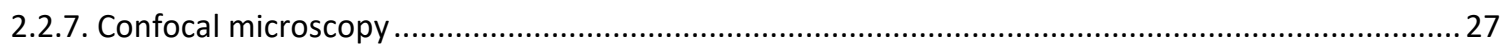

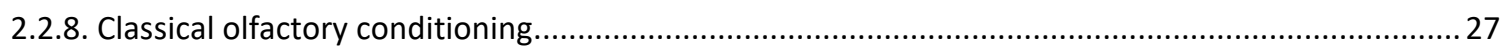

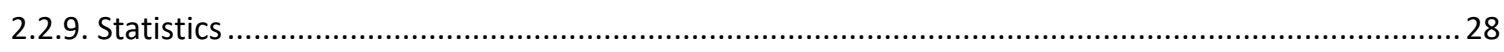

3. Visualisation of Learning-Induced Plasticity at the MBON Postsynapse....................... 31

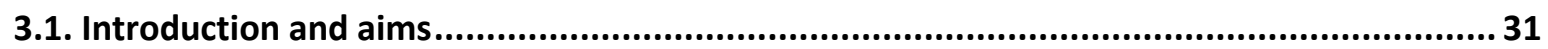

3.2. Manuscript - In vivo Calcium Imaging of Learning-Induced Synaptic Plasticity in Drosophila

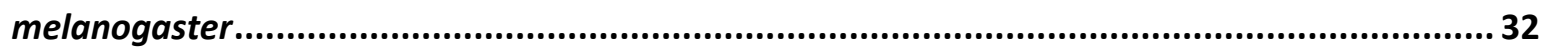

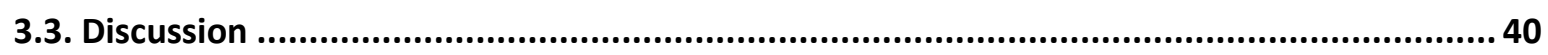

4. Learning-induced Modulation of Mushroom Body Output Neuron Postsynapses........ 41

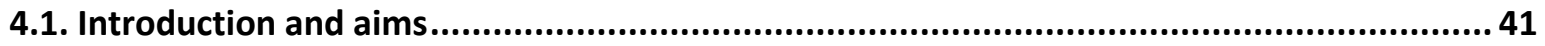

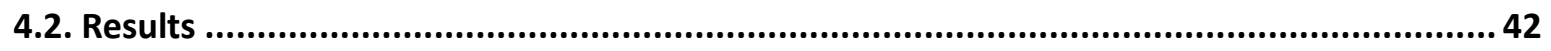

4.2.1. Localisation of homer-GCaMP to the mushroom body output neuron post-synapse.......................42

4.2.2. $\mathrm{MBONs}$ of the $\mathrm{MB} \mathrm{Y}$-lobe receive heterogenous odour drives ....................................................43

4.2.3. Aversive associative conditioning leads to suppression of $\mathrm{MBON}-\gamma 1$ at the postsynapse ................45

4.2.4. MBONs- $\gamma 2-5$ show no detectable postsynaptic memory trace ...................................................4 47

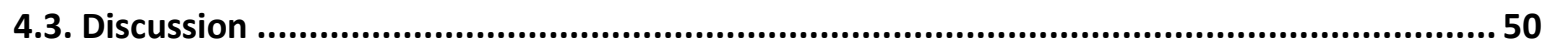




\section{Visualisation of Learning-Induced Modulation of Odour-Evoked Activity at Kenyon Cell}

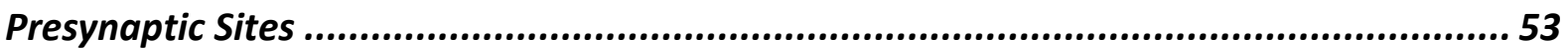

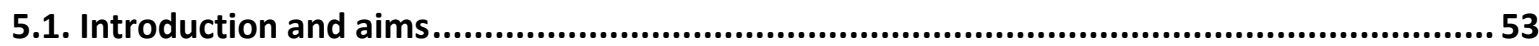

5.2. Manuscript - Visualization of Naïve and Learned Odor Representations Using in vivo Calcium Imaging and Immunohistochemical Bouton Mapping of Single Drosophila Mushroom

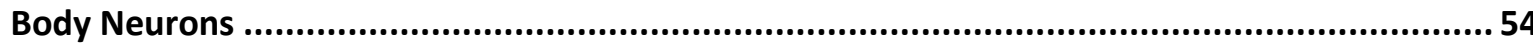

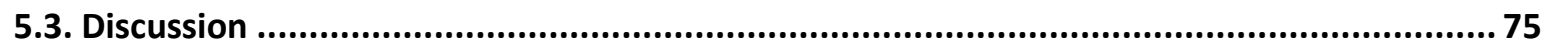

6. Visualization of a Distributed Synaptic Memory Code in the Drosophila Mushroom Body

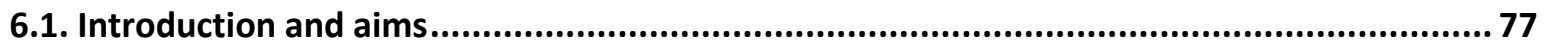

6.2. Manuscript - Visualization of a Distributed Synaptic Memory Code in the Drosophila Brain

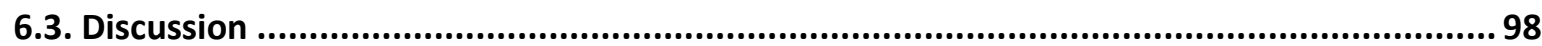

7. Functional and Anatomical Investigation of MB-CP1 Neurons.............................. 101

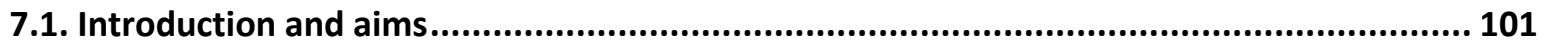

7.2. Results

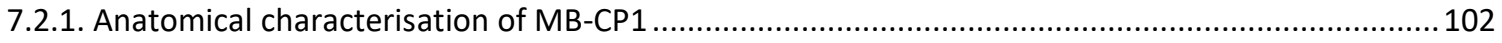

7.2.2. MB-CP1 neuron output is not required for learning and short-term memory ................................ 104

7.2.3. MB-CP1 neurons exhibit a narrow and hard-wired odour tuning profile........................................ 105

7.2.4. The influence of hunger and sex on MB-CP1 neuron odour tuning .............................................. 109

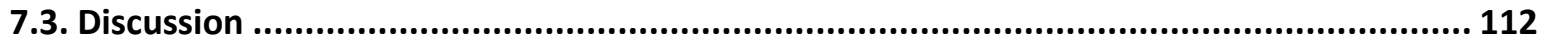

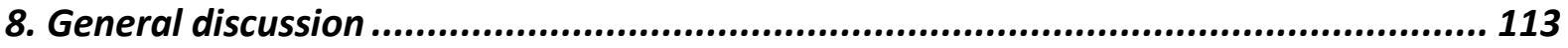

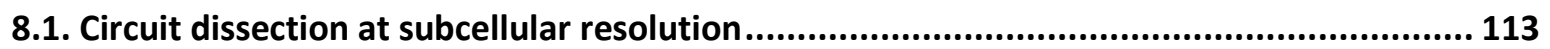

8.2. Mushroom body compartmentalisation: implications for associative learning ................. 115

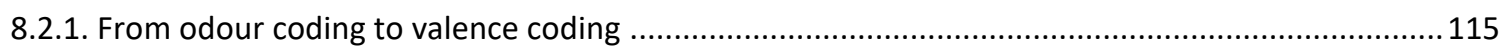

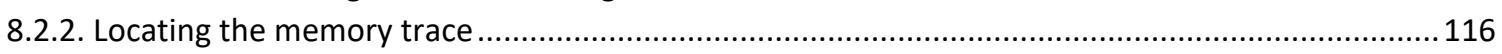

8.3. Investigation of factors influencing naïve odour tuning ................................................ 120

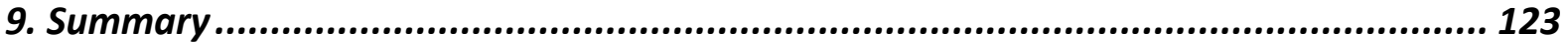

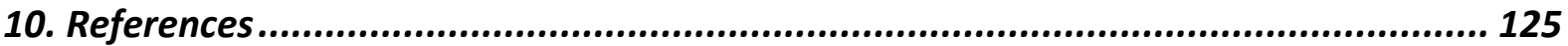

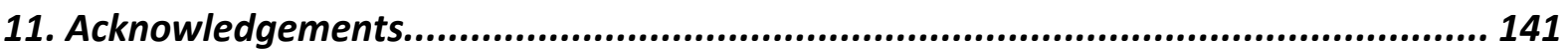

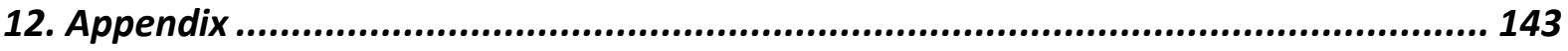

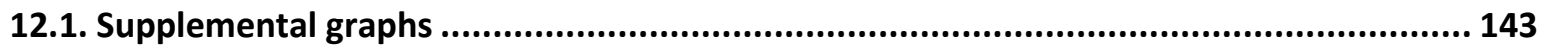

12.1.1 MBONs- $\gamma 2-5$ show no detectable postsynaptic memory trace ............................................... 143

12.2. Supplemental material - Visualization of a Distributed Synaptic Memory Code in the Drosophila Brain

Supplemental Figure S2 from the publication "Visualization of a Distributed Synaptic Memory Code in the Drosophila Brain" ........................................................................................................................... 147 Supplemental Figure S6 from the publication "Visualization of a Distributed Synaptic Memory Code in the

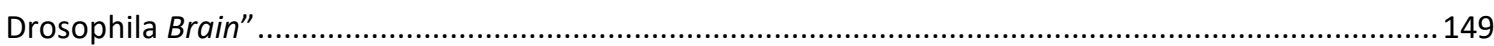


Curriculum vitae.

151 



\section{General introduction}

How organisms perceive and interact with their environment is a key determinant of their survival. Indeed, from simple bacteria to the highest vertebrates, the detection, processing, and responsiveness to external stimuli is fundamental. Although the complexity of these processes varies widely across taxa, the principle remains the same - if an organism detects the presence of a particularly noxious or attractive substance in its vicinity, it benefits from responding in an appropriate and timely manner such that those things that threaten life are avoided and those that preserve it are sought out.

However, the more complex the ecological niche an organism occupies, the more sophisticated must be the biological tools at its disposal. It follows then, that evolution has given rise to increasingly refined sensory systems, which feed information into centralised nervous systems capable of processing simultaneous, multimodal inputs. The initiation of complex behaviours - such as locating and navigating toward food sources or identifying the presence of a predator and seeking safety - rely not only on the detection of these sensory inputs, but more importantly on the prior knowledge that those sensations are predictive of positive or negative outcomes. This prior knowledge is, broadly speaking, either innate or acquired. The work presented here will largely focus on the latter - using the experimentally versatile model Drosophila melanogaster to investigate the neural principles underlying the mechanisms by which sensory stimuli acquire behaviourally relevant meaning.

\subsection{Drosophila as a model in behavioural neuroscience}

The fruit fly, Drosophila melanogaster, presents researchers with a model system that walks the line perfectly between biological simplicity and behavioural complexity. Therein, though equipped with a relatively modest central nervous system, Drosophila are able to execute a plethora of wellcharacterised and ecologically interesting behaviours that can, due to the easy rearing and handling of the flies, be analysed in a high-throughput and reproducible manner.

Indeed, fruit flies face the same basic struggles as any animal - that is, surviving against adverse environmental conditions and predation to find food and mates that ensure the production of viable offspring. As such, they have often been recruited by biologists who have designed experimental apparatuses to investigate the genetic, molecular, and systemic processes that underly the fulfilment of these universal drives. Such endeavours began over a century ago, with the design of simple behavioural assays to test the responses of flies to different sensory stimuli - for example, light 
(Carpenter, 1905) or smell (Barrows, 1907). These early experiments were initially based on anecdotal observations of Drosophila behaviour - with Carpenter noting that the flies he stored in bottles with rotten bananas and apples appeared to aggregate on the side of the bottle exposed to sunlight through a window, and Barrows noticing that flies in his laboratory had a predilection for open alcohol bottles. By then taking these observed behaviours and analysing them under controlled conditions, work like this paved the way for the development of more complex behavioural analyses.

This development is helped by the fact that many behaviours that can be observed in Drosophila are highly stereotyped. For example, social behaviours such as fighting and courtship both consist of several individual actions - often carried out in a specific sequence or under specific circumstances that can be qualitatively and quantitatively evaluated. Fighting between male flies, for instance, can be observed in the form of a few distinct actions - so-called "wing-threats" (during which the male raises and spreads its wings in the direction of an adversary), charging, and boxing - each of which are easily identifiable and specific to aggressive interactions (Chen et al., 2002; Dow \& von Schilcher, 1975). Similarly, courtship is likewise stereotyped and well-suited for experimental dissection comprising an intricate step-wise progression from locating a potential mate, "singing" to her via wing vibrations and tapping her with his forelegs, to eventually attempting copulation (Bastock \& Manning, 1955; Sturtevant, 1915). This stereotypy in behavioural expression makes for a favourable base upon which the underlying control mechanisms of those behaviours can be explored. In more contemporary work, this has also meant that automated video tracking software can be used to detect and quantify these specific actions (Dankert et al., 2009; Reza et al., 2013).

Each of the examples given above are innately driven behaviours, which are inborn and not gained through experience. However, another strength of Drosophila as a model in behavioural studies is their ability to adapt their behaviour dependent on prior experience. The most commonly explored case of this is in studies of associative learning, which can be divided broadly into either classical (Pavlovian (Pavlov, 1927)) conditioning or operant conditioning. In the former, an animal learns to associate a previously unremarkable, neutral sensory stimulus with one that confers an innately positive or negative outcome. This type of conditioning in Drosophila is usually investigated by teaching flies to associate an olfactory stimulus with either sugar (which is very attractive) or the receipt of electric shocks to the legs and body (which is very aversive). First established in the 1980s (Tempel et al., 1983; Tully \& Quinn, 1985), the conditioning paradigm to induce these associations is relatively simple to carry out: flies are confined to a tube that is lined with either an electrical grid or filter paper soaked with sugar water, and into that tube a specific odorant is delivered. Here, the delivery of electric shock or sucrose act as unconditioned stimuli (US) as they alone evoke a behavioural response (unconditioned response, UR), and the odorant is deemed the conditioned 
stimulus $+(\mathrm{CS}+)$ as it is then associated with the US such that later presentation of the CS+ alone evokes a conditioned response (CR) resembling the UR. In most cases, a differential conditioning paradigm is used - meaning flies are exposed to an additional odour in the absence of the US (conditioned stimulus -, CS-). When flies are then presented with a choice between the CS+ odour and the CS- odour in a T-maze set-up, the flies that learned the association will show either an attraction or an aversion to the CS+ (in the case of sugar or electric shock association, respectively). By quantifying the number of flies that show this $C R$, experimenters have a simple readout for learning performance.

Operant (or instrumental) conditioning, first described by B.F. Skinner (Skinner, 1948; Skinner, 1938), on the other hand, is not based on associations of sensory stimuli with one another, but rather the association of an animal's behaviour with positive or negative outcomes. In Drosophila, for example, by exposing flies to a noxious heat stimulus if they enter a specific area of an arena or orient their body at specific angles during flight simulation tasks (Mariath, 1985; Wustmann et al., 1996), one can train the animal to associate the preceding behaviour with punishment and therefore reduce the likelihood that that behaviour is repeated in the future.

It is also interesting to note that even the very stereotyped, innate behaviours discussed previously are subject to experience-dependent modification. For example, Siegel and Hall (Siegel \& Hall, 1979) showed that males that attempt - and fail - to court females that had already mated show a conditioned preference for courting virgin females in subsequent observations. Given that mated and virgin female flies give out distinct sensory cues, this suggests that the males learn to associate those cues from mated females as predictive of rejection and thus suppresses their drive to court them in the future (Ejima et al., 2005; Griffith \& Ejima, 2009). Similarly, flies seem to also learn from previous aggressive interactions, with males that lost in previous fights appearing to become more submissive and more likely to lose again in subsequent bouts (Trannoy et al., 2016; Yurkovic et al., 2006).

The experimental simplicity and versatility of the behavioural assays described here makes Drosophila an ideal model for the dissection of the mechanisms underlying behavioural expression. It is also important to note that, at the same time as Carpenter and Barrows were starting to observe simple behaviours in Drosophila at the turn of the $20^{\text {th }}$ century, geneticist T.H. Morgan and his colleagues were also making significant strides in the study of inheritance using the same model organism (Morgan, 1911; Morgan, 1910). Morgan's discovery of the mutant gene white, which results in flies with white rather than red eyes, and the following characterisation of the inheritance of that gene through subsequent generations, signified a huge step in our collective understanding of the fundamentals of genetics. This work also established Drosophila as an invaluable tool in this field, and 
led ultimately to the routine use of Drosophila in behavioural genetics in the latter half of the $20^{\text {th }}$ century.

This expansion - from pure genetics to the genetics of behaviour - is well exemplified by the work of Seymour Benzer and his colleagues who, in the 1970s, used random mutagenesis to isolate genes directly involved in specific behaviours: for example, the role of the period gene in the maintenance of circadian rhythms (Konopka \& Benzer, 1971), and the role of the dunce gene in associative learning (Dudai et al., 1976). These studies, and others like them of the time, utilised the relatively advanced knowledge of Drosophila genetics to start to draw connections between genes and behavioural expression. The half-century since these experiments has been characterised by the expansion of the genetic tools, and therefore experimental approaches, available for Drosophila research.

\subsection{The Drosophila toolbox}

While random mutagenesis screens have provided vital insights into the genes underlying behaviour in Drosophila, the genetic tools that have been developed more recently have given rise to more precise methods. This has been enabled by the generation of genetic constructs that allow for the expression of transgenes in specific populations of cells (or, more recently, single cells). The most commonly used of these expression systems are the Gal4/UAS system and the LexA/LexAop system (Brand \& Perrimon, 1993; Lai \& Lee, 2006). Both are based on the introduction of a transcription factor (Gal4 or LexA) and an enhancer sequence to which it binds (UAS [Upstream Activation Sequence] or LexAop [LexA operator]), placed upstream of a transgene of interest (Figure 1.1., A). By placing the chosen transcription factor under the control of a tissue or cell-type specific promotor, the experimenter has spatial control over transgene expression. Further refinement of these tools has led to more precise spatial control, for example by driving the expression of the two domains of the Gal4 protein (the activation domain and DNA-binding domain) under control of two different enhancers (Figure 1.1., B). This approach - called split Gal4 - results in a narrower expression pattern, as only in cells in which the two promoters show overlapping activity will a functional Gal4 be produced (Luan et al., 2006). Spatial restriction of Gal4 expression can also be achieved by the co-expression of the Gal4 repressor, Gal80, in a specific sub-population of cells that one wishes to exclude (Figure 1.1., C)(Lee \& Luo, 1999). 


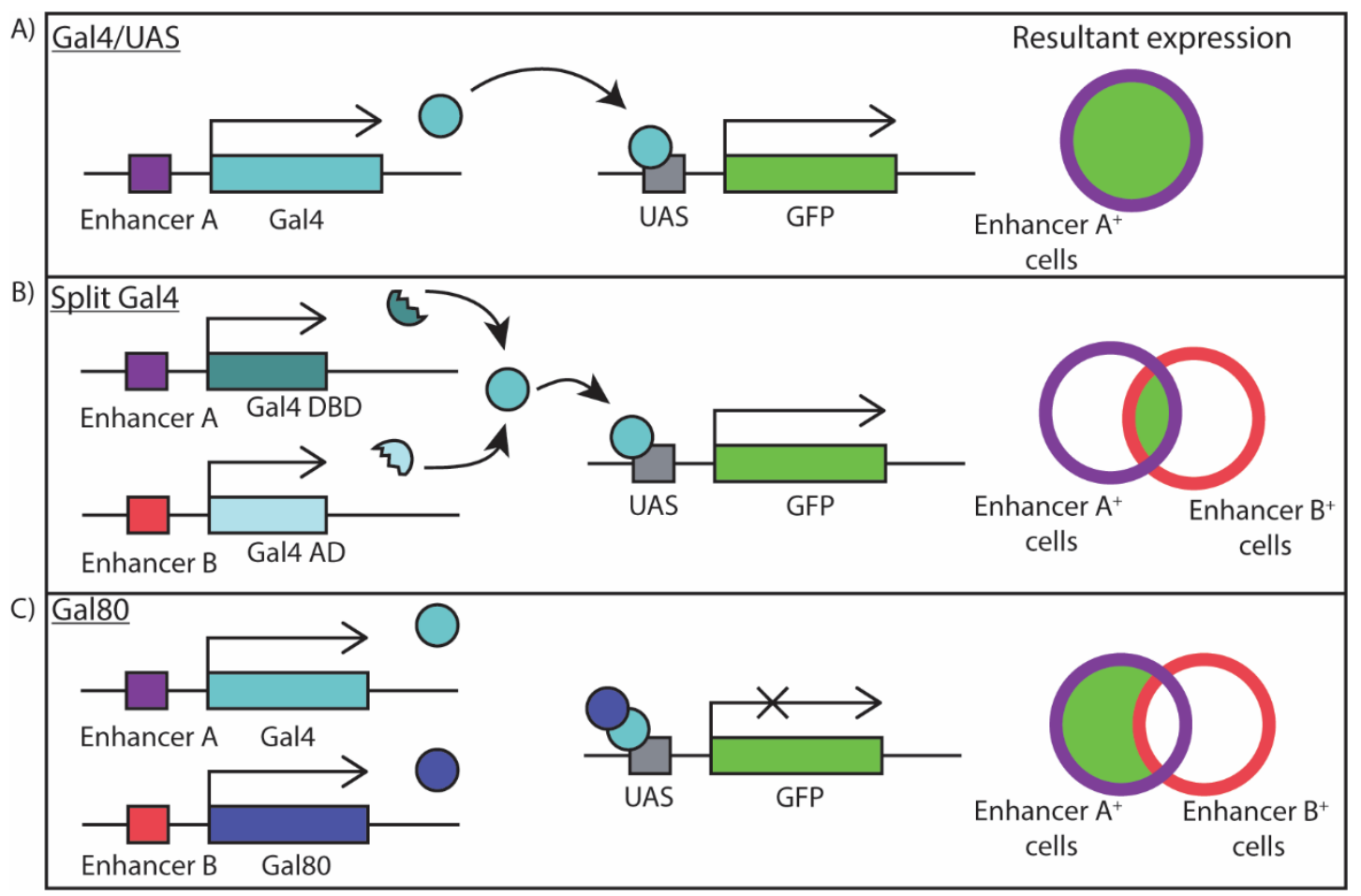

Figure 1.1.: Gal4-based transgene expression systems in Drosophila. (A) Yeast-derived Gal4 transcription factor is placed downstream of a tissue or cell type specific enhancer. Gal4 then binds to Upstream Activation Sequences (UAS) upstream of transgene of interest (e.g., in this case GFP). (B) The two domains of Gal4 are separated and placed under the control of separate enhancers, leading to a more restricted expression pattern. (C) Use of the Gal4 repressor, Gal80, under control of a second enhancer to eliminate a subset of cells from transgene expression.

By the spatially restricted expression of transgenes that disrupt or enhance endogenous genes or proteins, the role of specific genes and their products can be investigated in the context of defined populations of neurons in the brain. This has facilitated more in-depth examination of the neuronal circuits and intracellular processes underlying behaviours such as regulation of circadian rhythms by cell-type specific rescue of the period gene (Grima et al., 2004) or specific silencing of circadian pacemaker neurons (Nitabach et al., 2002); feeding by localisation and manipulation of gustatory receptors (Scott et al., 2001) and neuropeptides (AI-Anzi et al., 2010) that mediate food seeking and consumption; and learning and memory by selective silencing of specific subsets of mushroom body neurons (Dubnau et al., 2001) or the spatially precise rescue of the rutabaga gene (Zars et al. 2000a). Each of these studies utilised the spatially restricted expression of transgenes to precisely dissect the specific cells involved in these behaviours.

When doing so, it is important to note that this exogenous expression always has the potential to confer off-target effects that may influence behaviour - for example, down-regulation of structural proteins involved in synapse formation may cause defects in synaptic plasticity and thus learning 
behaviour, but it is equally likely that this defect is a result of maldevelopment of neurons before any behavioural assays have been conducted. Therefore, the development of the TARGET (Temporal and Regional Gene Expression Iargeting; McGuire et al. 2003) method was another vital step in the development of tools for the examination of gene-behaviour relationships in Drosophila. This method utilises a temperature-sensitive form of the Gal4 repressor, Gal80 (Gal80 $\left.{ }^{\text {ts }}\right)$, to control the induction of Gal4 (and thus UAS) expression via a timely shift in the rearing temperature of experimental flies. By adding this element of temporal control to an already spatially precise expression system, more clear-cut conclusions can be drawn regarding the roles of specific genes.

Another key addition to the Drosophila toolkit has been those that allow the direct activation or silencing of neurons. This is most commonly achieved using genetically encoded light-sensitive ion channels - a technique called optogenetics (Fiala et al., 2010; Suh et al., 2007). When flies are exposed to light of a specific wavelength, these channels open and allow movement of ions through the cell membrane. Dependent on the specific channel expressed, these ions may be cations or anions and cause activation (membrane depolarisation) or silencing (membrane hyperpolarisation), respectively. A similar effect can be achieved using temperature sensitive proteins. By expressing the temperature sensitive dynamin variant shibire ${ }^{t s}$ in neurons of interest, one can induce a depletion of synaptic vesicles via a temperature increase to $29^{\circ} \mathrm{C}$ such that neurotransmitter release is temporarily inhibited (Kitamoto, 2001). Conversely, one can use the same temperature shift protocol to induce depolarisation of neurons expressing temperature sensitive transient receptor potential channel dTRPA1 (Hamada et al., 2008). By driving the expression of transgenes encoding these proteins in neurons of interest and observing the behavioural consequences of their artificial activation or silencing, experimenters can identify the neurons whose activity is necessary and/or sufficient for the expression of a given behaviour.

As well as artificially controlling the activity of neurons, it is also valuable to be able to observe their activity under different experimental conditions. The predominant methodology for this over the last century, across organisms, has been electrophysiological recording - particularly in the field of invertebrate learning and memory research. For example, foundational studies from Eric Kandel and colleagues were instrumental in the establishment of electrophysiological recordings in the marine snail, Aplysia californica, during the induction of different forms of synaptic facilitation or depression (Carew et al., 1981; Kandel et al., 1967; Kandel \& Tauc, 1965). Not only did these studies contribute to our collective knowledge of synaptic plasticity and its underlying mechanisms, but they also unequivocally demonstrated the value of invertebrate model systems in studies of cognitive functions that until then were predominantly confined to higher vertebrates. 
While the size and easy identification of neurons in Aplysia make it an attractive model for monitoring of neuronal activity, with the development of the genetic tools discussed above, Drosophila has become a more versatile and attractive model. One of the key advantages of these tools is that they allow for more tailored and less invasive experimentation. For instance, in Drosophila it is not necessary (or, indeed, possible in most cases) to identify and access neurons of interest with microelectrodes for monitoring. Rather, by using the genetic expression systems described above (e.g., the Gal4/UAS system), specific cells can be targeted, and their activity monitored by the expression of exogenous indicators of neuronal activity - such as indicators of intracellular calcium concentration. This principle forms the basis of the main methodology utilised in this work - in vivo functional imaging using genetically encoded calcium indicators (GECls).

This technique - also broadly called calcium imaging - utilises the expression of transgenes encoding a protein that emits fluorescence dependent on intracellular calcium ion concentration. The most commonly used of these is GCaMP, a fusion of GFP and the calcium-binding protein calmodulin, first demonstrated by Nakai, Ohkura, and Imoto (2001). Multiple subsequent iterations of GCaMP have been developed over the years that have improved signal-to-noise ratio, introduced variable calcium binding kinetics, broader dynamic ranges, and increased photostability (Akerboom et al., 2012; Chen et al., 2013; Dana et al., 2019; Nakai et al., 2001; Tian et al., 2009). Each shares the same broad mechanism of action, whereby, upon calcium binding to the calmodulin component, a conformational change occurs to the GCaMP such that GFP fluorescence is enhanced. Upon depolarisation, intracellular calcium increases rapidly - due to the opening of voltage-gated calcium channels, and release from intracellular stores - leading to more calcium binding to GCaMP, and an increase in GFP fluorescence that can be detected and quantified as a proxy for neuronal activity.

The present work benefited by further spatially restricting the expression of GECls. In sections 6 and 7 , we incorporate the technique of mosaic analysis with a repressible cell marker (MARCM; Wu and Luo 2006) to reduce the number of neurons expressing our GECI. This technique uses heat-shock induced, flippase (FLP)/flippase recognition target (FRT)-mediated recombination to stochastically "flip-out" the Gal4 repressor Gal80 in subpopulations of Gal4-expressing neurons to result in a sparser labelling of neurons. This was used to visualise activity in single Kenyon cell axons in the mushroom body, which is not possible using Gal4-driven approaches alone due to the dense overlapping and intertwining of the axons making anatomical and functional differentiation of neurons impossible.

Furthermore, in sections 3 and 4, we utilise a GECI that localises specifically to the postsynaptic compartments of neurons via fusion of GCaMP to the postsynaptically targeted Homer protein (Pech et al., 2015). This subcellular restriction of calcium detection allows for dissection not only of the 
neurons that are activated under certain experimental circumstances, but also the finer spatial dynamics of calcium influx. Similar calcium indicators have also been generated that are localised to presynaptic sites, via linkage to Synaptophysin (Pech et al., 2015).

Simultaneous developments in microscopy techniques have also driven forward the field of calcium imaging, improving spatial and temporal resolution such that changes in intracellular calcium can be accurately and precisely visualised in single neurons, including in the living animal. These technological advances have benefited not only the functional imaging of the nervous system, but also its anatomical dissection. Using electron microscopy and Al-assisted image processing, large parts of the Drosophila brain have been scanned at high resolution and many thousands of neurons reconstructed in three dimensions (Li et al., 2020; Scheffer et al., 2020; Takemura et al., 2017). This technique also allowed for the identification of putative sites of synaptic connections between neurons, providing some insight into possible patterns of neuronal connectivity in the brain that can be used as a starting point in identifying potential neuronal circuits involved in behaviour.

The tools described here make Drosophila a favourable model in which to study the neural basis of behaviour. Indeed, these tools and the studies they have facilitated have helped to further our knowledge of sensory systems and how animals use them to guide their actions. This is particularly true of the Drosophila olfactory system.

\subsection{Olfactory processing}

The sense of smell is fundamental in guiding behaviour, conveying a continuous stream of information about the external environment to the brain for processing. In Drosophila, this starts at the sensilla on the antennae and maxillary palps, where odorant particles bind to specific odorant receptors (ORs) expressed in olfactory receptor neuron (ORN) dendrites. Typically, each ORN expresses one OR type (out of the 62 ORs identified in Drosophila (Robertson et al., 2003), plus the odorant receptor coreceptor, Or83b (also known as ORCO; Larsson et al., 2004; Vosshall et al., 2000). The axons of the ORNs extend into the antennal lobe, where ORNs that express the same receptor converge to form glomerular structures (Vosshall et al., 2000)(Figure 1.2., A). When an odour is encountered, a combination of ORNs is activated to produce an early neural perception of the odour. This is relayed to higher brain regions by olfactory projection neurons (OPNs), which are broadly classed as either uniglomerular (164 OPNs) or multiglomerular (184 OPNs) depending on if their dendrites innervate one glomerulus in the antennal lobe or several, respectively (Bates et al., 2020). A third neuron type - the local interneurons (LNs) - also influences the activity of OPNs, by exerting (primarily) inhibitory 
inputs to both the ORNs and the OPNs in the antennal lobe, as well as other LNs (Ng et al., 2002; Wilson et al., 2004).

From there, the OPNs project to the lateral horn (LH), with a subset also projecting to the mushroom body (MB) calyx. Broadly speaking, the former brain region is categorised as mediating innate behaviour (Belle \& Heisenberg, 1994; Jefferis et al., 2007), whereas the latter is believed to predominantly mediate learned behaviour (Belle \& Heisenberg, 1994; Heisenberg et al., 1985; McGuire et al., 2001). The neural circuits that form these brain regions have been the subject of many in-depth studies recently, due in large part to the availability of many thousands of split Gal4 driver lines that now allow for their anatomical and functional description, and also due to connectomics efforts that allow the tracing of single neurons through these regions (Aso et al. 2014a; Bates et al. 2020; Dolan et al. 2019). There appears to be some spatial organisation of OPN inputs to these brain regions - for example, there seems to be a concentric organisation of inputs to the MB calyx, whereas spatial OPN input patterns to the LH are less geometrically organised but more stereotypic between individuals (Jefferis et al., 2007; Tanaka et al., 2004). Notably, OPNs that respond to food-related odours seem to extend their axons to similar subregions within the MB calyx, as well as the LH (Bates et al., 2020). In the LH, this is also the case for OPNs responsive to pheromones (Jefferis et al., 2007). Considering such correlations have not been observed for other types of OPNs, this may imply a differential odour processing mechanism for ethologically relevant odours in these higher brain regions.

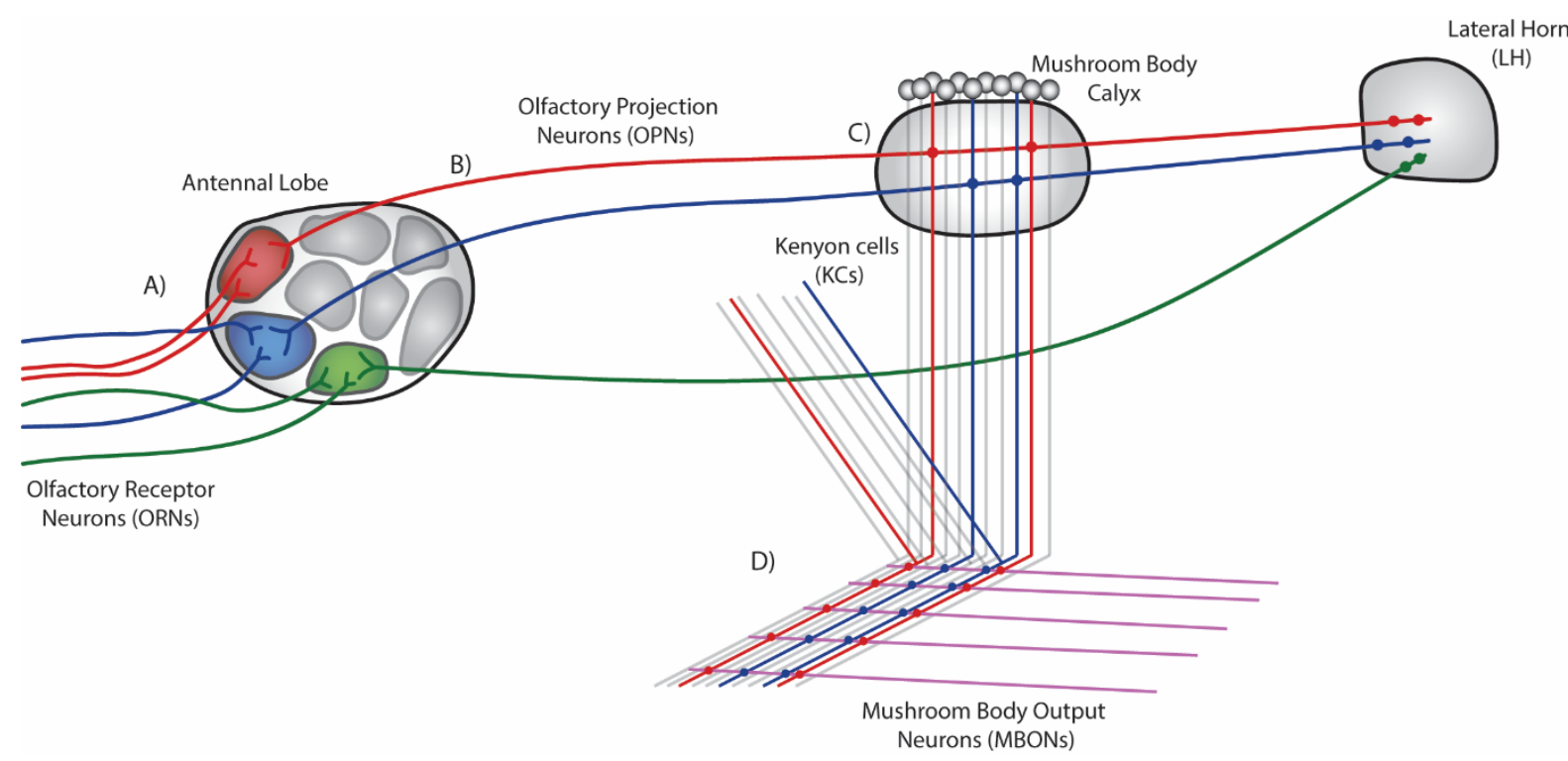

Figure 1.2.: Odour processing pathways in the Drosophila brain. (A) Odours are first detected by Olfactory Receptor Neurons (ORNs), expressing specific odorant receptors, which project into the antennal lobe to form glomerular structures. (B) ORNs synapse onto Olfactory Projection Neurons (OPNs) in the antennal lobe glomeruli. OPNs then project to higher brain regions such as the lateral 
horn and the mushroom body calyx. (C) In the calyx, OPNs synapse onto the mushroom body intrinsic Kenyon cells (KCs). This olfactory input leads to a sparse activation of $K C s$, the axons of which project out of the calyx to form the lobes of the mushroom body. (D) In the mushroom body lobes, mushroom body output neurons (MBONs) receive highly convergent olfactory input from the large population of $\mathrm{KCs}$, such that responses at this level become very broad.

The $\mathrm{MB}$ is formed of Kenyon cells (KCs), the dendrites of which reside in the calyx region and are postsynaptic to the OPNs (Figure 1.2., C). The KCs form claw-like structures around OPN presynaptic boutons, wherein each KC receives input from 5-7 randomised OPNs (Caron et al., 2013; Leiss et al., 2009). The intrinsic properties of the KCs are such that several active OPN inputs must be received in order for their spiking threshold to be reached, which, combined with the action of the inhibitory anterior paired lateral (APL) neuron, results in sparse KC odour-evoked activation (Lin et al., 2014). This sparseness appears to convey an advantage in the ability of the MB to encode odours in a way that odour identity can be discriminated based on the activity pattern across the KCs - providing a network structure well equipped to form olfactory-driven memories without generalising experiences across multiple odorants (Lin et al., 2014; Liu \& Davis, 2009; Pitman et al., 2011). Thus, the transformation of an odour representation from a broad array of OPNs to a sparse KC activation pattern in the MB that occurs here is a crucial step in olfactory processing in Drosophila.

The axons of the KCs form the lobes of the MB, connected to the calyx by the pedunculus. Each $M B$ is formed of approximately 2000 KCs (Aso et al., 2009; Aso et al., 2014a; Li et al., 2020), within which there are three main classes $-\alpha / \beta, \alpha^{\prime} / \beta^{\prime}$, and $\gamma$ - that are genetically and anatomically distinct (Aso et al., 2014a; Crittenden et al., 1998). Where the latter projects only horizontally toward the midline, the $\alpha / \beta$ and $\alpha^{\prime} / \beta^{\prime}$ type KCs both bifurcate to form vertical ( $\alpha$ and $\alpha^{\prime}$ ) and horizontal ( $\beta$ and $\beta^{\prime}$ ) lobes. These different $\mathrm{KC}$ types also have differing roles in the control of behaviour, particularly with regards to learning and memory formation, as will be discussed in the next section. All lobes of the MB are further subdivided into compartments, defined by the innervation patterns of MB extrinsic neurons the two main populations of which being dopaminergic neurons (DANs) and MB output neurons (MBONs)(Aso et al., 2014a).

The DANs that innervate the MB play an important role in behavioural control. For example, dopamine signalling in the MB has been linked to hunger-state dependent food-seeking behaviours (Landayan et al., 2018; Tsao et al., 2018), temperature preference behaviour (Bang et al., 2011), courtship (Kuo et al., 2015; Lim et al., 2018), and associative learning (Krashes et al., 2009; Liu et al., 2012; Schroll et al., 2006). There are eight main DAN clusters around the MB, two of which - the PPL1 and PAM clusters - project their axons into specific compartments of the MB lobes (Mao \& Davis, 2009), where they 
transmit modulatory signals to the KCs (and MBONs) dependent on internal and external contextual cues (such as the hunger state of the animal or the presence of rewarding or punishing stimuli). Indeed, the reward- or punishment-induced activity of DANs is considered to be the driving force behind associative olfactory learning.

MBONs are the primary downstream target of the $\mathrm{KCs}$, and also receive input from DANs. Where the OPN-to-KC level of the olfactory pathway is highly divergent (with 350 OPNs synapsing onto 2000 $\mathrm{KCS}$ ), the KC-to-MBON level represents a return to a more convergent connectivity. Per MB, there are 34 MBONs, which are divided into 21 different types dependent on the MB compartment their dendrites innervate (Aso et al., 2014a; 2014b). Like the DANs, the MBONs also have differing functions depending on this innervation pattern. Optogenetic experiments carried out by Aso et al. showed that the activation of selected MBONs results in approach or avoidance behaviour, and thus led to the conclusion that the MBONs are responsible for coding valence properties of stimuli (Aso et al., 2014b).

An additional class of so-called "atypical" MBONs was recently identified that display dendritic arbours that receive input not only from the $\mathrm{MB}$, but also in several distinct regions around the MB (Li et al., 2020), although these neurons have not yet been functionally examined.

From the mushroom body, the MBONs integrate inputs from hundreds of KCs (Figure 1.2., D) and relay this to further brain regions - such as the crepine, superior medial protocerebrum, and the lateral horn (Aso et al., 2014a; Li et al., 2020; Tanaka et al., 2008). Fundamentally, this integration across many KCs signifies a step in olfactory processing whereby the incident odour is no longer processed in terms of specific odour identity, but rather as a representation of that odour's valence. In the context of olfactory associative learning, the MBONs are placed perfectly to act as a readout of learned odourpunishment or odour-reward associations and to transmit updated valence information to motor control circuits to implement appropriate behaviour.

\subsection{The Drosophila mushroom body - functional dissection of a learning centre}

The predominant models for associative learning in insects all centre around the MB. This is based on decades of research that has sought to localise memory traces in the brain, with the first strong experimental evidence being shown by Menzel and colleagues in the honeybee, Apis mellifera (Erber et al., 1980; Menzel \& Erber, 1978), and Heisenberg and colleagues in Drosophila (Belle \& Heisenberg, 1994; Heisenberg et al., 1985). These studies used ablation of the MB, by specific cooling of the MB, or the chemical or genetic disruption of $\mathrm{MB}$ structure, to show that performance in associative learning tasks is drastically reduced without the proper functioning of the MB. Following this finding, 
more in-depth dissection has unveiled differential roles for the different lobes of the MB in different types of learning tasks. For example, the $\alpha$-lobes have been shown to be required for long-term memory (LTM) using the alpha-lobes-absent (ala) mutant (Pascual \& Préat, 2001) and the $\gamma$-lobe has been linked to short-term memory (STM) via spatially restricted rescue of rutabaga (rut) mutants and selective inhibition of neurotransmitter release (Isabel et al., 2004; Zars et al., 2000b). Two different types of anaesthesia resistant memory (ARM), with different temporal properties, have been shown to require synaptic output and dunce expression in distinct populations of MB cells, with short-term ARM requiring the $\alpha / \beta$ lobe, while long-term ARM requires the $\alpha^{\prime} / \beta^{\prime}$ lobe (Bouzaiane et al., 2015). In the current work, we are primarily interested in the processes underlying STM and so this will be discussed in detail.

During the classical conditioning paradigm used to induce STM in most Drosophila learning studies, presentation of an odour is temporally paired with either an electric shock or a sugar reward such that when flies encounter that odour again in a testing phase, they have either learned to avoid it or approach the odour, respectively (Tully \& Quinn, 1985). In this conditioning paradigm, there are thus two salient signals that must converge in the brain - the neural representations of the conditioned stimulus (the odour, CS) and the unconditioned stimulus (the electric shock/sugar, US). Given the mapping of the olfactory pathway described in section 1.3, we know that the KCs are responsive to odours, and from studies of the dopaminergic system, we know that the PPL1 cluster of DANs is activated by electric shock and the PAM cluster is activated by sugar (e.g., Burke et al., 2012; Cohn et al., 2015). Thus, the convergence of these two neural populations - KCs and DANs - presents a site at which CS and US become associated such that activation of the CS pathway leads to the conditioned response (CR) in the absence of US signalling.

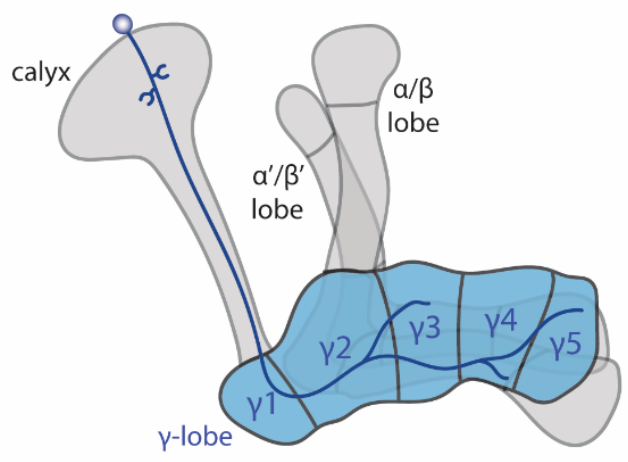

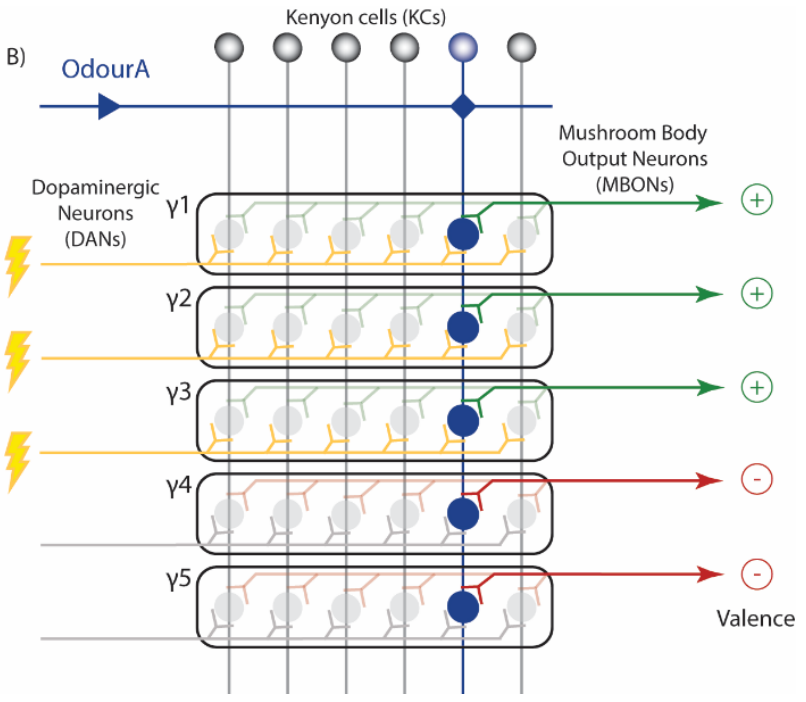


Figure 1.3.: Structural and functional compartmentalisation of the Mushroom Body. (A) The three lobes of the mushroom body (MB) formed of Kenyon cell (KC) axons $-\alpha / \beta, \alpha^{\prime} / \beta^{\prime}$, and $\gamma-$ are further compartmentalised. The $\gamma$ lobe is shown as an example, divided into five compartments $-\gamma 1-5$. (B) Each of the compartments of the $\gamma$ lobe have different input and output neurons (Dopaminergic neurons (DANs), left, and Mushroom Body Output Neurons (MBONs), right). Broadly, the DANs that have inputs to the 1 1-3 compartments belong to the electric shock-responsive PPL1 cluster of DANs, while the $\gamma 4$ and 5 compartments receive input from DANs of the PAM cluster that are responsive to positive reinforcement (such as sugar). The MBONs that receive input in the $11-3$ compartments encode positive valence, while those in $\gamma 4$ and 5 encode negative valence. This basic, 3-part circuit structure - KCs, MBONs, and DANs - and their interconnections are posited to represent the scaffold upon which associative memory traces are built.

Current models postulate the rutabaga-encoded type 1 calcium/calmodulin-dependent adenylate cyclase (Rut-AC) in KCs as the molecular coincidence detection site (Gervasi et al., 2010). The calcium influx that occurs in KCs due to odour stimulation, temporally paired with activation of G-protein coupled receptors that bind dopamine (released onto the MB by reinforcer-signalling DANs) leads to activation of Rut-AC resulting in increases in local cAMP and PKA activation (Gervasi et al., 2010). The necessity of this molecular pathway in associative conditioning is exemplified by the requirement for vital players in this cascade in successful odour-sugar or odour-electric shock learning - i.e., Rut-AC (Zars et al. 2000b; Livingstone, Sziber, and Quinn 1984), G-protein coupled dopamine receptors (Kim et al., 2007), PKA (Drain et al., 1991), and the cAMP-specific phosphodiesterase encoded by the dunce gene (Byers et al., 1981; Dudai et al., 1976). PKA has many downstream phosphorylation targets that can modulate synaptic plasticity. Most notably, PKA phosphorylates the transcription factor cAMP response element-binding protein (CREB) which then binds to the cAMP response element (CRE) and leads to increased transcription of downstream target genes required for LTM (Yin et al., 1994, 1995). In the context of STM, PKA also phosphorylates synapsin, which mediates vesicle release (Hilfiker et al., 1999; Michels et al., 2005) and can therefore influence the dynamics of neurotransmitter release to postsynaptic partners.

Functional imaging techniques that allow for the visualisation of calcium, CAMP, and PKA dynamics have helped to further our understanding of how this pathway is involved in the mediation of learning and memory formation. In fact, learning-dependent changes in odour-evoked intracellular calcium concentration has been demonstrated in each of the MB lobes (Akalal et al., 2010; Wang et al., 2008; $\mathrm{Yu}$ et al., 2006) using genetically-encoded calcium indicators. Likewise, changes in KC intracellular cAMP and PKA both also seem to accompany learning and memory formation in a dopamine- and RutAC-dependent manner (Boto et al., 2014; Gervasi et al., 2010; Louis et al., 2018). Thus, it appears clear 
that the molecular detection of CS and US coincidence in the MB KCs, and the subsequent cellular signalling pathways, are fundamental to associative learning.

In order for this association to result in a change of behavioural programme, there must be a readout of these molecular changes that underlies a shift in the valence of the CS. The MBONs, which receive broad input from the $\mathrm{KC}$ population, have arisen as the strongest candidate for this MB readout role. Indeed, multiple studies have demonstrated the importance of neurotransmitter release from the KCs in learning and memory tasks (Dubnau et al., 2001; McGuire et al., 2001; Schwaerzel et al., 2002). As such, the predominant models of the last two decades have designated the KC-to-MBON synapses as the target of learning-induced synaptic plasticity, triggered by the coincidence detection mechanism outlined above (see Heisenberg, 2003).

Barnstedt et al. showed that these synapses are cholinergic, with the expression of the vesicular acetylcholine transporter (VAChT) in the KCs and acetylcholine receptors on MBONs being required for odour-evoked activity in the MBONs (Barnstedt et al., 2016). During associative olfactory conditioning, specific populations of DANs are activated by a US and release dopamine onto specific compartments along the MB lobes - such as in the case of odour-electric shock conditioning, the electric shock stimulus activates the PPL1 cluster of DANs that then feed this information to the vertical lobes and heel region of the MB (Cohn et al., 2015; Riemensperger et al., 2005). Functional imaging and electrophysiological studies of the MBONs have shown that this input (paired with odour presentation) leads to a reduction in the subsequent odour-evoked activity of the MBONs that innervate the same MB compartments - i.e., the MBONs that innervate the $\gamma 1$ (Hige, Aso, Modi, et al., 2015; Perisse et al., 2016) and $ү 2$ (Berry et al., 2018) compartments. These two MBONs have been shown to mediate approach behaviour when optogenetically activated (Aso et al., 2014b). This represents a model circuit structure for the formation and readout of associative olfactory memories. Similar mechanisms seem to be at play in the avoidance coding MBONs, in which odour-evoked activity is increased as a result of aversive odour conditioning (Felsenberg et al., 2018; Perisse et al., 2016). 


\subsection{Overview of current work}

The work presented here aims to further dissect how odours are represented within the MB network. In sections 4-7, we focus on how an odour is encoded by the KCs and the MBONs before and after that odour is learned to be predictive of punishment, with the aim of uncovering the principles that underlie learning-induced synaptic plasticity within the KC-MBON circuit. This is done using specifically tailored in vivo calcium imaging protocols described in detail in sections 1 and 3.

In section 8, we look at odour coding in the context of innate rather than learned valence by probing the odour response characteristics of an MBON type that is not involved in learning and memory formation. Data presented therein points toward a role in controlling ethologically relevant behaviours that presents an interesting avenue for future work. 


\section{Materials and Methods}

\subsection{Materials}

\subsubsection{Drosophila melanogaster strains}

\begin{tabular}{|c|c|c|}
\hline Genotype & Source & Reference \\
\hline $\begin{array}{l}\text { w[1118]; R13F04-GAL4.DBD; R93D10- } \\
\text { p65.AD } \\
\text { (MB112C) }\end{array}$ & $\begin{array}{l}\text { G.M.Rubin, Howard Hughes } \\
\text { Medical Institute, Janelia } \\
\text { Research Campus; } \\
\text { Bloomington Drosophila Stock } \\
\text { Centre \#68263 }\end{array}$ & Aso et al., 2014a \\
\hline $\begin{array}{l}\text { w[1118]; R19F09-GAL4.DBD; R25D01- } \\
\text { p65.AD } \\
\text { (MB077C) }\end{array}$ & $\begin{array}{l}\text { G.M.Rubin, Howard Hughes } \\
\text { Medical Institute, Janelia } \\
\text { Research Campus; } \\
\text { Bloomington Drosophila Stock } \\
\text { Centre \#68284 }\end{array}$ & Aso et al., 2014a \\
\hline $\begin{array}{l}\text { w[1118]; R94B10-GAL4.DBD; R52G04- } \\
\text { p65.AD } \\
\text { (MB083C) }\end{array}$ & $\begin{array}{l}\text { G.M.Rubin, Howard Hughes } \\
\text { Medical Institute, Janelia } \\
\text { Research Campus; } \\
\text { Bloomington Drosophila Stock } \\
\text { Centre \#68287 }\end{array}$ & Aso et al., 2014a \\
\hline $\begin{array}{l}\text { w[1118]; R53C03-p65.AD; R24E12- } \\
\text { GAL4.DBD/TM6B, Tb[1] } \\
\text { (MB298B) }\end{array}$ & $\begin{array}{l}\text { G.M.Rubin, Howard Hughes } \\
\text { Medical Institute, Janelia } \\
\text { Research Campus; } \\
\text { Bloomington Drosophila Stock } \\
\text { Centre \#68309 }\end{array}$ & Aso et al., 2014a \\
\hline $\begin{array}{l}\text { w[1118]; R15B01-p65.AD; R27G01- } \\
\text { GAL4.DBD } \\
\text { (MB210B) }\end{array}$ & $\begin{array}{l}\text { G.M.Rubin, Howard Hughes } \\
\text { Medical Institute, Janelia } \\
\text { Research Campus; } \\
\text { Bloomington Drosophila Stock } \\
\text { Centre \#68272 }\end{array}$ & Aso et al., 2014a \\
\hline
\end{tabular}




\begin{tabular}{|c|c|c|}
\hline $\begin{array}{l}\text { w[1118]; R64F07-p65.AD; R64F07- } \\
\text { GAL4.DBD } \\
\text { (MB622B) }\end{array}$ & $\begin{array}{l}\text { G.M.Rubin, Howard Hughes } \\
\text { Medical Institute, Janelia } \\
\text { Research Campus }\end{array}$ & Aso et al., 2014a \\
\hline $\begin{array}{l}\text { w[1118]; R64F07-p65.AD; R57C10- } \\
\text { GAL4.DBD } \\
\text { (MB242A) }\end{array}$ & $\begin{array}{l}\text { G.M.Rubin, Howard Hughes } \\
\text { Medical Institute, Janelia } \\
\text { Research Campus; } \\
\text { Bloomington Drosophila Stock } \\
\text { Centre \#68307 }\end{array}$ & Aso et al., 2014a \\
\hline w[1118]; UAS-homer-GCaMP3.0/CyO & Fiala Lab stock collection & Pech et al., 2015 \\
\hline w[1118];20XUAS-IVS-GCaMP6f & $\begin{array}{l}\text { D.S. Kim, Howard Hughes } \\
\text { Medical Institute, Janelia } \\
\text { Research Campus; } \\
\text { Bloomington Drosophila Stock } \\
\text { Centre \#52869 }\end{array}$ & Chen et al., 2013 \\
\hline w[*];UAS-mCD8::GFP, UAS-n-syb::GFP & Fiala Lab stock collection & $\begin{array}{l}\text { Riemensperger et } \\
\text { al., } 2013\end{array}$ \\
\hline
\end{tabular}

\subsubsection{Standard fly food medium}

\begin{tabular}{|l|l|l|}
\hline Component & Quantity (per 20l) & Source \\
\hline Agar & $205 \mathrm{~g}$ & Gourvita GmbH \\
\hline Soy flour & $200 \mathrm{~g}$ & $\begin{array}{l}\text { Pflanzensaftwerk GmbH \& } \\
\text { Co. KG }\end{array}$ \\
\hline Yeast & $360 \mathrm{~g}$ & Gourvita GmbH \\
\hline Cornmeal & $1600 \mathrm{~g}$ & Zieller \& Co. GmbH \\
Syrup & $440 \mathrm{~g}$ & Obermühle Rosdorf \\
\hline $\begin{array}{l}\text { Malt } \\
\text { Sropionic acid }\end{array}$ & $126 \mathrm{ml}$ & $\begin{array}{l}\text { MeisterMarken - Ulmer } \\
\text { Spatz }\end{array}$ \\
\hline Nipagin & $30 \mathrm{~g}$ & Carl Roth GmbH + Co. KG \\
\hline Ethanol & $140 \mathrm{ml}$ & Sigma-Aldrich Chemie GmbH \\
\hline
\end{tabular}




\subsubsection{Chemicals}

\begin{tabular}{|l|l|}
\hline Chemical name & Source (Cat. No.) \\
\hline Triton X-100 & Carl Roth GmbH + Co. KG (3051) \\
\hline Bovine Serum Albumin (BSA) & Carl Roth GmbH + Co. KG (0163) \\
\hline Normal goat serum (NGS) & Invitrogen (31873) \\
\hline Paraformaldehyde & Carl Roth GmbH + Co. KG (0335) \\
\hline Mineral Oil & Sigma-Aldrich (M8410) \\
\hline 4-Methycyclohexanol & Sigma-Aldrich (153095) \\
\hline 3-Octanol & Sigma-Aldrich (218405) \\
\hline 1-Octen-3-Ol & Sigma-Aldrich (297887) \\
\hline Benzaldehyde & Sigma-Aldrich (12010) \\
\hline Cis-Vaccinyl Acetate & Cayman Chemical Company (10010101) \\
\hline VectaShield (mounting medium) & Vector Laboratories (H-1000-10) \\
\hline
\end{tabular}

\subsubsection{Solutions}

\begin{tabular}{|c|c|}
\hline Solution name & Composition \\
\hline Ringer's Solution (adult) & $\begin{array}{l}5 \mathrm{mM} \mathrm{KCl}, 130 \mathrm{mM} \mathrm{NaCl}, 2 \mathrm{mM} \mathrm{MgCl} 2 * 2 \mathrm{H}_{2} \mathrm{O}, 2 \mathrm{mM} \\
\mathrm{CaCl}_{2}, 5 \mathrm{mM} \text { Hepes, } 36 \mathrm{mM} \text { sucrose }(\mathrm{pH} 7.3 \text {, adjusted } \\
\text { with } \mathrm{NaOH} \text { and } \mathrm{HCl})\end{array}$ \\
\hline Ringer's Solution (larva) & $\begin{array}{l}2 \mathrm{mM} \mathrm{KCl}, 128 \mathrm{mM} \mathrm{NaCl}, 4 \mathrm{mM} \mathrm{MgCl}{ }_{2} * 2 \mathrm{H}_{2} \mathrm{O}, 18 \mathrm{mM} \\
\mathrm{CaCl}_{2}, 5 \mathrm{mM} \text { Hepes, } 36 \mathrm{mM} \text { sucrose }(\mathrm{pH} 7.1 \text {, adjusted } \\
\text { with } \mathrm{NaOH} \text { and } \mathrm{HCl})\end{array}$ \\
\hline Phosphate Buffered Saline (PBS) & 15 mM NaH2PO4, 100 mM NaCl, 85 mM Na2HPO4 \\
\hline PBS + Triton X-100 (PBS-T) & $0.6 \%$ Triton $\mathrm{X}-100$ in PBS \\
\hline Blocking Solution & $2 \%$ BSA in PBS-T \\
\hline Fixing solution (paraformaldehyde, PFA) & $4 \%$ PFA, $0.1 \% \mathrm{NaOH}$, in PBS \\
\hline
\end{tabular}




\subsubsection{Antibodies}

\begin{tabular}{|l|l|l|}
\hline Antibody & Source (Cat. No.) & Concentration used \\
\hline Mouse anti-discs large (DLG) & $\begin{array}{l}\text { Developmental Studies } \\
\text { Hybridoma Bank (4F3) }\end{array}$ & $1: 200$ \\
\hline Rabbit anti-GFP & Invitrogen (A6455) & $1: 2000$ \\
\hline Mouse anti-GFP-20 & Sigma-Aldrich (G6539) & $1: 200$ \\
\hline Mouse anti-ChAT4B1 & DSHB (AB_528122) & $1: 150$ \\
\hline Mouse anti-1D4 anti-Fascilin II & DSHB (AB_528235) & $1: 50$ \\
\hline $\begin{array}{l}\text { Mouse anti-IgG (AlexaFluor } \\
\text { 633) }\end{array}$ & Invitrogen (A21050) & $1: 300$ \\
\hline $\begin{array}{l}\text { Rabbit anti-IgG (AlexaFluor } \\
\text { 488) }\end{array}$ & Invitrogen (A11034) & $1: 300$ \\
\hline
\end{tabular}

\subsubsection{Consumables}

\begin{tabular}{|l|l|}
\hline Item name & Source (Cat. No.) \\
\hline Microscope slides & Carl Roth GmbH + Co. KG (0656.1) \\
\hline Clear adhesive tape & Tesa SE (56110) \\
\hline Blue light curing glue & Kent Express Limited (953683) \\
\hline Blue light lamp & $\begin{array}{l}\text { mectron Deutschland GmbH } \\
\text { (05100083-001) }\end{array}$ \\
\hline Forceps & Fine Science Tools GmbH (11412-11) \\
\hline Surgical scalpel blade & Swann-Morton (0303) \\
\hline Surgical scalpel blade holder & Swann-Morton (Cat. No. 0907) \\
\hline Insect pins & Fine Science Tools GmbH (26002-10) \\
\hline Concave-convex jaws & Fine Science Tools GmbH (10053-09) \\
\hline Microknife & Fine Science Tools GmbH (10315-12) \\
\hline
\end{tabular}


2.1.7. Microscope hardware and software

\begin{tabular}{|c|c|c|}
\hline Use & Item name & Source \\
\hline \multirow[t]{5}{*}{ Two-photon microscopy } & LSM 7MP & Carl Zeiss AG \\
\hline & Ti-Sapphire laser & Coherent Inc. \\
\hline & Dichroic mirror & Carl Zeiss AG \\
\hline & $\begin{array}{l}\text { Plan-Apochromat } 20 x \text { water } \\
\text { immersion objective }(N A=1)\end{array}$ & Carl Zeiss AG \\
\hline & Zen 2011 SP4 (software) & Carl Zeiss AG \\
\hline \multirow[t]{6}{*}{ Confocal microscopy } & $\begin{array}{l}\text { SP8 confocal laser scanning } \\
\text { microscope }\end{array}$ & Leica Microsystems GmbH \\
\hline & $\begin{array}{l}\text { APO 20x glycerol/water objective } \\
\text { (NA }=0.75 \text { ) }\end{array}$ & Leica Microsystems GmbH \\
\hline & Argon-laser (488nm) & Leica Microsystems GmbH \\
\hline & DPSS-laser (561nm) & Leica Microsystems GmbH \\
\hline & HeNe-laser (633nm) & Leica Microsystems GmbH \\
\hline & $\begin{array}{l}\text { Leica Application Suite X (LASX; } \\
\text { software) }\end{array}$ & Leica Microsystems GmbH \\
\hline
\end{tabular}

2.1.8. Other software

\begin{tabular}{|l|l|}
\hline Software & Source \\
\hline ImageJ/FIJI & National Institutes of Health (NIH) \\
\hline Labview & National Instruments \\
\hline OriginPro 2020 & OriginLab Corp. \\
\hline Microsoft Office Excel & Microsoft Crop. \\
\hline Matlab & Mathworks \\
\hline
\end{tabular}




\subsection{Methods}

\subsubsection{Fly husbandry and maintenance}

Flies were raised on standard cornmeal food medium in incubators maintained at $25^{\circ} \mathrm{C}$ and $60 \%$ relative humidity with a $12 \mathrm{hr} / 12 \mathrm{hr}$ light/dark cycle.

In experiments investigating the influence of the olfactory rearing environment (shown in Figures 8.4. and 8.5.), food vials also contained a $0.2 \mathrm{ml} \mathrm{PCR} \mathrm{tube} \mathrm{containing} \mathrm{a} \mathrm{specific} \mathrm{odorant} \mathrm{-} \mathrm{either} \mathrm{MCH} \mathrm{or} \mathrm{3-}$ Octanol, both diluted to a concentration of 1:50 in mineral oil. Each PCR tube contained $100 \mu$ l of the diluted odorant and was embedded into the fly food with the lid facing upwards and perforated with a fine needle to allow odour vapour to be released into the vial. Each group (raised with $\mathrm{MCH}$, raised with 3-Octanol, or raised with only fly food) was kept in an isolated box, and in a well-ventilated incubator to avoid any cross-exposure to other odours. Flies were moved to fresh vials that contained a fresh odour tube every 2-3 days.

For experiments in which flies were starved beforehand (shown in Figures 8.6. and 8.7.), aged flies of the appropriate genotype were moved from standard food vials to empty plastic vials containing only moistened tissue paper 20-24 hours before the experiment. This ensured flies were sufficiently starved before the experiment, without depriving them of water.

\subsubsection{Fly preparation for in vivo calcium imaging}

Methods for preparation and imaging of flies are detailed in sections 3 and 5 (see also, Hancock et al., $2019,2020)$. This preparation procedure was used in all calcium imaging experiments.

Single flies were immobilized by placing them in an empty plastic vial on ice for approximately 5 minutes. Unless stated otherwise, female flies were used throughout. The fly was then moved to a custom-built chamber (see section 4, Figure 1) using fine forceps. The fly was fixed in place using clear adhesive tape. In the case of experiments concerning aversive olfactory associative conditioning, the fly chamber included two electrical wires, on top of which the fly thorax was placed, to facilitate the delivery of punishing electric shocks. The head of the fly was in all cases stabilised on a small platform such that the adhesive tape exerted light pressure on the dorsal surface of the head when applied.

To access the head of the fly, a small window was cut in the adhesive tape using a scalpel blade. This window allowed for the placement of blue light-curing glue around the head. When set using a blue light-emitting lamp, the glue limits movement of the head and body that could be disruptive during functional imaging experiments. 2-3 drops of room temperature Ringer's solution were then placed 
on top of the cuticle, and an incision made along the posterior of the head. Two more incisions were then made, perpendicular to the first and running just inside the eyes of the fly. Fine forceps were then used to rip off the cuticle to expose the inside of the head and to remove excess tissue on top of the brain. The fly, in the chamber, was then transferred to a microscope equipped for two-photon microscopy for imaging.

\subsubsection{Two-photon microscopy}

Two-photon excitation was used to visualise odour-evoked changes in intracellular calcium concentrations in neurons of interest. In all cases, a version of the genetically encoded calcium indicator GCaMP was used (in sections 3 and 4, homer-GCaMP3 was used (Pech et al., 2015); in sections 5 and 6, GCaMP3 was used (Tian et al., 2009); in section 7, GCaMP6f was used (Chen et al., 2013)). In all cases, an excitation wavelength of 920nm was used. Emitted light was filtered by a customised filter set composed of a $605 \mathrm{~nm}$ beam splitter and a bandpass filter that selectively filters GFP emission between 500-550nm.

Microscope software (Zen 2011 SP4) was used to set scanning parameters and to control image acquisition. A framerate of $4 \mathrm{~Hz}$ and a frame size of $512 \times 512$ pixels was used throughout. To synchronise image acquisition with delivery of odours, a custom-written programme run by LabView software was used in conjunction with the Visual Macro Editor function of the Zen software. With this programme, it was possible to control the timing of odour delivery such that odour onset and offset can be documented for later analysis steps.

For aversive associative conditioning experiments (sections 3-6), flies were exposed to three different odours: $\mathrm{MCH}, 3-O c t a n o l$, and 1-Octen-3-ol at concentrations of 1:750, 1:500, and 1:400, respectively in mineral oil. Flies were also presented with mineral oil alone. Odour presentations each lasted for $2.5 \mathrm{sec}$ and were separated from one another by approximately $40 \mathrm{sec}$. In experiments in which the dendritic compartments of $\gamma$-lobe MBON were imagined (sections 4 and 5), only a single plane encompassing the dendritic compartment of the neuron was visualised and was optimised for each fly such that the plane was as similar as possible between individual flies and to achieve a broad crosssection of the dendritic tree. 


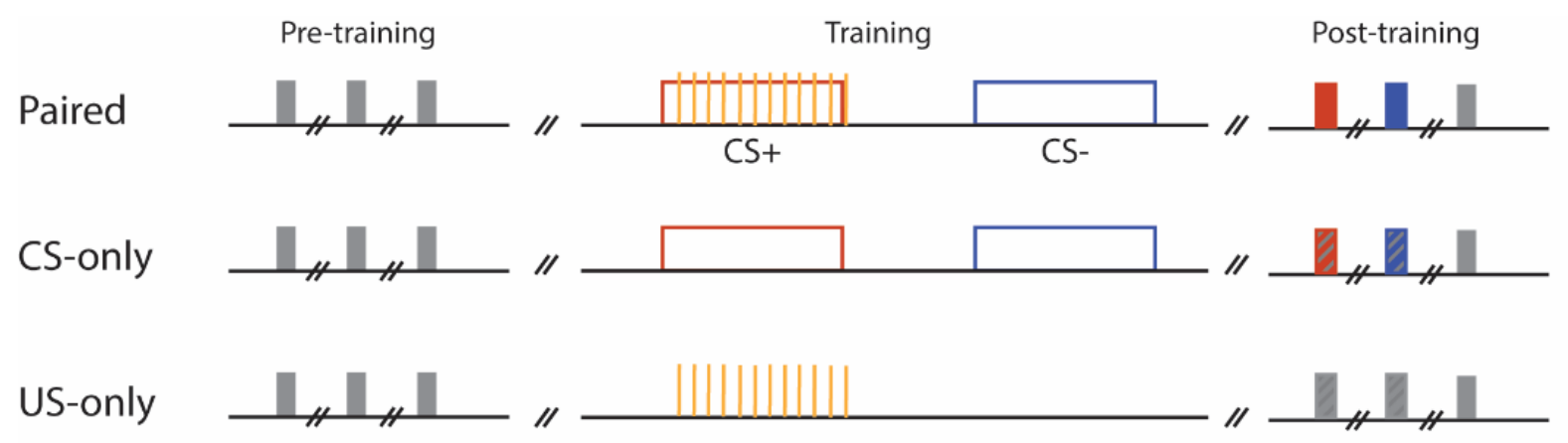

Figure 2.1.: Odour presentation and conditioning protocols for in vivo calcium imaging. All flies were presented with three odours in pre-training and post-training phases, in which odour-evoked changes in calcium were visualised and quantified. In the training phase, flies were subjected to either a classical conditioning protocol (paired) or a control protocol (CS-only or US-only) that does not lead to associative learning.

Aversive olfactory conditioning was conducted with the use of a specialised LabView protocol. This protocol additionally incorporates the presentation of an electric shock procedure, such that the fly is exposed to a classical, aversive Pavlovian conditioning paradigm (Figure 2.1.). Therein, flies are first presented with an odour (becoming the Conditioned Stimulus +, or $\mathrm{CS}+$ ) for 60 seconds, during which the flies also receive a pulsating electric shock ( $12 \times 90 \mathrm{~V}$ shocks) to the thorax (the Unconditioned Stimulus, or US). This is followed by a 60 second break. After this break, the flies are then exposed to a second odour without electric shock (becoming the Conditioned Stimulus-, or CS-) for 60 seconds. Within each experimental group, flies were trained reciprocally with either $\mathrm{MCH}$ as the $\mathrm{CS}+$ and 3Octanol as the CS-, or vice versa. Flies that were subjected to this protocol are described as the "paired" group here, denoting the pairing of odour and electric shock that classically leads to learned avoidance of the CS+ odour.

After the training protocol, flies were then exposed to the same odour presentation protocol as before training (i.e., flies were presented with the same three odorants and the responses monitored). Thereby, it was possible to examine the odour-evoked calcium transients elicited by odours that have no strong meaning to the fly and odours that have gained a valence through the process of electric shock-odour pairing.

Two control groups were used to control for the effects of mere exposure to the two stimuli described - odour exposure and electric shock exposure. The "CS-Only" group received the exact same protocol as the paired group, with the exception of the electric shock delivery. The "US-Only" group received the exact same protocol as the paired group, with the exception of the extended odour delivery. 
For MB-CP1 odour tuning experiments (section 7), flies were exposed to six different odorants. $\mathrm{MCH}$, 3-Octanol, benzaldehyde (BA), and cis-Vaccinyl acetate ( $\mathrm{CVA}$ ) were diluted in mineral oil at concentrations of 1:750,1:500,1:200, and 1:1000, respectively. Apple vinegar was diluted in water to a concentration of 1:100. Finally, approximately $2 \mathrm{~g}$ of standard fly food medium was homogenised in $2 \mathrm{ml}$ of tap water. Measurements were conducted at the dendritic shaft region (see Figure 2.2.). The
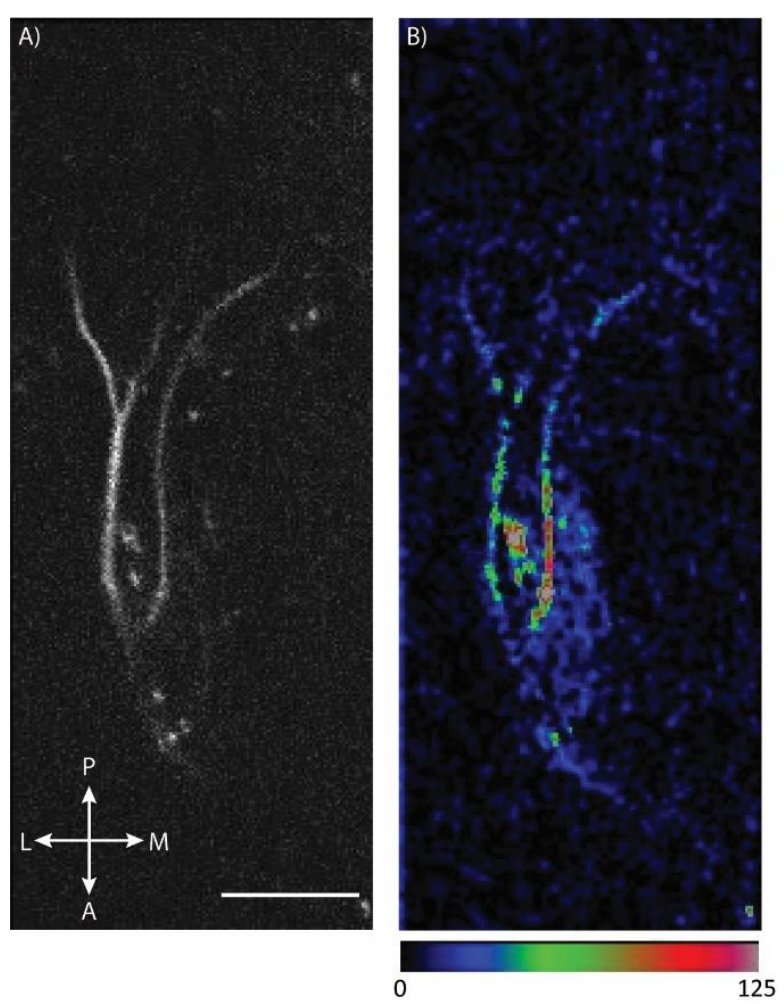
imaging plane was matched as best as possible between individuals, although slight variations in the tilt of the head meant this was not exact in all cases. The odour presentation protocol was such that each odour was presented twice in a randomized order in each fly.

Figure 2.2.: Example MB-CP1 imaging plane. (A) Grayscale single frame image from an example imaging plane as used in the in vivo measurement of odour responses in MB-CP1, at the level of the dendritic shaft. Scale bar $=15 \mu \mathrm{m}$. (B) False colour coded depiction of the same imaging frame as $(A)$ during stimulation with apple vinegar.

\subsubsection{Image analysis - calcium imaging}

All image analysis was conducted using ImageJ (NIH). All functional imaging sequences were registered using the TurboReg plugin (Thévenaz et al., 1998) to remove small movement artefacts. A region of interest (ROI) was then placed around the structure of interest. In the case of $\gamma$-MBON measurements (sections 3 and 4), the ROI was placed around the dendritic tree of the neuron. Due to the sometimesweak baseline signal of the homer-GCaMP, this was often facilitated by using an average projection over time to make borders more easily visible. In the case of the MB-CP1 measurements (section 7), a single rectangular ROI was placed to best encompass the dendritic shaft region of the neuron(s). Fluorescence values were extracted from the raw images using ImageJ and were subsequently used to calculate the normalised change in fluorescence over time $-\Delta F / F_{0}$. The $F_{0}$ value was calculated as the mean fluorescence over the 2-4 seconds immediately preceding odour presentation. This was then subtracted from each value over time to generate a $\Delta \mathrm{F}$ series. The $\Delta \mathrm{F}$ was then divided by the $\mathrm{F}_{0}$ to 
normalise each trace to the baseline fluorescence. These calculations and all following tabulations were carried out using Excel and Origin Pro 2020.

\subsubsection{Immunohistochemistry - adult Drosophila brain}

This procedure was used to generate anatomical images shown in Figure 5.1., A and Figure 7.1., A and B.

Flies were immobilised by placing them in an empty plastic vial on ice. A single fly was then removed from the vial using fine forceps and placed ventral side up in a dissection dish filled with dyed silicone mixture. The fly was fixed in place using two insect pins - one through the thorax and another through the distal abdomen. 3-4 drops of ice-cold Ringer's solution was pipetted onto the fly, such that it was entirely submerged. Using fine forceps, the proboscis was removed to better facilitate the further removal of the head capsule. Excess tissue was then carefully removed, and the brain detached from the ventral nerve cord. The brain was then transferred to a glass dish containing a fixative solution containing $4 \%$ paraformaldehyde in PBS for $2 \mathrm{hr}$ at $4^{\circ} \mathrm{C}$ or $45 \mathrm{~min}$ at room temperature. The brains were then washed 3 times for 20 min each in PBS with $0.6 \%$ Triton-X (PBS-T) at room temperature. Brains were then transferred to a blocking solution, formed of PBS-T and $2 \%$ bovine serum albumin, and were incubated for 2 hours at room temperature. Then, brains were incubated overnight at $4^{\circ} \mathrm{C}$ in primary antibody mix, containing primary antibodies against proteins of interest diluted in blocking solution. For each antibody used in this study, the concentrations used can be found in the Materials. Brains were then washed, as previously, three times for 20 minutes each in PBS-T. They were then incubated overnight at $4^{\circ} \mathrm{C}$ in secondary antibody mix. All secondary antibodies were used at a concentration of 1:300 in blocking solution. After this incubation, brains were washed again as before and then mounted in VectaShield on microscope slides. Brains were placed in a drop (approximately $6 \mu \mathrm{l})$ of VectaShield held in the centre of two tape rings and covered with a glass cover slide. The cover slides were then fixed in place using clear nail polish. Slides were stored in darkness at $4^{\circ} \mathrm{C}$ until confocal scanning.

\subsubsection{Immunohistochemistry - larval Drosophila brain}

This procedure was used to generate anatomical images shown in Figure 7.1., C-F and was carried out by Maria Woitow.

Larvae were removed from food vials using a small spatula and placed in a glass dish containing larval Ringer's solution on ice, to immobilise. Individual larvae were then transferred to a dissection dish, as 
used above, using forceps. To access the brain, fine forceps were used to pull from the mouth hooks and the posterior of the larva simultaneously and in opposite directions. With the body wall then torn open, excess tissue was removed from the brain using fine forceps. The brain was then transferred to fixative solution (as above) for $60 \mathrm{~min}$ at room temperature. Brains were then washed once for $10 \mathrm{~min}$ and then twice for 30 min each in PBS-T solution (for larvae, 3\% Triton-X in PBS was used). Brains were then moved to a blocking solution containing $2 \%$ bovine serum albumin and $2 \%$ normal goat serum in PBS-T and were incubated for $90 \mathrm{~min}$ at room temperature. The brains were then incubated in a primary antibody mix for two days at $4^{\circ} \mathrm{C}$. After primary antibody incubation, brains were washed using PBS-T (twice for 10 minutes each, followed by three times for $30 \mathrm{~min}$ each). Then, brains were incubated in secondary antibody mix for one day at $4^{\circ} \mathrm{C}$ and then again washed with PBS-T (1x 5 min, $1 \times 10 \mathrm{~min}, 2 \times 3 \mathrm{~min}$ ) and additionally with PBS, 2x for $5 \mathrm{~min}$ each. Larval brains were then mounted in the same way as the adult brains, with the exception that only a single tape ring is used due to the smaller size of the brain. Slides were also stored in darkness at $4^{\circ} \mathrm{C}$ until confocal scanning.

\subsubsection{Confocal microscopy}

Immunostained and mounted brains were scanned using a Leica SP8 line scanning microscope equipped with either a $20 x$ or $63 x$ glycerol immersion lens. Scanning and image acquisition was controlled using Leica LSAX software. Excitation lasers of wavelengths $488 \mathrm{~nm}$ (Argon-laser), 561nm (DPSS-laser), and 633nm (HeNe-laser) were used, depending on the secondary antibodies used. Laser and detector settings were optimised for each brain to ensure high resolution and minimal sample bleaching. Image stacks were captured and saved as .lif files for later processing.

\subsubsection{Classical olfactory conditioning}

These procedures were used to generate data shown in Figure 7.3. and were carried out by Haiko Poppinga.

For appetitive conditioning assays, flies were starved for 24 hours before experiments. Groups of 50100 flies aged 3-5 days old were loaded into a training apparatus based on that used by Tully and Quinn (Tully \& Quinn, 1985). Flies were then left to acclimate for 3 minutes before starting conditioning steps. The flies were then moved into training tubes that were fitted with either an electrifiable copper wire lining around the inside or with slots into which filter paper soaked with a sucrose mix can be fitted on the inner walls. These tubes are used to deliver punishing or rewarding unconditioned stimuli (US), respectively. An odour (CS+) was then delivered via a controlled air flow 
through the tube. To induce aversive associative learning, this odour delivery was accompanied simultaneously with pulses of electric current through the copper wire such that flies in contact with it received electric shocks ( $12 \times 90 \mathrm{~V}$ pulses over one minute). To induce appetitive associative learning, odour delivery was paired with sucrose by inserting filter paper soaked in a $2 \mathrm{M}$ sucrose solution to the training tube. This pairing step lasted for one minute, after which time odour delivery and US delivery stopped and flies were exposed only to a clean air flow for one minute. After this break, flies were transferred to new tubes (in the opposite arm of the T-maze) and exposed to an odour (CS-) for one minute, without the US. In appetitive conditioning, CS- tubes contained filter paper soaked with water. Flies were then transferred to a holding position in the middle of the T-maze where they were kept for 3 minutes with no further stimulation. Flies were then released from this holding position and allowed to disperse between the arms of the T-maze - with the CS+ and CS- odours being delivered from opposite arms simultaneously. After two minutes, the arms were isolated, and the number of flies in each arm was counted. From these values, a learning index was calculated

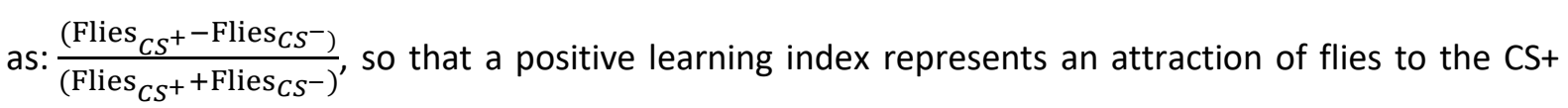
odour and a negative value represents an avoidance of the CS+ odour. In all conditioning experiments, odours were trained reciprocally such that each learning index is pooled from experiments in which the assignment of an odour as CS+ or CS- is balanced to reduce the chance of odour identity influencing learning scores.

\subsubsection{Statistics}

All calcium traces are displayed as mean $\Delta \mathrm{F} / \mathrm{F}_{0}$ values over time, averaged across animals of the same experimental group, with shaded areas showing standard error of the mean (SEM). In Figure 4.2., the number of responsive neurons measured was calculated as a percentage of total measured neurons that showed a $\Delta F / F_{0}$ value during the response period ( $2.5 \mathrm{sec}$ odour presentation plus $2.5 \mathrm{sec}$ after) that exceeded 3 times the pre-stimulation standard deviation. In Figures 4.3. and 4.4., box plots show the median and upper and lower quartiles and whiskers show the full data range. These data are extracted from the $\Delta F / F_{0}$ traces of individual flies by calculating the integrated area under the curve (AUC) during the response period. Tests for significant changes in pre-to-post AUC was done using the paired Wilcoxon signed ranks test. In Figure 7.3., bars represent mean learning indices across repetitions and error bars show SEM. Normality of data distribution was tested using the Shapiro-Wilk test. For normally distributed data, as in Figure 7.3., a one-way ANOVA was used to test for significant difference between groups followed by Bonferroni post hoc test. Scatter plots in Figures 7.4., 7.5., 7.6., and 7.7. show odour responses as AUC and mean (coloured dot). Bars show median and $95 \%$ 
confidence intervals $(\mathrm{Cl})$. Normality was again tested with the Shapiro-Wilk test. These data did not show a normal distribution. Therefore, tests for inter-group/inter-condition difference were conducted with a Kruskal-Wallis ANOVA, followed by Dunn's post-hoc test. 


\section{Visualisation of Learning-Induced Plasticity at the MBON Postsynapse}

\subsection{Introduction and aims}

In this section, one can find the publication, "In Vivo Optical Calcium Imaging of Learning-Induced Synaptic Plasticity in Drosophila melanogaster" in which we detail the primary experimental technique used throughout the studies presented in this thesis. Per the title, this paper documents the process of visualisation of calcium dynamics in the living fruit fly brain, with an emphasis on the use of this technique for the quantification of learning-induced changes in activity that arise due to a classical odour conditioning protocol.

The dissection of the neuronal circuits underlying complex behaviours such as learning is of great interest in the field of neurobiology. Therefore, by combining a long-established protocol for the induction of short-term olfactory associative learning with in vivo calcium imaging, we present a technique capable of bridging the gap between behaviour and patterns of activity in the brain. Furthermore, by demonstrating the use of a subcellularly localised genetically encoded calcium indicator (homer-GCaMP3 (Pech et al., 2015)), we describe a way in which one can further localise changes in calcium dynamics that occur throughout the learning process and thus provide a tool for more precise circuit dissection.

As an example of this visualisation, we used a well characterised mushroom body output neuron, MBON- $\gamma 1$ (also called MVP2), that has been shown to play a role in and be modulated by olfactory learning tasks (Hige, Aso, Modi, et al., 2015; Perisse et al., 2016). By expressing the homer-GCaMP transgene in this neuron and exposing flies to odour stimuli, we were able to observe odour-evoked changes in intracellular calcium specifically at the postsynapse. By then comparing these changes in calcium before and after pairing of an odour with aversive electric shock stimuli, we quantified how the odour-driven input to this neuron is modulated by the assignment of that odour as predictive of a strong punishment. 
3.2. Manuscript - In vivo Calcium Imaging of Learning-Induced Synaptic Plasticity in Drosophila melanogaster

Journal of Visualized Experiments, October 2019

Authors: Clare E. Hancock, Florian Bilz, André Fiala

Contribution:

Wrote the manuscript together with supervisor Prof. Dr. André Fiala, conducted the experiments described, and performed the filmed demonstration. 


\title{
Video Article \\ In Vivo Optical Calcium Imaging of Learning-Induced Synaptic Plasticity in Drosophila melanogaster
}

\author{
Clare E. Hancock ${ }^{1}$, Florian Bilz ${ }^{1}$, André Fiala ${ }^{1}$ \\ ${ }^{1}$ Department of Molecular Neurobiology of Behavior, University of Göttingen
}

Correspondence to: André Fiala at afiala@gwdg.de

URL: https://www.jove.com/video/60288

DOI: doi:10.3791/60288

Keywords: Neuroscience, Issue 152, Drosophila melanogaster, mushroom body, synaptic plasticity, learning and memory, olfactory associative learning, classical conditioning, optical calcium imaging, two-photon microscopy, memory engram

Date Published: 10/8/2019

Citation: Hancock, C.E., Bilz, F., Fiala, A. In Vivo Optical Calcium Imaging of Learning-Induced Synaptic Plasticity in Drosophila melanogaster. J Vis. Exp. (152), e60288, doi:10.3791/60288 (2019)

\section{Abstract}

Decades of research in many model organisms have led to the current concept of synaptic plasticity underlying learning and memory formation Learning-induced changes in synaptic transmission are often distributed across many neurons and levels of processing in the brain. Therefore, methods to visualize learning-dependent synaptic plasticity across neurons are needed. The fruit fly Drosophila melanogaster represents a particularly favorable model organism to study neuronal circuits underlying learning. The protocol presented here demonstrates a way in which the processes underlying the formation of associative olfactory memories, i.e., synaptic activity and their changes, can be monitored in vivo. Using the broad array of genetic tools available in Drosophila, it is possible to specifically express genetically encoded calcium indicators in determined cell populations and even single cells. By fixing a fly in place, and opening the head capsule, it is possible to visualize calcium dynamics in these cells whilst delivering olfactory stimuli. Additionally, we demonstrate a set-up in which the fly can be subjected, simultaneously, to electric shocks to the body. This provides a system in which flies can undergo classical olfactory conditioning - whereby a previously naïve odor is learned to be associated with electric shock punishment - at the same time as the representation of this odor (and other untrained odors) is observed in the brain via two-photon microscopy. Our lab has previously reported the generation of synaptically localized calcium sensors, which enables one to confine the fluorescent calcium signals to pre- or postsynaptic compartments. Two-photon microscopy provides a way to spatially resolve fine structures. We exemplify this by focusing on neurons integrating information from the mushroom body, a higher-order center of the insect brain. Overall, this protocol provides a method to examine the synaptic connections between neurons whose activity is modulated as a result of olfactory learning.

\section{Video Link}

The video component of this article can be found at https://www.jove.com/video/60288/

\section{Introduction}

Deciphering where and how the information is acquired in the brain through learning and subsequently stored as memory constitutes one of the most challenging tasks in neuroscience. Neuroscientific research has led to the concept of a change in synaptic transmission as the neuronal substrate that underlies learning and memory formation ${ }^{2,3}$. It is hypothesized that, during learning, synaptic connections between neuronal ensembles that are active during the perception of a stimulus become modified such that their combined activity pattern can be retrieved during memory recall, thereby instructing future behavioral action ${ }^{4}$. These "engram cells" and their synapses are often distributed across brain regions and levels of processing, which makes it difficult to assign observed changes in synaptic transmission to the learning of a task or a stimulus. To localize and visualize those synaptic changes that are causally linked to a specific learning task one needs an appropriate model system that allows for precisely confining those synapses.

For such an endeavor, Drosophila melanogaster is particularly suitable because it combines relative brain simplicity, behavioral richness, and experimental accessibility. Among the well-established model organisms, Drosophila is situated between the nematode $C$. elegans and genetically tractable mammals like mice in terms of neuronal complexity. The stereotypic number of neurons $(\sim 300)$ and limited behavioral repertoire is observed in $C$. elegans. Mammals, on the other hand, have millions of neurons and staggering behavioral complexity. The brain of the fruit fly is, with its $\sim 100,000$, neurons significantly smaller than the brains of most vertebrates, and many of the neurons are individually identifiable ${ }^{5}$. Yet, Drosophila demonstrate a broad spectrum of complex behaviors, including an ability to exhibit robust associative olfactory learning and memory formation, first described over 40 years ago ${ }^{6}$. In the course of this classical conditioning procedure, groups of flies are subjected to an odor as the conditioned stimulus $\left(\mathrm{CS}^{+}\right)$while they receive a punishing electric shock as the unconditioned stimulus (US). A second odor (CS) is then presented without any punishment. Thereby, the animals learn to avoid the odor associated with the punishment, which can be tested in a subsequent choice situation between the two odors, $\mathrm{CS}^{+}$and $\mathrm{CS}^{-}$. Work on dissecting the neuronal substrate underlying this behavior in Drosophila has identified the mushroom bodies (MB) as the primary site of the "engram" $7,8,9,10$ and, therefore, the circuitry of this brain region was and is the subject of intense research in order to uncover the logic by which a memory engram is acquired and stored (recently reviewed in ${ }^{11,12}$ ). 
The Drosophila MB consists of $\sim 2,000$ intrinsic neurons (Kenyon cells) per hemisphere, organized in parallel axonal projections ${ }^{13}$. Axons of olfactory projection neurons are extended to the lateral protocerebra and to the MB calyces, the main dendritic input site of the MB and receive olfactory input from antennal lobes. The long, parallel axons bundle of Kenyon cells constitute the peduncle and the lobes. Most Kenyon cells bifurcate forming horizontal $\beta / \beta^{\prime}$-lobes by extending one collateral towards the midline of the brain, and the vertical $\alpha / \alpha^{\prime}-$ lobes by extending second collateral projecting in the dorsal-anterior direction. The other group of Kenyon cells forms the horizontal $y$-lobes ${ }^{13}$ of the MB where the learning process and subsequent short-term memory formation could be localized ${ }^{16}$. The MB lobes receive afferent input and provide efferent output, both of which are typically restricted to distinct compartmental sub-regions along the Kenyon cell axons ${ }^{14,15,16}$. In particular, afferent dopaminergic MB input neurons have been shown to mediate value-based, e.g., punitive, reinforcing effects in associative olfactory learning ${ }^{15,17}$ Stereotypic and individually identifiable efferent MB output neurons from the mushroom body lobes integrate information across large numbers of Kenyon cells, target diverse brain areas and bear behavior-instructive appetitive or aversive information ${ }^{15}$. This neuronal architecture has led to a concept of the organization of the associative engram. Odors are relatively precisely encoded by sparsely activated ensembles of Kenyon cells. The coincident activity of these Kenyon cell ensembles and release of dopamine - evoked by punishing stimuli - modulates transmission from Kenyon cell presynapses onto MB output neurons such that the animals will subsequently avoid this particular smell ${ }^{10,12}$. We use this rather precisely defined and localized engram as a paradigmatic case to illustrate how these learning-dependent changes in synaptic activity can be determined and monitored.

The value of Drosophila as a model system relies strongly on the unmatched genetic toolbox that allows one to express transgenes for identifying, monitoring, and controlling single neurons within complex circuits ${ }^{18}$. The advent of techniques for neuronal activity monitoring such as calcium imaging, discussed here - have allowed for the determination of neuronal activity patterns in response to a specific stimulus. By combining specific Gal4-driven expression of genetically encoded calcium indicators (GECls) with olfactory stimulation, one can visualize the odor-evoked calcium dynamics of neurons of interest ${ }^{19}$. In this protocol, it is shown that by further coupling this technique with a classical conditioning paradigm, it is possible to examine these olfactory responses in the context of learning. Learning-induced plasticity can be further dissected using GECls that are not only localized to a single specific neuron, but also to specific subcompartments of a neuron. Pech et al. ${ }^{20}$ established a selection of tools that allow exactly this. By targeting GCaMP3 ${ }^{21}$ to either the pre- or postsynapse - via linkage to the vertebrate Synaptophysin or $d$ Homer, respectively ${ }^{20}$ - the differential modulation of these sites can be distinguished. This localization confers, in this context, an advantage over most GECls that are ubiquitously present throughout the cytosol - e.g., GCaMP ${ }^{22}, \mathrm{GCaMP}^{21}$, or GCaMP6 ${ }^{23}$ - because it means that pre- and postsynaptic transients can be distinguished from the overall integrated calcium influx that occurs as a result of neuron activation. This can provide clues about the location and types of plasticity that occur as a result of or that cause learning and memory formation. As an example, the protocol provided here shows the value of this tool in deciphering the modulation of MB output neurons during olfactory associative learning by targeting the expression of the calcium sensor to only the postsynapse. By monitoring, within an individual fly, odorevoked activity before and after olfactory conditioning a direct comparison can be drawn between a naïve odor response and a learned odor response. Whilst fixed in the same imaging chamber, flies are exposed to a selection of odors. Then, they receive an aversive associative conditioning protocol in which one of these odors is paired with electric shock (becoming the $\mathrm{CS}^{+}$) and another odor is presented without reinforcement (becoming the $\mathrm{CS}^{-}$). Finally, the flies are again exposed to the same odors as in the first step. Calcium dynamics are observed using two-photon microscopy.

Protocol

\section{Transgenic fruit flies, Drosophila melanogaster}

1. Cross female virgin and male flies (raised at $25^{\circ} \mathrm{C}$ in $60 \%$ relative humidity on a $12 \mathrm{~h}$ light/dark cycle) carrying the desired Gal4 and UAS constructs $^{25}$, respectively, to produce flies in which specific neurons of interest express a genetically encoded calcium indicator.

2. Age the female progeny of the above cross until they are in the range of 3-6 days post-eclosion. Female flies are preferable because of their slightly larger size.

\section{Preparation of the fruit fly for in vivo calcium imaging}

1. Select a single female fly and anesthetize on ice for no longer than $5 \mathrm{~min}$

2. Using fine forceps, place the fly in the imaging chamber (demonstrated in Figure 1c). Ensure the thorax and legs are in contact with the electrical wires in the bottom of the chamber and that the head lays flat. Fix the position of the fly using clear adhesive tape. NOTE: This protocol requires a custom-built chamber in which the flies are fixed, with the head capsule accessible for opening, and the flies able to receive both odor stimulations to the antennae and electric shocks to the thorax and legs (Figure 1).

3. Using a surgical scalpel blade fixed to the scalpel handle (see Table of Materials), cut a window in the tape around the head of the fly, leaving the antennae covered and only the anterior-most portion of the thorax exposed.

4. Surround the sides and back of the head with blue-light curing glue, carefully manipulated using an insect pin held by concave-convex jaws Set the glue using a blue light-emitting LED lamp.

5. Check that the glue is completely set and clear any residual unhardened glue from the dorsal surface of the fly head.

6. Apply a drop of Ringer's solution ${ }^{24}$ (see Table of Materials) to cover the exposed cuticle of the head.

7. Using a very fine-bladed stab knife, cut through the cuticle. For the most efficient removal of the cuticle, first cut across the posterior of the head using the ocelli as a starting point. Then, cut up each side, medial to the eyes, to form a flap of the cuticle that can be easily torn off using forceps.

8. Remove any excess cuticle that may block the brain region of interest.

9. Carefully clear the dorsal surface of any trachea using fine forceps, avoiding disruption of the brain tissue itself. Remove and refresh Ringer's solution $^{24}$ (see Table of Materials) as required to clear the area of tissue debris

10. Place the hypodermic odor delivery needle in position, approximately $1 \mathrm{~cm}$ from the head of the fly. Ensure there is nothing that could obstruct odor delivery to the antennae.

11. At the microscope, connect the imaging chamber to the odor-delivery system via the hypodermic odor delivery needle. 
12. To allow the fly to fully to recover from anesthesia and surgery, and to adapt to the air flow, implement a 10 min resting period at this stage.

\section{In vivo calcium imaging}

1. Use a multiphoton microscope equipped with an infrared laser and a water immersion objective (see Table of Materials), installed on a vibration-isolated table. For the visualization of GFP-based calcium indicators, tune the laser to an excitation wavelength of $920 \mathrm{~nm}$ and install a GFP band-pass filter.

2. Using the coarse $Z$ adjustment knob, scan through the $Z$ axis of the brain and locate the brain region of interest. Use the crop function to focus scanning on only this area to minimize scan time, and to rotate the scan view such that the anterior of the head is facing downwards.

3. Adjust the frame size to $512 \times 512 \mathrm{px}$, scan speed to $>4 \mathrm{~Hz}$, and scan region (in the $Y$ dimension) so that the neurons of interest are covered.

\section{Visualization of odor-evoked calcium transients through olfactory conditioning}

1. Use an odor delivery system ${ }^{19,26}$ that can deliver several odor stimuli in a temporally precise manner

1. Use an additional computer to control the device, and to communicate with the imaging microscope software to coordinate odor stimulations with image capture during experiments.

2. Initiate a pre-programmed macro package capable of linking the image acquisition software and odor delivery program (e.g., a VBA macro package installed in the microscope control software, see Table of Materials) that is responsive to an external input trigger provided by the initiation of an odor delivery protocol in a separate program).

2. Start the measurement by monitoring "pre-training"/naîve odor-evoked calcium transients at a resolution of $512 \times 512 \mathrm{px}$ and a frame rate of $4 \mathrm{~Hz}$. Deliver a $2.5 \mathrm{~s}$ odor stimulus flanked by additional image acquisition for $6.25 \mathrm{~s}$ preceding odor onset (to establish an $F_{0}$ baseline value) and $12.5 \mathrm{~s}$ after odor offset. Repeat this with a second odorant and then with a third odorant.

3. Continue 3 min after this measurement with classical conditioning ("training") the fly.

1. Select one of the odors presented in the "pre-training" phase to become the $\mathrm{CS}^{+}$odor and another to become the $\mathrm{CS}^{-}$odor. Present the $\mathrm{CS}^{+}$odor using the computer-controlled odor-deliver system for $60 \mathrm{~s}$ alongside twelve $90 \mathrm{~V}$ electric shocks.

2. After a $60 \mathrm{~s}$ break, present the $\mathrm{CS}^{-}$odor alone for $60 \mathrm{~s}$. Use as odorants 4-methylcyclohexanol and 3-octanol. Do not present the third odorant (e.g., 1-octen-3-ol) during this training as it is used as control only before (step 4.2.) and after (step 4.4) the training phase.

4. Measure the "post-training" odor-evoked calcium transients again by repeating the "pre-training" odor stimulation protocol (step 4.2.) 3 min after finishing the training phase (step 4.3)

NOTE: The timing of this step should reflect the time of interest after memory formation (e.g., carry out this step 3-4 min after the conditioning step to investigate short-term memory). Typically, flies can survive for several hours in this preparation.

5. Save imaging files in an appropriate format (e.g., Tiff) for later image analysis.

\section{Image analysis}

1. To analyze images, open Tiff (or similar) files in the image analysis software such as $\mathrm{Fiji}^{27}$

2. Align the stacks using a movement correction algorithm to ensure minimal movement in the $X$ and $Y$ directions. Discard any recordings that show strong movement in the $\mathrm{Z}$ axis.

3. Select the tool of the software used to mark a region of interest (ROI) around the area that is to be examined. This should be as precise as possible to limit the influence of background fluorescence.

4. Use the respective tool of the software to extract temporal fluorescence intensity data as data files from the selected ROI, such that a value is generated for each frame of the recording.

5. Open the data using a data analysis program to calculate the $\Delta F / F_{0}$ values for each odor trace. $F_{0}=$ average fluorescence in the $2 \mathrm{~s}$ before odor onset. $\Delta F=$ the difference between the raw fluorescence in a given frame and $F_{0}$. From these values, calculate a $\Delta F / F_{0} v a l u e$ for each frame which can be plotted against time to reflect relative fluorescence throughout the odor stimulus period.

\section{Representative Results}

An example of images acquired with the above protocol can be seen in Figure 2. dHomer-GCaMP3 is expressed in an MB output neuron whose dendrites innervate the compartment 1 of the MB Y-lobe (the neuron is termed MVP2 ${ }^{28,29}$ ) and is genetically targeted using the split-Gal4 line $\mathrm{MB} 112 \mathrm{C}^{16}$. Also, demonstrated is the difference in the subcellular localization of a cytosolic and the post-synaptically localized calcium indicator. When comparing Figures 2a and Figure 2f, one can clearly observe the specific, compartmentalized expression of the dHomer-GCaMP3 sensor ${ }^{14}$ - with no expression in the axonal compartments of the neuron, and a punctuated signal visible in the dendritic compartment. Clear - though the lower amplitude - odor responses can be seen in flies expressing $d$ Homer-GCaMP3 (Figure 2, lower panels), compared to flies expressing cytosolic GCaMP6f ${ }^{23}$ (Figure 2, upper panels). This demonstrates that the tool is effective for the visualization of olfactory responses at the level of the postsynapse, and, therefore, provides an additional specificity to the examination of the neural circuits underlying, in this case, odor encoding and olfactory learning 
An example of the latter can be seen in Figure 3. In this experiment, $d$ Homer-GCaMP3 is expressed as in Figure 2 - in the mushroom body output neuron MVP2. This is a neuron known to be modulated through olfactory learning such that presynaptic depression as a result of aversive olfactory conditioning is reflected in a deceased postsynaptic response to the trained odor ${ }^{28,29}$ and here a confirmation of this result is shown using this in vivo calcium imaging protocol. The calcium traces shown in Figure 3 represent exemplary data from one individual fly. Note that the noise level and amplitude can vary between individual preparations (e.g., compare Figure $\mathbf{2 e}$ and Figure $3 \mathrm{c}$-e). Therefore, a within-animal comparison of pre- vs. post-training provides a way to account for inter-individual variability. Of course, the protocol is suitable to be transferred to imaging of other neurons of interest.
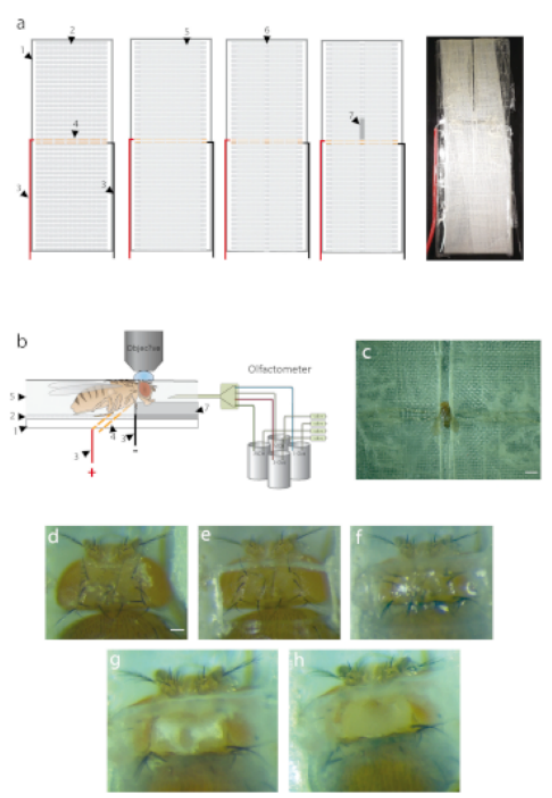

Figure 1: Construction of a mounting chamber and preparation of a fly for imaging. (a) Steps for the construction of the fly mounting chamber. The base is formed of a standard microscope slide (1) covered with a fine plastic mesh (2). Two electrical wires carrying opposing charges (3) are glued to the slide on either side. The wires are stripped and bent to cross the slide (4) running parallel with one another but not in contact. Layers of clear adhesive tape (5) are added until reaching the height of a fly (approximately $1 \mathrm{~mm}$ ). A channel is cut through the tape vertically (6), approximately $1 \mathrm{~mm}$ wide to fit the width of a fly. An elevated platform made of clear adhesive tape (7) is built just above the electrical wires to form a pillow for the fly's head whilst the thorax is on top of the wires. (b) Schematic illustration of the fly positioned under the two-photon microscope. The odor stimulation is achieved through a hypodermic needle positioned in front of the fly's head (inserted through the channel in the tape (6) and on top of the platform (7) to direct the odors to the antennae). (c)-(h) Fixing and opening of the fly head. (c) A fly fixed inside the imaging chamber, inside the channel (Scale bar $=1 \mathrm{~mm}$ ) and held in place by a fresh piece of clear adhesive tape over the whole fly. (d) Magnified view of the fly in position. Scale bar $=0.1 \mathrm{~mm}$. (e) A window is cut into the tape around the head of the fly. (f) The head is further fixed with blue light-curing glue. (g) The head capsule is covered with Ringer's solution and opened. (h) Completed preparation of the brain with excess tissue and trachea (white tissue visible in (g) removed). Please click here to view a larger version of this figure. 


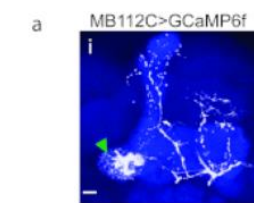

b

$\mathrm{F}_{0}$

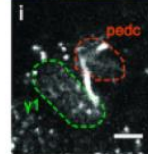

$\mathrm{F}$

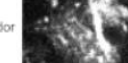

d

$\Delta \mathrm{F}$

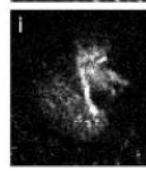

e

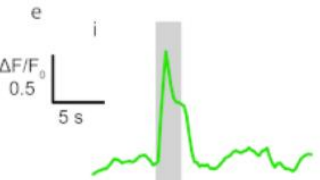

Figure 2: Postsynaptically-localized calcium visualized using $d$ Homer-GCaMP3. (a) Confocal microscopy images showing the expressio of cytosolically localized GCaMP6f (i) and postsynaptically localized dHomer-GCaMP3 (ii) in the MB output neuron MVP2. Green arrow indicates the $\gamma 1$ subregion of the MB $\gamma$-lobe. Scale bars $=15 \mu \mathrm{m}$. (b)-(d) Images captured using two-photon excitation microscopy of the genotypes above, showing the in vivo fluorescence of the respective calcium sensors. (b) $F_{0}$ (baseline fluorescence) images, demonstrated as an average intensity projection of the $2 \mathrm{~s}$ before odor onset. Scale bars $=10 \mu \mathrm{m}$. (c) $\mathrm{F}_{\text {odor }}$ images (fluorescence during odor stimulation), demonstrated as an average intensity projection over the odor response period $(2.5 \mathrm{~s})$. (d) $\Delta \mathrm{F}$ images generated by subtracting $\mathrm{F}_{0}$ image from $\mathrm{F}_{\text {odor }}$ image, to show actual odor response signal. (e) $\Delta F / F_{0}$ traces from the above flies of the respective genotypes through an odor stimulation (grey bar). Please click here to view a larger version of this figure.
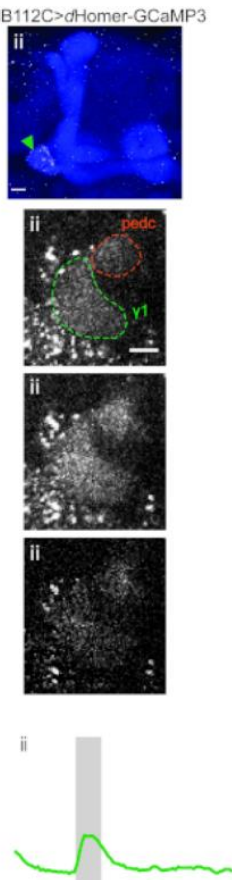


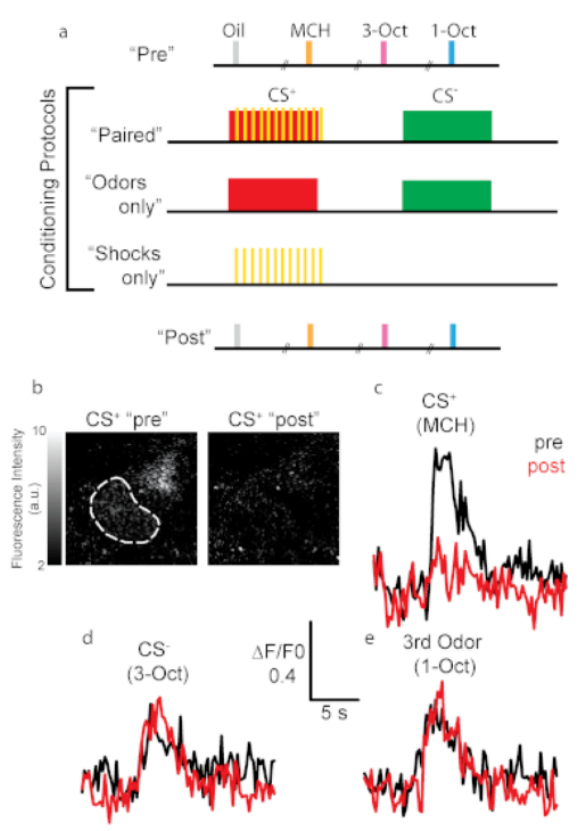

Figure 3: Learning-induced postsynaptic plasticity visualized via $\boldsymbol{d H o m e r - G C a M P 3 . ~ ( a ) ~ S c h e m a t i c ~ o f ~ c o n d i t i o n i n g ~ p r o t o c o l s ~ u s e d ~ i n ~ t h e s e ~}$ experiments. Naïve odor responses, before conditioning ("pre") are measured first. Then each fly experiences one of three possible conditioning protocols: "Paired", where one odor $\left(\mathrm{CS}^{+}\right)$is paired with electric shocks (US) and another (CS") is not reinforced; "Odors only", where only the odors are presented, both without reinforcement, to control for odor exposure effects; and "Shocks only", where only electric shocks are presented without any odor stimuli, to control for shock exposure effects. After this conditioning phase, flies are then exposed to the "Pre" odors again to test for altered odor-evoked activity ("Post"). (b) An example of odor response modulation as a result of the paired odor/shock presentation. Strong suppression of the response to the $\mathrm{CS}^{+}$odor can be seen in the $\mathrm{y}^{1}$ subregion (dotted line). (c)-(e) Odor response traces from the same fly as in (b), showing that this suppression effect is only observed in the case of the trained odor. Please click here to view a larger version of this figure.

\section{Discussion}

The dissection of the neural circuitry underlying learning and memory is a prominent goal in the field of neuroscience. The genetic accessibility of Drosophila and the breadth and ease of behavioral testing makes this an ideal tool to investigate such phenomena. Here, a method is presented with which it is possible to visualize, within individual flies, the modulation that occurs at a subcellular level as a result of olfactory conditioning. By carrying out both pre-training and post-training visualization of odor-evoked calcium dynamics, first established by our group ${ }^{17}$, it is possible to draw a direct, within-animal comparison between naïve and learned odor responses and, therefore, examine the plasticity of neurons of interest. This confers an advantage to this protocol over en masse assessments (used in classical olfactory conditioning) because pre- and post-training states can be quantitatively assessed for individuals, which allows one to determine inter-individual variability. Moreover, the training procedure performed directly under the microscope is largely equivalent to the classical olfactory conditioning paradigm typically used in behavioral experiments and does not involve artificial optogenetic stimulation of neurons. Of course, handling the small flies and carefully opening the head capsule without damaging the brain tissue while keeping the sensory organs intact requires a level of expertise that can be gained by meticulous and repeated training, not unlike the extent seen in physiological assessments such as electrophysiology.

Additionally, the potential to use localized calcium indicators to examine plasticity at the single neuron level is demonstrated - adding greater precision to the neuronal circuit dissection. This is exemplified by showing a learning-induced depression of a postsynaptic response in a wellinvestigated MB output neuron ${ }^{28,29}$. Drosophila strains expressing a variety of synaptically localized fluorescence sensors under UAS control are available, e.g., expressing the presynaptically localized sensor Synaptophysin-GCaMP3 or the red fluorescent sensor of synaptic transmission Synaptophysin-pHTomato ${ }^{20}$. Of course, the variety of fluorescence sensor proteins can be implemented using the protocol described above.

Optical imaging techniques are in general limited by the temporal and spatial resolution of the microscopic image acquisition system (e.g., a twophoton microscope) and the temporal resolution of the sensor itself (e.g., the kinetics of the calcium sensor). Electrophysiological recordings, e.g., using patch clamp electrophysiology from somata of neurons in the Drosophila brain, still offer an unmatched temporal resolution, but without providing any spatial precision. Due to the specificity of the gene expression in neurons of interest combined with the subcellular localization of the sensor, optical imaging techniques can potentially complement electrophysiological techniques to uncover neuronal plasticity underlying learning and memory formation. The continuous progress in the development of fluorescence sensors will perhaps provide the possibility to fuse sensor proteins with faster kinetics or higher signal-to-noise ratios (e.g., GCaMP6 variants ${ }^{23}$ ) with synaptically localized proteins like $d$ Homer. Also, recent advances in establishing microscopic techniques with faster acquisition rates or higher spatial resolution will prove invaluable in further fine-tuning of our approach. 


\section{Disclosures}

The authors have nothing to disclose.

Acknowledgments

This work was supported by the German Research Council through the Collaborative Research Center SFB 889 "Mechanisms of Sensory Processing" and the Research Unit FOR 2705 "Dissection of a Brain Circuit: Structure, Plasticity and Behavioral Function of the Drosophila Mushroom Body".

\section{References}

1. Poo, M.M., et al. What is memory? The present state of the engram. BMC Biology. 14, 40 (2016)

2. Martin, S.J., Grimwood, P.D., Morris, R.G. Synaptic plasticity and memory: an evaluation of the hypothesis. Annual Review of Neuroscience. 23, 649-711 (2000)

3. Takeuchi, T., Duszkiewicz, A.J., Morris, R.G. The synaptic plasticity and memory hypothesis: encoding, storage and persistence Philosophical Transactions of the Royal Society London B: Biological Sciences. 369 (1633), (2013)

4. Josselyn, S.A., Frankland, P.W. Memory Allocation: Mechanisms and Function. Annual Review of Neuroscience. 41, 389-413. (2018).

5. Chiang, A.S., et al. Three-dimensional reconstruction of brain-wide wiring networks in Drosophila at single-cell resolution. Current Biology. 21 (1), 1-11. (2011).

6. Quinn, W.G., Harris, W.A., Benzer, S. Conditioned behavior in Drosophila melanogaster. Proceedings of the National Academy of Sciences of the United States of America. 71 (3), 708-712 (1974).

7. Heisenberg, M., Borst, A., Wagner, S., Byers, D. Drosophila mushroom body mutants are deficient in olfactory learning. Journal of Neurogenetics. 2 (1), 1-30. (1985)

8. de Belle, J.S., Heisenberg, M. Associative odor learning in Drosophila abolished by chemical ablation of mushroom bodies. Science. 263 (5147), 692-695 (1994).

9. Gerber, B., Tanimoto, H., Heisenberg, M. An engram found? Evaluating the evidence from fruit flies. Current Opinion in Neurobiology. 14 (6), 737-744 (2004).

10. Fiala, A., Riemensperger, T. Localization of a memory trace: aversive associative olfactory learning and short-term memory in Drosophila. In: Learning and Memory: A Comprehensive Reference, Vol. 1, 2nd edition., R. Menzel and J.H. Byrne, editors (Oxford: Academic Press.), 475-482 (2017)

11. Cognigni, P., Felsenberg, J., Waddell, S. Do the right thing: neural network mechanisms of memory formation, expression and update in Drosophila. Current Opinion in Neurobiology. 49, 51-58. (2018).

12. Hige, T. What can tiny mushrooms in fruit flies tell us about learning and memory? Neuroscience Research. 129, 8-16. (2018)

13. Aso, Y., Grübel, K., Busch, S., Friedrich, A.B., Siwanowicz, I., Tanimoto, H. The mushroom body of adult Drosophila characterized by GAL4 drivers. Journal of Neurogenetics. 23 (1-2), 156-172. (2009).

14. Pech, U., Pooryasin, A., Birman, S., Fiala, A. Localization of the contacts between Kenyon cells and aminergic neurons in the Drosophila melanogaster brain using SplitGFP reconstitution. Journal of Comparative Neurology. 521 (17), 3992-4026. (2013).

15. Aso, Y., et al. The neuronal architecture of the mushroom body provides a logic for associative learning. Elife. 3, e04577. (2014).

16. Aso, Y., et al. Mushroom body output neurons encode valence and guide memory-based action selection in Drosophila. Elife. 3, e04580. (2014).

17. Riemensperger, T., Völler, T., Stock, P., Buchner, E., Fiala, A. Punishment prediction by dopaminergic neurons in Drosophila. Current Biology. 15 (21), 1953-1960 (2005).

18. Venken, K.J., Simpson, J.H., Bellen, H.J. Genetic manipulation of genes and cells in the nervous system of the fruit fly. Neuron. 72 (2), 202-230. (2011).

19. Riemensperger, T., Pech, U., Dipt, S., Fiala, A. Optical calcium imaging in the nervous system of Drosophila melanogaster. Biochimica et Biophysica Acta. 1820 (8), 1169-1178. (2012).

20. Pech, U., Revelo, N.H., Seitz, K.J., Rizzoli, S.O., Fiala, A. Optical dissection of experience-dependent pre- and postsynaptic plasticity in the Drosophila brain. Cell Reports. 10 (12), 2083-2095. (2015).

21. Tian, L., et al. Imaging neural activity in worms, flies and mice with improved GCaMP calcium indicators. Nature Methods. 6, 875-881. (2009).

22. Nakai, J., Ohkura, M., Imoto, K. A high signal-to-noize $\mathrm{Ca}(2+)$ probe composed of a single green fluorescent protein. Nature Biotechnology. 19 (2), 137-141 (2001).

23. Chen, T.W., et al. Ultrasensitive fluorescent proteins for imaging neuronal activity. Nature. 499 (7458), 295-300. (2013).

24. Estes, P.S., Roos, J., van der Bliek, A., Kelly, R.B., Krishnan, K.S., Ramaswami, M. (1996). Traffic of dynamin within individual Drosophila synaptic boutons relative to compartment-specific markers. The Journal of Neuroscience. 16 (17), 5443-5456. (1996).

25. Brand, A.H., Perrimon, N. Targeted gene expression as a means of altering cell fates and generating dominant phenotypes. Development $118(2), 401-415$ (1993).

26. Dipt, S., Riemensperger, T., Fiala, A. Optical calcium imaging using DNA-encoded fluorescence sensors in transgenic fruit flies, Drosophila melanogaster. Methods in Molecular Biology. 1071, 195-206. (2014).

27. Schindelin, J., et al. Fiji: an open-source platform for biological-image analysis. Nature Methods. 9 (7), 676-682. (2012)

28. Hige, T., Aso, Y., Modi, M.N., Rubin, G.M., Turner, G.C. Heterosynaptic Plasticity Underlies Aversive Olfactory Learning in Drosophila. Neuron. 88 (5), 985-998. (2015)

29. Owald, D., et al. Activity of defined mushroom body output neurons underlies learned olfactory behavior in Drosophila. Neuron. 86 (2), 417-427. (2015). 


\subsection{Discussion}

With this work we have demonstrated a technique whereby learning-induced changes in subcellular calcium dynamics can be visualised in real time. Pavlovian-style classical conditioning in Drosophila is a long-established phenomenon (Quinn et al., 1974; Tully \& Quinn, 1985), and the neural substrates of the learned behavioural adaption observed as a result of that conditioning have been the subject of extensive experimental scrutiny.

In this protocol, we used an adapted version of the classic aversive olfactory associative conditioning whereby a single fly is placed under the microscope with the head capsule open. The fly is then presented with a panel of odour stimuli, and the odour-evoked calcium changes in neurons of interest is monitored and quantified. By then training the fly to associate one of those odours with a punishing electric shock and, after 3-4 minutes, again quantifying odour-evoked calcium dynamics, it is possible to directly observe how neural activity patterns are modulated in response to an odour that is now predictive of a painful punishment. This facilitates the direct visualisation of how neural representations of an odour are modified by the process of learning.

By utilising the further spatial restriction enabled by the use of a postsynaptically localised calcium indicator - homer-GCaMP - the technique presented here provides a way in which learning-induced changes in calcium signalling can be restricted not only to single neurons, but to the primary input sites to those neurons. This provides an advantage over typical genetically encoded indicators that are expressed throughout the cytosol for the precise localisation of where plastic changes are occurring. 


\section{Learning-induced Modulation of Mushroom Body Output Neuron Postsynapses}

\subsection{Introduction and aims}

In this section, we used the methodology described in section 3 to investigate the modulation of a specific population of mushroom body innervating neurons called mushroom body output neurons (MBONs). As their name implies, these neurons lie directly downstream of the mushroom body intrinsic neurons (Kenyon cells, KCs). When an odour is detected by olfactory sensory neurons and that information is relayed to the mushroom body by olfactory projection neurons, a small percentage of the KCs become active. In the case of classical olfactory associative learning, coincidence between this olfactory input and input from dopaminergic neurons conveying rewarding or punishing stimuli leads to a modification of the behavioural response to the associated odour, reflective of the learned positive or negative valence. This change in behavioural response is hypothesised to be mediated by plasticity at the KC-to-MBON synapses, such that odour-evoked input to the MBONs is either depressed or facilitated.

Indeed, evidence has mounted in recent years that corroborates this theory. Electrophysiological (Hige, Aso, Modi, et al., 2015) and optical imaging (Berry et al., 2018; Owald et al., 2015; Perisse et al., 2016) studies have shown that many MBONs show learning-mediated modulation of their activity in response to presentation of odours learned to be predictive of either rewarding or punishing stimuli (e.g. sugar or electric shock, respectively). This is posited to be due to a role for MBONs in encoding stimulus valence such that, dependent on the region of the mushroom body they innervate, some MBONs are linked to stimulus approach while others are linked to stimulus avoidance (Aso et al., 2014b).

This study took a novel approach to investigating the role of MBONs in encoding learned odours. The role of the KCs that form the mushroom body $\mathrm{y}$-lobe in olfactory learning and memory is well documented (Blum et al., 2009; Zars et al., 2000a). Specifically, the synaptic output from these neurons is particularly important at the point of short-term memory recall (Dubnau et al., 2001). It therefore seems intuitive that the receipt of $\gamma \mathrm{KC}$ input (i.e., at the MBON postsynapse) represents an important gauge in deciphering the valence of a learned odour.

Thus, in the following section we used the techniques and postsynaptically-localised calcium indicator described in section 4 to probe the effects of aversive olfactory conditioning on the odour-evoked activity of the five MBONs that receive input from the mushroom body $\mathrm{YKCs}$. With this approach, we 
aimed to achieve a more comprehensive analysis of the role of the $\mathrm{\gamma}$-lobe MBONs in reading out a memory trace from the mushroom body.

\subsection{Results}

\subsubsection{Localisation of homer-GCaMP to the mushroom body output neuron post-synapse}

First, we confirmed the localisation of the homer-fused GCaMP to the post-synaptic compartment of each of the $\psi$-lobe MBONs. Brains of flies expressing either cytosolic GCaMP6f or homer-GCaMP-fused GCaMP3 in single MBONs were explanted and subjected to immunohistochemical staining. To target each of the MBONs individually, specific split Gal4 driver lines were used. Less fluorescence overall was visible from MBONs expressing homer-GCaMP as compared to cytosolic GCaMP6f (Figure 4.1., A), with little or no fluorescence signal in the axonal compartments of the neurons. Indeed, as previously reported (Pech et al., 2015), the homer-fused GCaMP was primarily localised in the dendritic compartments of the MBONs. Furthermore, this sensor also displayed a comparatively punctuated signal in these regions, with minimal signal emanating from the larger neurites within the dendritic compartments (visible in GCaMP6f-expressing neurons). This is conducive with the putative localisation of the GCaMP principally at the postsynaptic sites to which Homer is trafficked (Pech et al., 2015).

This localisation was also notable when visualised in vivo using two-photon microscopy (Figure 4.1., B). Again, the baseline fluorescence of the cytosolic GCaMP6f was more intense and predominated by the presence of larger neurites innervating the MB compartment (in the case of Figure 4.1., B, the $\gamma 1$ compartment). By comparison, homer-GCaMP again exhibited a more punctuated and precisely localised signal.

These differences were also evident when the flies were presented with odour stimuli (Figure 4.1., C). Although, it should be noted that due to the different sensors used to detect calcium in these cases GCaMP3 vs. GCaMP6f - and the observed fluorescence signal is variable between these sensors. Of importance for this study, above this, is that the odour-evoked changes in fluorescence observed using homer-GCaMP are more than robust enough to be easily visualised and quantified in further experiments. Moreover, the odour identity-dependent variability in response amplitude is consistent between the two sensors - i.e., in both cases one can observe a more robust odour-evoked calcium influx in response to $\mathrm{MCH}$ than 3-Octanol, for example (Figure 4.1., C). 
A) $\begin{aligned} & \text { Cytosolic } \\ & \text { (GCaMP6f) }\end{aligned}$
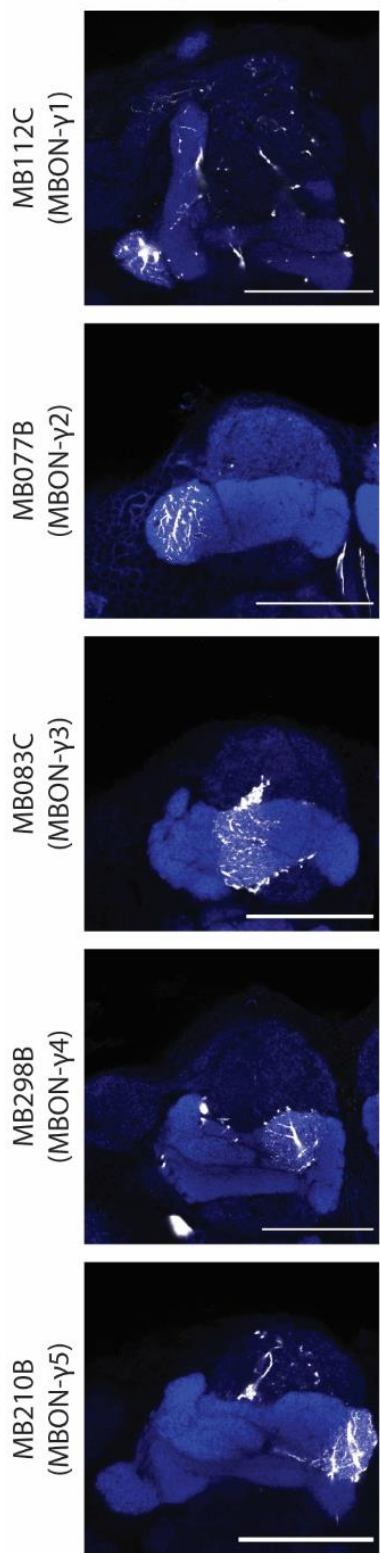

Postsynaptic (dhomer-GCaMP3)
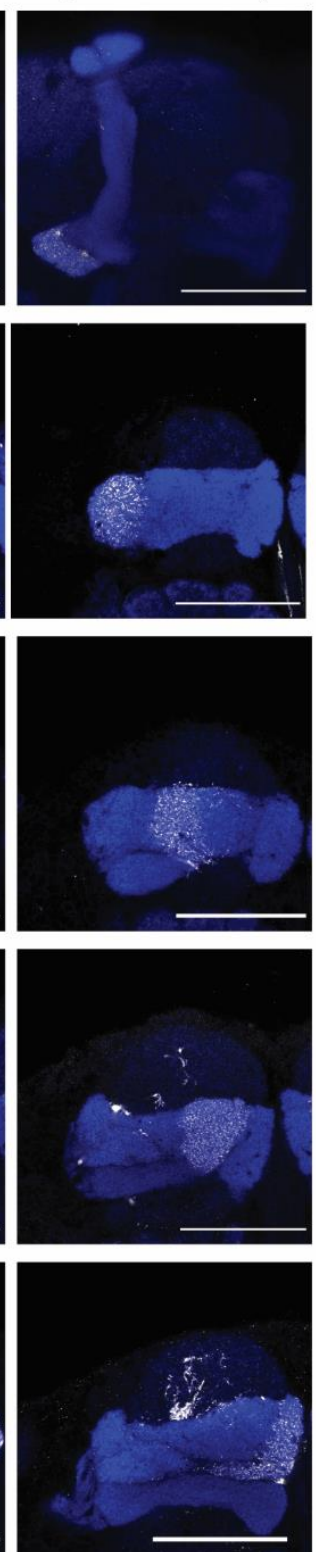

B)

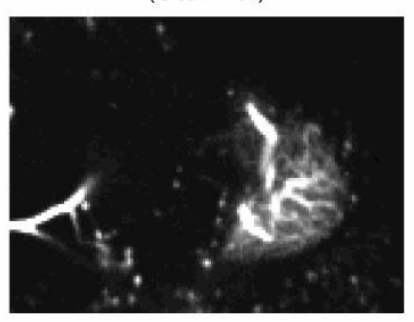

C)
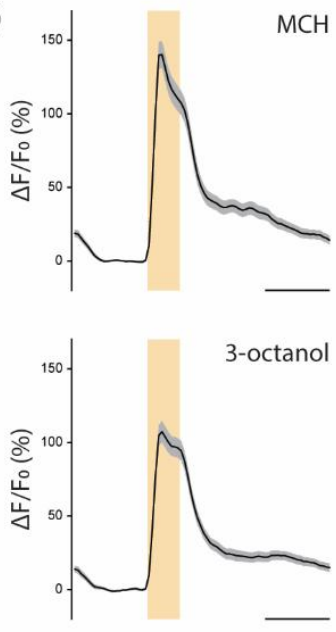

Postsynaptic (dhomer-GCaMP3)

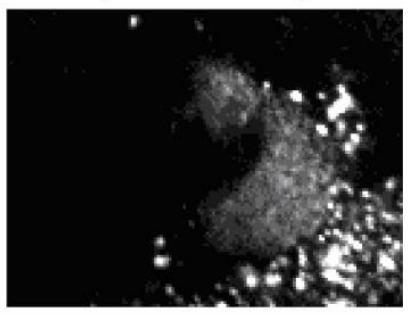

$\mathrm{MCH}$
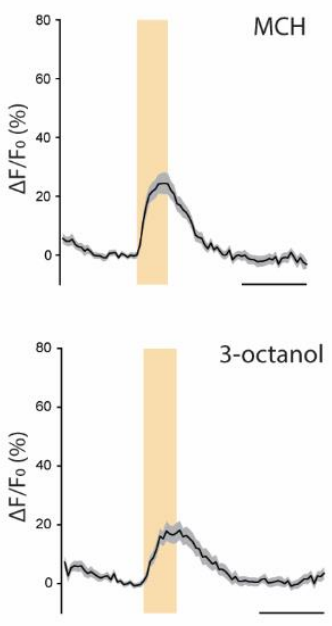

Figure 4.1.: Cytosolic vs. postsynaptically-localised GCaMP. (A) Confocal anatomical images showing the expression of genetically encoded calcium indicators GCaMP6f (cytosolic, left column) and homer-GCaMP (postsynaptic, right column), under the control of specific split-Gal4 drivers for each of the MBONs. Scale bars $=50 \mu \mathrm{m}$. (B) The dendritic arbours of MBON- $\gamma 1$ in the $\gamma 1$ compartment, visualised in vivo using two-photon microscopy, using either GCaMP6f or homer-GCaMP. (C) Average odour-evoked response measured using either GCaMP6f (left) or homer-GCaMP (right) for two different odours ( $\mathrm{MCH}$ and 3-Octanol) used throughout this study. Lines indicate means, shaded areas indicate SEM. For GCaMP6f, $n=119$ flies, for homer-GCaMP $n=30$ flies. Scale bars $=5$ sec.

\subsection{2. $\mathrm{MBONs}$ of the $\mathrm{MB} \mathrm{Y}$-lobe receive heterogenous odour drives}

In order to address the question of how the $\mathrm{\gamma}$-lobe MBONs encode odours, we next exposed flies to a simple odour delivery protocol while monitoring homer-GCaMP fluorescence from single MBONs. Therein, the heads of living flies were opened to expose the brain and visualise MBON dendritic compartments using two-photon microscopy. Flies were then presented with three different odorants - MCH, 3-Octanol, and 1-Octen-3-ol - whilst emitted GFP signal was visualised and recorded. The 
MBONs innervating compartments 1-4 all showed robust odour-evoked responses, although of variable amplitude and differing degrees of inter-individual variability (Figure 5.2.). Responses, for example, measured from the $\gamma 3$-innervating MBON- $\gamma 3 \beta^{\prime} 1$ showed, on average, much higher amplitude responses than the other MBONs. In contrast, $\gamma 1,2$, and 4 MBONs showed quite similar response dynamics with $\gamma 2$ and $\gamma 4$ being very similar in both amplitude and temporal qualities and $\gamma 1$ in general responding with a lower amplitude.
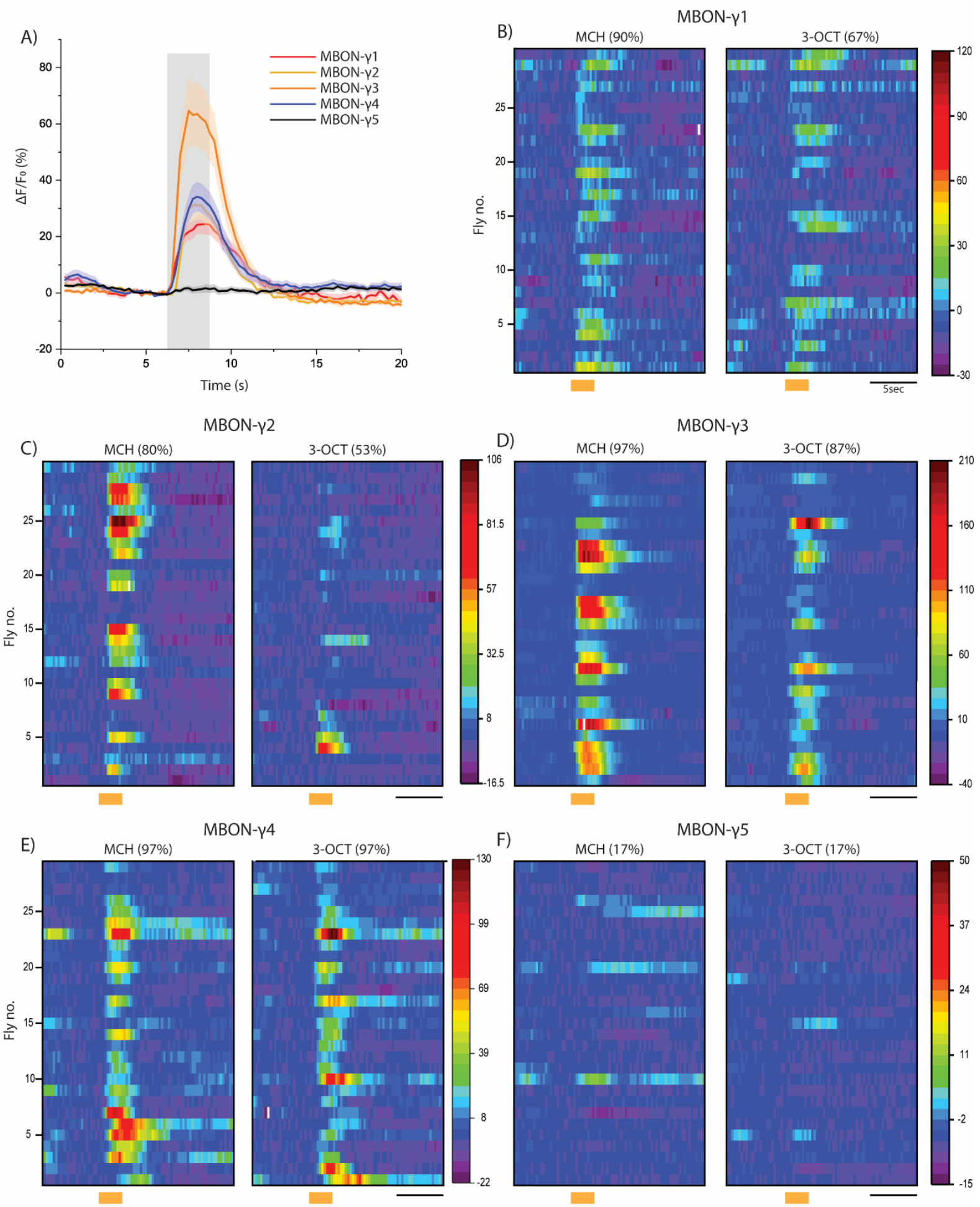
Figure 4.2.: Naïve odour responses across $\gamma$-MBONs. (A) Mean response traces for each of the MBONs investigated, following presentation of $\mathrm{MCH}$. Lines indicate mean $\triangle \mathrm{F} / \mathrm{FO}$ values over time, and shaded areas indicate SEM. For each MBON, n=29-30 flies. Grey bar indicates odour delivery period. (B)-(F) Heatmaps showing naïve responses to $\mathrm{MCH}$ and 3-Octanol, across all flies (rows) measured for each MBON. Percentage of measured flies that showed an odour response is stated above each heatmap. Orange box indicates odour delivery timing, bottom right scale bar $=5 \mathrm{sec}$.

These four MBONs also showed similar response probabilities to the two test odours described - $\mathrm{MCH}$ and 3-Octanol (Figure 4.2., B-E). A response here was defined as a $\Delta F / F_{0}$ value during or immediately following odour presentation that exceeded three times the standard deviation of the baseline. In each case, between $80-97 \%$ of cells imaged across flies showed a significant response to $\mathrm{MCH}$. Conversely, 3-Octanol, in general, evoked a response in fewer observed MBONs in each group, except the $\gamma 4$-innervating $\mathrm{MBON}-\mathrm{\gamma} 4$ in which almost all cells measured responded to both odours (Figure 4.2., E). Notably, only $57 \%$ of $\gamma 2$ MBONs showed a significant response to 3-Octanol. This demonstrates that, although each of the MBONs of the $\gamma$-lobe has, in theory, the potential to receive input from all $\mathrm{KCs}$ of the $\mathrm{y}$-lobe and are therefore exposed to very broad olfactory inputs, their response profiles are variable and dependent on the odour identity.

A prominent exception to the above trends was the near-absolute lack of odour-evoked activity seen in the $\gamma 5$-innervating MBON (Figure 4.2., $\mathrm{A}$ and $\mathrm{F}$ ). This phenomenon has been previously reported in similar experimental set-ups (Hige, Aso, Rubin, et al., 2015; Perisse et al., 2016). Indeed, only approximately $17 \%$ of cells measured -5 cells out of 30 - showed significant calcium increase in response to $\mathrm{MCH}$ or 3-Octanol (Figure 4.2., F). The MBON from which these measurements were recorded innervates multiple compartments of the $M B$, including multiple subcompartments of the $\beta^{\prime}$ lobe as well as the $\mathrm{\gamma 5}$ region. Owald et al. (2015), upon finding no odour-evoked activity in the latter, instead conducted all measurements from the $\beta^{\prime} 2$ compartment of the $\mathrm{MBON}(\mathrm{s})$, where responses are robust. Due to our explicit interest in the activity of the $\gamma$-lobe, the recordings that we acquired in this study were measured only from the portion of the $M B O N(s)$ that occupies the $\gamma 5$ region.

\subsubsection{Aversive associative conditioning leads to suppression of $\mathrm{MBON}-\gamma 1$ at the postsynapse}

We next wanted to investigate how the odour-evoked calcium dynamics that we observed are influenced by the process of associative olfactory conditioning. To test this, we subjected flies to a protocol in which one of our test odours was presented together with pulsing electric shocks to the body of the fly ( $1290 \mathrm{~V}$ shocks over 60 seconds). The associated odour is then termed the "conditioned stimulus +", or CS+, odour. After a break of 60 seconds, the flies were then presented with a second 
of our test odours for 60 seconds with no other stimulus. This odour is then the "conditioned stimulus -", or CS-, odour (see Figure 4.1.). Such a protocol has been demonstrated to lead to robust avoidance of the CS+ odour in behavioural assays, as the animal learns that that specific odour is predictive of a punishment and should therefore be avoided in favour of the CS- odour (Tempel et al., 1983; Tully \& Quinn, 1985). In these experiments, we sought to investigate how the odour drive to the different MBONs along the MB $\gamma$-lobe is modulated through the process of learning. Therefore, we visualised the odour-evoked activity of each MBON before and after exposing the flies to the aversive associative conditioning protocol described above.

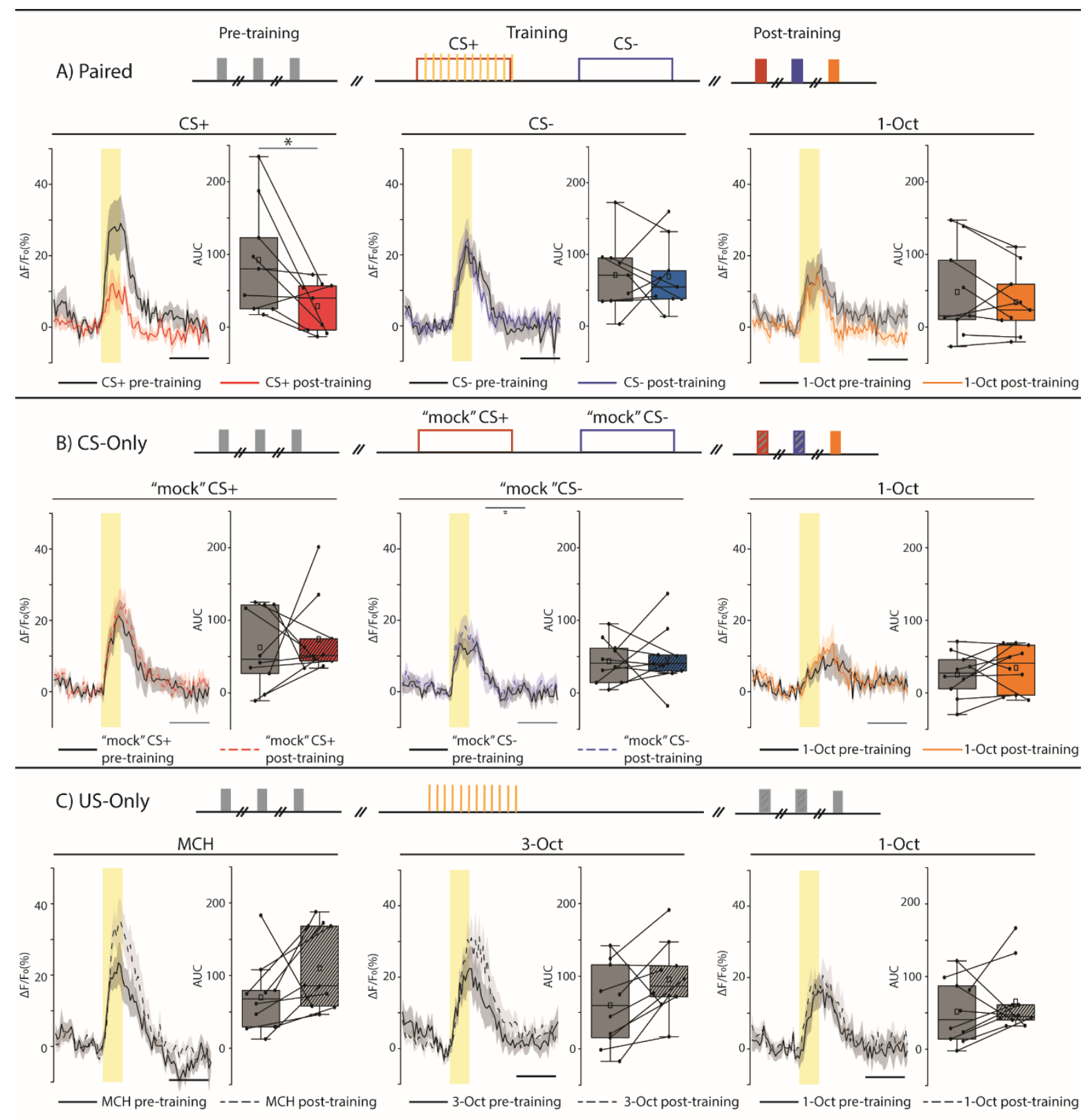

Figure 4.3.: Learning-induced suppression of postsynaptic odour-evoked calcium transients in MBON- $\gamma 1$. Odour-evoked activity before and after the three different training protocols used: Paired 
(A), in which one odour (CS+) is paired with electric shock, and one odour is presented alone (CS-); CSOnly (B) in which two odours are presented, neither paired with electric shock, to control for prolonged odour presentations; and US-Only (C) in which no odours are presented during the training phase, but the standard electric shock procedure is delivered to control for effects of electric shock. In the paired and CS-Only groups, $\mathrm{MCH}$ and 3-Octanol were used reciprocally in the training stage. 1Oct was always presented in the pre-training and post-training measurements, but never during training. For each odour condition, traces represent mean $\Delta F / F_{0}$ values over time and shaded areas show SEM. Box plots show the area under curve (AUC) values for all flies under each condition, depicting upper and lower quartiles and median. Whiskers represent $95 \%$ confidence interval. For paired group, $n=9$ flies, for CS-only group $n=10$ flies, for US-Only groups $n=10$ flies. Pre-to-post changes were statistically tested using the paired Wilcoxon signed ranks test $\left({ }^{*} \mathrm{p}<0.05\right)$.

The effects of aversive olfactory conditioning on the odour responses of MBON- $\psi 1$ have been demonstrated in previous publications: pairing an odour with an aversive US - either in the form of electric shock or by optogenetic activation of the neurons that represent the presence of electric shock

- leads to a relative reduction in the response of this MBON to the paired odour (CS+), compared to an unpaired odour (CS-) (Hige, Aso, Modi, et al., 2015; Perisse et al., 2016). Indeed, we also observed a depression of MBON- $\gamma 1$ in response to the CS+ odour after the conditioning protocol (Figure 4.3., A). This depression occurred only in the case of the CS+ odour and was not the case for the CS- odour or in either of the control groups (Figure 4.3., B and C), or for the control odour 1-Oct that was presented only in the pre- and post-training recording periods. In fact, we additionally observed an increase in $\mathrm{MBON}-\gamma 1$ response to two of our experimental odours ( $\mathrm{MCH}$ and 3-Octanol) following a protocol in which flies received only the 60 second electric shock protocol (Figure 4.3., C).

Given the putative positive valence coding of MBON- $\psi 1$ (Aso et al. 2014b), these results appear cohesive with the valence-shifting hypothesis whereby assigning a positive or negative value to a previously neutral odour leads to a shift in the activity of the MBON network to favour an appropriate behavioural output. In this case, a reduction in the activity of MBON- $\gamma 1$ in response to an aversively conditioned odour would, in theory, lead to reduced approach behaviour when that odour is detected in the future.

\subsubsection{MBONs- $\gamma 2-5$ show no detectable postsynaptic memory trace}

This specific, learning-induced modulation of odour responses was not observed in any of the further $M B$ Y-lobe MBONs. Instead, we found that, in most cases, odour responses in the MBONs innervating ү2- y4 compartments all show a reduction in odour-evoked activation in the post-training measurement, independent of the type of protocol they were exposed to (Figure 4.4.). This appears 
to predominantly be a form of neural adaptation, whereby the extended exposure to the odours during the training step leads to a reduced responsiveness in the post-training phase. This is supported by the appearance of much weaker suppression of responses in the case of the shock-only control groups - in which there is no prolonged odour exposure (Figure 4.4., rightmost columns).

It is also notable that the degree of adaptation is variable between the different neuron types. For example, the depression seen in MBON- $\gamma 4$ is not as robust as in the $\gamma 2$ and $\gamma 3$ MBONs (Figure 4.4., C). Indeed, in the latter two cases there appears to be an almost complete suppression of odour responses in the odour-only control groups. This perhaps suggests that the different MBONs of the $\mathrm{MB} Y$-lobe possess differing adaptive characteristics, such that the level of neural adaptation that we observe here as a result of prolonged odour exposure is variable between neuron types.

Nonetheless, from these data it appears that, beyond the $\gamma 1$ compartment of the $M B$, there is no specific, learning-induced modulation of the odour-evoked input to the MBONs of the $\mathrm{Y}$-lobe. This would appear to be in contradiction to the valence-shifting model popularised in recent years whereby all $5 \mathrm{MBONs}$ would undergo a rebalancing in their overall activation such that the "avoidance coding" MBONs of the $\gamma 4$ and $\gamma 5$ region would show an increase in their response to an aversively paired odour and the "approach coding" MBONs of the $\psi 1,2$, and 3 regions would, in parallel, show a reduction in activity. Instead, we demonstrate that, at the level of the MBON post-synapse - that is, the putative point of direct input from the odour-coding Kenyon cells - there is limited plasticity to be found along most of the MB $\mathrm{Y}$-lobe. It is important to note that previous studies that find learninginduced changes in other MBONs - such as in MBON- $\nu 2$ and MBON- -5 - used different methodology, either in the use of a cytosolically expressed calcium indicator or in the measurement of spatially distinct dendritic arbours, respectively (Berry et al. 2018; Owald et al. 2015). 


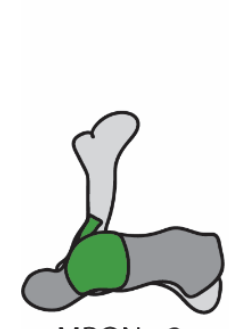

MBON- $\gamma 2$
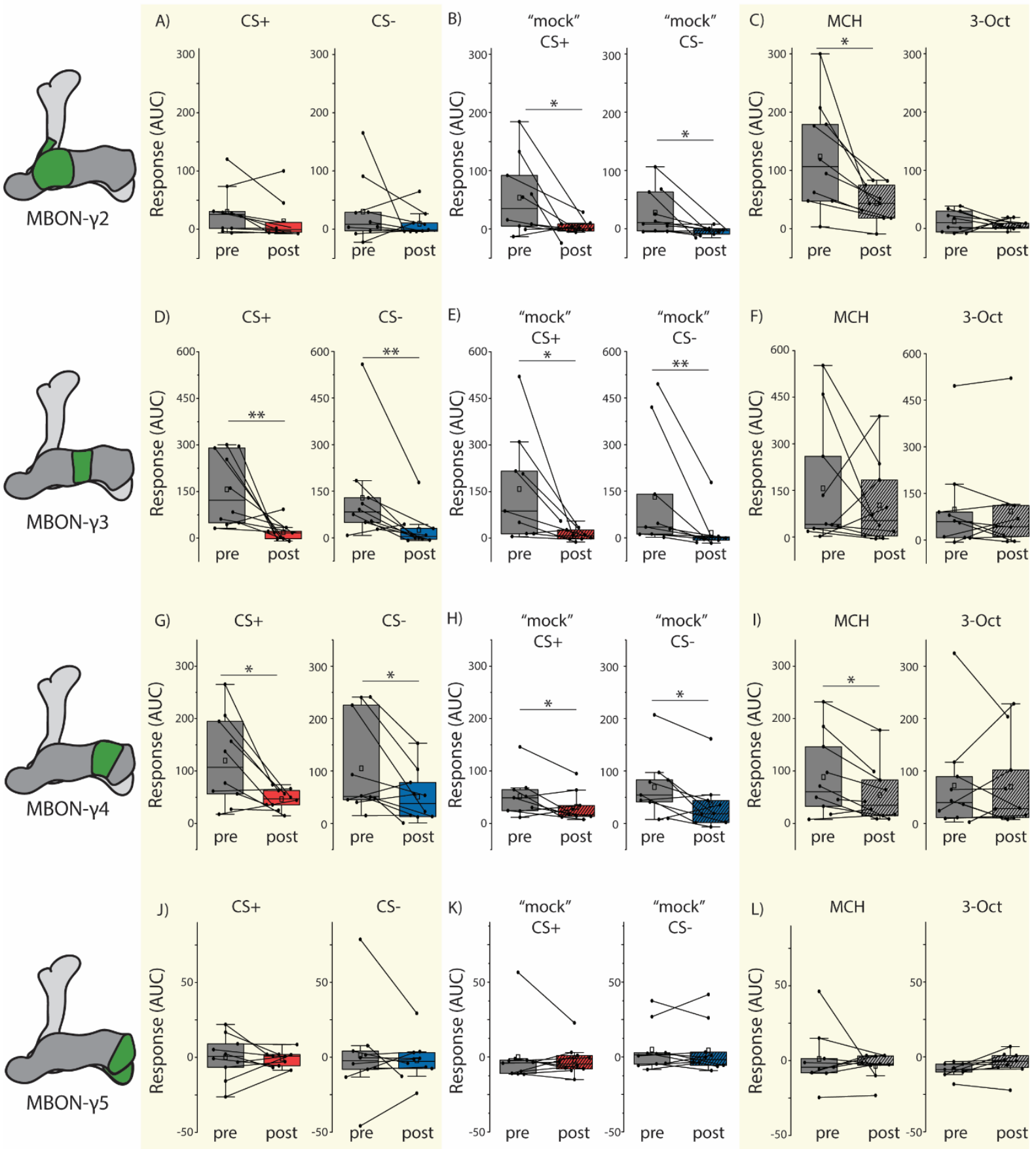

Figure 4.4.: Non-associative effects of different training procedures in MBONs innervating compartments $\gamma 2-\gamma 5$. Odour-evoked responses measured in each of the MBONs innervating the $\gamma 2$ (A-C), y3 (D-F), y4 (G-I), and y5 (J-L) compartments. Box plots show quantifications of responses as area under curve values calculated for each response trace (mean traces can be found in the appendix (12.1.)). Schematics of the anatomical position of dendritic arbours for each neuron can be seen in the left-most column. Boxes show median and upper and lower quartiles. Data points represent responses before training ("pre") and after training ("post"), with responses from the same fly connected by lines. Significant changes from pre-to-post were tested using the paired Wilcoxon signed rank test $\left({ }^{*} p<0.05,{ }^{* *} p<0.01\right)$. 


\subsection{Discussion}

Previous studies into the neural circuits underlying associative learning in Drosophila have identified many of the key players involved. Indeed, the primary brain region linked to this process - the mushroom body $(\mathrm{MB})$ - has been under intensive experimental observation for decades and is believed to be the structure in which learning-induced synaptic plasticity occurs (e.g., reviewed by (Cognigni et al., 2018; Heisenberg, 2003). What has not been clear is how this plasticity is translated readout and conferred downstream. After all, the generation of a signal is only of use if it can be aptly received and processed.

The work presented here aimed to address this query by examining the primary downstream targets of the $\mathrm{MB}$ - the mushroom body output neurons (MBONs). Previous studies have linked these neurons to the behavioural expression of preference, whereby artificial activation of some MBONs leads to an approach behaviour while others lead to avoidance (Aso et al., 2014b). This connection between activity of specific MBONs and valence has been further exemplified by functional imaging experiments that showed that the learning of an odour as predictive of punishment (electric shock) caused suppression of "approach" MBONs and the potentiation of "avoidance" MBONs (Berry et al., 2018; Hige, Aso, Modi, et al., 2015; Owald et al., 2015).

By taking a novel approach to further examining the nature of these learning-induced changes in activity, we were able to look more closely at the postsynaptic input sites of the MBONs where we theorised the majority of this plasticity was taking place. This was possible by expressing a genetically encoded, Homer-fused calcium indicator - homer-GCaMP - in each of the $\gamma$-lobe MBONs and monitoring odour-evoked activity before and after odour-electric shock conditioning. In doing so, we found that only one of the MBONs -MBON- 1 - showed training-induced modulation, with responses to the trained odour ( $\mathrm{CS}+$ ) being diminished after pairing. This was not observed under either of the control conditions that do not induce associative learning. The remaining $\mathrm{Y}-\mathrm{MBONs}$, however, displayed only non-associative effects. In fact, our data show that MBONs innervating compartments Y2-4 strongly adapt to the prolonged odour stimuli used in these experiments. As previously reported, MBON- $\gamma 5$ showed extremely scarce odour responses even before conditioning. These findings lead us to conclude that, at the postsynapse level, only the MBON receiving input within the $\gamma 1$ compartment of the MB is subject to learning induced modification.

This finding is interesting in the context of other studies that identify, using cytosolically expressed GCaMP, these types of effects across the $\mathrm{Y}$-MBON population. Indeed, this demonstrates the value of using subcellularly localised sensors in deciphering, with more precision, the neuronal substrates of learning. For example, recent connectomics studies in which their complete three-dimensional 
structure and putative synaptic connections have been identified have shown that a complex multilevel feedback network exists within and around the MB such that many of the MBONs provide feedforward and feedback input to one another (Li et al., 2020b; Takemura et al., 2017). The presence of such networks implies that the plasticity observed in the MBONs at the axonal level or the somata are likely due to other inputs than those from the MB KCs directly. Conversely, the functional plasticity we observe in the $\gamma 1$ compartment may be reflective of a more specialised role for this MBON in learning and memory. It is notable, for example, that the dopaminergic neuron that projects into the $\gamma 1$ compartment - where it provides input to both the KCs and the $\gamma 1 \mathrm{MBON}$ - is part of the PPL1 cluster of dopaminergic neurons that show strong responses to electric shock stimuli like those used here. In fact, omission of the electric shock in favour of optogenetic activation of the $\gamma 1$ dopaminergic neuron is sufficient to induce strong aversive associative learning (Aso \& Rubin, 2016; Hige, Aso, Modi, et al., 2015). Combined with our findings here, this implies a specialised function of the $\gamma 1$ compartment of the MB in aversive short-term memory formation. 


\section{Visualisation of Learning-Induced Modulation of Odour-Evoked Activity at Kenyon Cell Presynaptic Sites}

\subsection{Introduction and aims}

In this section, one can find the publication "Visualization of naive and learned odor representations using in vivo calcium imaging and immunohistochemical bouton mapping of single Drosophila mushroom body neurons". This paper documents, in detail, the methodology used in section 6 . As in the previous sections, this study centres on the use of in vivo calcium imaging in deciphering the processes underlying odour coding and how this is modulated by olfactory learning and memory formation.

The Drosophila mushroom body represents a key brain region in the processing of odour stimuli. Olfactory input from the antennal lobe, via olfactory projection neurons, occurs in the calyx region of the mushroom body, where the dendrites of the mushroom body intrinsic Kenyon cells (KCs) reside. The KCs then extend through the pedunculus and to the lobes of the mushroom body, formed by the axons of the KCs. As in section 4, we focus here specifically on the $\gamma$-lobe due to its connection to short-term memory.

Although all $\gamma \mathrm{KCS}$ project axons through the length of the $\mathrm{\gamma}$-lobe, the tiling innervation pattern of mushroom body extrinsic neurons (discussed in section 4) means that different parts of the same KCs are subjected to differential inputs and give outputs to different cell types. The technique described herein was thus developed with the aim of analysing how odour information is processed in the mushroom body $\mathrm{y}$-lobe in relation to the anatomical position along the $\mathrm{KC}$ axon. To do so, we visualised in vivo odour-evoked calcium dynamics at sites of densely localised presynaptic proteins structures we refer to here as synaptic boutons - and then, using the subsequently explanted and immunostained brain, mapped the anatomical location of each bouton.

Using both the functional and anatomical characteristics of each synaptic bouton, this technique facilitates a more nuanced dissection of how odours are encoded by the mushroom body. In particular, the computational analysis described here - designed and performed by Dr. Bart Geurten - introduces the use of the so-called amplitude-corrected correlation as a measure of the level of congruence between synaptic boutons across the $\gamma$-lobe. With this, one can characterise the neuronal representation of an odour in terms of a dispersed array of active synapses that act more or less cohesively dependent on learned odour valence. 
5.2. Manuscript - Visualization of Naïve and Learned Odor Representations Using in vivo Calcium Imaging and Immunohistochemical Bouton Mapping of Single Drosophila Mushroom Body Neurons

STAR Protocols, December 2020

Authors: Clare E. Hancock*, Bart R.H. Geurten*, André Fiala

*Equally contributing first author

Contribution:

Wrote the manuscript in collaboration with Dr. Bart Geurten, performed experiments and analyses (the results of which can be found in section 7). 


\section{STAR Protocols}

\section{Protocol}

\section{Visualization of naive and learned odor}

representations using in vivo calcium imaging and immunohistochemical bouton mapping of single Drosophila mushroom body neurons

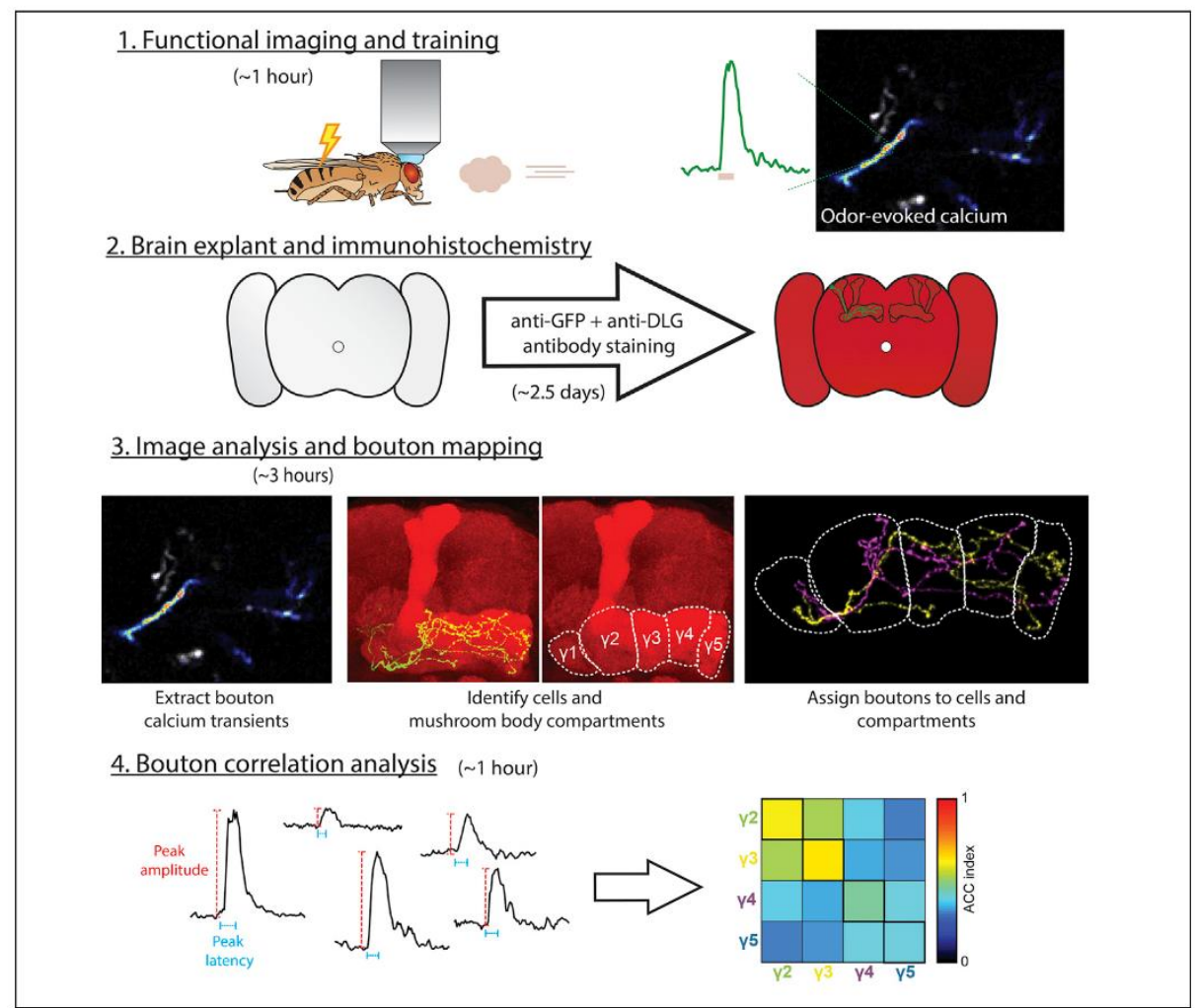

Clare E. Hancock, Bart R.H. Geurten, André Fiala

clare.hancock@ uni-goettingen.de (C.E.H.) bgeurte@gwdg.de (B.R.H.G.) afiala@gwdg.de (A.F.)

\section{HIGHLIGHTS}

Neuronal activity is monitored in vivo at single synaptic bouton level in Drosophila

Learning-induced modulation of calcium dynamics is visualized

Congruence of odor responses is quantified across distributed synapses

This protocol enables the quantification of odor-evoked calcium activity in mushroom body Kenyon cells of the Drosophila melanogaster brain at the single bouton level. We also present subsequent characterization of naive and learned odor representations in the context of olfactory coding. This approach to analyzing the neuronal basis of associative learning provides a substrate for similar studies, perhaps in other animals, to probe the attributes of a neuronal memory trace at the level of synapses distributed across neurons. 
Visualization of naive and learned odor representations using in vivo calcium imaging and immunohistochemical bouton mapping of single Drosophila mushroom body neurons

\author{
Clare E. Hancock, ${ }^{1,3,4, *}$ Bart R.H. Geurten, $2,3,4, *$ and André Fiala $1,5, *$ \\ 1Department of Molecular Neurobiology of Behaviour, Johann-Friedrich-Blumenbach-Institute for Zoology and \\ Anthropology, University of Goettingen, Julia-Lermontowa-Weg 3, 37077 Goettingen, Germany \\ 2Department of Cellular Neurobiology, Johann-Friedrich-Blumenbach-Institute for Zoology and Anthropology, University of \\ Goettingen, Julia-Lermontowa-Weg 3, 37077 Goettingen, Germany \\ ${ }^{3}$ These authors contributed equally \\ ${ }^{4}$ Technical Contact \\ ${ }^{5}$ Lead Contact \\ *Correspondence: clare.hancock@uni-goettingen.de (C.E.H.), bgeurte@gwdg.de (B.R.H.G.), afiala@gwdg.de (A.F.) \\ https://doi.org/10.1016/j.xpro.2020.100210
}

\title{
SUMMARY
}

This protocol enables the quantification of odor-evoked calcium activity in mushroom body Kenyon cells of the Drosophila melanogaster brain at the single bouton level. We also present subsequent characterization of naive and learned odor representations in the context of olfactory coding. This approach to analyzing the neuronal basis of associative learning provides a substrate for similar studies, perhaps in other animals, to probe the attributes of a neuronal memory trace at the level of synapses distributed across neurons.

For complete details on the use and execution of this protocol, please refer to Bilz et al. (2020).

\section{BEFORE YOU BEGIN}

To visualize calcium transients in single Kenyon cells, flies must first be generated that express the required transgenes in a limited number of cells. In this study (Bilz et al., 2020), cells were monitored both functionally (in vivo) and anatomically (ex vivo). The former requires the expression of an activity-dependent, genetically encoded calcium indicator (in this case GCaMP3), whilst the latter was aided by the additional inclusion of a strongly expressed fluorescent cell marker (mCherry). Details of the specific fly strains used in this study can be found in the Key resources table. With the exception of the heat-shock protocol required for the induction of gene expression using the MARCM gene expression system, flies were raised under standard laboratory conditions. For a schematic depiction of the preparatory steps described in this section, see Figures $1 \mathrm{~A}-1 \mathrm{D}$.

Fly strains and husbandry

(c) Timing: $1 \mathrm{~h}$

1. Raise fruit flies in vials containing standard Drosophila food medium in an incubator at $25^{\circ} \mathrm{C}$, relative humidity of $60 \%$, and a controlled $12 \mathrm{~h} / 12 \mathrm{~h}$ light/dark cycle.

2. Collect virgin female flies carrying the following transgenes: neoFRT19A and 5HT1B-Gal4. In parallel, also collect male flies carrying the following transgenes: neoFRT19A, hsFLP, tubP-Gal80, 
A

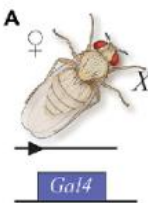

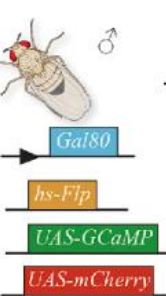
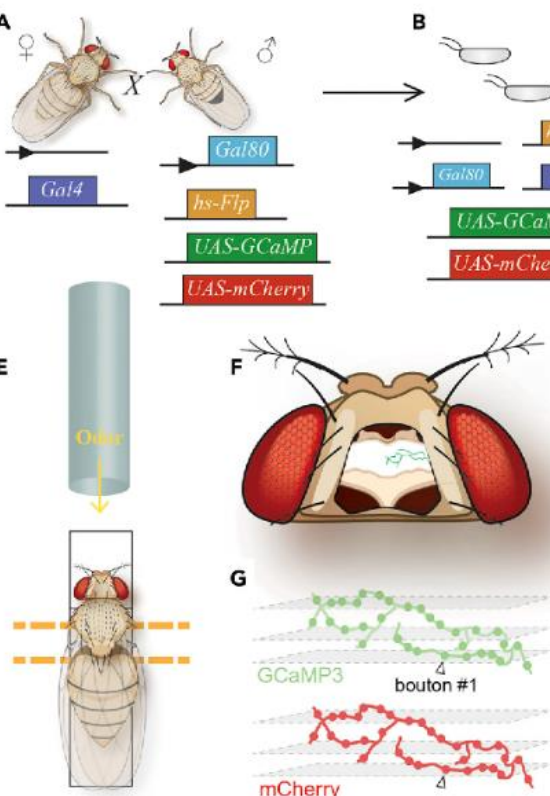

G
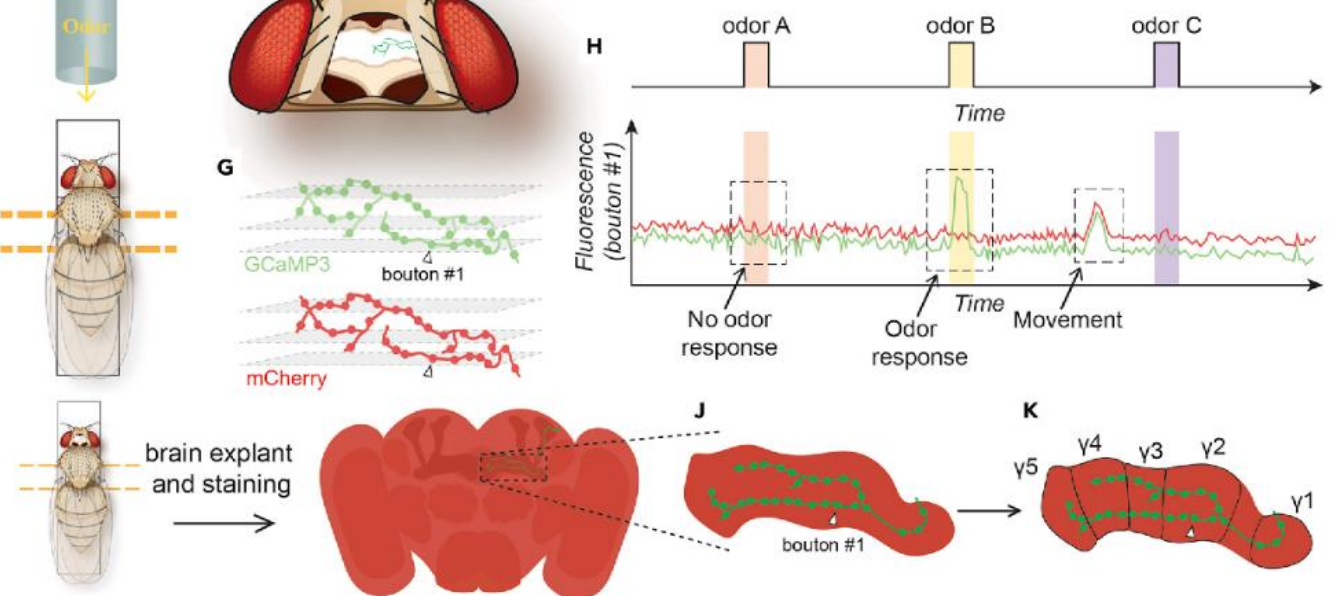

mCherry

brain explant and staining
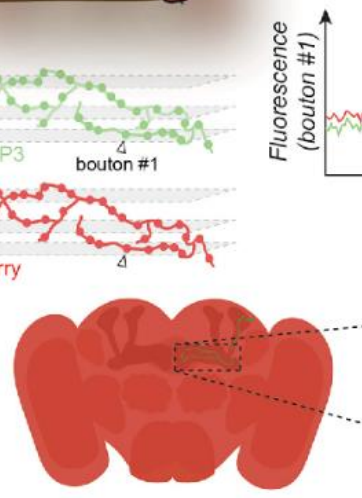

C
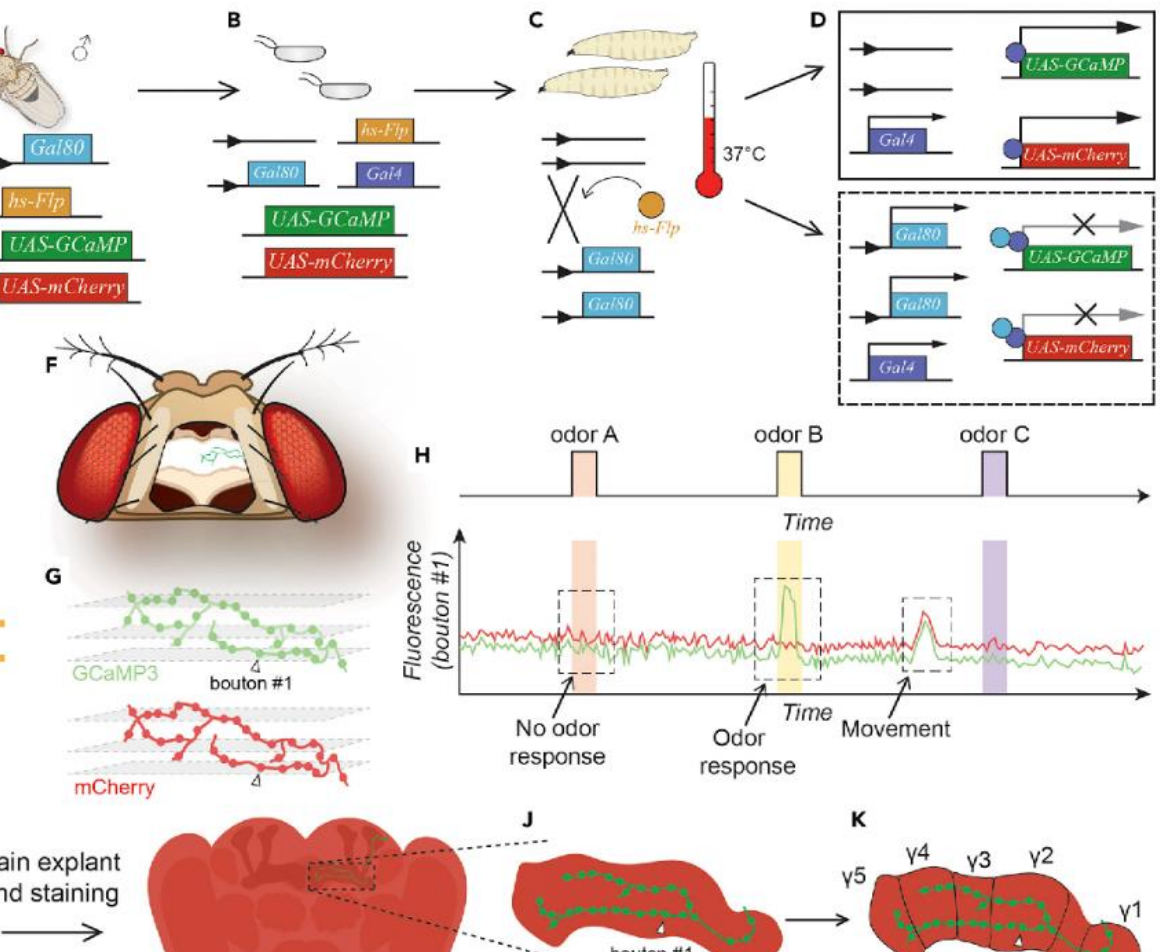

1. Schematic depiction of the generation of experimental flies and data acquisition

(A) Parental fly lines that were crossed to generate experimental flies.

(B) F1 generation produced by the parents in (A), collected at the time of egg-laying

(C) Induction of hs-Flp activity in the larval stage ( $48 \mathrm{~h}$ after egg-laying) by exposure to increased temperature $\left(37^{\circ} \mathrm{C}\right.$ in a water bath). Induction of hs-Flpmediated recombination at flippase recognition target (FRT) sites results in stochastic flipping-out of Gal80 in daughter cells.

(D) Potential outcome of the heat-shocking protocol. Daughter cells that express Gal80 (lower panel) do not show transcription of mCherry or GCaMP due to inhibition of the Gal4 transcription factor. Daughter cells in which Gal80 was flipped out (upper panel) show expression of transgenes due to normal functioning of Gal4

(E) Functional imaging setup for the visualization of odor-evoked calcium transients in the flies generated in (A-D). A single fly is fixed in a custom-built imaging chamber, placed on top of electrical wires and in the path of an airstream such that the fly can be exposed to electric shocks and odor stimuli, respectively.

(F) The head of the fly is opened to expose the brain.

(G) During functional imaging experiments, multiple planes are measured such that odor-evoked Kenyon cell responses can be visualized throughout the neuron(s). Two channels are recorded simultaneously in these experiments - one that detects emission from GCaMP, and another that detects emission from mCherry.

(H) Schematic outline of an example odor delivery protocol (top) in which the fly is exposed to three different odors while recording changes in fluorescence over time (schematically depicted in the lower panel). Use of mCherry as an activity-independent cell marker facilitates clear discrimination between small movement artefacts and changes in intracellular calcium.

(1) Following functional imaging experiments, the brain of the fly is removed and subjected to immunohistochemical staining for the anatomical characterization of measured Kenyon cells.

(J) Bouton that were measured in the previous steps $(E-H)$ can be identified from anatomical confocal scans of the same brain.

(K) Boutons can then be mapped to the different compartments of the mushroom body $\gamma$ lobe for analysis of odor-evoked responses based on anatomical position along the axon.

20xUAS-6xmCherry, 20xUAS-GCaMP3. For the exact genotypes and original sources of the flies used in this study, see Key resources table.

3. Combine the collected female and male flies in an approximate $2: 1$ ratio in a fresh food vial and allow for mating for $2-3$ days. 
Please cite this article in press as: Hancock et al., Visualization of naive and learned odor representations using in vivo calcium imaging and immunohistochemical bouton mapping of single Drosophila m..., STAR Protocols (2020), https://doi.org/10.1016/j.xpro.2020.100210

\title{
STAR Protocols
}

\section{Protocol}

MARCM technique to generate single Kenyon cell clones

\author{
(c) Timing: $\sim 10$ days
}

To achieve the expression of the cell marker (mCherry) and calcium indicator (GCaMP3) in single Kenyon cells, a temporally precise heat-shock must be applied to induce the action of Hsp70FLP1 on FRT sites. This causes stochastic flipping-out of tubP-Gal80 and allows Gal4mediated expression of transgenes downstream of UAS sites (Lee and LuO, 2001). To more specifically target $\gamma$-type Kenyon cells, here this heat-shock is applied at $48 \mathrm{~h}$ after egg laying - the time at which these cells are born (Lee, Lee, and Luo, 1999). For a schematic depiction of this step, see Figure 1.

4. Transfer the fly cross set up in the previous step to a fresh food vial for $4 \mathrm{~h}$ to collect eggs.

5. Allow these offspring to develop for $48 \mathrm{~h}$ under normal rearing conditions in an incubator.

6. $48 \mathrm{~h}$ after the egg-laying period, transfer the food vial (containing the larval offspring) to a water bath heated to $37^{\circ} \mathrm{C}$ for $45 \mathrm{~min}$ such that the lower $3 / 4$ of the vial is submerged.

7. Remove the vial from the water bath and return to the incubator for $30 \mathrm{~min}$.

8. Repeat step 6.

9. Return the vial to the incubator and allow for development until the adult stage.

10. Collect the emerging F1 generation daily and keep in standard food vials. Experiments can be conducted on flies aged between 3 and 6 days post-eclosion.

\section{KEY RESOURCES TABLE}

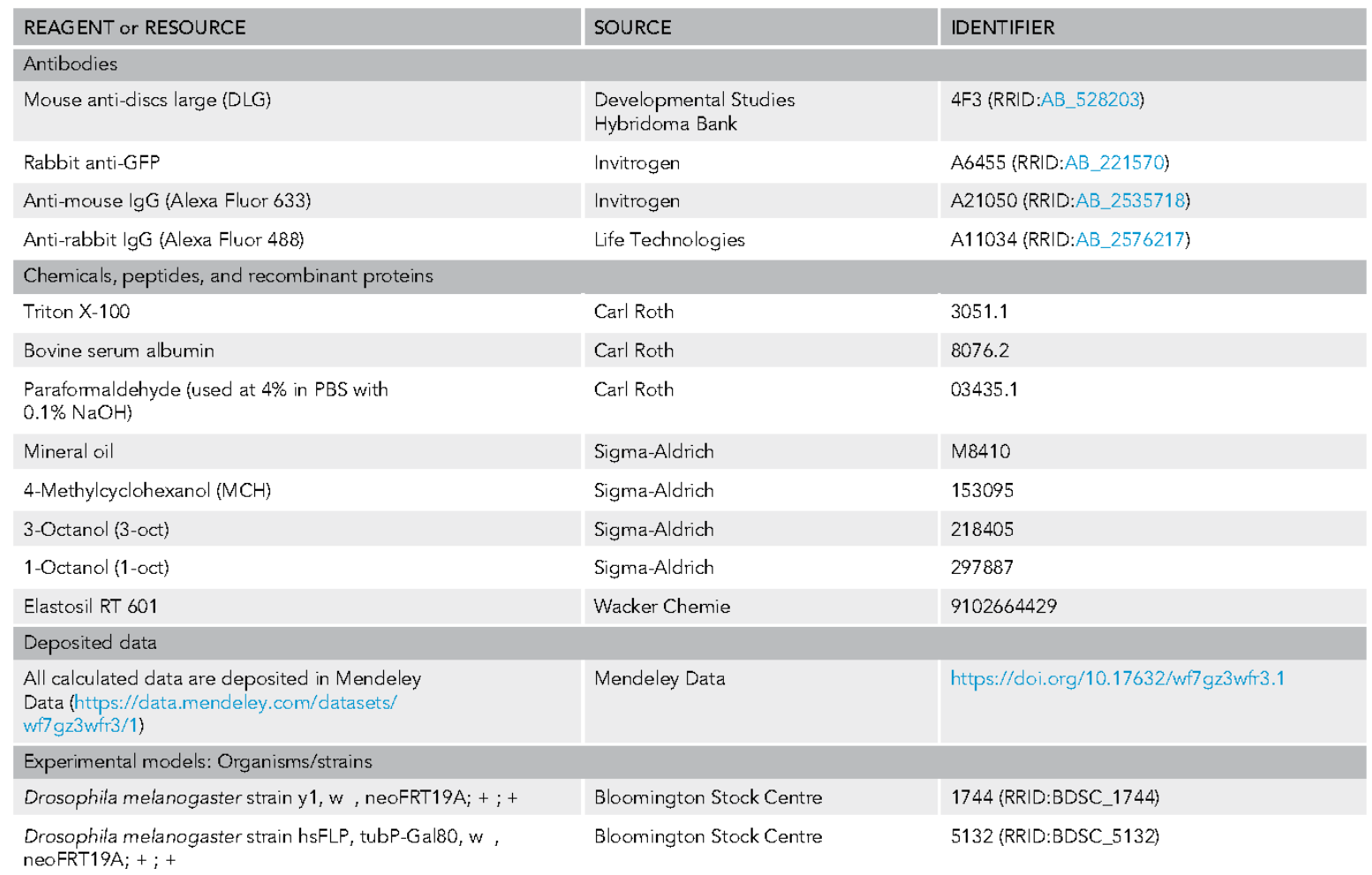


Please cite this article in press as: Hancock et al., Visualization of naive and learned odor representations using in vivo calcium imaging and immunohistochemical bouton mapping of single Drosophila m..., STAR Protocols (2020), https://doi.org/10.1016/j.xpro.2020.100210

CellPress

OPEN ACCESS

\section{STAR Protocols}

Protocol

Continued

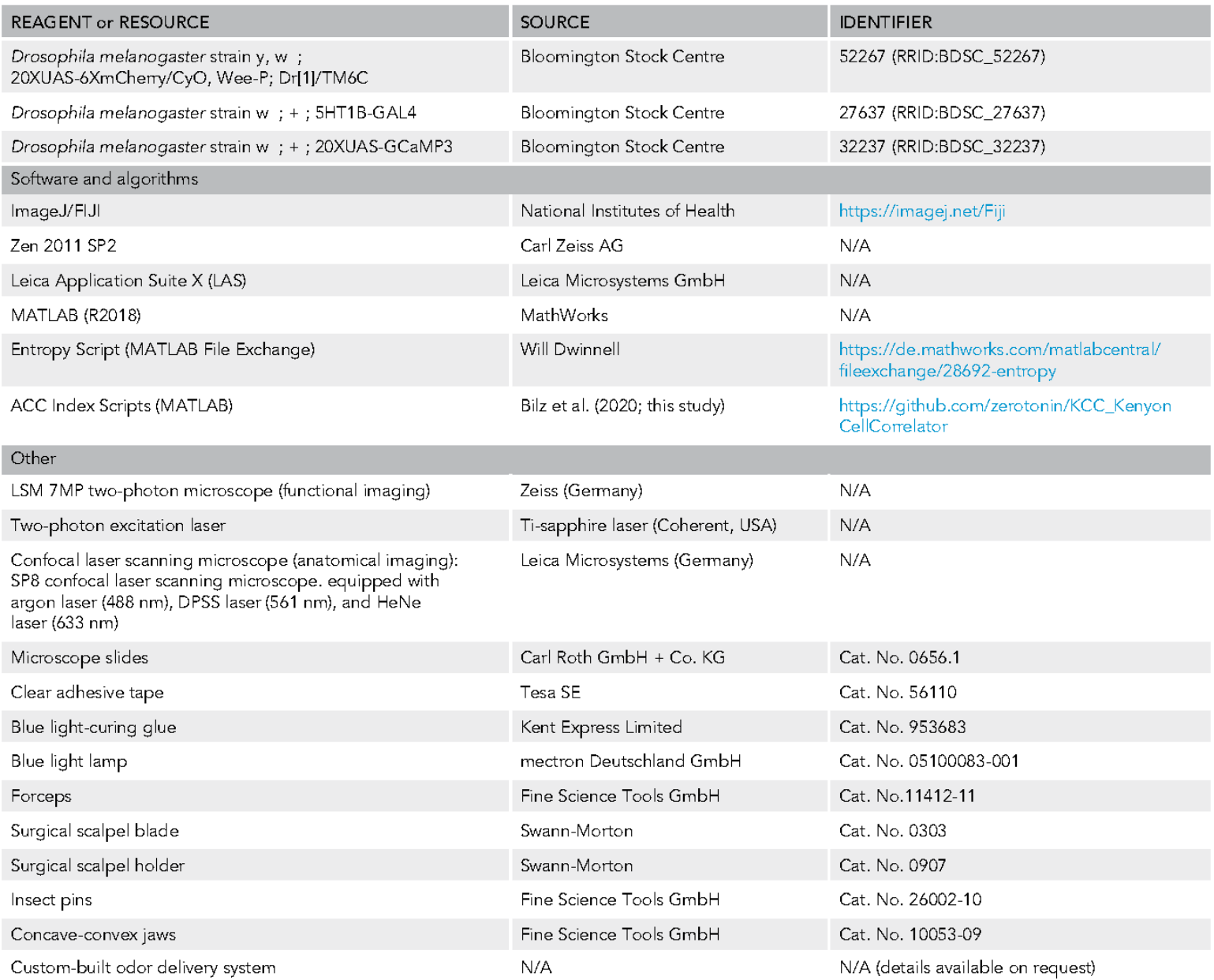

\section{MATERIALS AND EQUIPMENT}

\begin{tabular}{l|ll}
$\begin{array}{l}\text { Ringer's solution } \\
\text { Reagent }\end{array}$ & Final concentration & Amount \\
\hline $\mathrm{KCl}$ & $5 \mathrm{mM}$ & $0.3728 \mathrm{~g}$ \\
$\mathrm{NaCl}$ & $130 \mathrm{mM}$ & $7.597 \mathrm{~g}$ \\
\hline $\mathrm{MgCl}_{2} \cdot 2 \mathrm{H}_{2} \mathrm{O}$ & $2 \mathrm{mM}$ & $0.406 \mathrm{~g}$ \\
$\mathrm{CaCl}_{2}$ & $2 \mathrm{mM}$ & $0.294 \mathrm{~g}$ \\
\hline $\mathrm{HEPES}$ & $5 \mathrm{mM}$ & $1.191 \mathrm{~g}$ \\
\hline $\mathrm{Sucrose}$ & $36 \mathrm{mM}$ & $12.32 \mathrm{~g}$ \\
\hline $\mathrm{H}_{2} \mathrm{O}$ & $\mathrm{N} / \mathrm{A}$ & Up to $1 \mathrm{~L}$
\end{tabular}

Note: Ringer's solution is stored at $-20^{\circ} \mathrm{C}$ before use, then kept refrigerated at $4^{\circ} \mathrm{C}$ after defrosting for no more than 3 weeks. The solution is adjusted to $\mathrm{pH} 7.3 \mathrm{using} \mathrm{HCl}$ or $\mathrm{NaOH}$. 
Please cite this article in press as: Hancock et al., Visualization of naive and learned odor representations using in vivo calcium imaging and immunohistochemical bouton mapping of single Drosophita m..., STAR Protocols (2020), https://doi.org/10.1016/j.xpro.2020.100210

\section{STAR Protocols}

\section{Protocol}

Phosphate buffered saline (PBS) solution

\begin{tabular}{l|l|l}
\hline Reagent & Final concentration & Amount \\
\hline $\mathrm{NaH}_{2} \mathrm{PO}_{4}$ & $15 \mathrm{mM}$ & $2.07 \mathrm{~g}$ \\
$\mathrm{NaCl}$ & $100 \mathrm{mM}$ & $5.84 \mathrm{~g}$ \\
\hline $\mathrm{Na}_{2} \mathrm{HPO}_{4}$ & $85 \mathrm{mM}$ & $12.06 \mathrm{~g}$ \\
\hline $\mathrm{H}_{2} \mathrm{O}$ & $\mathrm{N} / \mathrm{A}$ & Up to $1 \mathrm{~L}$
\end{tabular}

Note: PBS solution is stored at $4^{\circ} \mathrm{C}$ for no more than 2 months.

Washing solution

\begin{tabular}{|l|l|l|}
\hline Reagent & Final concentration & Amount \\
\hline Triton X-100 & $0.6 \%$ & $600 \mu \mathrm{L}$ \\
\hline PBS & N/A & $99.4 \mathrm{~mL}$ \\
\hline Total & N/A & $100 \mathrm{~mL}$ \\
\hline
\end{tabular}

Note: Washing solution is stored at $4^{\circ} \mathrm{C}$ for no more than 4 weeks.

Blocking solution

\begin{tabular}{|l|l|l|}
\hline Reagent & Final concentration & Amount \\
\hline Bovine serum albumin & $2 \%$ & $0.2 \mathrm{~g}$ \\
\hline Washing solution & N/A & $10 \mathrm{~mL}$ \\
\hline
\end{tabular}

Note: Blocking solution is stored at $4^{\circ} \mathrm{C}$ for no more than 4 weeks.

\section{STEP-BY-STEP METHOD DETAILS}

Preparation of fly for imaging

\section{(1) Timing: $15 \mathrm{~min}$}

In this step, transgenic flies generated in the previous steps are prepared for functional imaging of odor-evoked calcium transients. For an in-depth description of these steps, also see Hancock et al. (2019). Photographs of the imaging chamber used in this study can be seen in Figure 2.

1. Select a single transgenic female fly generated in the previous steps and place in an empty plastic vial on ice for $5 \mathrm{~min}$ to anaesthetize.

2. Fix the fly in a custom-built imaging chamber using clear adhesive tape. The chamber should comprise of a narrow channel (with approximate width of $1 \mathrm{~mm}$ ) into which the fly can be placed, ventral side down, with the head supported and stabilized to reduce movement. Placed in front of the head should be a channel through which an air stream can be delivered during the experiment. Under the fly should be two parallel electrical wires through which electric shocks can be delivered to the thorax during the training phase of the protocol.

3. Cut a hole in the adhesive tape using a surgical scalpel blade to expose only the head of the fly and keeping the rest of the body covered.

4. Using blue light-curing glue, fix the head on the back and sides to limit its movement. In this study, an insect pin held by concave-convex jaws was used to manipulate the glue around the head. Leave the dorsal surface of the head free for opening. Ensure there are no gaps through which the Ringer's solution (applied in the next step) could leak. 


\section{CellPress \\ OPEN ACCESS}

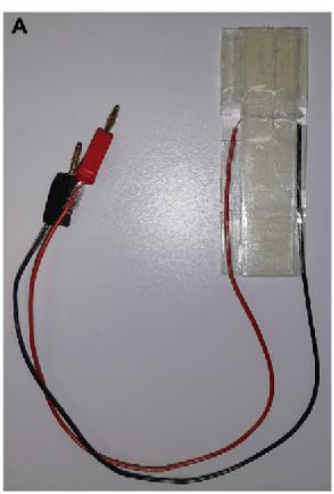

\section{STAR Protocols}

Protocol

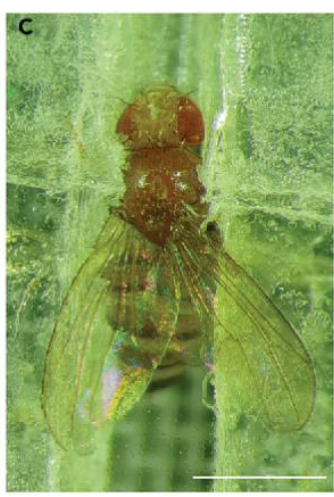

Figure 2. Custom-built fly chamber used for in vivo functional imaging experiments

(A) The fly chamber is formed of a microscope slide, plastic mesh, and clear adhesive tape and is fitted with two electrical wires that run parallel through the middle.

(B) A small hole is cut in the adhesive tape, leaving a platform to the front on which the head of the fly is later placed.

(C) A fly fixed in the chamber.

(D) The head of the fly glued in position and opened to reveal the brain.

Scale bars in $(B)$ and $(C), 1 \mathrm{~mm}$; in (D), $0.5 \mathrm{~mm}$.

5. Apply a drop of room temperature $\left(19^{\circ} \mathrm{C}-21^{\circ} \mathrm{C}\right)$ Ringer's solution on the exposed head cuticle and open the head capsule using a fine bladed knife.

6. Using fine forceps, remove the cuticle and any excess tissue that lies on top of the brain.

In vivo imaging of odor-evoked calcium transients

\section{(c) Timing: $30 \mathrm{~min}$ to $1 \mathrm{~h}$}

In this step, the flies prepared previously are imaged using two-photon microscopy to visualize odorevoked calcium transients in single Kenyon cells before, during, and after an olfactory associative conditioning protocol conducted in vivo under the microscope. Photographs of the setup used in this study can be seen in Figure 3, and schematic summary of the imaging protocol can be seen in Figures $1 \mathrm{E}-1 \mathrm{H}$.

7. Prepare the odor mixtures. In this study, MCH, 3-octanol, and 1-octanol were used at concentrations of 1:750,1:500, and 1:400, respectively, in mineral oil. Install odors in the odor delivery system.

8. Place the prepared fly under a microscope equipped for two-photon imaging. For details of the setup used in this study, see Figures 2 and 3 and the Key resources table.

9. Illuminate the preparation using a red filter set. Through the eyepieces, observe the fly brain for expression of mCherry in Kenyon cells.

$\triangle C R I T I C A L:$ due to the stochastic nature of the MARCM expression system, a variety of outcomes are possible - ranging from single cell expression to expression in dozens of cells. In order to later identify single cells, only flies in which a maximum of 3 Kenyon cells per hemisphere are labelled can feasibly be utilized in these experiments.

10. If the correct number of cells are labelled, position the fly head in the center of the field of view for imaging, ensuring the odor delivery system is in place and the electrical wires have been connected to a power source.

$\triangle C R I T I C A L:$ due to the sensitivity of detector systems used in this setup, limit external light sources as much as possible. 


\section{STAR Protocols}

\section{Protocol}

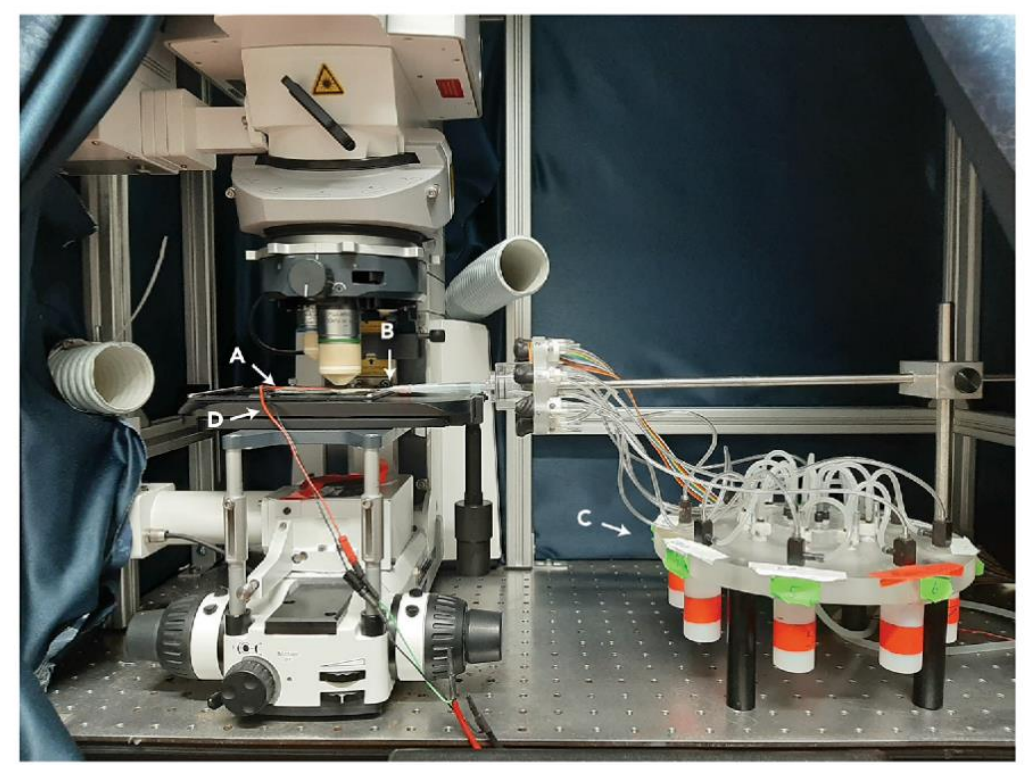

Figure 3. Microscope setup for in vivo functional imaging

The fly chamber (A) shown in Figure 2 is placed under the microscope objective and is connected to electrical wires (D) and a needle (B) that connects the chamber to the odor delivery system (C). The odor delivery machine holds eight different odor bottles, from which odors are carried by a continuous airstream by the timely controlled opening and closing of valves at their opening.

11. Start the microscope control software and initialize image acquisition settings. To visualize both red and green fluorophores, an excitation wavelength of $940 \mathrm{~nm}$ and a customized filter set with a $605 \mathrm{~nm}$ beamsplitter and two bandpass filters of $\sim 620-680 \mathrm{~nm}$ for mCherry detection, and $\sim 500-550 \mathrm{~nm}$ for GFP detection is recommended. For specific software and equipment used in this study, see Key Resources Table.

12. Initiate image acquisition and locate the labelled Kenyon cells. Crop and rotate the acquisition region such that the Kenyon cells are positioned in the center of the field of view.

13. Through multiple z-planes, run a synchronized image acquisition and odor presentation sequence whereby, in each imaging plane, all of the odors and mineral oil are presented to the fly via air stream. Save these image sequences for later analysis.

Note: $\ln$ this study, the airstream flow rate was $1 \mathrm{~mL} / \mathrm{s}$.

Note: Dependent on the number of cells and their anatomical complexity, the number of imaging planes required to capture maximal axonal branches can vary. In this study, generally between 4 and 7 planes were imaged per brain.

Note: In this study, a framerate of $4 \mathrm{~Hz}$ was used.

14. Run the associative conditioning protocol. In this protocol, present one of the odors continuously for $60 \mathrm{~s}$ with overlapping pulsing electric shocks (this is now the CS+ odor). After a $60 \mathrm{~s}$ break, present a second of the odors for $60 \mathrm{~s}$ with no electric shocks (now the CS- odor). For control groups, either omit the electric shock (CS-only control) or deliver the electric shock $2 \mathrm{~min}$ before the onset of the first odor (unpaired control).

15. For post-training data, repeat the odor presentation protocol as in step 13. Ensure that measurements are conducted in the same imaging planes as before the conditioning, so that odor-evoked responses can be compared in the same boutons. 
16. When all planes have been measured, capture a z-stack which covers the entire depth of the brain to later refer to when identifying and mapping cells/boutons.

17. Remove the imaging chamber from the microscope and, with the fly intact, proceed to the next section.

Brain explant and immunohistochemistry

(1) Timing: $\sim 2.5$ days

In this step, the brain of the fly imaged in the previous step is removed for immunohistochemical staining. This will facilitate later confocal microscopic scanning of the brain to produce high resolution images from which the functionally imaged Kenyon cells can be fully anatomically characterized.

18. Carefully remove the fly from the imaging chamber and transfer to a dissection dish containing a black-dyed silicon mixture (Elastosil-based, see Key Resources Table).

19. Fix the fly in place, ventral side up, using insect pins through the thorax and abdomen and cover with 2-3 drops of ice-cold Ringer's solution.

20. Using fine forceps, carefully remove the proboscis.

21. Pull apart the two sides of the head capsule until they are completely removed from the brain. Clear away the residual tissues from the brain.

22. Detach the brain from the thorax, leaving behind the ventral nerve cord.

23. Place the brain in fixative solution (4\% paraformaldehyde in PBS) for $2 \mathrm{~h}$ on a shaker at $4{ }^{\circ} \mathrm{C}$.

24. Wash the brain 3 times for 20 min each in PBS-T washing solution (PBS with $0.6 \%$ Triton X-100) at room temperature $\left(19^{\circ} \mathrm{C}-21^{\circ} \mathrm{C}\right)$ on a shaker.

25. Place the brain in blocking solution ( $2 \%$ bovine serum albumin in PBS-T) for $2 \mathrm{~h}$ on a shaker at room temperature $\left(19^{\circ} \mathrm{C}-21^{\circ} \mathrm{C}\right)$.

26. Transfer the brain to a dish containing a primary antibody mix containing antibodies against GFP (which will bind GCaMP in Kenyon cells) and discs-large (DLG; which will bind the pan-neuronal synaptic protein, discs-large) and incubate on a shaker at $4^{\circ} \mathrm{C}$ for $12-16 \mathrm{~h}$. In this study, we used rabbit anti-GFP (Invitrogen) at a concentration of 1:2000 and mouse anti-DLG (Developmental Studies Hybridoma Bank) at a concentration of 1:200 in blocking solution.

Note: $\mathrm{mCherry}$ autofluorescence is in most cases robust enough to not require additional fluorophore binding, so no mCherry antibody is used in this study.

27. Wash the brain as in step 24 .

28. Transfer the brains to a dish containing a secondary antibody mix. All secondary antibodies were used at a concentration of 1:300 in blocking solution. In this study, we used anti-rabbit Alexa Fluor 488 and anti-mouse Alexa Fluor 633. For further details about the antibodies used, see Key Resources Table. Incubate on a shaker at $4^{\circ} \mathrm{C}$ for $12-16 \mathrm{~h}$.

29. Wash the brain as in step 24 .

30. Mount the brain for confocal scanning. Inside a $13 \mathrm{~mm}$ tape ring, pipette approximately $5 \mu \mathrm{L}$ of VectaShield and carefully place the brain in the liquid, taking care to orient the brain so that it lays flat with the anterior of the brain facing upwards for easier scanning.

31. Scan the brain using a confocal laser scanning microscope. Laser and detector settings should be chosen according to the secondary antibodies used. To maximize image quality, use an immersion lens and a frame size of at least $1024 \times 1024$ pixels. Brains were scanned sequentially, such that GFP and DLG channels were captured simultaneously, followed by an additional scan for mCherry. For the exact hardware and software used in this study, see Key Resources Table.

Functional imaging analysis

(c) Timing: $\sim 1-1.5 \mathrm{~h}$ 


\section{STAR Protocols}

In this step, the images acquired during the functional calcium imaging step are processed and analyzed to quantify odor-evoked activity in individual synaptic boutons.

32. Install and open an image processing program. In this study, Image J was used for all image processing steps.

33. Either individually or using a custom-written batch-processor, correct each image series for any movement in the $\mathrm{X}$ and $\mathrm{Y}$ dimensions using an image registration plug-in such as TurboReg (Thévenaz et al., 1998).

34. In each imaging plane, pre- and post-training, mark regions of interest (ROIs) encompassing individual synaptic boutons along the Kenyon cell axons. Boutons can be identified by their spherical shape and their greater size relative to the axonal diameter.

$\triangle$ CRITICAL: the number of identifiable boutons for a given Kenyon cell can vary widely, in this study ranging from 29-158 boutons. To investigate the effects of training on odor representations, only boutons that are identifiable in both the pre- and post-training recordings can be used in this analysis.

35. For each bouton, use the "Multi Measure" function in Image $J$ to extract fluorescence intensity values over time for both the green (GCaMP) and red (mCherry) channels of each recording.

36. From the raw fluorescence values, calculate the $\Delta F / F_{0}$ values for each channel.

a) First, generate the $F_{0}$ value by calculating the average fluorescence values from the $5 \mathrm{~s}$ preceding odor onset.

b) Second, generate the $\Delta F$ values by subtracting the $F_{0}$ value from the raw fluorescence.

c) Finally, divide the $\Delta F$ value at each frame by $F_{0}$ to generate a $\Delta F / F_{0}$ trace over time.

37. To temporally filter the $\Delta F / F_{0}$ trace, apply a 3-frame moving average through the time series.

38. To reduce the presence of $\Delta F / F_{0}$ fluctuations that are due to slight movements in the preparation rather than calcium fluctuations, subtract the $\mathrm{mCherry} \Delta \mathrm{F} / \mathrm{F}_{0}$ from the GCaMP $\Delta \mathrm{F} / \mathrm{F}_{\mathrm{O}}$.

Kenyon cell anatomy and bouton mapping

\section{(c) Timing: $1-2 \mathrm{~h}$}

In this final image analysis step, boutons that have been functionally analyzed are allocated to a Kenyon cell (in the case of a brain in which multiple cells were labelled) and assigned to the correct compartment of the mushroom body $\gamma$-lobe. This facilitates the analysis of bouton activity in the context of their anatomical position along the Kenyon cell axon. A schematic depiction of this process can be seen in Figures $1 \mathrm{~J}$ and $1 \mathrm{~K}$.

39. In Image J, open the confocal image stacks of the immunostained brain.

40. Using the mCherry and GFP image stacks, track the Kenyon cell(s) through the image stacks and generate a 3-dimensional projection view of the Kenyon cell(s). This projection will aid in the translation of the bouton positions from the functional imaging view (dorsal) to the anatomical imaging view (anterior).

41. If necessary, separate the distinct Kenyon cells to form separate projections for each cell.

42. Using the z-stack generated during functional imaging, map the identified boutons onto the anatomical images. Boutons for which positions cannot be translated should not be analyzed.

43. Using the anti-DLG neuropil staining, identify the 5 compartments of the mushroom body $\gamma$ lobe, and note the compartment location of each of the measured boutons.

\section{Calculating the amplitude corrected correlation (ACC)}

(c) Timing: $20 \mathrm{~min}$ 
Please cite this article in press as: Hancock et al., Visualization of naive and learned odor representations using in vivo calcium imaging and immunohistochemical bouton mapping of single Drosophila m..., STAR Protocols (2020), https://doi.org/10.1016/j.xpro.2020.100210

The overall aim of the analysis described in this step is to quantify single bouton responses before and after learning. This is achieved through two different analysis protocols - the Bouton Response Class analysis (BRC) and the Amplitude Corrected Correlation (ACC) (see Figure 4). Both protocols analyze the bouton responses, with regard to the individualization of each bouton, which is in contrast to the canonical approach, in which the comprehensive cell response is quantified (e.g., in the form of calcium imaging or membrane potential). We developed the ACC to quantify the congruence of Kenyon cell synaptic boutons within the same cell. This congruence reflects both the temporal structure of the calcium responses and their amplitude.

The former can be directly quantified by the cross-correlation between both calcium responses. The correlation is further normalized by its auto correlation coefficients, limiting the numerical range of the cross-correlation from 0 (not correlated) to 1 (identical temporal structure).

$$
\frac{\int C_{1}^{*}(\tau) \cdot C l_{2}(t+\tau) \delta \tau}{\sqrt{\sum_{\alpha \in C_{1}} a^{2}} \cdot \sqrt{\sum_{\beta \in A C C_{2}} b^{2}}}
$$

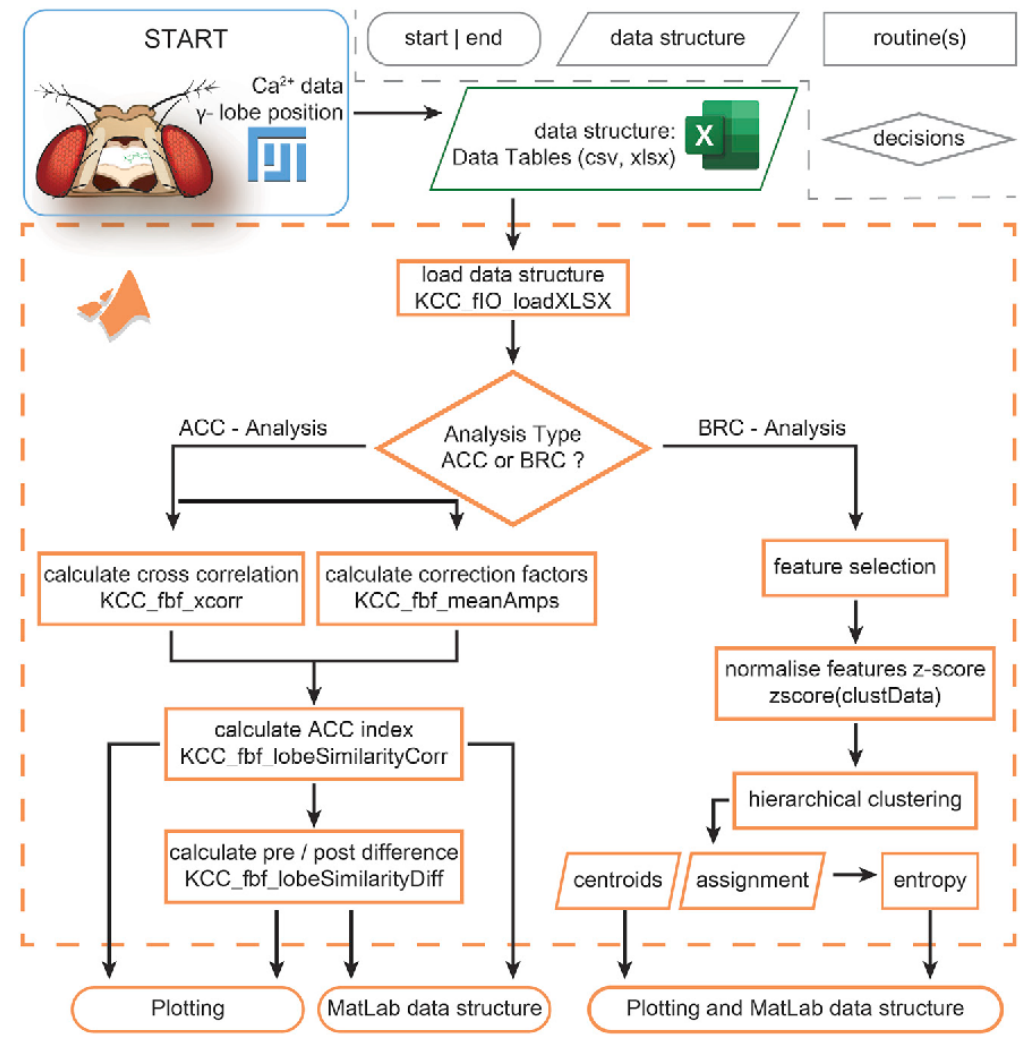

Figure 4. Workflow of the analysis pipeline

Different shapes in the flow diagram are illustrated in the upper right corner, within the dashed gray line. Rectangles with rounded corners denote the start or end of the workflow. Rectangles with sharp corners indicate routine calls. Parallelograms highlight data structures, whereas diamond shapes indicate decisions by the user. The different boxes are color coded for the different programming environments (light blue, ImageJ/Fiji; green, table calculations; orange, MATLAB). The workflow starts with the dynamics of calcium-dependent fluorescence changes in single boutons and their compartment location. These data are then transferred into $x$ lsx or csv files. The KCC loading routines then make those data available for two different analysis options: the Amplitude Corrected Correlation (ACC) index or the Bouton Response Class (BRC) analysis. The analysis pipeline ends in data structures or plots. 


\section{STAR Protocols}

\section{Protocol}

where the time-series of the calcium responses are represented by either $\mathrm{Cl}_{1}$ or $\mathrm{Cl}_{2}$. The time-point is demarcated by $t$ and the phase lag of the correlation by $\tau$.

To address the difference in response amplitude, the Michelson contrast of the two amplitudes is calculated as: $1-\left|A_{1}-A_{2} / A_{1}+A_{2}\right|$, where $A_{x}$ is the respective maximum amplitude of each calcium response. Thereby, the contrast value becomes 1 if there is no difference between both responses and approaches 0 if they are very dissimilar.

The resulting $\mathrm{ACC}$ is sensitive to congruent responses, meaning that it correctly detects similar response pairs. However, traces that lack any response will also render an ACC close to 1. To remedy this, a third term is introduced that calculates the mean response of two boutons, normalized by the maximum response of the neuron:

$$
\frac{A_{1}+A_{2} / 2}{\max \left(A_{1 \rightarrow n}\right)}
$$

$A_{1}$ and $A_{2}$ are again the maximum amplitude of the responses and $n$ represents the entirety of all responses. Thereby, flat responses are set to 0 , while robust responses will render values close to 1 .

Now each of the three measures (cross correlation, amplitude contrast, and normalized mean amplitude) are within the limits of 0 to 1 and can be multiplied to derive a sensitive and specific measure to quantify the congruence in the responses of two synaptic boutons as follows:

$$
A C C=\frac{\int C_{1}^{*}(\tau) C_{2}(t+\tau) \delta \tau}{\sqrt{\sum_{\alpha \in C_{1}} a^{2}} \cdot \sqrt{\sum_{\beta \in A C_{2}} b^{2}}} \cdot \frac{\frac{A_{1}+A_{2}}{2}}{\max \left(A_{1 \rightarrow n}\right)} \cdot\left(1-\frac{A_{1}-A_{2}}{A_{1}+A_{2}}\right) .
$$

For the MATLAB (MathWorks, USA) implementation of the aforementioned formulas, as used in this study, see Key Resources Table. In the following steps, an example function call is given that can be tested with the data deposited at https://data.mendeley.com/datasets/wf7gz3wf3/1.

44. Download the scripts from https://github.com/zerotonin/KCC_KenyonCellCorrelator/ and the sample data from https://data.mendeley.com/datasets/wf $7 \mathrm{gz} 3 \mathrm{wfr} 3 / 1$. For these scripts to run smoothly, data files must follow a strict format, as described below.

a) Response data for each cell is saved into one xlsx file. The file consists of two sheets in which sheet 1 contains the pretraining responses and sheet 2 the post-training responses.

Each sheet includes 4 tables of $m$ rows and $n$ columns. The columns hold the information for each identified bouton and each row reflects the sample time. The first row of the sheet shows in which $\gamma$-lobe compartment the bouton is situated. The first column identifies which odor was used for stimulation.

45. In this step, an $x \mid s x$ file is read and transcribed it into a 4-dimensional matrix. The input variables are defined as follows: fpos is a string with the file position of your $x \mid s x$ file. samples describe the length of each recording. gap holds the amount of empty rows between different odor presentations in the $x \mid s \times$ file. The resulting variable is the 4 dimensional data $m \times n \times p \times 2$ matrix, where $m$ is the number of frames recorded, $n$ the number of boutons, $p$ the number of presented odors, and the fourth dimension holds the pre-/post-training condition. The second return variable is a vector of $n$-length with the $\gamma$-lobe compartment identification (i.e., $\gamma 2, \gamma 3, \gamma 4$, or $\gamma 5$ ).

$\% \%$ load file

$\%$ set path to your $x \mid s x$ file

fPos $=$ '/path2/Controls/A_170320_3003_0304_fly1_cell1_pre_post.xlsx'; samples $=85$;

$\%$ number of frames acquired during $\mathrm{Ca} 2+$ imaginggap $=3$;

$\%$ empty rows between each of the odor presentations 
\% function call to read in xisx example data file

$[$ data,ylobesIDX] $=$ KCC_flO_loadXLSX(fPos, samples,gap);

Optional: If data are already in MATLAB format, steps 44 and 45 can be omitted. In this case, the data should be arranged in a 4 dimensional matrix $(m \times n \times p \times 2)$ where $m$ is the number of frames recorded during the experiment, $\mathrm{n}$ is the number of boutons identified in the cell and $P$ is the number of presented odors. The fourth dimension represents the pretraining recording and then the post-training recording.

46. In the next step, the cross-correlation for all boutons against all other boutons is calculated. To do so, use the data variable created in the last step and define the following inputs: corrWin is a 2 entry integer vector holding the first and the last frame of the odor presentation. $f p s$ is the frame rate of the recording. maxPhaseShift is the maximum temporal deviation between two calcium traces allowed during the cross-correlation analysis. The function call "KCC_fbf_xcorr" will result in another $4 \mathrm{D}$ matrix $(\mathrm{m} \times \mathrm{m} \times 4 \times 2)$ holding the correlation coefficients normalized by their auto correlation such that these values will be scaled from 0 (no correlation) to 1 (identity). Here, $m$ is the number of boutons, the third dimension holds odor identity, and the fourth dimension is pre-/post-training. The diagonal of the $m \times m$ slides of the cross-correlation matrix are $\mathrm{NaN}$ (not a number) values, as these represent identity and are therefore not explicitly calculated. Function call for this step:

$\% \%$ calculate the cross correlation between all boutons\%data is the output variable of $K C C_{-}$flO_loadXLSXCorrWin $=[413] ; \%$ correlation window

$\mathrm{fps}=4 ; \%$ frames per second

maxPhaseShift $=5 ; \%$ maximum of temporal deviation between both boutons

\%function call to calculate cross correlation corrMat $=$ KCC_fbf_xcorr(data,corrWin,fps, maxPhaseShift);

47. Next, prepare the amplitude data using the $\mathrm{KCC}_{-}$fbf_meanAmps function to then combine everything in Step 48 . This function call gets inputs from the previously run functions, and results in 3 new variables: dataM is a $\mathrm{m} \times 4 \times 4 \times 2$ matrix holding the median response for all boutons of each $\gamma$-lobe compartment, where $m$ is the number of frames recorded. The second dimension is the four $\gamma$-lobe compartments, the third represents the four odors, and the fourth is the pre- $/$ post-training condition. amps is a $4 \times 4 \times 2$ matrix in which the first dimension represents the $\gamma$ lobe compartment, the second dimension the odor, and the third the pre-/post-training condition.

$\% \%$ calculate the correction factors

$\%$ calculate the mean response

$[$ dataM, amps,ampsN] = KCC_fbf_meanAmps(data,ylobesIDX,corrWin,fps);

48. Finally, to calculate the $\mathrm{ACC}$ index, run the $\mathrm{KCC}$ fbf_lobeSimilarityCorr function. The resulting variables are the $A C C$ and lobesCorr. lobesCorr is a $m \times m \times 4 \times 2$ matrix, where $m$ is the number of $\gamma$-lobe compartments. Each entry is the median of all bouton-to-bouton correlations specific for a compartment, an odor, and the pre-/post-training condition. The odors are represented in the third dimension and the pre-/post-training condition is represented in the fourth dimension. The ACC is identical to the lobesCorr variable, but is multiplied with the amplitude contrast and relative amplitude strength, as below:

$\%$ calculate ACC index

$[A C C$, lobesCorr $]=$ KCC_fbf_lobeSimilarityCorr(corrMat,ylobesIDX, amps, ampsN):

49. To calculate the pre- to post-training differences in ACC indices, use the function "KCC_fbf_lobeSimilarityDiff", which will carry out a simple subtraction. The resulting 3D matrix contains the difference in $A C C$ as a $m \times m \times 4$ matrix where $m$ is the number of $\gamma$-lobe compartments and the third dimension represents the four different odors presented in the experiment.

$\% \%$ calculating the pre post difference of the ACC accDiff = KCC_fbf_lobeSimilarityDiff(ACC); 


\section{STAR Protocols}

Optional: If only pre- or post-training data are of interest (and not the comparison between them), step 49 can be omitted.

50. Save the data into a MATLAB data file:

$\%$ save result

$\%$ set file position where data should be saved

savePos $=$ '/folder $2 /$ saveDestination.mat';

$\%$ saving to disk

save(savePos,'ACC','accDiff','corrMat','dataM','data',...

'ylobesIDX', 'amps','ampsN','lobesCorr');

51. Through steps 49 and 50 , the primary analysis for the experiment is completed. To plot the results of these steps, use the following functions:

$\% \%$ plotting

$\%$ bouton-to-bouton cross-correlation

KCC_plot_plotBoutonCorrelation(1,corrMat,ylobesIDX);

$\%$ mean bouton responses by compartment (see Bilz et al. (2020), Figure 3A)

KCC_plot_deltaFbyFmeans $([2,3]$,data, dataM,ylobesIDX);

$\%$ ACC indices (see Bilz et al. (2020), Figure 6B, left and center plots)

KCC_plot_plotLobeSimilarityCorrelation([4 5], lobesCorr,ACC);

$\%$ calculate pre- to post-training ACC differences (see Bilz et al. (2020), Figure 6B rightmost plots)

KCC_plot_plotLobeSimDiff(6,accDiff);

Note: The full MATLAB script used in this study is available by following the link in the Key Resources Table.

\section{ACC analysis}

\section{(c) Timing: $20 \mathrm{~min}$}

In the previous step, single synaptic bouton responses were quantified in the form of the ACC. Now, these results are used to acquire information about the responses of all boutons of a single neuron.

In this step, the resulting data from the ACC calculation is combined to draw conclusions about the congruity of bouton responses and, more specifically, how this is modulated through the associative conditioning paradigm applied during the experiment. To this end, it is necessary to build so-called "collections". That is, compose a collection of a subset of the data grouped according to the different experimental conditions (e.g., the different training conditions used in this study). This collection can then be analyzed further, and the results can be seen in Bilz et al. (2020), Figure 6.

52. Compose the data collection by choosing the datasets that fit all criteria. The data collection variable should be the compiled dataM variables for the specific odor presentation (e.g., $\mathrm{CS}+, \mathrm{CS}-, \mathrm{CS}$-only). This would in theory result in a 5 dimensional $\mathrm{m} \times \mathrm{m} \times 1 \times 2 \times \mathrm{p}$ matrix, where $\mathrm{m}$ represents the number of $\gamma$-lobe compartments, the third dimension is the chosen stimulus odor, the fourth dimension represents the pre-/post-training condition, and $p$ represents the different cells. As the third dimension now became a singleton dimension we can eliminate it by using the squeeze function in MATLAB. The $5 \mathrm{D}$ matrix $(\mathrm{m} \times \mathrm{m} \times 1 \times 2 \times p)$ thereby becomes $4 \mathrm{D}(\mathrm{m} \times \mathrm{m} \times 2 \times \mathrm{p})$, and here we call this variable "collection".

$\% \%$ reducing the collection dimensionality collection $=$ squeeze $($ collection $5 \mathrm{Dl})$;

53. Using the collection variable, calculate the median pretraining condition as follows:

$\% \%$ calculating median pretraining ACC indices

medPre $=$ median $($ collection $(:, ; 1, ;), 4)$ 
54. Now, the difference between the pre- and post-training conditions can be calculated using the bsxfun function and, again, using the squeeze function, this matrix can be reduced from four dimensions $(m \times m \times 1 \times p)$ to three $(m \times m \times p)$, where $m$ is the number of $\gamma$-lobe compartments and $p$ is the number of cells.

$\% \%$ calculating pre-/post-training difference

diffData = bsxfun(@minus, collection $(:, 2,2$, , medPre);

diffData = squeeze(diffData);

55. In this step, the median response difference between pre- and post-training is determined and tested for statistical significance. In this study, we used a non-parametric confidence interval (Cl). Using this test, we ask whether there is a statistically significant change (either increase of decrease) in the ACC indices before and after the different training protocols implemented in the experiment. The function definition used in this study is largely based on standard functions of MATLAB and is used as below:

$\% \%$ calculating the median differences and the corresponding $95 \%$ confidence interval medDiff = median $($ diffData, 3$)$;

$\%$ lo represents the lower confidence interval and hi the higher confidence interval $[$ lo, hi $]=$ confintND(diffData,3);

If lo and hi are both below zero, there is $95 \%$ confidence that there is a decrease in the bouton response congruity. If both are above zero there is $95 \%$ confidence that there is an increase in the bouton response congruity. In both cases, the null hypothesis that the congruity does not change from pre- to post-training condition can be rejected with a $5 \%$ reversal probability.

\section{Bouton response classes}

\section{() Timing: $30 \mathrm{~min}$}

In this step, bouton responses are categorized into distinct classes, so-called Bouton Response Classes (BRCs). Each class then represents a distinguishable response signal. Thereby we can reduce the complexity of the responses from a continuous signal with complex response properties (amplitude, latency, etc.) to a single class. This can then be used to examine which classes are prevalent under different conditions (e.g., pretraining, CS+ post-training, CS- post-training, etc.).

The calculation of Bouton Response Classes (BRC) is an augmenting analysis to the ACC. Where the $A C C$ aims to check if the response of the boutons becomes more or less similar in respect to the training, the BRC approach aims to describe the different response types of boutons. Using a similar collection as in the ACC analysis, we can use hierarchical agglomerative clustering to identify and analyze recurring odor response types (so-called BRCs) observed across Kenyon cell boutons.

The first step in this analysis is feature selection. Features are parameters of the data that can be quantified and are characteristic of the data set to be analyzed. In this study, we used the peak amplitude $\left(\Delta \mathrm{F} / \mathrm{F}_{0}\right)$ during the stimulus presentation and the latency to the peak (s).

Agglomerative hierarchical clustering initially treats every sample of the data set (i.e., each bouton response) as one cluster. The clustering algorithm then calculates the distances between data samples and merges them into progressively fewer, larger clusters. In this study, we used simple Euclidean distance. We also used Ward's criterion (Murtagh and Contreras, 2017) to select which samples are merged first, such that minimal within-cluster variance is prioritized.

The result of such a clustering algorithm is the assignment of each sample to one cluster, in this case assignment of each observed bouton response to a BRC. Once the BRCs are calculated, their median response, frequency of occurrence and, in this case, how these parameters change from preto post-training condition, can be calculated. 


\section{STAR Protocols}

56. Select all cells of your collection by the appropriate criteria (e.g., training condition).

57. Load the data, ylobesIDX, and fps variable for each of your cells. data is a $m \times n \times p \times 2$ matrix, where $m$ is the number of frames recorded, $n$ the number of boutons, $p$ the number of presented odors, and the fourth dimension holds the pre-/posttraining condition. ylobesIDX is a vector of $n$-length with the $\gamma$-lobe compartment identification $(\gamma 2, \gamma 3, \gamma 4$, or $\gamma 5)$. Select the odors that are of interest. $\mathrm{fps}$ is the framerate used when acquiring the calcium traces.

58. For the pretraining condition of each bouton, calculate the maximum amplitude (amp) and the latency (ampT) to that amplitude. Given that a single response is saved in the vector boutResp, this can be done as follows:

$\%$ calculate bouton response features

$\%$ find all peaks inside the stimulus presentation

[ampHeights, frames]=findpeaks(boutResp(startOfStimPresentation:endOfStimPresentation));

$\%$ find the highest peak[amp,ampT] =max(ampHeights);

$\%$ find the original frame of the peak and divide by the fps to calculate it in seconds amp $T=$ frames $(a m p T) / f p s ;$

59. Store the results from step 58 in one $m \times 2$ matrix (clustData) where $m$ is the number of all boutons involved in this collection, and the first column holds the amp values and the second column the ampT values.

$\triangle C R I T I C A L:$ it is important to be able to associate your sample consisting of these features with the cell, $\gamma$-lobe compartment, and other critical information for the later analysis.

60. As the peak and the latency are measured in different units and widely differ numerically, in this step they are normalized to a mean of zero and a standard deviation of one - a process called zscoring (Kreyszig, 1979). Z-score clustData via MATLAB's built-in zscore function.

$\% \%$-scoring clustDataZS = zscore(clustData);

61. This data can now be clustered via MATLAB's hierarchical clustering algorithms, and a dendrogram and the derivative of the clustering costs over the number of clusters can be plotted (see Bilz et al. (2020), Figure 7A). The dendrogram shows each data sample on the $x$ axis and the costs of merging on the y axis. The relationship between merger cost and cluster number is directly demonstrated by the second plot generated in this step - for which the derivative of the cost is normalized to the sum of the costs.

$\% \%$ clustering

$\%$ agglomerative hierarchical clustering with Euclidean distance and Ward's criterion

Z = linkage(clustDataZS,'ward','euclidean');

$\%$ plotting a dendrogram with as many leaves as data samples

dendrogram $(Z$, size(clustDataZS, 1$)$ )

$\%$ calculating the costs relative to number of clusters

cost $=$ flipud $(\operatorname{diff}(\mathrm{Z}(:, 3)))$;

cost $=$ cost. $/$ sum $(\cos t)$;

$\%$ plotting the costs - from 2 to length +1 , as the derivative is $n-1$

plot(2:length(cost) +1 , cost $)$

set(gca,'XScale','log')

62. Based on the two graphs generated in step 61, one can identify the optimal number of clusters to utilize based on the increasing cost relative to sample mergers. This increase identifies to the user the number of clusters that can be formed before the cost of merges - due to the sudden merging of very distinct samples - rapidly rises. We refer to this number of clusters as kMax. In this study, the kMax of the dataset was 4. By using the cluster function of MATLAB, determine the assignment of the samples to the different clusters (BRCs) in the IDX variable:

$\% \%$ get cluster assignment

IDX = cluster $\left(Z,{ }^{\prime}\right.$ maxclust', kMax $)$ 
63. Based on the IDX, calculate the mean response and the centroid of each BRC by running the following commands:

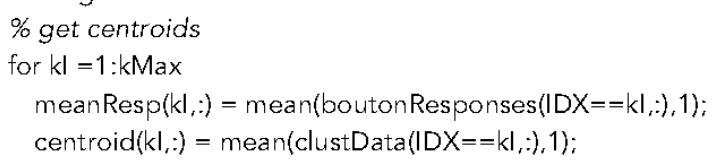

64. Combine the IDX variable with the ylobesIDX variable to calculate how often a given $B R C$ is found in a specific $\gamma$-lobe compartment. As the compartment identifiers in our case range from 2 to 5 and the BRC identifiers range from 1 to 4 , this data can be saved in a $4 \times 4$ matrix (BRC_occ):

$\% \%$ calculating the BRC occurrence per $\gamma$-lobe compartment

$\%$ go through compartment identities

for $\mathrm{yl}=2: 5$

$\%$ go through $B R C$ classes

for brcl $=1: 4$

$\%$ sum up the combined logical indices

BRC_occ $($ brcl,yl-1) = sum(ylobesIDX $==y|\& \&| D X==b r c \mid)$;

end

end

$\triangle$ CRITICAL: Make sure to keep the different data descriptors aligned. The assignment of $B R C s$ is only relevant if you can associate it with the correct cell, treatment, $\gamma$-lobe compartment, etc.

\section{Calculating bouton entropy}

\section{(c) Timing: $10 \mathrm{~min}$}

In the previous step, odor response data were transformed from numerical to categorical data by assignment into BRCs. This facilitates the application of a more direct calculation of the variance of the neuronal activity that was observed in this study.

To do this, we used the Shannon entropy (Shannon, 1949) as a measure for variance (Allaj, 2018). As entropy is measured in bits, we can directly see how many categories (BRCs, in this study) are needed to describe the responses in a $\gamma$-lobe (see Bilz et al. (2020) for more on this principle).

In this study, we used a MATLAB script created by Will Dwinnel (see Key Resources Table) implemented as below.

65. Choose a BRC IDX variable for the training condition and $\gamma$-lobe compartment of interest.

66. Run the Entropy function as below:

$\%$ calculate entropy for $\gamma$-lobe

entropyRes = Entropy $(\mathrm{DXX}(\mathrm{ylobes} \mid \mathrm{DX}==2))$;

\section{EXPECTED OUTCOMES}

Using the method described here, we were able to generate transgenic flies that showed expression of our genetically encoded calcium indicator and cell marker in 1-3 Kenyon cells with a success rate of approximately $50 \%$. The remaining flies generated showed expression in 4 or more cells, or, in some cases, no cells were labelled at all. The protocol shown here was attuned to achieve the optimal cell number for these experiments, and deviations from the heat-shock protocol outlined here would likely cause this success rate to fall. An example of a fly in which two cells were labelled and distinguished can be seen in Figure 5. 
Please cite this article in press as: Hancock et al., Visualization of naive and learned odor representations using in vivo calcium imaging and immunohistochemical bouton mapping of single Drosophila m..., STAR Protocols (2020), https://doi.org/10.1016/j.xpro.2020.100210

\section{STAR Protocols}

\section{Protocol}
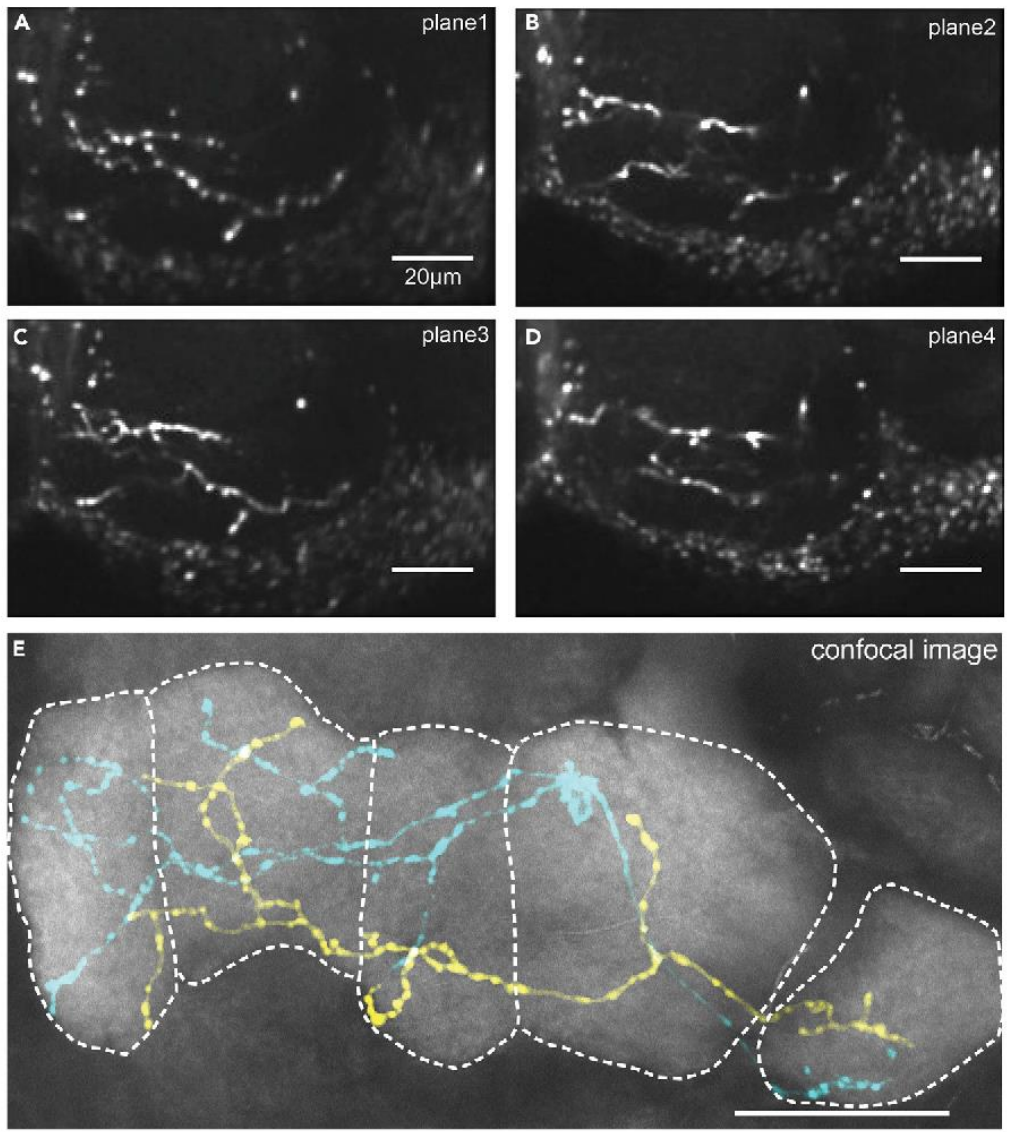

Figure 5. Example images obtained in this study

(A-D) Four example functional imaging planes show the axo nal branches of the two labelled Kenyon cells. (E) After immunohistochemistry, a confocal image series is used to disentangle the branches and identify individual cells, as well as mushroom body compartments. Here, a maximum projection is shown. All scale bars, $20 \mu \mathrm{m}$.

Odor representations in the mushroom body are encoded very sparsely, meaning for each given odor only a very small proportion of the cell population is activated. In this study, we found that approximately $8 \%, 10 \%, 4 \%$, and $2 \%$ of measured cells responded to $\mathrm{MCH}, 3$-octanol, 1 -octanol, and the diluent mineral oil, respectively (Bilz et al., 2020). This is in the range to be expected, based on previous studies (Honegger, Campbell, and Turner, 2011).

\section{QUANTIFICATION AND STATISTICAL ANALYSIS}

\section{Data inclusion/exclusion criteria}

This protocol and analysis are based on cells that exhibit responses to the olfactory stimuli used. Therefore, non-responsive cells are excluded from the analysis. Additionally, boutons must be excluded from the pre- to post-training comparison if they are not clearly identifiable in both recordings or they cannot be assigned accurately to a single cell or a mushroom body $\gamma$-lobe compartment.

Similarly, if excessive movement of the fly brain during odor response measurements causes boutons to leave the focal plane such that their fluorescent intensities could not be accurately and reliably captured throughout, those recordings must also be discarded. 


\section{LIMITATIONS}

In order to visualize calcium transients at the level of the presynaptic boutons and attribute those boutons to specific cells and mushroom body compartments, only 1-3 Kenyon cells can be imaged per fly. With the approximately $50 \%$ success rate of the MARCM technique in generating such flies and the additional limitation of only approximately $7 \%$ of Kenyon cells responding to a given odor. many trials are required in order to generate a robust sample size.

\section{TROUBLESHOOTING}

\section{Problem 1}

No (or few) odor responses are detectable during calcium imaging step (step 13).

\section{Potential solution}

If the percentage of imaged Kenyon cells that show odor-evoked responses is less than approximately $5 \%-8 \%$, ensure that the fly has not died during the preparation or imaging procedure and that the brain is still intact. Also make sure that no glue or Ringer's solution is covering the antennae and that odor delivery is not otherwise obstructed.

\section{Problem 2}

Flies die during functional imaging experiments (steps 1-17).

\section{Potential solution}

If the imaging chamber is not constructed with the correct dimensions, it is possible that the body of the fly is compressed and the fly may die either through the process of the preparation or during the experiment. Ensure the fit of the chamber is adequate to restrain the fly and limit movement, without causing injury.

Excessive humidity and/or the presence of liquid in the imaging chamber or on the thorax of the fly (e.g., due to leaks of Ringer's solution during preparation, or condensation carried over from the chilled plastic vials used for anesthesia) can lead to a burning of the fly during the electric shock presentation. Ensure the seal around the head of the fly is water-tight (using the blue light-curing glue) to avoid leaks, and take care to not transfer any liquid on the body of the fly when placing it in the imaging chamber (e.g., by ensuring the plastic vial is dry before placing the fly inside).

\section{Problem 3}

No cells are labelled (step 9)

\section{Potential solution}

Ensure that all transgenes are present in the flies generated. Also, during the heat-shock step, make sure that the vials are adequately submerged in the water bath so that larvae cannot crawl up the vial walls and partially escape the heat-shock.

\section{RESOURCE AVAILABILITY}

\section{Lead contact}

Further information and requests for resources and reagents should be directed to and will be fulfilled by the Lead Contact, André Fiala (afiala@gwdg.de).

\section{Materials availability}

This study did not generate new unique reagents.

\section{Data and code availability}

The dataset generated during this study are available on Mendeley (https://doi.org/10.17632/ wf7gz3wfr3.1) and code is deposited on GitHub (https:/github.com/zerotonin/KCC_KenyonCell Correlator). 
Please cite this article in press as: Hancock et al., Visualization of naive and learned odor representations using in vivo calcium imaging and immunohistochemical bouton mapping of single Drosophila m..., STAR Protocols (2020), https://doi.org/10.1016/j.xpro.2020.100210

STAR Protocols

Protocol

\section{ACKNOWLEDGMENTS}

We are grateful to the Bloomington Drosophila Stock Center for providing fly strains. We thank Florian Bilz for his contribution in establishing many of the procedures used in this study. We thank the Department of Applied Mathematics (University of Goettingen) for providing MATLAB licenses. This work was supported by the German Research Council (FOR 2705 and SFB 889/B4), the Volkswagen Foundation, and the Ministry for Science and Culture of Lower Saxony (ZN 3014).

\section{AUTHOR CONTRIBUTIONS}

A.F. conceived and supervised the study. C.E.H. and B.R.H.G. wrote the manuscript. C.E.H. performed imaging experiments and analysis. B.R.H.G. designed and performed the computational analyses.

\section{DECLARATION OF INTERESTS}

The authors declare no competing interests.

\section{REFERENCES}

Allaj, E. (2018). Two simple measures of variability for categorical data. J. Appl. Stat. 45, 1497-1516.

Bilz, F., Geurten, B.R.H., Hancock, C.E., Widmann, $A_{1}$, and Fiala, A. (2020). Visualization of a distributed synaptic memory code in the Drosophila brain. synaptic memory code
Neuron $106,963-976$.

Hancock, C.E., Bilz, F., and Fiala, A. (2019). In vivo optical calcium imaging of learning-induced synaptic plasticity in Drosophila melanogaster. J. Vis. Exp. 152, e60288.
Honegger, K.S., Campbell, R.A., and Turner, G.C. (2011). Cellular-resolution population imaging reveals robust sparse coding in the Drosophil mushroom body. J. Neurosci. 31,11772-11785.

Kreyszig, E. (1979). Advanced Engineering Mathematics, Fourth Edition (Wiley)

Lee, T., Lee, A, and Luo, L. (1999). Development of the Drosophila mushroom bodies: sequential generation of three distinct types of neurons from neuroblast. Development 126, 4065-4076.
Lee, T, and Luo, L. (2001). Mosaic analysis with a repressible cell marker (MARCM) for Drosophila neural development. Trends Neurosci $24,251-254$

Murtagh, F., and Contreras, P. (2017). Algorithms for hierarchical clustering an overview, II. Wiley Interdiscip. Rev. Data Min. Knowl. Discov. 7, e1219.

Thévanaz, P., Ruttiman, U.E., and Unser, M. (1998). A pyramid approach to subpixel registration based on intensity. IEEE Trans. Image Process. 7, $27-41$ 


\subsection{Discussion}

The method described here was designed and implemented to provide a novel approach to a longstanding question - that is, what form does a memory trace take in the brain? In Drosophila, the development of genetic tools that allow for the precise expression of transgenes that can facilitate anatomical and functional investigation of neural circuits has been vital to progress in this field. By expressing exogenous proteins that allow the monitoring of neuronal activity in specific cells and subjecting animals to an experimental procedure that leads to the induction of associative learning, it is possible to observe patterns of activity in the brain that coincide with learning and memory formation.

There are three primary neural populations involved in associative learning in Drosophila: the mushroom body (MB) intrinsic neurons (Kenyon cells, $\mathrm{KCS}$ ) that receive olfactory input from the antennal lobe via olfactory projection neurons; the dopaminergic neurons that convey the presence of the punishment/reward during training; and the mushroom body output neurons (MBONs) that are downstream of both other types and are implicated in encoding odour valence. Distinct dopaminergic neurons and $\mathrm{MBONs}$ innervate the $\mathrm{MB}$ lobes in a tiling manner, whereby the axons of the KCs pass through distinct compartments along the MB lobes that are exposed differential inputs and outputs. This means that the axonal projections of a single KC are likewise compartmentalised and may therefore exhibit variable degrees and directionality of plasticity dependent on compartment.

Previous optical imaging studies of the KCs have often taken one of two approaches to monitoring their activity: measuring at the cell body level, allowing for measurement of individual KCs but limiting spatial information, or measuring at the axon level where spatial information (i.e., compartment location) is available, but the dense entanglement of neurons makes identification of single cells impossible. Here, we present a technique that that combines the best of both approaches. Using the MARCM (mosaic analysis with a repressible cell marker) technique, we demonstrated a method whereby individual KCs can be functional imaged, in vivo, throughout a classical conditioning protocol. By then removing the brain and subjecting it to immunohistochemical staining and confocal microscopy, we could map the location of presynaptic structures - here referred to as synaptic boutons - dependent on the compartment they fall into along the MB lobe. Thus, this method makes it possible to analyse learning-induced odour coding in the KCs in the context of putative input and output neurons. By therein shifting the focus of analysis from cells to individual synaptic boutons as the primary unit of odour coding, this method granted more precise dissection of the mechanisms underlying this coding and thus how this distributed odour code is modified through the process of olfactory conditioning. 


\section{Visualization of a Distributed Synaptic Memory Code in the Drosophila Mushroom Body}

\subsection{Introduction and aims}

In this section, one can find the publication "Visualization of a Distributed Synaptic Memory Code in the Drosophila Brain", in which we implemented the methodology described in section 5 to investigate the neuronal mechanisms underlying the formation of an aversive olfactory memory in the mushroom body.

The neurons that form the mushroom body - the Kenyon cells (KCS) - are positioned downstream of both olfactory projection neurons and modulatory dopaminergic neurons, thus placing them at the coincidence point of the two primary sensory inputs required for the formation of olfactory associative memories. Therein, presenting a fruit fly simultaneously with both a neutral odour stimulus and an innately aversive stimulus (e.g., electric shock to the body) leads to temporally aligned inputs to the mushroom body $\mathrm{KCs}$ that triggers a series of intracellular processes that ultimately lead to plastic changes to the synapses between the KCs and their downstream partners. In particular, changes in the odour-evoked KC-to-mushroom body output neuron (MBON) drive is believed to be of particular importance for the adaptation of behaviour in response to a learned odour (see also section 4).

In vivo calcium imaging is used here to visualise changes in intracellular calcium concentration as a result of odour presentation under different learning conditions. Where previous studies have investigated KC odour coding either by visualisation of activity across large populations of KCs in the lobes (where large bundles of KC axons intertwine with one another) or activity of single KCs at the cell body layer, we here implemented a new approach (described in section 5). By driving expression of a calcium indicator in only one $\mathrm{KC}$ per fly, we were able to visualise calcium dynamics in the axonal terminals of single $\mathrm{KCs}$, the anatomy of which were fully characterised subsequent to functional imaging.

Given the spatially distinct innervation patterns of mushroom body efferent neurons along the $\gamma$-lobe (described in section 4), this provides a novel insight into how both naïve and learned odours are differentially represented along the axons of individual KCs with regard to their putative input and output circuitries. 
6.2. Manuscript - Visualization of a Distributed Synaptic Memory Code in the Drosophila Brain

Neuron, June 2020

Authors: Florian Bilz*, Bart R.H. Geurten*, Clare E. Hancock*, Annekathrin Widmann*, André Fiala

*Equally contributing first author

Contribution:

Revision of submitted manuscript resultant from previous doctoral candidate (F. Bllz), including repetition of original findings, additional control experiments, and the revision of figures and text per reviewer comments/recommendations together with supervisor and co-authors.

Contributed figures can be found in Appendix (12.2.). 


\section{Neuron}

\section{Visualization of a Distributed Synaptic Memory Code in the Drosophila Brain}

\section{Graphical Abstract}

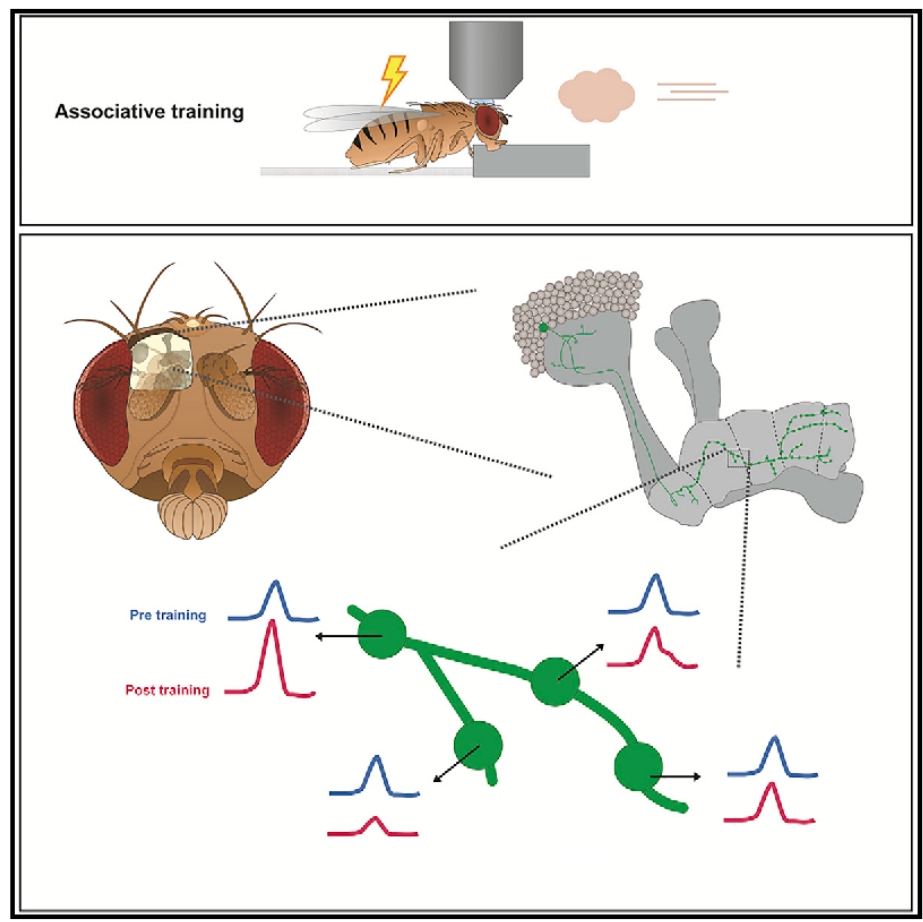

Highlights

- Calcium activity is monitored in synaptic boutons of single Kenyon cells

- Kenyon cell boutons are individually modifiable and act as functional units

- Associative learning causes decorrelation of activity across synaptic boutons

- Learning induces a gain in information rate coding across synaptic boutons

\section{Authors}

Florian Bilz, Bart R.H. Geurten, Clare E. Hancock, Annekathrin Widmann, André Fiala

\section{Correspondence} afiala@gwdg.de

\section{In Brief}

Bilz et al. have used fruit flies to analyze how associative memories are encoded across distributed synapses within a brain. Using calcium imaging, they find that learning increases information throughput by changing coherent activity across synaptic boutons. 


\section{Article \\ Visualization of a Distributed Synaptic
Memory Code in the Drosophila Brain}

Florian Bilz, ${ }^{1,3}$ Bart R.H. Geurten, ${ }^{2,3}$ Clare E. Hancock, ${ }^{1,3}$ Annekathrin Widmann, ${ }^{1,3}$ and André Fiala ${ }^{1,4, *}$

${ }^{1}$ Department of Molecular Neurobiology of Behavior, Johann-Friedrich-Blumenbach-Institute for Zoology and Anthropology, University of Göttingen, Julia-Lermontowa-Weg 3, 37077 Göttingen, Germany

2Department of Cellular Neurobiology, Johann-Friedrich-Blumenbach-Institute for Zoology and Anthropology, University of Göttingen, Julia-Lermontowa-Weg 3, 37077 Göttingen, Germany

${ }^{3}$ These authors contributed equally

${ }^{4}$ Lead Contact

*Correspondence: afiala@gwdg.de

https://doi.org/10.1016/j.neuron.2020.03.010

\section{SUMMARY}

During associative conditioning, animals learn which sensory cues are predictive for positive or negative conditions. Because sensory cues are encoded by distributed neurons, one has to monitor plasticity across many synapses to capture how learned information is encoded. We analyzed synaptic boutons of Kenyon cells of the Drosophila mushroom body $\gamma$ lobe, a brain structure that mediates olfactory learning. A fluorescent $\mathrm{Ca}^{2+}$ sensor was expressed in single Kenyon cells so that axonal boutons could be assigned to distinct cells and $\mathrm{Ca}^{2+}$ could be measured across many animals. Learning induced directed synaptic plasticity in specific compartments along the axons. Moreover, we show that odor-evoked $\mathrm{Ca}^{2+}$ dynamics across boutons decorrelate as a result of associative learning. Information theory indicates that learning renders the stimulus representation more distinct compared with naive stimuli. These data reveal that synaptic boutons rather than cells act as individually modifiable units, and coherence among them is a memory-encoding parameter.

\section{INTRODUCTION}

Deciphering how brain circuits, the neurons they consist of, and their synaptic connections acquire and encode learned information is a key task in modern neuroscience (Poo et al., 2016). Decades of research have led to our current understanding of changes in synaptic transmission as a key neuronal substrate underlying learning and memory formation (Hebb, 1949; Martin et al., 2000; Takeuchi et al., 2013; Josselyn and Frankland, 2018). Sensory stimuli that can be learned and memorized are encoded in the brain as neuronal activity that is sparsely distributed across ensembles of neurons and levels of processing. Physical changes in synaptic transmission underlying the encoding of a specific memory are, therefore, also distributed across many neurons and synapses (Josselyn and Frankland, 2018). It is assumed that, during learning, synaptic connections between neuronal ensembles that are active during the perception of a stimulus become modified such that their combined activity pattern can be retrieved during memory recall, thereby instructing future behavioral action (Josselyn and Frankland, 2018). The sparsely distributed nature of these memory traces (or engrams) makes it challenging to experimentally determine the rules by which many individual synaptic connections change. It is difficult to monitor plasticity in individual synapses at sufficient resolution and to observe many synapses comprehensively at the same time.

Here, we address this problem using the fruit fly Drosophila melanogaster, a key model organism for the analysis of neuronal substrates underlying learning and memory. The system is advantageous because it combines few but often genetically tractable neurons with a behavioral repertoire and neuronal complexity rich enough to allow for conceptual comparison with mammals. In Drosophila, classical olfactory conditioning (Tempel et al., 1983; Tully and Quinn, 1985) is a widely used learning paradigm (for review, see Davis, 1993; Heisenberg, 2003; Fiala, 2007; Busto et al., 2010, and Cognigni et al., 2018). In this training procedure, animals learn to avoid or approach a specific odor as a conditioned stimulus (CS) when it is temporally paired with a punishing or rewarding unconditional stimulus (US), such as an electric shock or sugar. Odors are detected by $\sim 1,320$ olfactory sensory neurons per hemisphere, located on the third antennal segments and maxillary palps, that project to the glomeruli of the antennal lobes (Vosshall and Stocker, 2007), which are the structural and functional analogs of the vertebrate olfactory bulbs (Hildebrand and Shepherd, 1997). Second-order olfactory projection neurons relay the processed odor information to the lateral horn and the mushroom body (MB) calyx (Tanaka et al., 2004); this structure forms the main sensory input region of the MB (Figure $1 \mathrm{~A}$ ), which consists of approximately 2,000-2,500 intrinsic neurons (Kenyon cells [KCs]; Technau, 1984; Aso et al., 2009). At the projection neuron-to-KC synapses, odor information is transformed from highly combinatorial neuronal activity (dense code)-analogous to the situation in the mammalian olfactory bulb-to a nonstereotypic (Murthy et al., 2008; Caron et al., 2013) and sparsely distributed pattern of KC activity (sparse code; Perez-Orive et al., 2002; Turner et al., 2008; Luo et al., 2010; Honegger et al., 2011; 

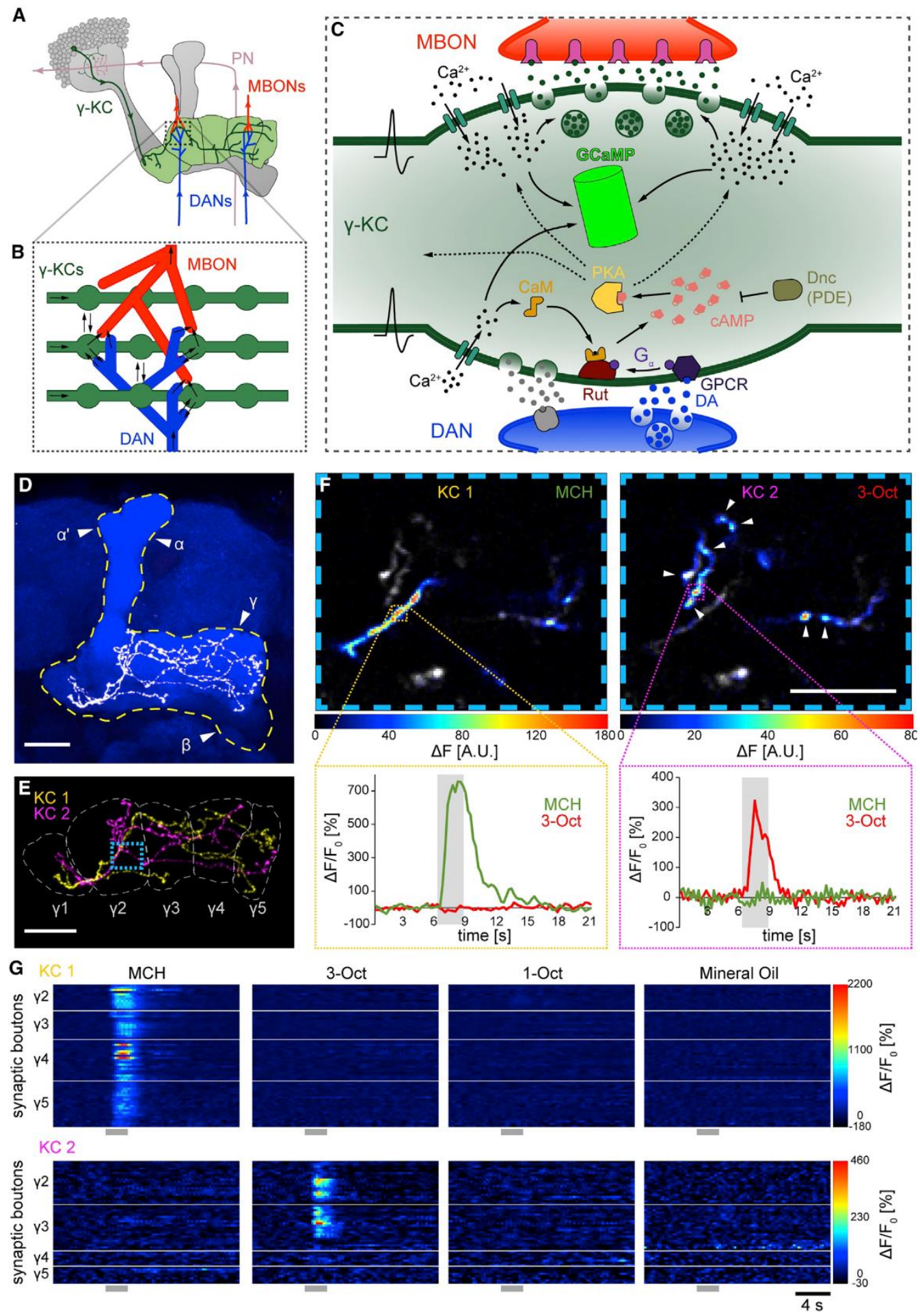

(legend on next page) 
Gruntman and Turner, 2013) - similar to the situation in the anterior piriform cortex of mammals (Sosulski et al., 2011). The parallel bundles of $\mathrm{KC}$ axons collectively form the MB lobes (Crittenden et al., 1998; Lee et al., 1999). The site of coincidence between the CS and US and the synaptic circuitry that underlies associative olfactory learning and short-term memory is confined to the $\gamma$ lobes (Zars et al., 2000; Blum et al., 2009; Qin et al., 2012), which contain approximately $650 \mathrm{KCs}$ per hemisphere (Aso et al., 2009). The $\gamma$ lobes are divided into five zonal compartments $(\gamma 1-\gamma 5)$, each of which is defined by the dendritic innervations of only one or two MB output neurons (MBONs), whose dendritic trees integrate information across multiple $\mathrm{KCs}$ (Aso et al., 2014a). MBONs show behavior-instructive properties: optogenetic activation of MBONs that innervate the $\gamma 1$, $\gamma 2$, or $\gamma 3$ compartments induces behavioral attraction toward the light source, whereas optogenetic activation of MBONs that innervate the $\gamma 4$ and $\gamma 5$ compartments induces behavioral repulsion from light (Aso et al., 2014b). In addition, the $\gamma$ lobes also receive compartmentalized input from dopaminergic neurons (DANs) (Figures $1 \mathrm{~A}$ and $1 \mathrm{~B}$ ), which exert punishing or rewarding US-signaling properties (Burke et al., 2012; Liu et al., 2012; Riemensperger et al., 2005; Schwaerzel et al., 2003), similar to the function of DANs in mammals (Schultz et al., 1997; Wise, 2004). Dopaminergic neurons that innervate the $\gamma 1$ and $\gamma 2$ compartments (Pech et al., 2013) mediate punishment (Schwaerzel et al., 2003; Riemensperger et al., 2005; ClaridgeChang et al., 2009; Mao and Davis, 2009; Dawydow et al., 2014; Cohn et al., 2015). In contrast, DANs that innervate the $\gamma 4$ and $\gamma 5$ compartments signal reward (Burke et al., 2012; Lit et al., 2012; Cohn et al., 2015). Importantly, synaptic plasticity induced by optogenetic stimulation of DANs is presynaptically localized to $\mathrm{KCs}$, but not postsynaptically localized to MBONs (Hige et al., 2015a). Postsynaptic changes in odor-evoked MBON activity as a result of associative learning or optogenetic activation of DANs have been determined electrophysiologically (Hige et al., 2015a) and by using optical $\mathrm{Ca}^{2+}$ imaging (Bouzaiane et al., 2015; Owald et al., 2015; Perisse et al., 2016; Berry et al., 2018; Yamazaki et al., 2018). However, learning-induced presynaptic plasticity in axonal KC synapses has never been directly observed. The sparsely distributed and stochastic nature of odor representations and the density of axonal $\mathrm{KC}$ fibers make an analysis of plasticity at the presynaptic level extremely chal- lenging. Thus, the rules by which $\mathrm{KC}$ presynapses change in order to convert a high-dimensional sensory code into a lowdimensional, behavior-instructing output remain unclear. To elucidate the changes that $\mathrm{KC}$ synapses undergo following associative learning, we used $\mathrm{Ca}^{2+}$ imaging to visualize synaptic activity in Drosophila at the exact integration point between the CS and US signals (i.e., at the axonal KC boutons; Figures $1 \mathrm{~A}-1 \mathrm{C})$.

\section{RESULTS}

Single $\mathrm{KC} \mathrm{Ca}^{2+}$ Imaging at the Synaptic Bouton Leve We implemented mosaic analysis with a repressible cell marker (MARCM) (Lee and Luo, 1999) to express the $\mathrm{Ca}^{2+}$ sensor GCaMP3 (Tian et al., 2009) in single $\gamma$ lobe KCs and mCherry (Shaner et al., 2004) as an anatomical marker. Transgene expression was limited to one to three $\mathrm{KCs}$ per brain hemisphere so that entire axons of individual KCs could be differentiated; this was achieved with a success rate of $50 \%$ ( $n=240$ flies in total). The flies had windows cut in their head capsules, were positioned under a two-photon microscope, and were stimulated with three different odorants (4-methylcyclohexanol, 3-octanol, and 1-octanol) or the odorant diluent (mineral oil). Several optical planes were monitored for each fly to ensure that as much of the entire axon was visualized as possible; as reported in other studies (e.g., Cohn et al., 2015), it was not possible to visualize the $\gamma 1$ compartment because of its optical inaccessibility. Brains were removed after $\mathrm{Ca}^{2+}$ imaging and subjected to immunohistochemical staining using an anti-Discs-large (DLG) antiserum and subsequent confocal microscopy, allowing for $3 \mathrm{D}$ reconstruction of KCs (Figures 1D and 1E; Video S1). This procedure enabled us to assign synaptic boutons along a particular KC axon to a specific compartment $(\gamma 2-\gamma 5$; Figures 1D-1G, S1, and S2; Video S2). We confirmed that the highly visible axonal swellings (Figure S2) represented synaptic boutons by ectopically expressing Bruchpilot, an active zone protein, tagged with GFP (Wagh et al., 2006) that predominantly localized to these boutons (Figure 2A). This is in accordance with the findings of electron microscopy (EM)-based connectome studies of a lobe $\mathrm{KCs}$ that have also described presynaptic machineries located in these boutons (Takemura et al., 2017). Of course, we do not exclude that synaptic output also occurs at smooth

Figure 1. $\mathrm{Ca}^{2+}$ Imaging of Synaptic Bouton Activity in Single KCs

(A) Schematic of mushroom body (MB) $\gamma$ lobe connectivity. $\gamma-\mathrm{KC}, \gamma$ lobe Kenyon cell; DANs, dopaminergic neurons; MBONs, MB output neurons; PN, projection neuron.

(B) Schematic magnification of synaptic connectivity. Arrows indicate direction of synaptic transmission.

(C) Intracellular cascades within synaptic boutons. CaM, calmodulin; DA, dopamine; Dnc, phosphodiesterase (PDE) encoded by the dunce gene; GPCR, G-protein-coupled receptor; PKA, protein kinase A; Rut, adenylate cyclase encoded by the rutabaga gene. GCaMP is used to monitor Ca ${ }^{2+}$. Dashed arrows indicate indirect influences.

(D) Axons of two $\gamma$ lobe KCs. Anti-DLG immunoreactivity is shown in blue. Dashed yellow line indicates the MB. The arrowheads indicate $\alpha, \alpha^{\prime}, \beta$, and $\gamma$ lobe. (E) $3 \mathrm{D}$ reconstruction of the two KCs shown in (D) and indication of the $\gamma$ lobe compartments $\gamma 1-\gamma 5$. The blue rectangle indicates an approximate region of interest for which $\mathrm{Ca}^{2+}$ dynamics are illustrated in $(\mathrm{F})$.

(F) Top: $\mathrm{Ca}^{2+}$ transients (false-color-coded change in GCaMP fluorescence) in parts of the $\mathrm{KC}$ axons in $\gamma 2$ evoked by the odorants 4-methylcyclohexanol (MCH) and 3-octanol (3-Oct). Bottom: dynamics of $\mathrm{Ca}^{2+}$ transients (odor duration indicated by shaded gray areas) are shown. White arrowheads exemplify synaptic boutons. See also Videos S3 and S4.

(G) $\mathrm{Ca}^{2+}$ dynamics in all synaptic boutons along the two $\mathrm{KC}$ axons. Each line represents one synaptic bouton that was assigned to an axonal compartment, $\gamma 2-\gamma 5$. Odor stimulation is indicated by the gray bars.

Scale bars: $20 \mu \mathrm{m}$ 

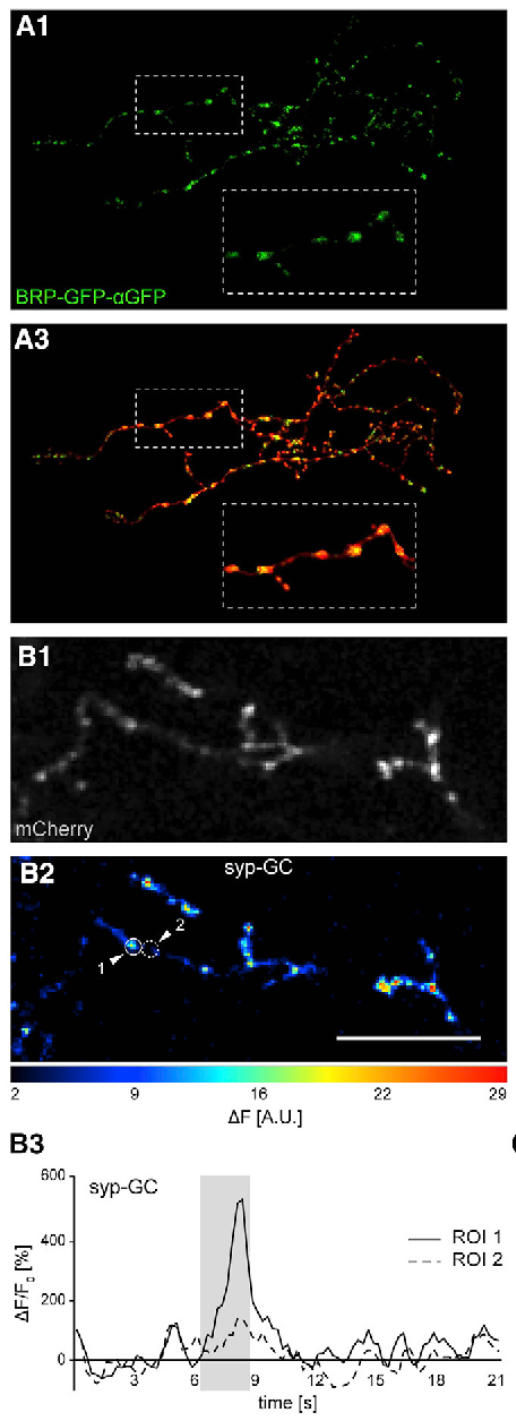

\section{C3}
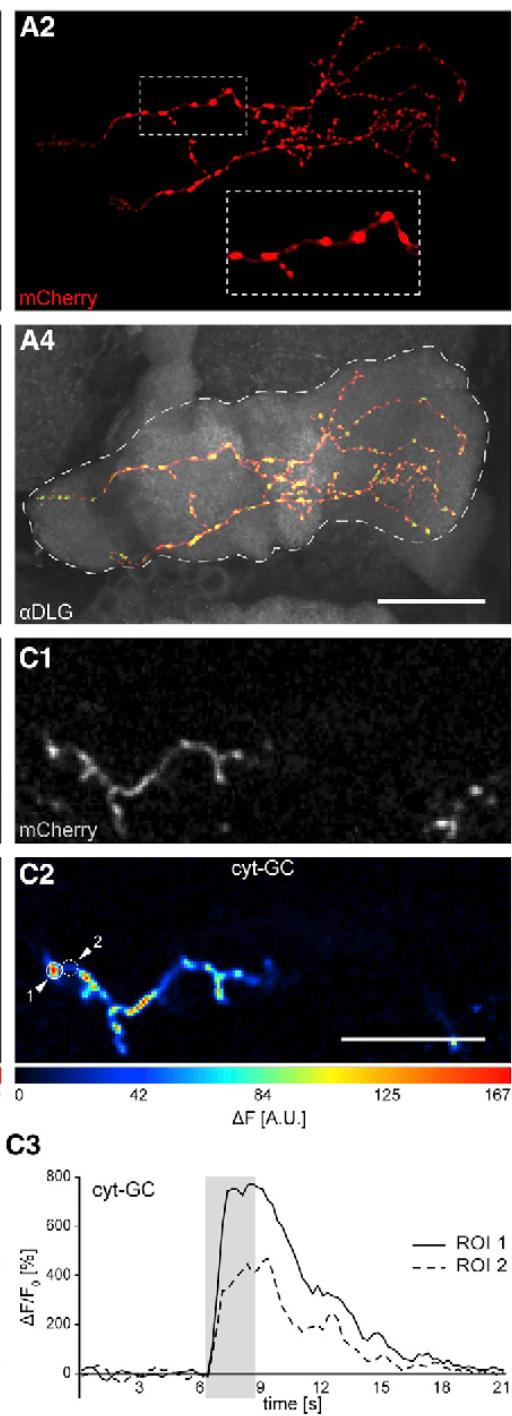

Figure 2. The Presynaptic Active Zone Protein BRP Localizes to Single KC Boutons (A1-A4) The maximum projection across a z stack of confocal microscopic images shows that Bruchpilot (BRP) localizes to boutons in $\gamma$ lobe KCs. (A1) BRP-GFP expressed in two single $\gamma-\mathrm{KCs}$ immunostained against GFP (green) shows labeling in boutons. (A2) mCherry expression (red) of the same two $\gamma$-KCs shows the axonal branches with its boutons. (A3) Merge of $A 1$ and $A 2$ shows that BRP-GFP is mainly present in the boutons (yellow). Insets of A1-A3 (dashed rectangles) show a 1.8X magnification of an axonal branch. (A4) Overlay of $\mathrm{A} 3$ and the staining against the postsynaptic protein DLG (gray) indicate the MB and the $\gamma$ lobe (dashed outline).

(B1-B3) $\mathrm{Ca}^{2+}$ imaging of a single focal plane. (B1) mCherry fluorescence is shown. (B2) False-colorcoded change in Syp-GCaMP fluorescence is shown. (B3) $\mathrm{Ca}^{2+}$ transients are shown for two regions of interest (ROI1 or $\mathrm{RO} / 2$ ) indicated in $\mathrm{B} 2$ covering an axonal bouton and an axon fraction next to a bouton.

(C1-C3) $\mathrm{Ca}^{2+}$ imaging of a single focal plane. (C1) mCherry fluorescence is shown. (C2) False-colorcoded change in cytosolic GCaMP fluorescence is shown. (C3) $\mathrm{Ca}^{2+}$ transients are shown for two regions of interest (ROl1 or $\mathrm{RO} 2$ ) indicated in $\mathrm{C} 2$ covering an axonal bouton and an axon fraction next to a bouton.

Scale bars: $20 \mu \mathrm{m}$.

responsive and non-responsive $\mathrm{KCs}$ that could be analyzed in detail illustrated the sparsely distributed nature of KC activity (Figure S3).

Axonal KC $\gamma$ Lobe Compartments $\gamma 2-\gamma 4$ Encode Odors as Functional Units

Of the 49 responsive $\mathrm{KCs}$, the axons of 31 $\mathrm{KCs}$ containing 38-93 synaptic boutons across $\gamma 2-\gamma 5$ compartments (mean \pm $\mathrm{SD} ; 62.74 \pm 12.61$ ) could be anatomically and functionally characterized in detail

axonal parts between boutons. Odor-evoked $\mathrm{Ca}^{2+}$ activity using presynaptically targeted Syp-GCaMP (Pech et al., 2015) was preferentially localized to these boutons (Figures $2 \mathrm{~B}$ and $2 \mathrm{C}$ ). The cytosolic GCaMP3 (Tian et al., 2009) also showed odorevoked $\mathrm{Ca}^{2+}$ influx between boutons but with lower amplitudes than within boutons (Figures $1 \mathrm{~F}$ and $2 \mathrm{C}$ ). Out of $270 \mathrm{KCs}$ from 120 flies, 49 showed robust responses to one or more of the three tested odorants or the diluent (see Videos S3 and S4 for examples). These data were in accordance with previously reported degrees of sparseness (Honegger et al., 2011) but showed odorant-specific differences at the concentrations used. Specifically, $8.1 \%$ of $\gamma$ lobe KCs responded to 4-methylcyclohexanol, $10.0 \%$ to 3 -octanol, $4.1 \%$ to 1 -octanol, and $2.2 \%$ to the diluent mineral oil. Averaged across all synaptic boutons, the temporal dynamics of odor-evoked $\mathrm{Ca}^{2+}$ transients of 139
(Figures 3 and S1) such that synaptic boutons could be unambiguously assigned to specific MB compartments. This revealed that odor-evoked $\mathrm{Ca}^{2+}$ influx did not always occur homogeneously at all boutons and compartments along a given $\mathrm{KC}$ axon. Rather, odor-evoked activity was frequently restricted to defined MB compartments (Figures $1 \mathrm{~F}, 1 \mathrm{G}$, and 3). KCs often showed pronounced odor-evoked $\mathrm{Ca}^{2+}$ transients in selective compartments (e.g., KC 17 and $\mathrm{KC} \mathrm{7;} \mathrm{illustrated} \mathrm{in} \mathrm{Figure} \mathrm{3A).}$ However, in some instances, responses were more homogeneous across the entire axon (e.g., KC 25; illustrated in Figure 3A). Odor representations in the MB are often described as active populations of $\mathrm{KCs}$, as measured in terms of somatic $\mathrm{Ca}^{2+}$ activity (e.g., Campbell et al., 2013; Lin et al., 2014). Our data showed that activation of $\mathrm{KCs}$ is not conveyed in a straightforward manner to all output regions and an additional 


\section{Article}
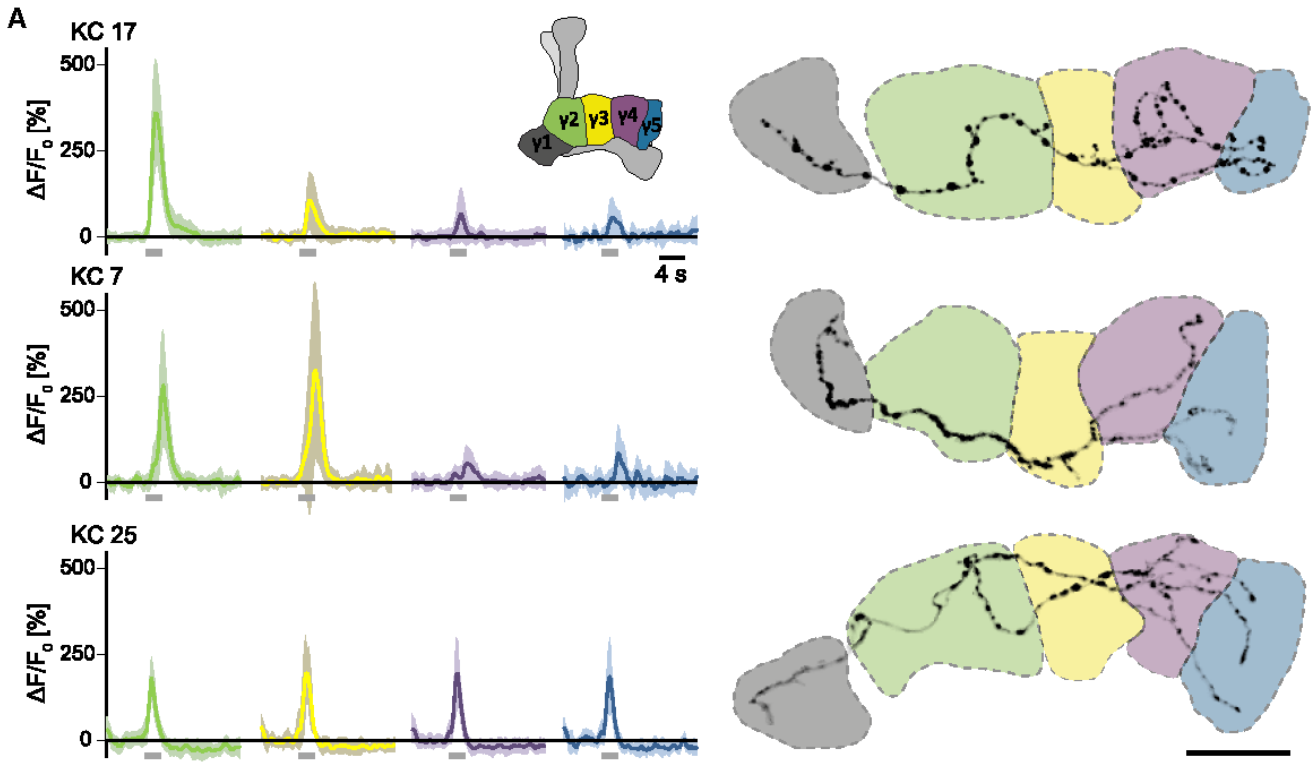

B

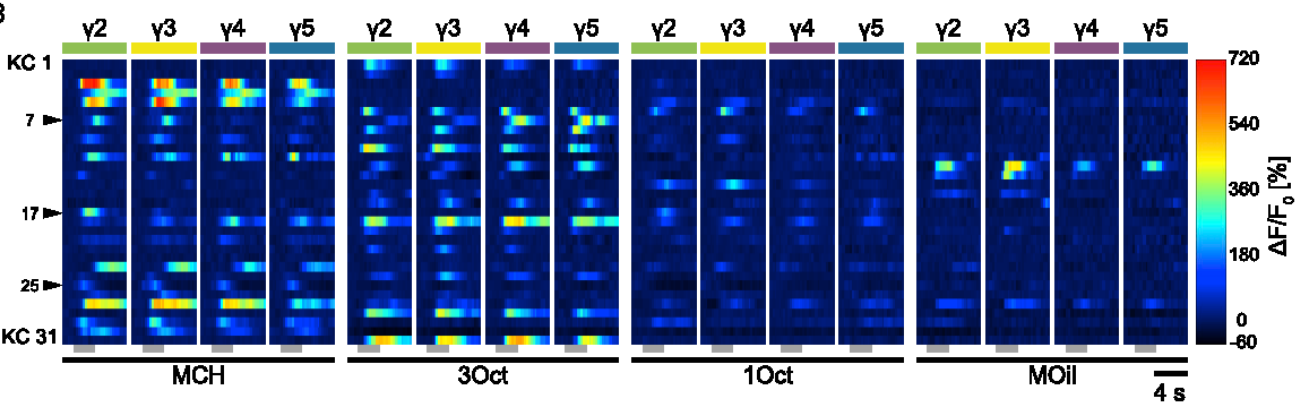

Figure 3. Single $\gamma$-KCs Show Compartment-Specific Odor Responses

(A) Exemplary traces of three KCs that showed odor-evoked $\mathrm{Ca}^{2+}$ dynamics in synaptic boutons of four axonal compartments, $\gamma 2-\gamma 5$. Lines indicate mean values, and shaded areas indicate the standard deviation $(n=10-39$ boutons per compartment). Odor stimulation is indicated by the gray bars along the time axis. On the right, the respective $\mathrm{KC}$ axons ( $\mathrm{z}$-projections) are shown. Scale bar: $20 \mu \mathrm{m}$.

(B) False-color-coded $\mathrm{Ca}^{2+}$ dynamics in axonal $\gamma$ lobe compartments in 31 different $\mathrm{KCs}$ responsive to any of the three tested odorants and the solvent mineral oil. Each row represents the median fluorescence intensity across all synaptic boutons within each compartment. Odor stimulation is indicated by the gray bars. The numbers and arrowheads on the left side indicate the three KCs that are presented in (A).

level of complexity is superimposed at the level of axonal boutons. Odors were represented as the combination of active boutons within distinct lobe compartments of individual KCs (Figure 3B). However, there was no statistically significant difference in the overall odor-evoked $\mathrm{Ca}^{2+}$ activity among $\gamma$ lobe compartments across all $31 \mathrm{KCs}$ (Figure 4A). The net $\mathrm{Ca}^{2+}$ activity remained equal among all measured $\gamma$ lobe compartments.

It is difficult to explain the observed heterogeneity in responsiveness ("odor tuning") between boutons and even within the same neurons, if one regards $\mathrm{KCs}$ as homogeneously acting, functional units that simply respond to odors. Extrinsic influences, such as dopamine release onto boutons, could affect the degree of odor-evoked $\mathrm{Ca}^{2+}$ influx. Because the axonal arborizations of DANs are confined to compartments (Aso et al., 2014a), as a first step, we investigated whether the formation of these boundaries along the $\gamma$ lobe axons has functional consequences for odor coding. We asked whether those boutons located within the same compartment respond naively to odors independent from each other or as coherent functional units defined by compartment. To quantify the variability between bouton responses or, conversely, the congruence as a measure of "similarity" between $\mathrm{Ca}^{2+}$ signals across synaptic boutons, we took into account both the amplitudes of odor-evoked $\mathrm{Ca}^{2+}$ transients and the temporal correlations between them (Figures 4A-4C). Considering these two parameters together is reasonable because both are relevant to spatial and temporal dendritic integration by downstream neurons (i.e., MBONs; Stuart and Spruston, 2015). We multiplied the cross-correlation coefficient, which was normalized to the autocorrelation, with the Michelson 
A

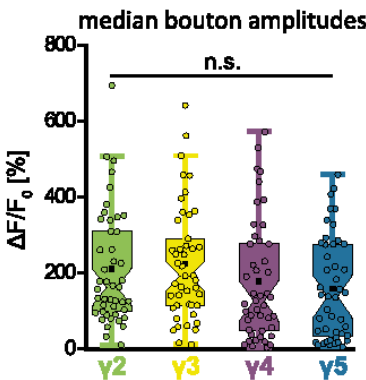

B

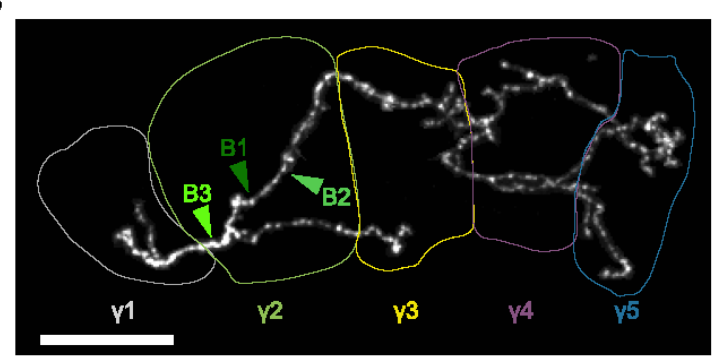

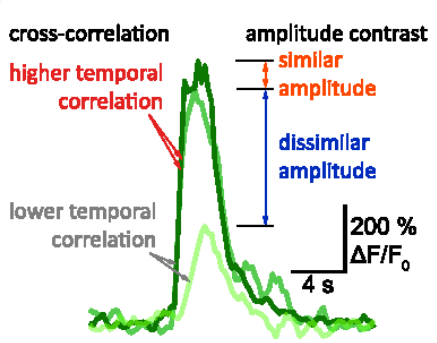

D

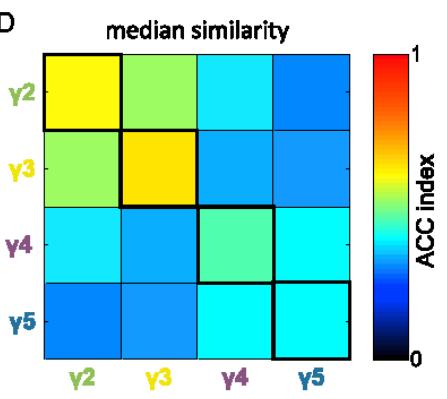

E median similarity contrast

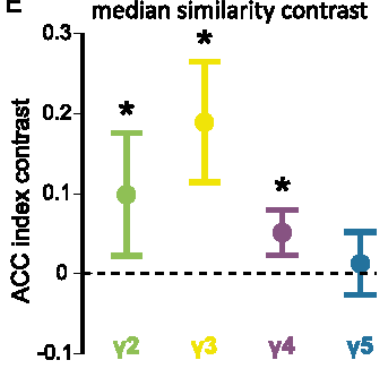

Figure 4. Axonal $\gamma$-KC Compartments Act as Functional Units for Encoding Odors

(A) Amplitudes of odor-evoked $\mathrm{Ca}^{2+}$ transients in $\gamma$ lobe compartments, $\gamma 2-\gamma 5$. Boxes indicate medians and $25 / 75$ percentiles, whiskers indicate $1.5 \times$ confidence intervals, and black dots indicate means. Colored dots indicate the median values for odor responses $(n=45)$ that were measured in $31 \mathrm{KCs}$. Some $\mathrm{KCs}$ responded to more than one odorant. No significant difference (n.s.) was detected between the compartments (Kruskal-Wallis test; $p>0.065$ ).

(B) $z$-projection of a single KC (KC1) (Figure 1D) and the corresponding compartments indicating the boutons (B1-B3) for which $\mathrm{Ca}^{2+}$ transients are shown in (C). Scale bar: $20 \mu \mathrm{m}$.

(C) Exemplary $\mathrm{Ca}^{2+}$ transients of the three boutons indicating a high temporal correlation and similar amplitudes between $\mathrm{B} 1$ and $\mathrm{B} 2$ and a lower temporal correlation and a strong difference in amplitude between B1 and B3.

(D) Congruence within and across the boutons of distinct compartments, indicated as the amplitude-corrected correlation (ACC) index. The ACC index of synaptic bouton activity within compartments exceeds the ACC index between compartments, except for $\gamma 5$.

(E) Quantification of the ACC index contrast as a measure for the difference between intra- and intercorrelation. The ACC contrast is 1 for a $\gamma$ lobe compartment whose boutons are only correlated with boutons of that compartment and -1 if each bouton is only correlated with boutons from other compartments. Median \pm $95 \%$ confidence intervals is indicated $(n=31)$. The intracompartment ACC index of synaptic bouton activity is significantly $\left(^{*} \mathrm{p}<0.05\right)$ higher than the intercompartment correlation in $\gamma 2-\gamma 4$, but not $\gamma 5$.

contrast of the odor-evoked $\mathrm{Ca}^{2+}$ response amplitudes and their corresponding means (see also STAR Methods section). This resulted in a numerical value ranging between 0 and 1 , which we termed the amplitude-corrected correlation (ACC) index. The ACC is 1 for identical responses and 0 if either amplitude of the two boutons is 0 (one cell not responding) or if the $\mathrm{Ca}^{2+}$ signals are not temporally correlated. Due to the amplitude-based terms, the ACC index rejects noise and offers a quantitative measure of congruence between $\mathrm{Ca}^{2+}$ patterns by combining the temporal domain of the response with its amplitude. We found that this ACC was higher within each axonal compartment $(\gamma 2-$ $\gamma 4$ ) than it was between compartments (Figures $4 \mathrm{D}$ and $4 \mathrm{E}$ ). That is, the synaptic boutons of each compartment acted together as a coherent unit. However, this was not the case for the $\gamma 5$ compartment, where the intracompartment bouton $\mathrm{Ca}^{2+}$ signals were no more correlated than the intercompartment signals. In conclusion, odor representations are transformed from sparsely activated $\mathrm{KC}$ assemblies into combinatorial activity of synaptic bouton assemblies that can act coherently as functional units $(\gamma 2-\gamma 4)$ or show only loosely congruent activity $(\gamma 5)$.
Associative Olfactory Learning Causes Decorrelation of Odor-Evoked Synaptic Bouton Activity within and across $\mathrm{KCs}$

To investigate learning-induced plasticity, we subjected 18 flies that expressed GCaMP in 20 responsive KCs to associative olfactory conditioning. Four $\mathrm{KCs}$ responded to both 4-methylcyclohexanol and 3-octanol that were used as conditioned stimuli, resulting in 24 different $\mathrm{KC}$ odor responses. After presentation of all three odorants and the diluent, one odor (CS+) was applied for $1 \mathrm{~min}$ and temporally paired with electric shocks (US) as punishment. A different odor (CS-) was then presented for 1 min but without the accompanying punishment. This experimental design is the most commonly used conditioning procedure in Drosophila (Tully and Quinn, 1985). We also used an additional control group, whereby flies were subjected to the same stimulation procedure without electric shocks (Figure 5A). Figure 5B shows learning-induced changes in $\mathrm{Ca}^{2+}$ activity for the two KCs illustrated in Figures 1D-1G. KC 1 responded to CS+, and the evoked $\mathrm{Ca}^{2+}$ influx decreased significantly in compartments $\gamma 3-\gamma 5$ after training. By contrast, $\mathrm{KC} 2$ responded to $\mathrm{CS}-$, and 
Neuron

Article

A training

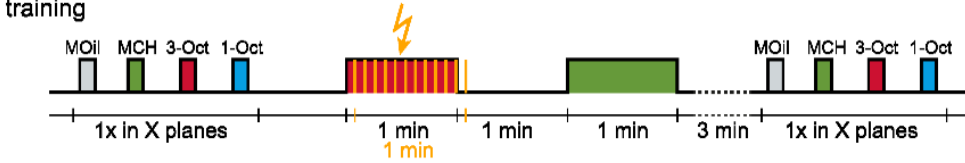

odor only

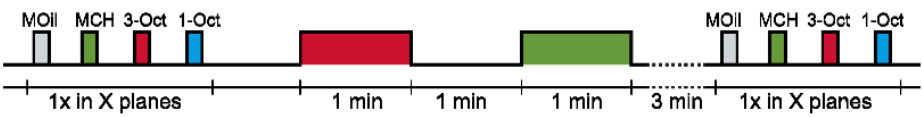

B
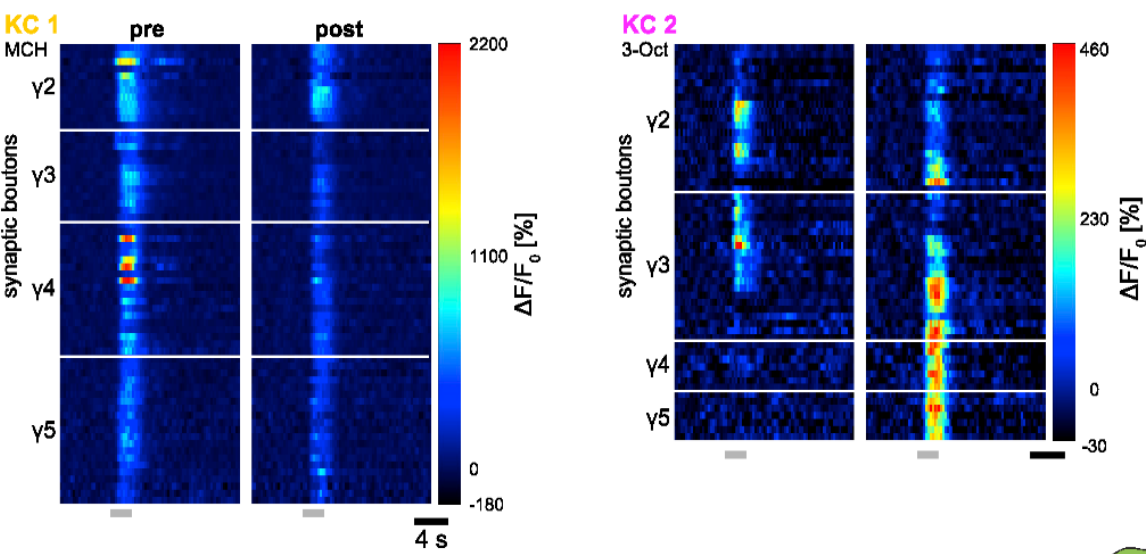

C
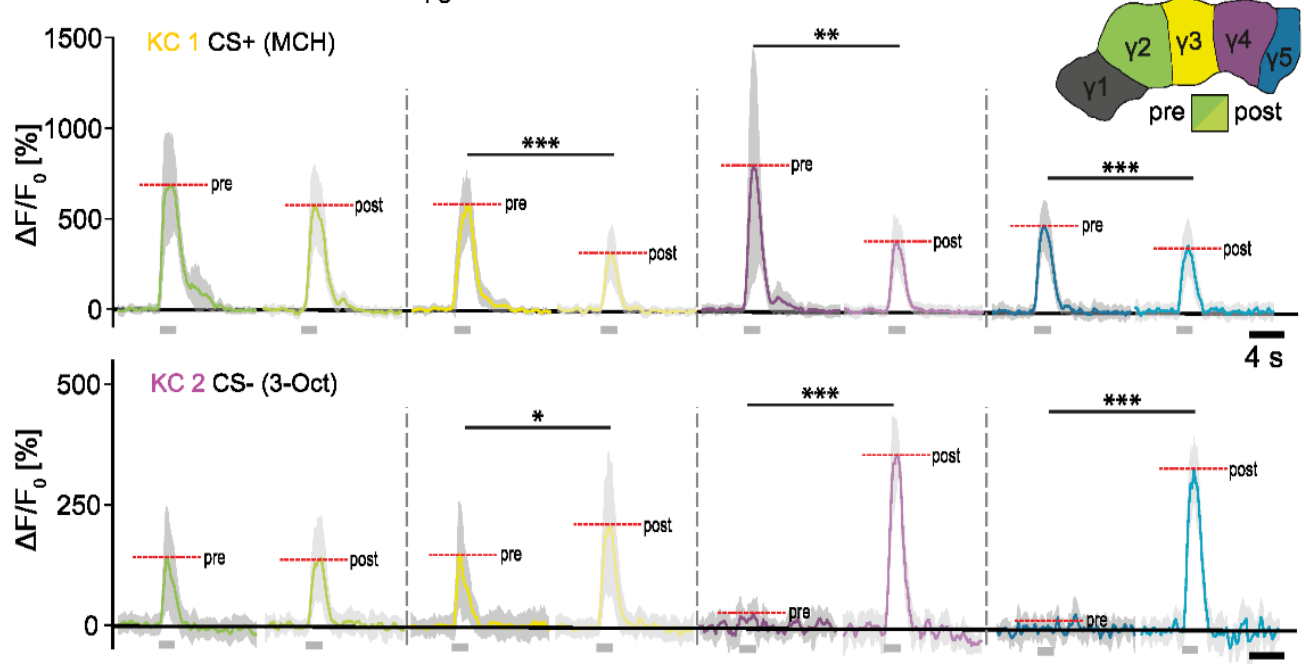

Figure 5. Associative Learning Modifies Compartment-Specific Odor Responses

(A) Schematic of the associative training regime. For clarity, only one permutation of odorant stimulation is shown.

(B) Odor-evoked $\mathrm{Ca}^{2+}$ transients in synaptic boutons within $\gamma$ lobe compartments $\gamma 2-\gamma 5$ for the two KCs of the same fly shown in Figures $1 \mathrm{D}-1 \mathrm{~F}$ before (pre) and after (post) training. $\mathrm{KC} 1$ responds to $\mathrm{MCH}$, here used as the $\mathrm{CS}+$ condition, and $\mathrm{KC} 2$ responds to 3-Oct, used as the CS- condition.

(C) Temporal dynamics of odor-evoked $\mathrm{Ca}^{2+}$ activity in all boutons shown for the odor representations pre- and posttraining. Traces indicate means across boutons $\pm S D(n=7-21)$. The pre- and posttraining amplitudes are highlighted as dashed red lines. Significances were tested with Fisher's permutation test, corrected by Benjamini-Hochberg false discovery rate (FDR) (KC1 p values: $\gamma-2: 0.0981, \gamma-3: 0.0002, \gamma-4: 0.0058, \gamma-5: 0.0008$; KC2 p values: $\gamma$-2: 0.1646, $\gamma-3$ : $0.0172, \gamma-4: 0.0001, \gamma-5: 0.0001)$ 

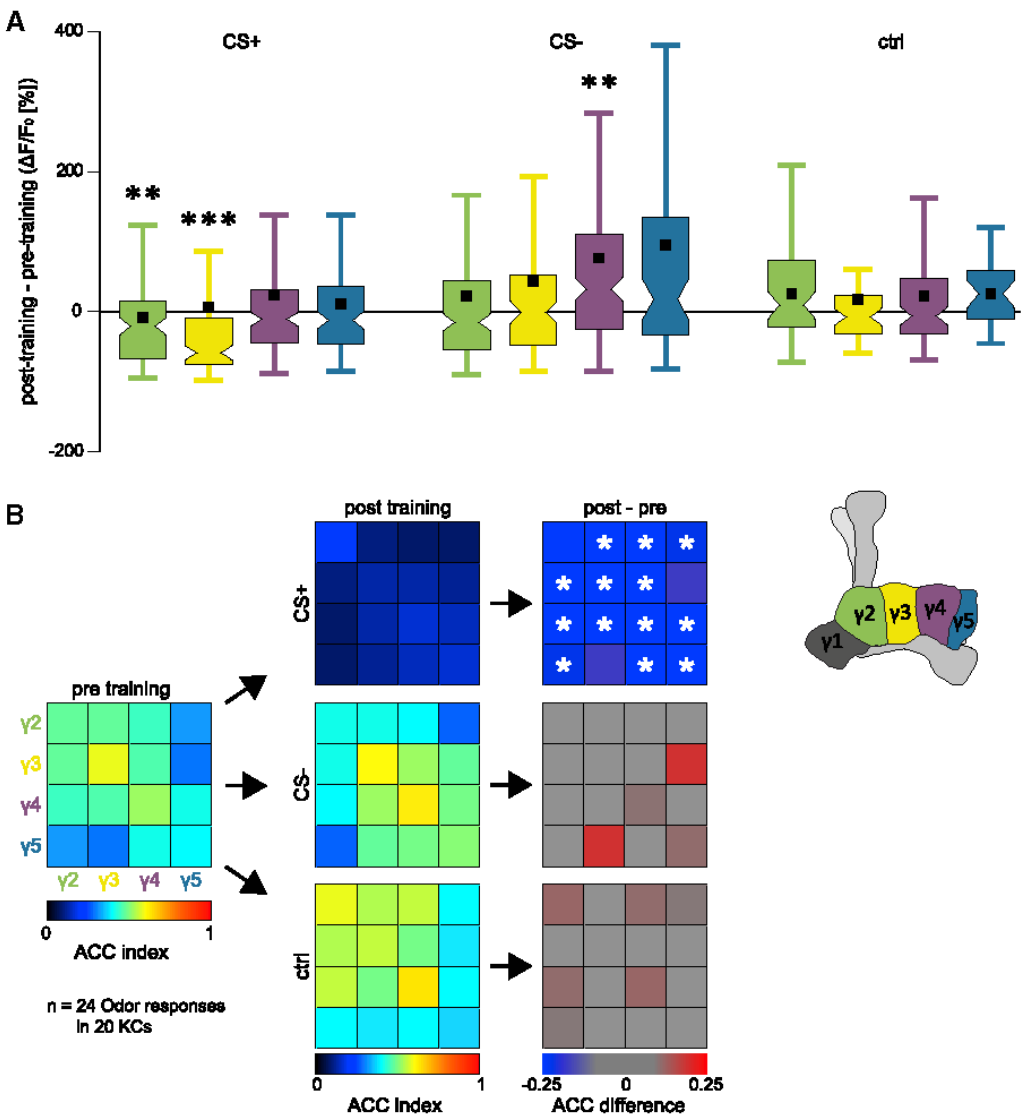

training caused a statistically significant recruitment of boutons located in compartments $\gamma 3-\gamma 5$ (Figures $5 B$ and $5 C$ ). This finding demonstrates that associative learning can induce antagonistic changes in $\mathrm{Ca}^{2+}$ influx in $\mathrm{KC}$ boutons within the same fly and that these changes can be restricted to specific axonal compartments. Comparison of the amplitudes of odor-evoked $\mathrm{Ca}^{2+}$ activity among all boutons measured before and after associative learning revealed bidirectional changes for both CS+ and CS-, but not homogeneous changes in only one direction (i.e., no uniform depression or facilitation; Figure S4). The amplitudes of odor-evoked $\mathrm{Ca}^{2+}$ transients did not differ significantly between pre- and posttraining conditions, most likely because of high variability (Figure S4). However, significantly more boutons in $\gamma 2$ and $\gamma 3$ exerted a decrease in odor-evoked $\mathrm{Ca}^{2+}$ activity than showed an increase after CS+ training (Figure $6 \mathrm{~A}$ ), potentially indicating decreased input to the cognate MBONs. By contrast, significantly more boutons increased their response after the CS- training in $\gamma 4$ (Figure $6 \mathrm{~A}$ ), potentially indicating increased drive to the cognate $\mathrm{MBON}$.

We utilized the ACC index as a measure of congruence to quantify how the overall representation of odor-evoked synaptic bouton activity within and across $\mathrm{KCs}$ changed over the course of associative training. We found large decreases in intra- and intercompartmental congruence for boutons responsive to CS+
Figure 6. Associative Learning Modifies the Congruence of Synaptic KC Boutons (A) Amplitude difference of odor-evoked $\mathrm{Ca}^{2+}$ signals across $\mathrm{KC}$ boutons for $\mathrm{CS}+, \mathrm{CS}$, or untrained flies (no US) as control (ctrl). Boxes indicate medians and $25 / 75$ percentiles, whiskers indicate $1.5 \times$ confidence intervals, and black dots indicate means. Significantly more boutons showed a decreased $\mathrm{Ca}^{2+}$ response after the training in $\gamma 2(n=100)$ and $\gamma 3(n=93)$ for the CS+ (sign test; ** $p<0.01 ;$;*t* $p<0.001$ ). This was not the case for $\gamma 4(n=155)$ and $\gamma 5(n=90)$. Significantly more boutons increased their response after the training in $\gamma 4(n=158)$ for the CS- (sign test, $\left.{ }^{* *} p<0.01\right)$. No statistically significant effects were found for $\gamma 2(n=139), \gamma 3(n=99)$, or $\gamma 5(n=75)$. No significant change was detected in the control (sign test; $p>0.58 ; \gamma 2: n=57 ; \gamma 3: n=47 ; \gamma 4$ $\mathrm{n}=90 ; \gamma 5: \mathrm{n}=29$ ).

(B) Color-coded amplitude-corrected correlation values ( $\mathrm{ACC}$ index) for $\mathrm{Ca}^{2+}$ dynamics of synaptic boutons within each compartment (internal correlation, diagonal) or across compartments. The congruence of $\mathrm{Ca}^{2+}$ activity within and across compartments decreased after training for CS+ but not for CS-, or the control (ctrl, no US) experiments. Asterisks indicate statistical significance. For median values and $95 \%$ confidence intervals, see Table $\mathrm{S} 1$.

which were statistically significant for most, but not all, intra- and intercompartment comparisons (Figure 6B). However, there was no corresponding decrease in congruence for boutons responsive to $\mathrm{CS}$ - or in the control condition. Because the effect of synaptic bouton activity occurred only in the CS+ condition and not in the CS- or odor-only control presentations, it is a result of associative conditioning. We conclude that the overall CS+ representation is differentiated from other odor representations via the correlation of the synaptic bouton activity. That this effect is due to combined changes in amplitude and temporal decorrelation (Figures 6A and S5) is suggested by the finding that it was not obvious in either the amplitude or temporal correlation alone; significant differences between CS+ and CSrepresentations became visible only after taking both parameters into account, suggesting that information is added by combining these two aspects. In conclusion, aversive associative olfactory conditioning induces not only a compartment-specific change in the amplitude of odor-evoked bouton activity but also a functional decorrelation of odor-evoked synaptic bouton activity both within and across KCs that extends across most, but not all, $\gamma$ lobe compartments. The representation of an odor that is learned as valence predictive at the level of distributed axonal bouton activity (i.e., the combinatorial synaptic odor code) is modified through associative learning such that coherent cooperation among boutons decreases. To test whether it is the temporal contiguity between CS and US and the learned valence that determines the decorrelation of bouton activity, we repeated the experiment but with the electric shock 
preceding the odor stimulation (backward conditioning), a training regime often used as a control (Tully and Quinn, 1985). However, backward conditioning is not neutral. In contrary to the "regular" conditioning regime with an overlapping CS-US coincidence, backward conditioning induces a learned attraction toward the trained odor, interpreted as "relief from punishment" learning (Tanimoto et al., 2004; König et al., 2018). This effect diminishes as the time between US and CS is increased (Yarali et al., 2012), which we tested as well. The regular "forward" conditioning was repeated again in parallel. Altogether, $12 \mathrm{KCs}$ out of $183 \mathrm{KCs}$ in 293 additionally tested flies were responsive and were monitored (Figure S6). Both forward and backward conditioning caused a significant decrease in the ACC index (Figure S6). A larger time delay between CS and US does not render a significant reduction of the ACC index, although residual effects can still be observed (Figure S6). We conclude that the decrease in congruence between and across synaptic bouton activities demarcates the learned odor as different from other, untrained odors, independent of the learned valence.

Odor-Evoked Synaptic Axonal Bouton Activity within and across KCs Forms Response Clusters

Our data show that synaptic boutons can exert coherent, correlated odor-evoked activity within distinct axonal compartments and a learning-induced loss of this coherence. This suggests a functional clustering of synaptic bouton activity. To test this hypothesis, we identified such clusters within our large array of bouton response traces. We used an agglomerative cluster analysis that operates on the odor-evoked bouton response amplitudes and their temporal latencies during pretraining. In this 2D data space (amplitude versus latency), the algorithm identified four distinct bouton response classes (BRCs) in our dataset (Figures 7A and 7B). BRC1 had the highest amplitude, BRC2 an intermediate amplitude, BRC3 a low amplitude and long latency, and BRC4 a low amplitude and a short latency (Figures 7A and 7B). Each BRC contained boutons from across the $\gamma$ lobe, with the highest proportion of boutons falling into BRC4 (Figure 7C). This distribution of BRCs changed after associative training; more boutons fell into the two BRCs with the highest $\Delta \mathrm{F} / \mathrm{F}_{0}$ amplitudes (BRC1 and $\mathrm{BRC}$ ) in the CS+ condition (Figures 7C and 7D), conversely leading to significant decreases in the other BRCs. This resulted in an overall broader distribution of bouton responses across BRCs for the CS+ condition (Figures 7C and 7D). The increase in BRC distribution is accompanied by an increase in variance within clusters, further corroborating the diversification of responses (Figure $7 \mathrm{E}$ ). However, in the CS- and odor-only conditions, the majority of synaptic bouton responses fell into cluster BRC4 and only a small fraction of responses fell into cluster BRC1, closely resembling the bouton distribution prior to associative conditioning (Figure $7 \mathrm{C}$ ). Therefore, "untrained" odor-evoked responses can be described by two distinct response classes; in contrast, "trained" odor responses require four much broader classes to be sufficiently described. This indicates a specific increase in the diversity of bouton responses to an odor that has been conditioned, compared to odors that are not explicitly assigned a value.
Utilization of the MB $\gamma$ Lobe Coding Space Is Expanded through Associative Learning

The outcome of classical conditioning is an altered behavioral response to a stimulus that differs from other stimuli in that it has gained predictive value. This implies that the learned stimulus evokes a neuronal representation that carries new information that was not present before conditioning. Having shown that olfactory responses are not homogeneously distributed across $\mathrm{KC}$ boutons and instead form distinct, categorical response classes (Figure 7A), it is possible to use Shannon's information theory (Shannon, 1949) to investigate exactly this concept. By considering each bouton as an independent variable, capable of falling into one of four classes (by membership in BRC1, BRC2, BRC3, or BRC4) and the distribution of boutons therein, it is possible to infer the bitwise information storage space of the $\gamma$ lobe and its utilization under different conditions. The distribution of responses can be quantified as entropy, which is a measure of variance for categorical data and describes how many bits (binary choices) are needed to encode information (Shannon, 1949). The application of information theory has been shown to be highly effective in judging the coding efficiency of neuronal systems (e.g., Grewe et al., 2007; Niven et al., 2007). In our case, the entropy quantifies how many bits characterize the $\gamma$ lobe $K C$ representation of an odor, based on our measured parameters only. Figure $7 \mathrm{~F}$ illustrates the median entropy calculated for each $\gamma$ lobe compartment. Before associative training, the four $\gamma$ lobe compartments showed comparably similar entropy (Figure $7 \mathrm{~F}$ ). The response to an untrained stimulus requires $\sim 1.6$ bits, which amounts to 1.96 categories (2 BRCs; Figures $7 \mathrm{~F}$ and $7 \mathrm{G}$ ). But this changed fundamentally in the course of associative training. After training, all compartments use $\sim 1.9$ bits, which is equivalent to 4 BRCs, with $\gamma 4$ and $\gamma 5$ showing the greatest increase. By contrast, with the exception of $\gamma 3$, the information entropy evoked by the CS - condition was lower and was also lower throughout all compartments in the odor-only condition. By using an abstract information rate measurement, we show that the information in the response of KCs to a learned odor is increased and might demarcate the odor as predictive. From these findings, we conclude that the decorrelation of bouton responses across the $\gamma$ lobe (Figure $6 \mathrm{~B}$ ) and the broader dispersion of responses across BRCs (Figures 7C and 7D) constitute a mechanism by which the learned stimulus utilizes an expanded information coding space of the $\gamma$ lobe to facilitate the storage of new, important information.

\section{DISCUSSION}

This paper quantitatively determined stimulus-evoked $\mathrm{Ca}^{2+}$ dynamics at axonal synaptic boutons within individual KCs and distributed across KCs of the MB. These data unexpectedly revealed that boutons along $\mathrm{KC}$ axons are not uniformly activated by odors. Rather, individual boutons, even of the same neurons, show individualized responsiveness. However, boutons located within the same compartments $\gamma 2-\gamma 4$ showed a more coherent odor tuning, implicating that these compartments act as functional units, the borders of which are likely determined by input neurons, such as DANs. In fact, 
A

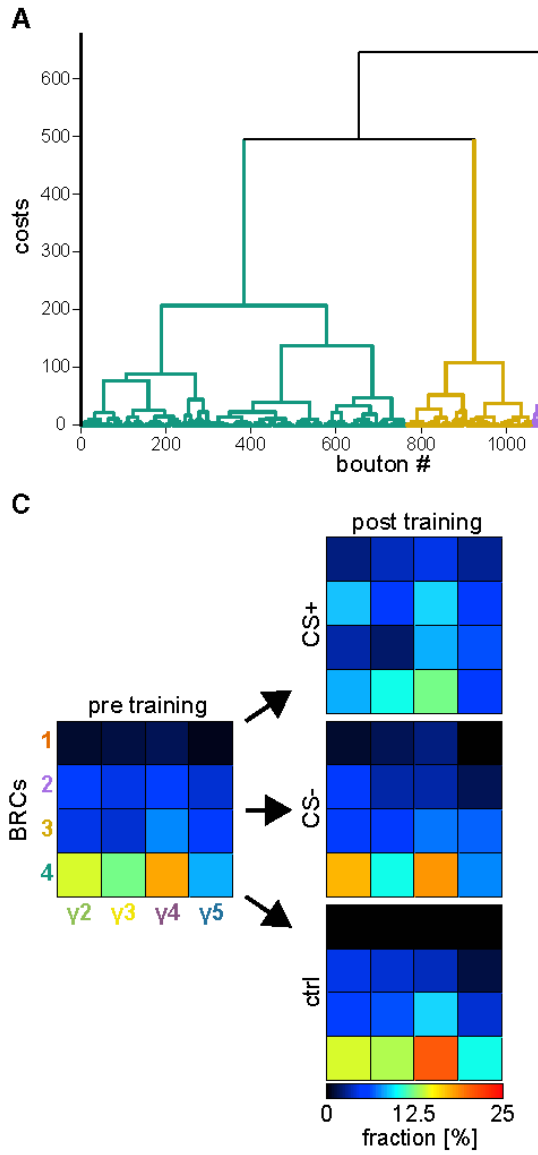

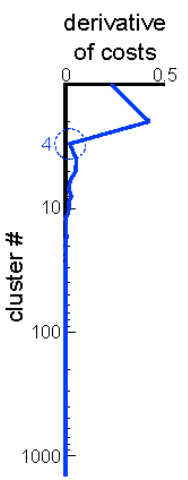

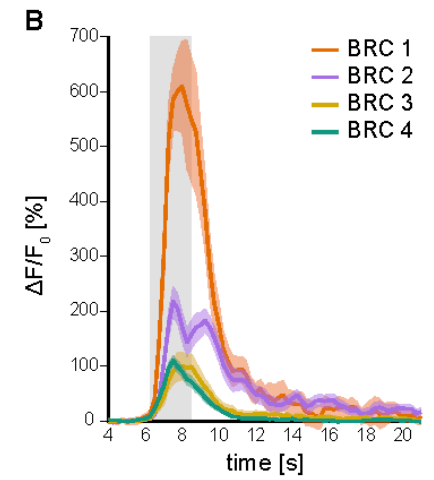

D
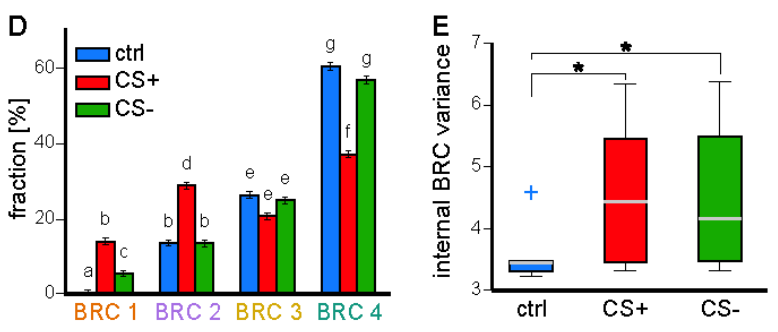

$\mathrm{F}$

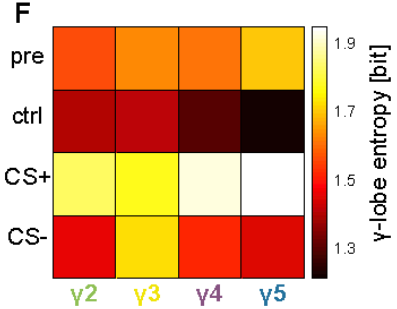

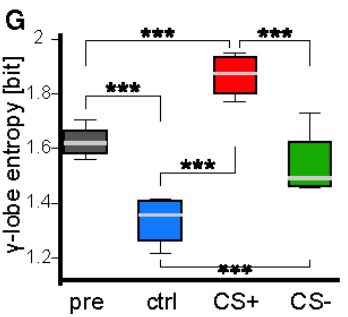

Figure 7. Categorization of Bouton Responses into Distinct Classes

(A) Agglomerative hierarchical clustering of the bouton $\mathrm{Ca}^{2+}$ responses (BRCs [bouton response classes]) before (pre) associative training. The sharp increase in the cost derivative (right) indicates the number of classes (dashed circle).

(B) Median pretraining $\mathrm{Ca}^{2+}$ responses of the corresponding BRCs of all boutons (median $\pm 95 \%$ confidence interval).

(C) Relative distribution of the four BRCs on the $\gamma$ lobe compartments, normalized to the sum of the BRCs ( $\mathrm{n}=24$ odor responses in $20 \mathrm{KCs} ; \mathrm{n}=1,457$ boutons). Associative training of an odor as CS+, CS-, or control (ctrl, no US; $n=8 \mathrm{KC}$ responses each) shifts the distribution of BRCs.

(D) Quantification of the fractions of the boutons in the BRCs on the four $\gamma$ lobe compartments after training. Bars indicate medians $\pm 95 \%$ confidence intervals; different letters indicate groups of different statistical significance $\left(p<0.01 ; X^{2}\right.$ test with Benjamini-Hochberg false discovery rate correction).

(E) The internal variance within each BRC is shown as the median value (gray vertical lines), interquartile range (colored boxes), and $1.5 \times$ interquartile distance (whiskers). Asterisks indicate statistical significance (Fisher's permutation test with Benjamini-Hochberg correction; ${ }^{\star} \mathrm{p}<0.05$ ).

(F) Quantification of the BRC dispersion with Shannon's information entropy, measured in bits, for each $\gamma$ lobe compartment before (pre) and after (post) training (G) Quantification of the training-induced change in information entropy across the entire $\gamma$ lobe for the CS+, CS-, or control (ctrl, no US) condition. Boxplots and statistical tests are as in $(E),{ }^{* *} \mathrm{p}<0.001$.

compartmentalized modulation of subcellular cyclic AMP (cAMP) levels in $\mathrm{KCs}$, driven by input DANs, has been shown (Boto et al., 2014). The $\gamma 5$ compartment represented an exception in that the $\mathrm{KC}$ boutons showed less correlated odorevoked activity, which might underlie the reported finding that $\gamma 5$ MBONs do not reliably respond to odors (Owald et al., 2015). The variability in odor responsiveness between individual boutons of even the same $\mathrm{KCs}$ is reminiscent of the plasticityand rutabaga-dependent individualization of MBONs (Hige et al., 2015b), which can be interpreted as a reflection of an animal's individual olfactory prior experience. Because MBONs are the direct downstream targets of axonal KC synapses, and dopamine-dependent plasticity has been shown to be presynaptically localized to KC boutons (Hige et al., 2015a), we suggest that the variability between boutons might represent the source of individualization of MBON responses. The concept that learning-dependent plasticity is localized to axonal KC presynapses and not to MBON postsynapses is also corroborated by reports that reversibly blocking transmitter release from KCs during training only does not prevent the acquisition of an associative memory (Dubnau et al., 2001; McGuire et al., 2001, 2003; Schwaerzel et al., 2002). 
Mechanistically, the phenomenon of functional individualization of synaptic boutons has been analyzed in motor neurons of the neuromuscular junctions in larval Drosophila (Maiellaro et al., 2016). Here, octopamine acts as a neuromodulator that alters cAMP levels in synaptic boutons, similar to the action of dopamine in KCs (Boto et al., 2014; Tomchik and Davis, 2009; Figure $1 \mathrm{C}$ ). The distribution of the phosphodiesterase dunce located at the peripheral rims of boutons restricts the diffusion of cAMP to individual boutons (Maiellaro et al., 2016). Because dunce is enriched in KCs (Nighom et al., 1991) and is required for proper olfactory learning (Davis, 1996), future research would be well placed to test whether dunce mediates functional individ ualization of synaptic boutons in KCs also. Individualized modulation of synaptic boutons, rather than entire neurons, is also reminiscent of the situation in mammals. Here, dendritic spines have been shown to be selectively modified in the course of learning, implying that synapses and spines represent basa elements of learning and memory formation rather than entire neurons (Poo et al., 2016).

To analyze how learning modifies the synaptically distributed odor representation, the animals were subjected to an aversive associative training procedure. Previous studies on MBONs have shown that pairing an odor with electric shock (Perisse et al., 2016; Berry et al., 2018) or artificial activation of punishment-mediating DANs (Hige et al., 2015a) leads to a depression of the activity of MBONs innervating the $\gamma_{1}$ and $\gamma^{2}$ compartments. The optogenetic activation of these MBONs confers behavioral attraction of animals toward the light source (Aso et al., 2014b). The function of the $\gamma 3$ compartment in associative learning is less clear (but see Yamagata et al., 2016). However, punitive electric shocks evoke activity in DANs innervating $\gamma 1$, $\gamma 2$, and $\gamma 3$, in contrast to a rewarding sugar stimulus that activates DANs targeting $\gamma 4$ and $\gamma 5$ (Cohn et al., 2015). Therefore, the predominant hypothesis in light of the above findings is that the relative activity of compartment-specific MBONs encodes the learned valence of odor stimuli (Cognigni et al. 2018; Hige, 2018; Owald and Waddell, 2015). In agreement with this hypothesis, we show that the median $\mathrm{Ca}^{2+}$ response amplitudes across the $\gamma 2$ and $\gamma 3$ compartments are reduced after aversive conditioning (Figure $6 \mathrm{~A}$ ), although we did not observe homogeneous depression but bidirectional modulation across these bouton populations. As a novel finding, we observed a complementary effect for the CS - condition; i.e., more boutons showed an increase in activity in the $\gamma 4$ compartment. It is well established that, in the course of differential olfactory conditioning, the CS- is learned as indicative for the absence of punishment (Barth et al., 2014; Schleyer et al., 2018). Our finding of opposite effects for CS+ and CS-, both in the direction of changes in $\mathrm{Ca}^{2+}$ activity and different $\gamma$ lobe compartments ( $\gamma 2$ versus $\gamma 4$ ), modifies the prevailing model of associative learning (see also Aso and Rubin, 2016). However, it must also be noted that our experiments differed from many previously reported approaches in two aspects. First, electric shock punishment was used as an US, in contrast to artificial activation of single DANs (Hige et al., 2015a; Aso and Rubin, 2016). Second, the animals were subjected to a training procedure while they were restrained under a microscope, allowing for a within-animal comparison of before and after training.
This approach differs from the comparison of untrained animals with animals $1 \mathrm{~h}$ after associative training (Owald et al., 2015; Perisse et al., 2016).

Our findings relate to one aspect of classical conditioning: the learned stimulus representation embodies behavior-instructive properties. Specifically, in the case of aversive olfactory conditioning, it induces repulsion from the odor source. However, a second aspect of associative learning is the "memory content." The specific stimulus representation (i.e., odor identity) must be demarcated as outcome predictive from other representations that do not carry any learned valence. The sparse nature of $\mathrm{KC}$ odor tuning and the relatively large array of $\mathrm{KCs}$ ensures a high degree of odor specificity (Honegger et al., 2011), although $\gamma$ type $\mathrm{KCs}$ are less selective compared with $\alpha / \beta$ and $\alpha^{\prime} / \beta^{\prime} \mathrm{KCs}$ (Inada et al., 2017). However, odor discriminability of individual $\mathrm{KCs}$ cannot be complete; $\mathrm{KCs}$ respond in some instances to more than one odorant, such as both the trained CS+ and $\mathrm{CS}-$ conditions. This was also the case in some synaptic $\mathrm{KC} \mathrm{re-}$ sponses to the odorants measured in this study (Figure S7). The degree of overlap between neuronal stimulus representations determines the degree to which a learned response toward one stimulus is generalized to a second stimulus (Niewalda et al., 2011; Barth et al., 2014). However, through differential training, flies can learn to disentangle similar odorants and increase their ability to differentiate between them (Barth et al. 2014). This phenomenon cannot be explained by gross facilitation or depression of $\mathrm{KC}$ presynapses. Here, we found that association of an odor with punishment caused a strong decorrelation of odor-evoked $\mathrm{Ca}^{2+}$ activity across boutons of most $\gamma$ lobe compartments, both between and within individual $\mathrm{KCs}$, and independent from valence (aversive punishment and appetitive relief learning). Therefore, overlapping $\mathrm{KC}$ boutons can be part of both the CS+ and CS - representations, dependent on correlations with the remaining synapses that encode the respective stimuli. We suggest that this could represent a mechanism by which synaptic interference between overlapping neuronal assemblies encoding for learned, aversive odors (CS+) and similar odors not predictive for punishment (CS-) may be circumvented. Our finding that synaptic boutons show decorrelated activity in response to a trained stimulus suggests that the particular odor representation is also uniquely demarcated from other odor representations. Coding theory suggests that stimulusspecific information is transmitted most efficiently if the units encoding the stimulus are decorrelated, reducing redundancy (Barlow, 1961). Exactly this is quantitatively shown here in the gain in entropy through associative training that we found for the synaptic odor representation. This principle is not confined to the MB or insect brains. It has, for example, recently been shown that, in the rat hippocampus, an increased variability in synaptic activity within populations of CA1 neurons as a result of learning increases the information coding ability of this brain region (Sakimoto et al., 2019).

We currently lack clear understanding of the physiological mechanisms that enable synaptic boutons to correlate or decorrelate $\mathrm{Ca}^{2+}$ activity both temporally and in amplitude, depending on whether the driving stimulus has been trained. Coherent neuronal activity, be it correlations between spike trains or between synaptic $\mathrm{Ca}^{2+}$ transients, is regarded as an emergent 
property of a neuronal circuit that typically depends on the balance between excitatory and inhibitory input (Litwin-Kumar et al., 2011; Doiron et al., 2016). Recent connectomic studies based on electron microscopic 3D reconstructions of MBs of larval and adult Drosophila have uncovered remarkable microcircuitry complexity at the axonal $\mathrm{KC}$ bouton level (Eichler et al., 2017; Takemura et al., 2017). For example, groups of KCs are synaptically interconnected with each other, and KC-MBON connections are often confined to Rosetta-like structures indicative of cooperative action of $\mathrm{KC}$ outputs (Takemura et al., 2017). The KC-KC and KC-MBON connections also show electrical coupling through gap junctions (Liu et al., 2016). Synchronous, correlated neuronal activity often depends on gap junctions, such as in the olfactory bulb of mammals (Pouille et al., 2017), in the antennal lobe of insects (Kazama and Wilson, 2009), or in the mammalian cortex (Pernelle et al., 2018). Thus, it is tempting to speculate that modulation of gap junctions might potentially affect correlative activity of $\mathrm{KC}$ boutons as a result of associative training. Regardless of the exact physiological mechanism, our data suggest that correlations at the synaptic circuit level contribute to encoding learned information, thereby combining the concept of a synaptic (rather than cellular) distribution of a memory code with that of correlated neuronal activity as a coding parameter.

\section{STAR $\star$ METHODS}

Detailed methods are provided in the online version of this paper and include the following:

- KEY RESOURCES TABLE

- RESOURCE AVAILABILITY

- Lead Contact

- Materials Availability

- EXPERIMENTAL MODEL AND SUBJECT DETAILS o Drosophila melanogaster

- METHOD DETAILS

O MARCM-mediated generation of single-cell clones

O Optical $\mathrm{Ca}^{2+}$ imaging

O Odor stimulation and olfactory conditioning

- Immunohistochemistry and confocal microscopy

o Agglomerative hierarchical clustering

- Amplitude-corrected correlation analysis

- QUANTIFICATION AND STATISTICAL ANALYSIS

- DATA AND CODE AVAILABILITY

SUPPLEMENTAL INFORMATION

Supplemental Information can be found online at https://doi.org/10.1016/]. neuron.2020.03.010.

\section{ACKNOWLEDGMENTS}

We are grateful to the Bloomington Drosophila Stock Center for providing fly strains. We would like to thank Yoshinori Aso, Bertram Gerber, and David Owald for helpful comments on the data and the manuscript. We thank the Department of Applied Mathematics (University of Goettingen) for providing MATLAB licenses. This work was supported by the German Research Council (FOR 2705 and SFB 889/B4), the Volkswagen Foundation, and the Ministry for Science and Culture of Lower Saxony (ZN 3014).

\section{AUTHOR CONTRIBUTIONS}

A.F. designed and supervised the study and wrote the manuscript with contributions from all authors; F.B., C.E.H., and A.W. performed experiments and analyzed the data; and B.R.H.G. designed and performed the computational analyses.

\section{DECLARATION OF INTERESTS}

The authors declare no competing interests.

Received: April 11, 2019

Revised: November 19, 2019

Accepted: March 13,2020

Published: April 7, 2020

\section{REFERENCES}

Allaj, E. (2018). Two simple measures of variability for categorical data. J. Appl. Stat. $45,1497-1516$

Aso, Y., and Rubin, G.M. (2016). Dopaminergic neurons write and update memories with cell-type-specific rules. eLife 5, e16135.

Aso, Y., Grübel, K., Busch, S., Friedrich, A.B., Siwanowicz, I., and Tanimoto, H. (2009). The mushroom body of adult Drosophila characterized by GAL4 drivers. J. Neurogenet. 23, 156-172.

Aso, Y., Hattori, D., Yu, Y., Johnston, R.M., lyer, N.A., Ngo, T.T., Dionne, H., Abbott, L.F., Axel, R., Tanimoto, H., and Rubin, G.M. (2014a). The neuronal ar chitecture of the mushroom body provides a logic for associative learning eLife 3, e04577.

Aso, Y., Sitaraman, D., Ichinose, T., Kaun, K.R., Vogt, K., Belliart-Guérin, G. Plaçais, P.Y., Robie, A.A., Yamagata, N., Schnaitmann, C., et al. (2014b). Mushroom body output neurons encode valence and guide memory-based action selection in Drosophila. eLife 3, e04580.

Barlow, H.B. (1961). Possible principles underlying the transformations of sensory messages. In Sensory Communication, W. Rosenblith, ed. (M.I.T. Press), pp. 217-233.

Barth, J., Dipt, S., Pech, U., Hermann, M., Riemensperger, T., and Fiala, A. (2014). Differential associative training enhances olfactory acuity in Drosophila melanogaster. J. Neurosci. 34, 1819-1837.

Benjamini, Y., and Hochberg, Y. (1995). Controlling the false discovery rate: a practical and powerful approach to multiple testing. J. R. Stat. Soc. B 57 , 289-300.

Berry, J.A., Phan, A., and Davis, R.L. (2018). Dopamine neurons mediate learning and forgetting through bidirectional modulation of a memory trace. Cell Rep. 25, 651-662.e5.

Blum, A.L., Li, W., Cressy, M., and Dubnau, J. (2009). Short- and long-term memory in Drosophila require CAMP signaling in distinct neuron types. Curr. Biol. 19, 1341-1350.

Boto, T., Louis, T., Jindachomthong, K., Jalink, K., and Tomchik, S.M. (2014). Dopaminergic modulation of CAMP drives nonlinear plasticity across the Drosophila mushroom body lobes. Curr. Biol. 24, 822-831.

Bouzaiane, E., Trannoy, S., Scheunemann, L., Plaçais, P.Y., and Preat, T. (2015). Two independent mushroom body output circuits retrieve the six discrete components of Drosophila aversive memory. Cell Rep. 11 1280-1292.

Burke, C.J., Huetteroth, W., Owald, D., Perisse, E., Krashes, M.J., Das, G. Gohl, D., Silies, M., Certel, S., and Waddell, S. (2012). Layered reward signalling through octopamine and dopamine in Drosophila. Nature 492, 433-437.

Busto, G.U., Cervantes-Sandoval, I., and Davis, R. L. (2010). Olfactory learning in Drosophita. Physiology (Bethesda) 25, 338-346.

Campbell, R.A., Honegger, K.S., Qin, H., Li, W., Demir, E., and Turner, G.C. (2013). Imaging a population code for odor identity in the Drosophila mushroom body. J. Neurosci. 33, 10568-10581. 
Caron, S.J., Ruta, V., Abbott, L.F., and Axel, R. (2013). Random convergence of olfactory inputs in the Drosophita mushroom body. Nature 497, 113-117.

Claridge-Chang, A., Roorda, R.D., Vrontou, E., Sjulson, L., Li, H., Hirsh, J., and Miesenböck, G. (2009). Writing memories with light-addressable reinforcement circuitry. Cell 139, 405-415.

Cognigni, P., Felsenberg, J., and Waddell, S. (2018). Do the right thing: neura network mechanisms of memory formation, expression and update in Drosophila. Curr. Opin. Neurobiol. 49, 51-58.

Cohn, R., Morantte, I., and Ruta, V. (2015). Coordinated and compartmentalized neuromodulation shapes sensory processing in Drosophita. Cell 163 $1742-1755$.

Crittenden, J.R., Skoulakis, E.M., Han, K.A., Kalderon, D., and Davis, R.L. (1998). Tripartite mushroom body architecture revealed by antigenic markers Learn. Mem. 5, 38-51.

Davis, R.L. (1993). Mushroom bodies and Drosophila learning. Neuron $11,1-14$.

Davis, R.L. (1996). Physiology and biochemistry of Drosophila learning mutants. Physiol. Rev. 76, 299-317.

Dawydow, A., Gueta, R., Ljaschenko, D., Ulrich, S., Hermann, M., Ehmann, N., Gao, S. Fiala, A., Langenhan, T., Nagel, G., and Kittel, R.J. (2014). Channelrhodopsin-2-XXL, a powerful optogenetic tool for low-light applications. Proc. Natl. Acad. Sci. USA 111, 13972-13977.

Doiron, B., Litwin-Kumar, A., Rosenbaum, R., Ocker, G.K., and Josić, K. (2016). The mechanics of state-dependent neural correlations. Nat Neurosci. 19, 383-393.

Dubnau, J., Grady, L., Kitamoto, T., and Tully, T. (2001). Disruption of neurotransmission in Drosophila mushroom body blocks retrieval but not acquisition of memory. Nature 411, 476-480.

Eichler, K., L., F., Litwin-Kumar, A., Park, Y, Andrade, I, Schneider-Mizell, C.M., Saumweber, T., Huser, A., Eschbach, C., Gerber, B., et al. (2017). The complete connectome of a learning and memory centre in an insect brain. Nature 548, 175-182.

Fiala, A. (2007). Olfaction and olfactory learning in Drosophila: recent progress Curr. Opin. Neurobiol. 17, 720-726.

Fouquet, W., Owald, D., Wichmann, C., Mertel, S., Depner, H., Dyba, M. Hallermann, S, Kittel, R.J., Fimer S, and Sigrist, S.J. (2009). Maturation of active zone assembly by Drosophila Bruchpilot. J. Cell Biol. 186 (1), 129-145.

Grewe, J., Weckström, M., Egelhaaf, M., and Warzecha, A.K. (2007). Information and discriminability as measures of reliability of sensory coding PLoS ONE 2, e1328.

Gruntman, E., and Turner, G.C. (2013). Integration of the olfactory code across dendritic claws of single mushroom body neurons. Nat. Neurosci. 16, 1821-1829.

Hebb, D.O. (1949). The Organization of Behavior: A Neuropsychological Theory (Wiley).

Heisenberg, M. (2003). Mushroom body memoir: from maps to models. Nat. Rev. Neurosci. 4, 266-275.

Hige, T. (2018). What can tiny mushrooms in fruit flies tell us about learning and memory? Neurosci. Res. 129, 8-16.

Hige, T., Aso, Y., Modi, M.N., Rubin, G.M., and Turner, G.C. (2015a) Heterosynaptic plasticity underlies aversive olfactory learning in Drosophila. Neuron 88, 985-998.

Hige, T., Aso, Y., Rubin, G.M., and Turner, G.C. (2015b). Plasticity-driven individualization of olfactory coding in mushroom body output neurons. Nature $526,258-262$.

Hildebrand, J.G., and Shepherd, G.M. (1997). Mechanisms of olfactory discrimination: converging evidence for common principles across phyla. Annu. Rev. Neurosci. 20, 595-631.

Honegger, K.S., Campbell, R.A., and Turner, G.C. (2011). Cellular-resolution population imaging reveals robust sparse coding in the Drosophila mushroom body. J. Neurosci. 31, 11772-11785.
Inada, K., Tsuchimoto, Y., and Kazama, H. (2017). Origins of cell-type-specific olfactory processing in the Drosophila mushroom body circuit. Neuron 95 , 357-367.e4.

Josselyn, S.A., and Frankland, P.W. (2018). Memory allocation: mechanism and function. Annu. Rev. Neurosci. 41, 389-413.

Kazama, $H_{\text {., }}$ and Wilson, R.L. (2009). Origins of correlated activity in an olfactory circuit. Nat. Neurosci. 12, 1136-1144.

König, C., Khalili, A., Ganesan, M., Nishu, A.P., Garza, A.P., Niewalda, T., Gerber, B., Aso, Y., and Yarali, A. (2018). Reinforcement signaling of punishment versus relief in fruit flies. Learn. Mem. 25, 247-257.

Kreyszig, E. (1979). Advanced Engineering Mathematics, Fourth Edition (Wiley).

Lee, T., and Luo, L. (1999). Mosaic analysis with a repressible cell marker fo studies of gene function in neuronal morphogenesis. Neuron 22, 451-461.

Lee, T., Lee, A., and Luo, L. (1999). Development of the Drosophila mushroom bodies: sequential generation of three distinct types of neurons from a neuroblast. Development 126, 4065-4076.

Lin, A.C., Bygrave, A.M., de Calignon, A., Lee, T., and Miesenböck, G. (2014). Sparse, decorrelated odor coding in the mushroom body enhances learned odor discrimination. Nat. Neurosci. 17, 559-568.

Litwin-Kumar, A., Oswald, A.M., Urban, N.N., and Doiron, B. (2011). Balanced synaptic input shapes the correlation between neural spike trains. PLoS Comput. Biol. 7, e1002305.

Liu, C., Plaçais, P.Y., Yamagata, N., Pfeiffer, B.D., Aso, Y., Friedrich, A.B. Siwanowicz, I., Rubin, G.M., Preat, T., and Tanimoto, H. (2012). A subset of dopamine neurons signals reward for odour memory in Drosophila. Nature 488, 512-516.

Liu, Q., Yang, X., Tian, J., Gao, Z., Wang, M., Li, Y., and Guo, A. (2016). Gap junction networks in mushroom bodies participate in visual learning and memory in Drosophita. eLife 5, e13238.

Luo, S.X., Axel, R., and Abbott, L.F. (2010). Generating sparse and selective third-order responses in the olfactory system of the fly. Proc. Natl. Acad. Sci. USA 107, 10713-10718.

Maiellaro, I., Lohse, M.J., Kittel, R.J., and Calebiro, D. (2016). CAMP signals in Drosophila motor neurons are confined to single synaptic boutons. Cell Rep. 17, 1238-1246.

Mao, Z., and Davis, R.L. (2009). Eight different types of dopaminergic neurons innervate the Drosophita mushroom body neuropil: anatomical and physiological heterogeneity. Front. Neural Circuits 3, 5 .

Martin, S.J., Grimwood, P.D., and Morris, R.G. (2000). Synaptic plasticity and memory: an evaluation of the hypothesis. Annu. Rev. Neurosci. 23, 649-711. McGuire, S.E., Le, P.T., and Davis, R.L. (2001). The role of Drosophila mushroom body signaling in olfactory memory. Science 293, 1330-1333.

McGuire, S.E., Le, P.T., Osborn, A.J., Matsumoto, K., and Davis, R.L. (2003). Spatiotemporal rescue of memory dysfunction in Drosophita. Science 302 , $1765-1768$.

Murtagh, F., and Contreras, P. (2017). Algorithms for hierarchical clustering: an overview, II. Wiley Interdiscip. Rev. Data Min. Know. Discov. 7, e1219.

Murthy, M., Fiete, I., and Laurent, G. (2008). Testing odor response stereotypy in the Drosophila mushroom body. Neuron 59, 1009-1023.

Niewalda, T., Völler, T., Eschbach, C., Ehmer, J., Chou, W.C., Timme, M., Fiala, A., and Gerber, B. (2011). A combined perceptual, physico-chemical, and imaging approach to 'odour-distances' suggests a categorizing function of the Drosophila antennal lobe. PLoS ONE 6, e24300.

Nighorn, A., Healy, M.J., and Davis, R.L. (1991). The cyclic AMP phosphodiesterase encoded by the Drosophila dunce gene is concentrated in the mush room body neuropil. Neuron $6,455-467$.

Niven, J.E., Anderson, J.C., and Laughlin, S.B. (2007). Fly photoreceptors demonstrate energy-information trade-offs in neural coding. PLoS Biol. 5, e116. 
Owald, D., Felsenberg, J., Talbot, C.B., Das, G., Perisse, E., Huetteroth, W., and Waddell, S. (2015). Activity of defined mushroom body output neurons underlies learned olfactory behavior in Drosophita. Neuron 86, 417-427.

Owald, D., and Waddell, S. (2015). Olfactory learning skews mushroom body output pathways to steer behavioral choice in Drosophita. Curr. Opin. Neurobiol. 35, 178-184.

Pech, U., Pooryasin, A., Birman, S., and Fiala, A. (2013). Localization of the contacts between Kenyon cells and aminergic neurons in the Drosophila metanogaster brain using SplitGFP reconstitution. J. Comp. Neurol. 521, 3992-4026.

Pech, U., Revelo, N.H., Seitz, K.J., Rizzoli, S.O., and Fiala, A. (2015). Optical dissection of experience-dependent pre- and postsynaptic plasticity in the Drosophila brain. Cell Rep. 10, 2083-2095.

Perez-Orive, J., Mazor, O., Turner, G.C., Cassenaer, S., Wilson, R.I., and Laurent, G. (2002). Oscillations and sparsening of odor representations in the mushroom body. Science 297, 359-365.

Perisse, E., Owald, D., Barnstedt, O., Talbot, C.B., Huetteroth, W., and Waddell, S. (2016). Aversive learning and appetitive motivation toggle feed-forward inhibition in the Drosophila mushroom body. Neuron 90, 1086-1099.

Pernelle, G., Nicola, W., and Clopath, C. (2018). Gap junction plasticity as a mechanism to regulate network-wide oscillations. PLoS Comput. Biol. 14, e1006025.

Poo, M.M., Pignatelli, M., Ryan, T.J., Tonegawa, S., Bonhoeffer, T., Martin, K.C., Rudenko, A., Tsai, L.H., Tsien, R.W., Fishell, G., et al. (2016). What is memory? The present state of the engram. BMC Biol. 14, 40.

Pouille, F., McTavish, T.S., Hunter, L.E., Restrepo, D., and Schoppa, N.E. (2017). Intraglomerular gap junctions enhance interglomerular synchrony in a sparsely connected olfactory bulb network. J. Physiol. 595, 5965-5986.

Qin, H., Cressy, M., Li, W., Coravos, J.S., Izzi, S.A., and Dubnau, J. (2012). Gamma neurons mediate dopaminergic input during aversive olfactory memory formation in Drosophila. Curr. Biol. 22, 608-614.

Riemensperger, T., Völler, T., Stock, P., Buchner, E., and Fiala, A. (2005). Punishment prediction by dopaminergic neurons in Drosophila. Curr. Biol 15, 1953-1960.

Sakimoto, Y., Mizuno, J., Kida, H., Kamiya, Y., Ono, Y., and Mitsushima, D. (2019). Learning promotes subfield-specific synaptic diversity in hippocampal CA1 neurons. Cereb. Cortex 29, 2183-2195.

Schleyer, M., Fendt, M., Schuller, S., and Gerber, B. (2018). Associative learning of stimuli paired and unpaired with reinforcement: evaluating evidence from maggots, flies, bees, and rats. Front. Psychol. 9, 1494.

Schultz, W., Dayan, P., and Montague, P.R. (1997). A neural substrate of prediction and reward. Science 275, 1593-1599.

Schwaerzel, M., Heisenberg, M., and Zars, T. (2002). Extinction antagonizes olfactory memory at the subcellular level. Neuron 35, 951-960.

Schwaerzel, M., Monastirioti, M., Scholz, H., Friggi-Grelin, F., Birman, S., and Heisenberg, M. (2003). Dopamine and octopamine differentiate between aversive and appetitive olfactory memories in Drosophila. J. Neurosci. 23, 10495-10502.

Shaner, N.C., Campbell, R.E., Steinbach, P.A., Giepmans, B.N., Palmer, A.E., and Tsien, R.Y. (2004). Improved monomeric red, orange and yellow fluorescent proteins derived from Discosoma sp. red fluorescent protein. Nat. Biotechnol. 22, 1567-1572.

Shannon, C.E. (1949). The Mathematical Theory of Communication (University of Illinois Press).

Shearin, H.K., Macdonald, I.S., Spector, L.P., and Stowers, R.S. (2014). Hexameric GFP and mCherry reporters for the Drosophila GAL4, Q, and LexA transcription systems. Genetics 196, 951-960.
Sosulski, D.L., Bloom, M.L., Cutforth, T., Axel, R., and Datta, S.R. (2011). Distinct representations of olfactory information in different cortical centres. Nature 472, 213-216.

Stuart, G.J., and Spruston, N. (2015). Dendritic integration: 60 years of progress. Nat. Neurosci. 18, 1713-1721.

Takemura, S.Y., Aso, Y., Hige, T., Wong, A., Lu, Z., Xu, C.S., Rivlin, P.K., Hess, H., Zhao, T., Parag, T., et al. (2017). A connectome of a learning and memory center in the adult Drosophila brain. eLife 6, e26975.

Takeuchi, T., Duszkiewicz, A.J., and Morris, R.G. (2013). The synaptic plasticity and memory hypothesis: encoding, storage and persistence. Philos. Trans. R. Soc. Lond. B Biol. Sci. 369, 20130288.

Tanaka, N.K., Awasaki, T., Shimada, T., and Ito, K. (2004). Integration of chemosensory pathways in the Drosophila second-order olfactory centers. Curr. Biol. 14, 449-457.

Tanimoto, H., Heisenberg, M., and Gerber, B. (2004). Experimental psychology: event timing turns punishment to reward. Nature 430, 983.

Technau, G.M. (1984). Fiber number in the mushroom bodies of adult Drosophila melanogaster depends on age, sex and experience. J. Neurogenet. 1, 113-126.

Tempel, B.L., Bonini, N., Dawson, D.R., and Quinn, W.G. (1983). Reward learning in normal and mutant Drosophita. Proc. Natl. Acad. Sci. USA 80 , 1482-1486.

Thévenaz, P., Ruttimann, U.E., and Unser, M. (1998). A pyramid approach to subpixel registration based on intensity. IEEE Trans. Image Process. 7, 27-41. Tian, L., Hires, S.A., Mao, T., Huber, D., Chiappe, M.E., Chalasani, S.H., Petreanu, L., Akerboom, J., McKinney, S.A., Schreiter, E.R., et al. (2009) Imaging neural activity in worms, flies and mice with improved GCaMP calcium indicators. Nat. Methods 6, 875-881.

Tomchik, S.M., and Davis, R.L. (2009). Dynamics of learning-related CAMP signaling and stimulus integration in the Drosophila olfactory pathway. Neuron 64, 510-521.

Tully, T., and Quinn, W.G. (1985). Classical conditioning and retention in normal and mutant Drosophila melanogaster. J. Comp. Physiol. A Neuroethol. Sens. Neural Behav. Physiol. 157, 263-277.

Turner, G.C., Bazhenov, M., and Laurent, G. (2008). Olfactory representations by Drosophila mushroom body neurons. J. Neurophysiol. 99, 734-746.

Vosshall, L.B., and Stocker, R.F. (2007). Molecular architecture of smell and taste in Drosophila. Annu. Rev. Neurosci. 30, 505-533.

Wagh, D.A., Rasse, T.M., Asan, E., Hofbauer, A., Schwenkert, I., Dürrbeck, H., Buchner, S., Dabauvalle, M.C., Schmidt, M., Qin, G., et al. (2006). Bruchpilot, a protein with homology to ELKS/CAST, is required for structural integrity and function of synaptic active zones in Drosophila. Neuron 49, 833-844.

Wise, R.A. (2004). Dopamine, learning and motivation. Nat. Rev. Neurosci. 5 , 483-494.

Yamagata, N., Hiroi, M., Kondo, S., Abe, A., and Tanimoto, H. (2016). Suppression of dopamine neurons mediates reward. PLoS Biol. 14, e1002586.

Yamazaki, D., Hiroi, M., Abe, T., Shimizu, K., Minami-Ohtsubo, M., Maeyama, Y., Horiuchi, J., and Tabata, T. (2018). Two parallel pathways assign opposing odor valences during Drosophila memory formation. Cell Rep. 22, 2346-2358. Yarali, A., Nehrkorn, J., Tanimoto, H., and Herz, A.V.M. (2012). Event timing in associative learning: from biochemical reaction dynamics to behavioural observations. PLOS ONE 7, e32885.

Yuan, Q., Lin, F., Zheng, X., and Sehgal, A. (2005). Serotonin modulates circadian entrainment in Drosophila. Neuron 47, 115-127.

Zars, T., Fischer, M., Schulz, R., and Heisenberg, M. (2000). Localization of a short-term memory in Drosophita. Science 288, 672-675. 
Neuron

\section{Article}

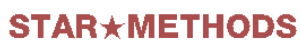

KEY RESOURCES TABLE

\begin{tabular}{|c|c|c|}
\hline REAGENT or RESOURCE & SOURCE & IDENTIFIER \\
\hline \multicolumn{3}{|l|}{ Antibodies } \\
\hline Mouse anti-discs large (DLG) & Developmental Studies Hybridoma Bank & 4F3; RRID: AB_528203 \\
\hline Rabbit anti-GFP & Invitrogen & A6455; RRID: AB_221570 \\
\hline Mouse anti-IgG (Alexa Fluor 633) & Invitrogen & A21050; RRID: AB_2535718 \\
\hline Rabbit anti-lgG (Alexa Fluor 488) & Life Technologies & A11034; RRID: AB_2576217 \\
\hline \multicolumn{3}{|l|}{ Chemicals, Peptides, and Recombinant Proteins } \\
\hline $\begin{array}{l}\text { Ringer's solution ( } 5 \mathrm{mM} \mathrm{KCl}, 130 \mathrm{mM} \mathrm{NaCl} \text {, } \\
2 \mathrm{mM}, \mathrm{MgCl}_{2}{ }^{*} 2 \mathrm{H}_{2} \mathrm{O}, 2 \mathrm{mM} \mathrm{CaCl}, 5 \mathrm{mM} \\
\text { HEPES, } 36 \mathrm{mM} \text { sucrose, } \mathrm{pH} 7.3)\end{array}$ & $\mathrm{N} / \mathrm{A}$ & N/A \\
\hline $\begin{array}{l}\text { Phosphate buffered saline (PBS; } 15 \mathrm{mM} \\
\mathrm{NaH}_{2} \mathrm{PO}_{4}, 100 \mathrm{mM} \mathrm{NaCl}, 85 \mathrm{mM} \mathrm{Na} \mathrm{HPO}_{4} \text { ) }\end{array}$ & N/A & N/A \\
\hline Triton $\mathrm{X}-1000$ (used at $0.6 \%$ in PBS) & Carl Roth & 3051.1 \\
\hline $\begin{array}{l}\text { Bovine serum albumin (used at } 2 \% \text { in PBST } \\
\text { solution) }\end{array}$ & Carl Roth & 8076.2 \\
\hline $\begin{array}{l}\text { Paraformaldehyde (used at } 4 \% \text { in PBS with } \\
0.1 \% \mathrm{NaOH} \text { ) }\end{array}$ & Carl Roth & 03435.1 \\
\hline Mineral oil & Sigma-Aldrich & M8410 \\
\hline 4-Methylcyclohexanol (MCH) & Sigma-Aldrich & 153095 \\
\hline 3-octanol (3-oct) & Sigma-Aldrich & 218405 \\
\hline 1-octanol (1-oct) & Sigma-Aldrich & 297887 \\
\hline \multicolumn{3}{|l|}{ Deposited Data } \\
\hline $\begin{array}{l}\text { All } \mathrm{Ca}^{2+} \text { imaging data and their derivatives } \\
\text { are deposited in Database: Mendeley Data } \\
\text { https://data.mendeley.com/datasets/ } \\
\text { wf/gz3wfr3/draft?a=aabec5a9-b066- } \\
\text { 4994-a3ca-b2e692cd92fo }\end{array}$ & Mendeley Data & $\begin{array}{l}\text { https://doi.org/10.17632/ } \\
\text { wf7gz3wfr3.1 }\end{array}$ \\
\hline \multicolumn{3}{|l|}{ Experimental Models: Organisms/Strains } \\
\hline $\mathrm{y}^{1}, \mathrm{w}^{1118}$, P\{neoFRT $\} 19 \mathrm{~A} ;+;+$ & Bloomington Stock Centre & 1744 \\
\hline $\begin{array}{l}\text { w }^{\star}, \text { P\{hsFLP\}1, P\{tubP-GAL80\}LL1, P } \\
\{\text { neoFRT\}19A; +; + }\end{array}$ & Bloomington Stock Centre & 5132 \\
\hline$w^{1118} ;+;$ P $\{$ UAS-brp.S-EGFP $\}$ & Fouquet et al., 2009 & N/A \\
\hline $\begin{array}{l}y^{1}, w^{\star} ; \text { PBac }\{20 X U A S-6 X m C h e r r y-H A\} \\
\text { VK00018/CyO, P\{Wee-P.ph0\}Bacc[Wee- } \\
\text { P20]; Dr } \\
{ }^{1} / \mathrm{TM} 6 \mathrm{C}\end{array}$ & Bloomington Stock Centre & 52267 \\
\hline $\begin{array}{l}y^{1}, w^{\star} ; \text { Sp/CyO, P\{Wee-P.pho\}Bacc[Wee- } \\
\text { P20] ; P\{20XUAS-6XmCherry-HA\}attP2; }\end{array}$ & Bloomington Stock Centre & 52268 \\
\hline $\mathrm{W}^{*} ;+;$ P\{GAL4-5-HTR1B.Y $\} 3$ & Bloomington Stock Centre & 27637 \\
\hline $\begin{array}{l}w^{1118} ;+; \text { PBac }\{20 X U A S-G C a M P 3\} \\
\text { VK00005 }\end{array}$ & Bloomington Stock Centre & 32237 \\
\hline $\mathrm{W}^{\star} ; \mathrm{P}\{20 \mathrm{XUAS}-\mathrm{GCaMP} 3\} ;+$ & Tian et al., 2009 & N/A \\
\hline$w^{1118} ;+;$ P $\{5 X U A S-S y p-G C a M P 3\} ;$ & Pech et al., 2015 & N/A \\
\hline \multicolumn{3}{|l|}{ Software and Algorithms } \\
\hline ImageJ/FIJI & National Institutes of Health & https://imagej.net/Fiji \\
\hline Zen 2011 SP2 & Carl Zeiss AG & N/A \\
\hline Leica Application Suite X (LAS) & Leica Microsystems GmbH & $\mathrm{N} / \mathrm{A}$ \\
\hline
\end{tabular}

(Continued on next page) 


\begin{tabular}{lll}
\hline Continued & & \\
\hline REAGENT or RESOURCE & SOURCE & IDENTIFIER \\
\hline MATLAB (R2018) & MathWorks & N/A \\
False Discovery Rate Detection & Benjamini and Hochberg, 1995 https://de.mathworks.com/matlabcentral/ \\
& & fileexchange/27418-fdr_bh \\
$\begin{array}{l}\text { ACC Index Scripts (MATLAB), Database: } \\
\text { GitHub }\end{array}$ & This paper & https://github.com/zerotonin/ \\
\hline
\end{tabular}

\section{RESOURCE AVAILABILITY}

Lead Contact

Further information and requests for resources and reagents should be directed to and will be fulfilled by the Lead Contact, André Fiala (afiala@gwdg.de).

Materials Availability

All unique/stable reagents, as well as fly strains generated in this study are available from the Lead Contact without restriction.

\section{EXPERIMENTAL MODEL AND SUBJECT DETAILS}

\section{Drosophila melanogaster}

The following Drosophila strains were used and genetically combined: Hsp70-FLP1, neoFRT19A, tubP-Gal80 (Lee and Luo, 1999; Bloomington stock \#5132), neoFRT19A (Bloomington stock \#1744), 20xUAS-6xmCherry (Shearin et al., 2014; Bloomington stocks \#52267 and \#52268), 20xUAS-GCaMP3 (Tian et al., 2009), UAS-Syp-GCaMP3 (Pech et al., 2015), and 5-HT1B-Gal4 (Yuan et al., 2005; Bloomington stock \#27637). Flies were maintained on standard cornmeal medium at $25^{\circ} \mathrm{C}, 60 \%$ humidity, and on a $12: 12-$ hour light-dark cycle. For all experiments, female flies aged 3-6 days after eclosion were used.

\section{METHOD DETAILS}

MARCM-mediated generation of single-cell clones

Virgin female flies carrying the Gal4-driver (5-HT1B-Gal4) and the neoFRT19A inserts (both homozygous) were crossed with male flies carrying the X chromosome neoFRT19A and the Hsp70-FLP1, tubP-Gal80 inserts, 20xUAS-6xmCherry balanced over CyO, and 20xUAS-GCaMP3 balanced over TM6. For imaging using Syp-GCaMP, the strain 5xUAS-Syp-GCaMP3 balanced over CyO and the strain 20xUAS-6xmCherry balanced over TM6 were used. Female flies were allowed to lay eggs for a 4-hour period. After a 48-hour interval the larvae were heat-shocked twice in a $37^{\circ} \mathrm{C}$ water bath; each heat shock was of 45 minutes duration, with a 30-minute interval between treatments in order to induce FLP activity during the development of $\gamma$-lobe KCs.

Optical $\mathrm{Ca}^{2+}$ imaging

Female flies (3-6 days old) were placed in a custom-built shock-delivery chamber. Their heads were fixed using UV-hardening dental glue (Kentoflow, Kent Dental, 953 683, A2), and their head capsules were opened and covered with a droplet of Ringer's solution. Fluorescence changes in the boutons of single KCs were monitored at a frame rate of $4 \mathrm{~Hz}$ using an LSM 7MP 2-photon microscope (Zeiss, Oberkochen, Germany) equipped with a mode-locked Ti-sapphire laser (Coherent, Santa Clara, CA, USA) and a Plan-Apochromat 20x (NA = 1) water immersion objective. Both sensors were excited at $940 \mathrm{~nm}$, and a dichroic mirror was combined with 500 to $550 \mathrm{~nm}$ and 650 to $660 \mathrm{~nm}$ BP filters to simultaneously record GCaMP and mCherry. A custom-built odor stimulation device was attached to the microscope imaging chamber and used to present the stimulus, consisting of mineral oil solvent (Sigma-Aldrich, Saint Louis, MO, USA, \#M8410), 4-methylcyclohexanol (1:750 dilution; Aldrich Chemistry, Milwaukee, WI, USA \#153095), 3-octanol (1:500 dilution; Aldrich Chemistry \#218405), or 1-octanol (1:400 dilution, Aldrich Chemistry \#297887) to the fly antennae at a flow rate of $1 \mathrm{~mL} / \mathrm{s}$. The initiation and duration of the odor stimuli were controlled using in-house LabVIEW software (National Instruments, Austin, TX, USA). Depending on the complexity of the single KCs, 3-7 focal planes (median 5) were imaged in order to capture as much of the axons as possible. For most experiments cytosolic GCaMP3 was used because of its higher baseline fluorescence and localization along the entire axon when compared with Syp-GcaMP3. This facilitated the recognition/identification of axonal branches and synaptic boutons across imaging trials. Only KCs that showed robust odor-evoked responses exceeding three standard deviations of the pre-stimulus baseline were considered responsive. 
Odor stimulation and olfactory conditioning

To visualize $\mathrm{Ca}^{2+}$ dynamics in synaptic boutons evoked by different odorants, one imaging measurement was taken for each odorant for a duration of $21.25 \mathrm{~s}$ in each focal plane, with the onset of odor presentation $6.25 \mathrm{~s}$ after the onset of the measurement. Each odor was presented for $2.5 \mathrm{~s}$, and there was a $20 \mathrm{~s}$ interval between each measurement. After all odorants had been measured in one focal plane and after all necessary focal planes (3-7) had been measured, the associative learning protocol was initiated. First, the odorant used as CS+ (4-methylcyclohexanol or 3-octanol), was presented $10 \mathrm{~s}$ after onset of the measurement for a duration of $60 \mathrm{~s}$. Five seconds after odor onset, 12 electric shocks ( $90 \mathrm{~V}, 1.25 \mathrm{~s}$ duration, with a $3.75 \mathrm{~s}$ rest period between shocks) were temporally paired with the first presented odor (CS+). After a resting period of $60 \mathrm{~s}$, the second odor (again, 4-methylcyclohexanol or 3-octanol, depending on which odorant was used as CS+) was presented without an electric shock (CS-) for $60 \mathrm{~s}$. The imaging continued for $30 \mathrm{~s}$ after odor offset. The control group received the same stimulation protocol but without the electric shock. After training, the flies were given a 3-minute resting period. Odor-evoked $\mathrm{Ca}^{2+}$ transients were then measured in all focal planes, similar to before the associative learning protocol. The recorded images were processed with Image $/$ software (NIH, Bethesda, MD, USA) and movements of in-plane direction were corrected using custom-written plugins, employing turboReg (Thévenaz et al., 1998) and Kalman filtering (ImageJ plugin by Christopher P. Mauer https://imagej. net/plugins/kalman.html). The $\Delta \mathrm{F} / \mathrm{F}_{0}$ was calculated for each identified bouton in each KC for both the GCaMP and mCherry sensors. $F_{0}$ was calculated by taking the average pixel intensity of 20 frames, starting from the third frame after the onset of recording. $\Delta \mathrm{F}$ was calculated by taking the average of five frames around the peak fluorescence intensity after the onset of odor presentation and subtracting the $F_{0}$ for each individual bouton. A sliding average of three frames was used to reduce noise in the data. Additionally, the $\Delta F / F_{0}$ of mCherry was subtracted from the $\Delta F / F_{0}$ of GCaMP. All KC boutons were assigned to a $\gamma$-lobe compartment using data from immunohistochemical images.

Immunohistochemistry and confocal microscopy

Following removal of the flies from the imaging chamber, brains were dissected in ice-cold Ringer's solution and then fixed in $4 \%$ para-formaldehyde for 2 hours at $4^{\circ} \mathrm{C}$. Brains were rinsed three times for 20 minutes each in phosphate buffered saline (PBS) and subsequently incubated in blocking solution (PBS containing $0.6 \%$ Triton X-100 and $2 \%$ bovine serum albumin) for 2 hours at room temperature. Brains were then incubated with the primary antibodies (mouse anti-discs, large, Developmental Studies Hybridoma Bank 4F3, 1:200; and rabbit anti-GFP serum, Invitrogen A6455, 1:2000), diluted in blocking solution at $4^{\circ} \mathrm{C}$ overnight. Brains were then rinsed three times, for 20 minutes each time, in PBS containing $0.6 \%$ Triton X-100 at room temperature and then incubated in with secondary antibodies (Alexa Fluor 488 goat anti-rabbit IgG, Life Technologies, Carlsbad, CA, USA, A11034, 1:300; and Alexa Fluor 633 goat anti-mouse, Invitrogen, Carlsbad, CA, USA, A21050,1:300), diluted in blocking solution at $4^{\circ} \mathrm{C}$ overnight. The brains were then rinsed at room temperature, first in PBS containing $0.6 \%$ Triton X-100 for 20 minutes and then twice in PBS for 20 minutes. Finally, brains were embedded in Vectashield (Vector Laboratories, Burlingame, CA, USA) on microscope slides. Microscopy was conducted using a confocal laser scanning microscope (SP8, Leica Microsystems, Wetzlar, Germany) equipped with an HC PL APO 20x (NA = 0.75) water immersion objective. Image stacks were analyzed and reconstructed in three dimensions using ImageJ.

Agglomerative hierarchical clustering

To reduce the complexity of the $\mathrm{Ca}^{2+}$ signals of individual boutons we used an agglomerative hierarchical clustering approach. The agglomerative clustering algorithm segregates the continuum of complex responses into a smaller subset of reoccurring response patterns. We used the peak $\mathrm{Ca}^{2+}$ amplitude and the duration between stimulus onset and maximum $\mathrm{Ca}^{2+}$ response as the feature space. Because these values have different dimensions $\left(\Delta \mathrm{F} / \mathrm{F}_{0}, \mathrm{~s}\right)$ and could have different numerical ranges, we normalized the data to a mean of 0 and a standard deviation of 1 ( $z$-score) (Kreyszig, 1979). Differences between clusters were then calculated as their Euclidean distance in the $z$-scored parameters (peak amplitude, time to peak). Each bouton $\mathrm{Ca}^{2+}$ response was treated initially as asingle cluster; these single clusters were then merged so that Ward's method (Murtagh and Contreras, 2017) was fulfilled. Ward's criterion (Murtagh and Contreras, 2017) is to minimize the variance of the new merged cluster. The algorithm tries to find the merger that would create the smallest variance in the new cluster. The variance of the merge is saved as the cost of clustering (see Figure $7 \mathrm{~A}$ ). All boutons are merged until all are unified in one cluster. The cost function of this clustering can then be inspected to find the point when native segregation of data is reached, which is signified by a steep increase in costs. We found a native segregation of four distinct clusters in all cases. We designated these clusters as BRCs, in which the cluster centroids formed the focal point for all responses assigned to those clusters. The cluster borders (Voronoi cells around the centroids) were used to categorize the conditions after training for CS+, CS-, and controls. Note that each bouton $\mathrm{Ca}^{2+}$ response was assigned to a resulting BRC and that the responses changed from a continuous representation into a quantized/categorized representation. Shannon entropy (Shannon, 1949) was used as a measure for the variance (Allaj, 2018) (i.e., the BRCs of $\gamma$ lobe compartments) because classical variance measures are inappropriate for categorical data.

Amplitude-corrected correlation analysis

We developed a simple but robust technique to quantify the congruence as a measure of "similarity" of $\mathrm{Ca}^{2+}$ transients between synaptic boutons both within and across each $\gamma$-lobe compartment. The core of the analysis is a cross-correlation of the $\mathrm{Ca}^{2+}$ responses of two synaptic boutons; the cross-correlation is normalized by its auto-correlation, resulting in 1 if both responses are identical. Such a calculation was made for each bouton, and the median ACC per $\gamma$-lobe compartment is shown. Slight movements of the brain can return false positive results, as they can result in changes in fluorescence that are highly synchronous across all com- 
partments. To correct for these false positives, the mean response amplitude and the complement of the Michelson contrast between both amplitudes were factored into the correlation coefficient (Equation 1),

$$
A C C=\frac{\int C l_{1}^{*}(\tau) C_{2}(t+\tau) \delta \tau}{\sqrt{\sum_{\alpha \varepsilon C l_{1}} \mathrm{a}^{2}} \cdot \sqrt{\sum_{\beta \varepsilon C C_{2}} b^{2}}} \cdot \frac{\frac{A_{1}+A_{2}}{2}}{\max \left(A_{1 \rightarrow n}\right)} \cdot\left(1-\frac{A_{1}-A_{2}}{A_{1}+A_{2}}\right),
$$

(Equation 1)

where $\mathrm{ACC}$ is the amplitude-corrected correlation index, $\mathrm{Cl}_{1}$ and $\mathrm{Cl}_{2}$ are two bouton $\mathrm{Ca}^{2+}$ response time series, $t$ is the time point of the response, and $\tau$ is the phase lag of the correlation. $A_{1}$ and $A_{2}$ are the respective maximum amplitudes of $\mathrm{Cl}_{1}$ and $\mathrm{Cl}_{2} . \alpha$ and $\beta$ denote each sample of the respective response, and $n$ is the entirety of all bouton response amplitudes of one cell. All three terms return a number in the range 0 to 1 , and their product is exactly unity if both signals are identical. The ACC is 0 if either amplitude of the two boutons is 0 (one cell not responding) or if the $\mathrm{Ca}^{2+}$ signals are not temporally correlated.

To assess the internal correlation of boutons within a single $\gamma$-lobe compartment, we calculated the median ACC of all possible bouton combinations within each compartment $\left(A C C_{\text {intra }}\right)$. The intercompartment $A C C\left(A C C_{\text {inter }}\right)$ was calculated similarly by using only combinations of boutons from a single compartment to boutons of other compartments (see Figure 2D). A Michelson contrast of these two ACCs (see Equation 2) (ACC index contrast, $A C C_{C}$ ) determined if the $\gamma$-lobe was more similar to itself (positive value maximum $=1.0$ ) or the other $\gamma$-lobes (negative values) (Figure 2E). Significance was determined by calculating the $95 \%$ confidence interval $(\mathrm{Cl})$ of the median over cells. If the $\mathrm{Cl}$ did not include 0 , the $\mathrm{Ca}^{2+}$ transients of boutons within a single compartment were considered to be significantly more correlated to themselves than to those of other lobe compartments $(p<0.05)$.

$$
A C C_{c}=\frac{A C C_{\text {intra }}-\overline{A C C_{\text {inter }}}}{A C C_{\text {intra }}+\overline{A C C_{\text {inter }}}}
$$

(Equation 2)

The effect of associative learning on the congruence of the different $\mathrm{KC} \gamma$-lobe compartments was determined by subtracting the pretraining ACC for each $\gamma$-lobe compartment from the posttraining ACC. We calculated the differences for each cell, and individually for the control, CS-, and CS+ conditions. We again used the $95 \% \mathrm{Cl}$ of the median across cells to test for significance. If the $\mathrm{Cl}$ did not include 0 , we concluded that the $\gamma$-lobe compartment had significantly changed its congruence $(p<0.05)$. All scripts used to calculate the ACC are available at https://github.com/zerotonin/KCC_KenyonCellCorrelator.

\section{QUANTIFICATION AND STATISTICAL ANALYSIS}

Statistical details for each experiment, including $n$ numbers and the statistical test performed, can be found in the corresponding figure legends. $\mathrm{Ca}^{2+}$ traces across cells or boutons represent mean \pm standard deviation (Figures $3 \mathrm{~A}$ and $5 \mathrm{C}$ ) or, in case of showing response classes, median $\pm 95 \%$ confidence interval (Figure 7B). All boxplots show the median as the central value, notches show $95 \%$ confidence intervals, and boxes represent $2^{\text {nd }}$ and $3^{\text {rd }}$ quartiles (Figures $4 \mathrm{~A}, 6 \mathrm{~A}, 7 \mathrm{E}$, and $7 \mathrm{G}$ ). Black squares in Figures 4A, 6A, and $\mathrm{S} 4$ indicate means. Whiskers show full data ranges, except if outliers occur, in which cases whiskers represent $1.5 \mathrm{x}$ interquartile ranges. In all other plots central values represent median, and dispersion is represented as the $95 \%$ confidence interval of the median.

Throughout the manuscript, we employ non-parametric tests, i.e., Kruskal-Wallis test (Figure 4A), sign test (Figure 6A), exact statistics as median differences (Figures 4E, 6B, S5, and S6B), Fisher's permutation test (Figure 5C), and $\mathrm{X}^{2}$-test (Figure 7D). Hence we made no assumptions about the distribution of the data. Test types and $p$ values are noted in the corresponding figure legends. When more than one comparison was made we corrected $p$ values using the Benjamini-Hochberg false-discovery-rate algorithm (Benjamini and Hochberg, 1995; implementation in MATLAB by David M. Groppe (https://de.mathworks.com/matlabcentral/fileexchange/ 27418-fdr_bh)). All statistical calculations were performed in MATLAB R2018 (Mathworks, Natwick, USA).

\section{DATA AND CODE AVAILABILITY}

The accession number for the data reported in this paper is: [Mendeley Data]: [doi:10.17632/wf7gz3wfr3.1] and [GitHub]: [https:// github.com/zerotonin/KCC_KenyonCellCorrelator]. 


\subsection{Discussion}

With this study, we presented a novel mechanism by which the mushroom body (MB) encodes odours. This was possible by monitoring odour-evoked activity in single MB Kenyon cells (KCs) at the level of presynaptic bouton structures along the axons. This allowed for the examination of this activity in the context of the compartmentalised structure of the $\mathrm{MB} Y$-lobe within the same neuron. Therein, we were able to show that odour-evoked activity in the KCs is not homogenous along the axon, but rather that it is heavily influenced by the differential innervation patterns along the axons.

Furthermore, the implementation of a new metric for the quantification of congruence between synaptic boutons - the so-called Amplitude Corrected Correlation (ACC), introduced here demonstrated that the pattern of activity across synaptic boutons may in fact be informative of the learned value of an odour. Indeed, after conditioning flies with an odour-electric shock pairing protocol, the representation of the trained odour became more decorrelated across boutons. Therein, the degree to which synaptic boutons respond in concert with one another is identified as a novel, quantifiable characteristic of an odour's neural representation.

The implication of this decorrelation was then examined in the context of information theory. Upon finding that all bouton responses fell into one of four "bouton response classes" via hierarchical clustering analysis, this categorisation allowed for the calculation of the estimated bit-wise odour coding capacity of the MB g-lobe under different experimental conditions. That is, by calculating the distribution of all bouton responses across those response classes, we used Shannon's information theory to infer how this relates to the potential storage of new information (i.e., that an odour is predictive of punishment) across the population of boutons. Indeed, learning-induced decorrelation coincided with a broader distribution of responses across classes, which translated to a greater bitwise coding capacity after associative learning.

This study highlighted the importance of individual boutons, more so than the neuron as a whole, as odour coding entities that can be individually modulated through olfactory conditioning. The downstream implications of such a odour coding mechanism are not currently clear, but it would seem intuitive that more cohesive synaptic bouton activity would lead to a more robust input signal to postsynaptic partners (such as the mushroom body output neurons). The mechanism by which the boutons maintain their functional individuality it also not clear, although previous studies have implicated the phosphodiesterase encoded by the dunce gene in the spatial restriction of CAMP and PKA signalling in the MB (Gervasi et al., 2010), and it appears to localise to presynaptic release sites in other model systems (Charlie et al., 2006). 
In the context of the previous study of the MBONs (section 4), in which we identified associative effects only in the $\gamma 1$-innervating MBON (Figure 4.3.), it is of importance to note that recordings from the $\gamma 1$ region at the level of the $K C$ boutons was not experimentally possible here. Due to the position of this compartment in the brain, the functional imaging procedure would have to be adapted such that the $\gamma 1$ can be monitored as well as the $\gamma 2-\gamma 5$ compartments. In light of the findings presented in section 4 , future investigation of the $\gamma 1$ alone would be beneficial to elucidate the presynaptic modulation that occurs in parallel with the specific suppression of MBON- $\gamma 1$ after associative conditioning. 


\section{Functional and Anatomical Investigation of MB-CP1 Neurons}

\subsection{Introduction and aims}

In this chapter, we move our focus away from the lobes of the mushroom body and to the calyx. In section 4, we discussed a specific population of mushroom body output neurons (MBONs) that lie directly downstream of the mushroom body intrinsic Kenyon cell (KCs) axons that form the $\mathrm{y}$-lobe. Here, we have begun to investigate a different type of $M B O N$ that receives synaptic input primarily in the calycal region of the mushroom body.

The circuitry of the mushroom body calyx, formed primarily by the dendrites of the KCs, incorporates inputs and outputs from a broad array of neuronal populations, facilitating connections between diverse brain regions and sensory modalities. The most numerous of the input synapses to the calyx are formed by olfactory projection neurons, the main postsynaptic partners of which are the mushroom body KCs (Li et al., 2020). Accordingly, as integrators of KC activity, the MBONs that innervate the lobe regions of the mushroom body display excitatory responses to most experimental odorants (Hige, Aso, Rubin, et al., 2015). This is a characteristic that, notably, distinguishes the calyxinnervating MBONs (MB-CP1) from the majority of the MBON population.

A previous study revealed that the MB-CP1 neurons display a very narrow odour response profile, whereby robust responses were elicited only by the smells of vinegar and yeast (Hige, Aso, Rubin, et al., 2015). This implies that, despite the dense dendritic arbours of the MB-CP1 neurons in a region characterised by strong olfactory input, only few functional connections are formed with MP-CP1 neurons. Furthermore, the fact that these connections seem to be specific for olfactory stimuli related to desirable food odours invokes questions regarding the potential role of these neurons in the direction of innate, food-related behaviours.

In this study, following a detailed characterisation of the anatomy of the MB-CP1 neurons and finding they have no role in learning and memory, we aimed primarily to investigate their functional properties in the context of olfactory processing. We utilise the in vivo calcium imaging techniques described in previous sections to expose flies to a panel of odorants under different conditions with the goal of elucidating the factors influencing the odour tuning profile of the MB-CP1 neurons. These experiments provide the foundation for a more in-depth investigation of these neurons in the future. 


\subsection{Results}

\subsubsection{Anatomical characterisation of $\mathrm{MB}-\mathrm{CP} 1$}

While the MBONs described in section 4 were characterised by their innervation of the lobes of the mushroom body, the MBONs that we explore here are distinct in their innervation of the calycal region of the MB. This means that these neurons are positioned to receive input at the level of the Kenyon cell dendrites, rather than the axonal compartments. We first sought to further describe these anatomical properties of these MBONs (MB-CP1).

By expressing a GFP reporter under the control of MB-CP1-specific drivers, we could visualise the neurons in explanted brains (Figure 7.1., A and B). This allowed us to visualise, in fine detail, their innervation pattern in the MB calyx. There, the MB-CP1 neurons extend a broad and comprehensive mass of dendritic arbours throughout (Figure 7.1., A and B). Two split-gal4 driver lines were used here - MB622B and MB242A. The former results in reporter expression in a single MB-CP1 neuron per hemisphere (Figure 7.1., A, C, and D), while the latter results in expression in two MB-CP1 neurons (Figure 7.1., B, E, and F). These expression patterns can also be seen in the larval stage (Figure 7.1., CF).
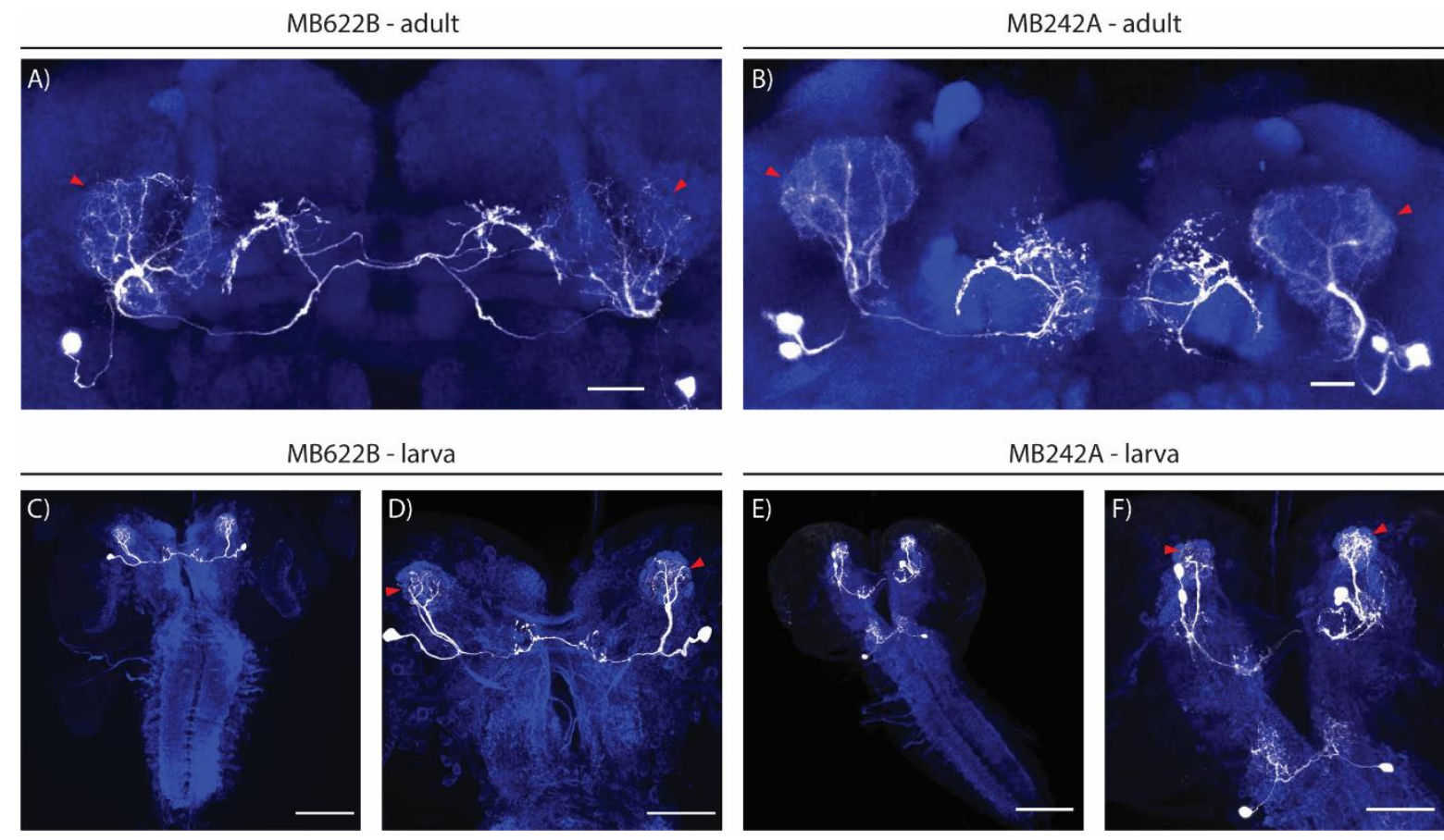

Figure 7.1.: Anatomical characterisation of MB-CP1 neurons. (A) and (B) Expression of mCD8-GFP and nsyb-GFP under the control of the two split gal4 driver lines used in the study - MB622B (A) drives expression in a single $M B-C P 1$ neuron per hemisphere, while $M B 242 A(B)$ drives expression in the same MB-CP1 neuron as MB622B and another additional MB-CP1 neuron with very similar anatomical features. MB-CP1 neuron dendrites densely innervate the calyx of the MB (red arrow heads) and through the pedunculus. The axons then extend both ipsilaterally and contralaterally in the crepine 
region surrounding the horizontal lobes of the MB. Scale bars $=20 \mu \mathrm{m}$. (C)-(F) The expression patterns of these drivers are also seen in the larval stage. (E) and (F) MB242A additionally drives expression in a single cell per hemisphere in the suboesophageal zone. Scale bars $=100 \mu \mathrm{m}$. Larval dissection and immunohistochemistry carried out by Maria Woitow.

Innervation of the MB calyx implies a connectedness with the MB-intrinsic Kenyon cells. Thus, this putative connection was also visualised using a split-GFP approach, wherein we generated flies in which subunits 1-10 of GFP were expressed in MB-CP1 neurons and the last subunit expressed in the MB Kenyon cells. The resultant reconstituted GFP signal can be seen in Figure 7.2., where the presence of GFP signal indicates sites at which the divided GFP subunits were in close enough proximity to emit fluorescence. With this tool, we could infer the possibility of connection between the MB-CP1 neurons and the MB through the majority of the volume of the calyx (Figure 7.2., A-C), as well as in the horizontal lobes, where the axons of the MB-CP1 neurons projection (Figure 7.2., D-F). Indeed, these connections are also supported by a recent MB connectomics study (Li et al., 2020).

Given the extensive olfactory input to the MB calyx (via olfactory projection neurons), these structural properties also appear to imply the possibility of a broad olfactory input to the MB-CP1 neurons described here. However, this does not seem to be reflected in the functional properties of these cells, as will be discussed later in section 7.2.3.
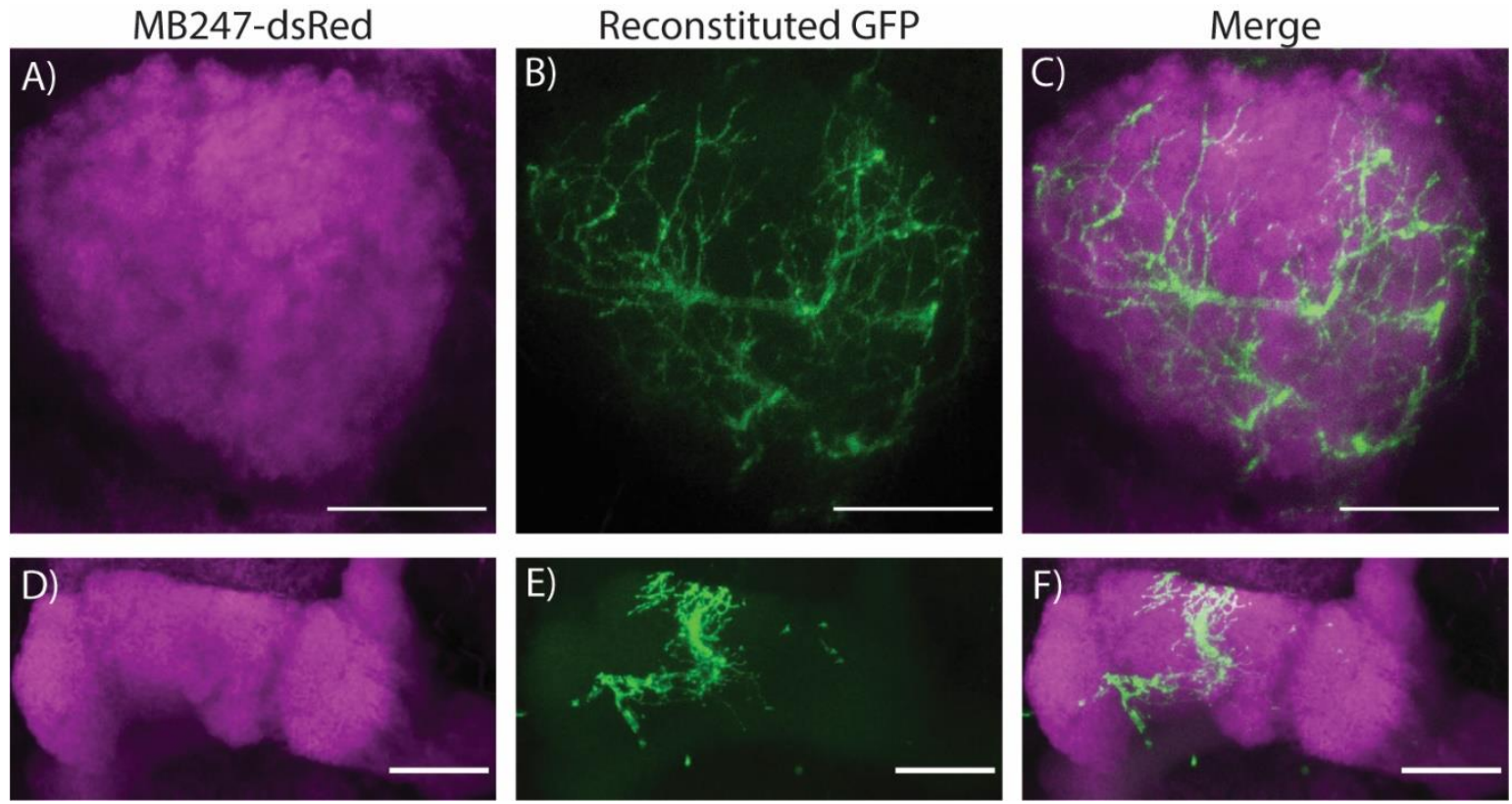

Figure 7.2.: Visualisation of potential regions of $\mathrm{MB}-\mathrm{CP} 1$ neuron-MB interaction. Split-GFP reconstitution between $\mathrm{MB}-\mathrm{CP} 1$ neurons and $\mathrm{MB} K \mathrm{KC}$ in the calyx (A-C) and horizontal lobe (D-F) of the MB. MB visualised by genetic expression of dsRed in KCs (under control of the MB247 promoter). Scale bars $=20 \mu \mathrm{m}$. 


\subsubsection{MB-CP1 neuron output is not required for learning and short-term memory}

Many MBONs have been identified as playing a role in associative olfactory conditioning (Aso et al., 2014b; Owald et al., 2015; Perisse et al., 2016). Therefore, we wanted to test if the synaptic output of the MB-CP1 neurons is also required for the formation and expression of either aversive or appetitive short-term associative memories. To test this, we utilised the Gal4-UAS system to express the temperature-sensitive dynamin transgene, shibire ${ }^{t s}$ (Kitamoto, 2001), in the MB-CP1 neuron(s). By conducting experiments at a restrictive temperature of $32^{\circ} \mathrm{C}$, we were able to cause a depletion of the ready-releasable vesicle pool in these neurons such that synaptic output was temporarily inhibited.

We tested the impact of MB-CP1 neuron output inhibition on both aversive (odour-electric shock pairing) and appetitive (odour-sucrose pairing) associative olfactory conditioning and found no reduction in the learning scores in either case, using either driver line (Figure 7.3.). We conclude, therefore, that there is no substantial role of the MB-CP1 neurons in short-term learning or memory formation.

A)

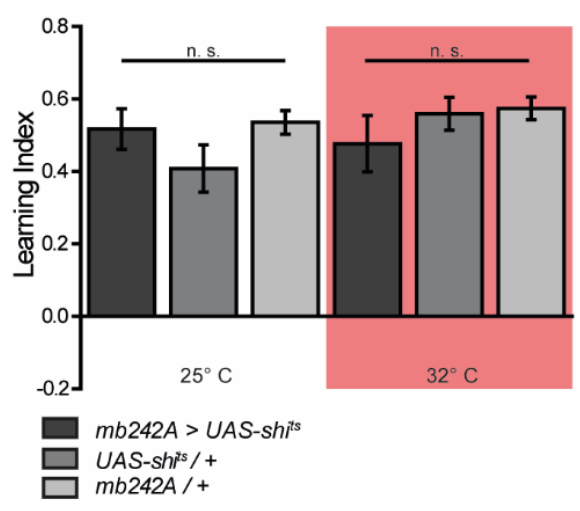

C)

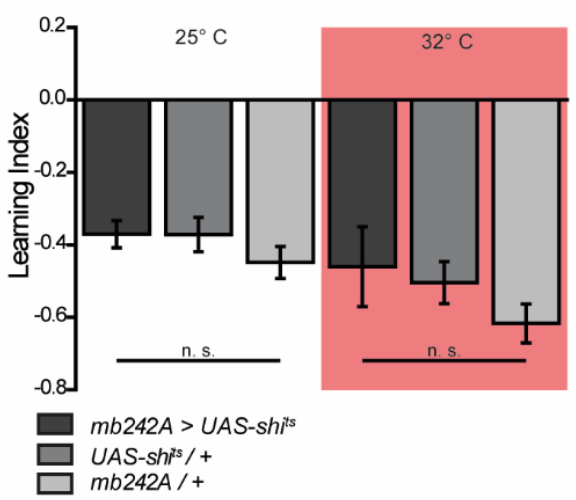

B)

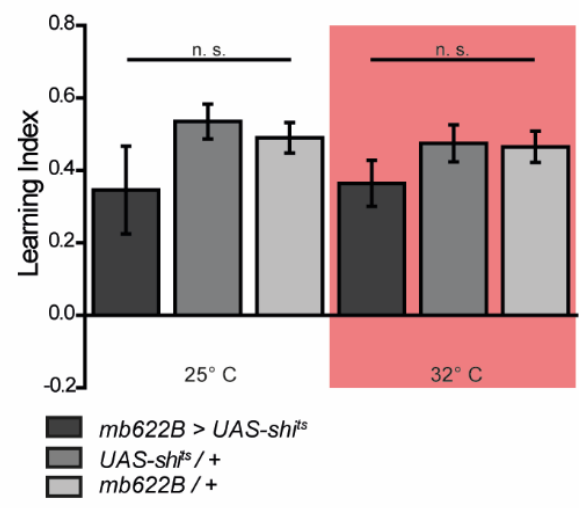

D)

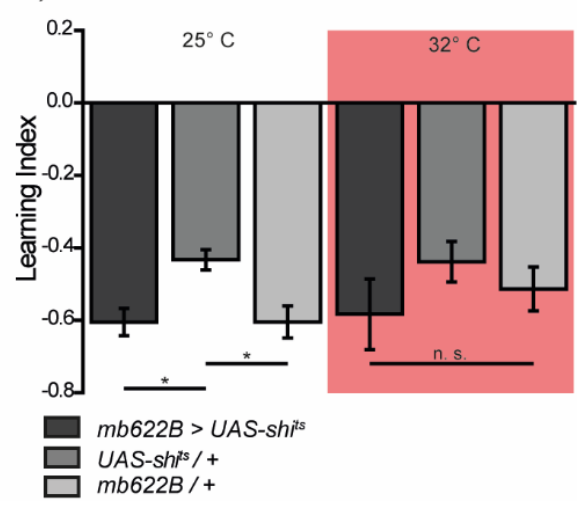

Figure 7.3.: MB-CP1 neurons are not required for short-term memory. Neither odour-sugar (A and B) or odour-electric shock ( $C$ and $D$ ) learning are affected by the inhibition of synaptic output from MB-CP1 neurons. Positive learning indices indicate a preference for the paired odour (CS+), negative values indicate preference for nonpaired odour (CS-). White background indicates experiments that were conducted at permissive temperature (at which shibire $^{\text {ts }}$ is not active), red background indicates

restrictive temperature (at which shibire ${ }^{\text {ts }}$ is active and synaptic output is inhibited). Bars and error bars show mean and SEM, respectively. Difference between groups tested with one-way ANOVA with Bonferroni correction. Learning experiments carried out by Haiko Poppinga. 


\subsubsection{MB-CP1 neurons exhibit a narrow and hard-wired odour tuning profile}

A previous study on the olfactory response profiles of Drosophila MBONs demonstrated that the MBCP1 neurons are relatively finely tuned compared to other MBON types (Hige, Aso, Rubin, et al., 2015). In fact, Hige and colleagues demonstrated, using in vivo calcium imaging, that whereas most MBON types displayed broad responsivity to a panel of odorants, the MB-CP1 neurons showed responses only to the smells of yeast and vinegar (Hige, Aso, Rubin, et al., 2015). These odours are - to a fruit fly - generally highly attractive and signify an optimal environment for feeding, mating, and laying eggs (Dweck et al., 2013; Lebreton et al., 2012; Lin et al., 2015; Sachse \& Beshel, 2016).

Our continued interest in the functional role of the MB-CP1 neurons was thus twofold; firstly, such a response profile implies a link between these neurons and behaviours that are vital for survival and therefore biologically interesting; and secondly, the discrepancy between the broad putative olfactory input and narrow response profile raises questions about the presence and mechanisms of potential long-term plasticity of olfactory-MB circuitry.

To address the latter, we expressed a genetically encoded calcium indicator in the MB-CP1 neurons and presented flies with a panel of odorants under the microscope. Like Hige et al. (2015), we used a variety of odour types and recorded, in vivo, the changes in the intensity of the fluorescence emitted by the calcium indicator as a proxy for neuronal activity. We exposed each fly to six different olfactory stimuli: 4-methylcycohexanol ( $\mathrm{MCH})$, 3-octanol (Oct), benzaldehyde (BA), 11-cis-vaccenyl acetate (cVA), apple vinegar, and a vial of homogenised standard fly food medium.

11-cis-vaccenyl acetate is a male-released aggregation pheromone that also plays an important role in modulating courtship and mating behaviours (Brieger \& Butterworth, 1970; Ejima, 2015). Apple vinegar, produced by fermenting fruits on which Drosophila feed and lay eggs, is highly innately attractive to fruit flies (Zhu et al., 2003). We assume that the fly food medium that flies are raised on in laboratory settings would also be attractive in the same context. Conversely, we also included BA as an odour that is innately aversive, particularly at high concentrations as we used here (Devaud, 2003). MCH and Oct are commonly used in olfactory conditioning experiments and at relatively low concentrations, as used here, do not elicit strong behavioural responses.

To investigate whether the tuning of the MB-CP1 neurons is due to a long-term exposure to "typical" fruit fly food odours (i.e., due to rearing on food of low pH and high yeast content), we experimentally added non-food odours to the developmental environment of the flies. We raised flies from the embryonic stage in food vials into which we had placed a small tube containing $\mathrm{MCH}$ or Oct. As a control, we also raised one group of flies under normal food conditions. Flies that were raised under 
each of these conditions were then aged to 3-6 days into adulthood before conducting in vivo calcium imaging experiments.

In the control group, we observed that the largest odour-evoked activity is indeed in response to apple vinegar (Figure 7.4., A and B; Figure 7.5., A and B) as shown by Hige et al. (2015). Calcium peaks of lower magnitude were observed for the other odorants, although $\mathrm{CVA}, \mathrm{MCH}$, and $\mathrm{BA}$ rarely elicited strong responses in these neurons. The flies that were raised with the smell of 3-Octanol throughout development did not show any increase in the MB-CP1 neuron response to this odour (Figure 7.4., C and D; Figure 7.5., C and D). In fact, raising the flies under these conditions did not lead to any statistically significant change in the response amplitude following presentation of any of our panel of odours (Figure 7.4., G-J; Figure 7.5., G-J). Likewise, flies raised with the smell of MCH also did not show an increased $\mathrm{MB}-\mathrm{CP} 1$ neuron response to $\mathrm{MCH}$. Rather, the long-term exposure of flies to $\mathrm{MCH}$ seemed to result in a notable - although not statistically significant - reduction in responses to $\mathrm{MCH}$, $B A$, and CVA when using the MB242A driver line, with inhibition being observed in some cases (Figure 7.4., E and F; Figure 7.5., E and F).

Hige et al. (2015) reported that the MB-CP1 neurons responded strongly to both apple vinegar and a yeast paste mixture. Although we recapitulate the former, we did not observe particularly strong responses to our standard fly food mixture - which contains yeast extract - in any of our experiments (Figures 7.4 and 7.5.). This, combined with our finding that the response profiles of the MB-CP1 neurons were not skewed toward 3-octanol or $\mathrm{MCH}$ in our long-term exposure experiments, supports the rejection of our hypothesis that this tuning is dependent on the smell of the developmental environment of the flies. 
A)

\section{Fly food}

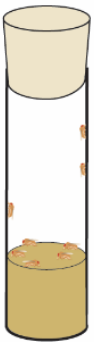

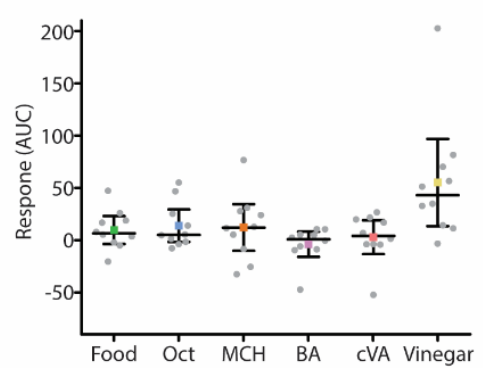

B)
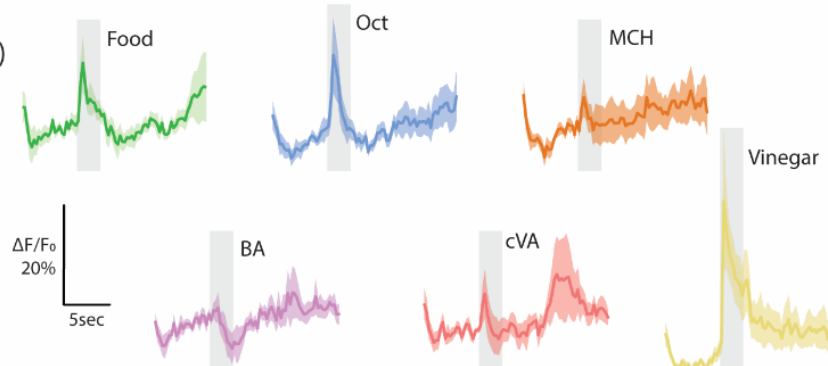

Fly food + Oct

C)

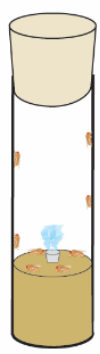

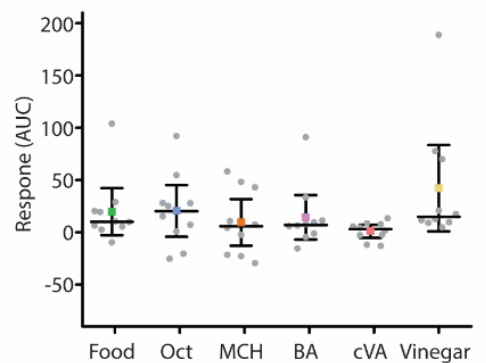

D)
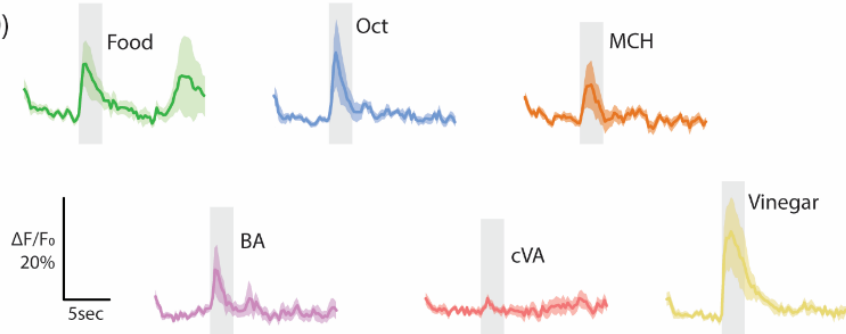

Fly food $+\mathrm{MCH}$

E)

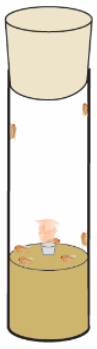

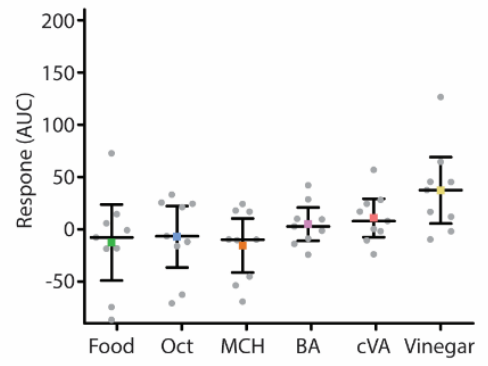

F)
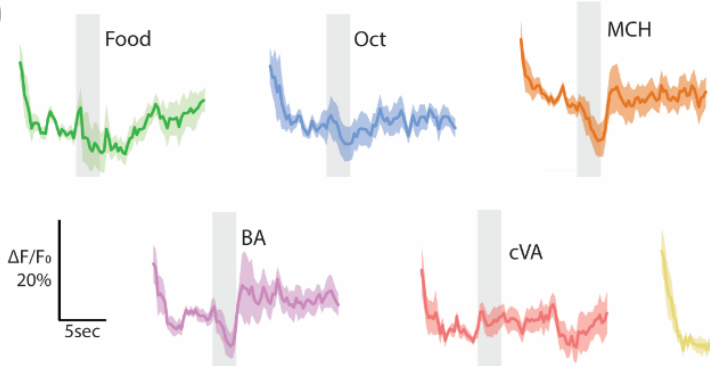

Vinegar
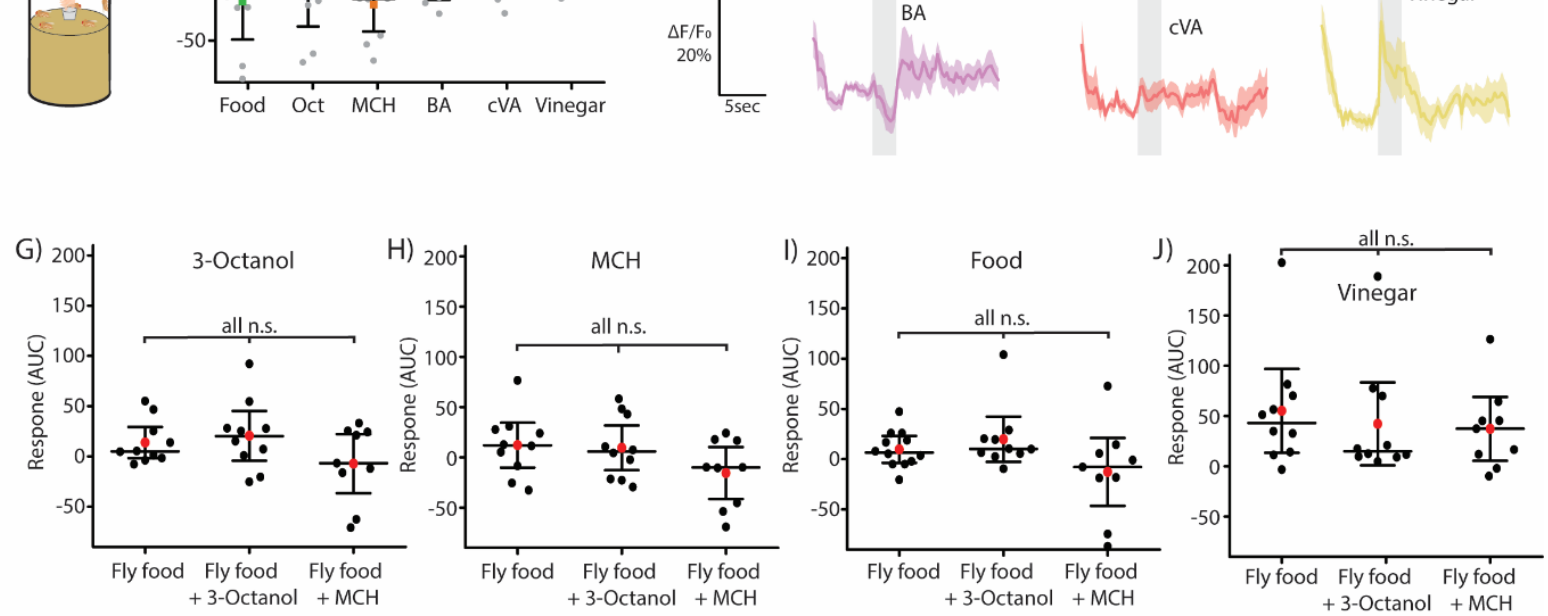

Figure 7.4.: MB-CP1 neuron odour tuning after rearing under variable olfactory conditions using broader MB242A driver line. (A) A control group, raised under normal laboratory conditions, not exposed to an additional odorant through development (left schematic). Responses to the six experimental odours are quantified by the area under the curve (AUC) of the $\Delta F / F_{0}$ traces generated for each odour. Mean $\Delta F / F_{0}$ traces can be seen in (B), with the shaded area indicating the standard error of the mean. Grey bars indicate odour presentation. (C) One experimental group was raised under standard laboratory conditions, with the addition of a small vial of Oct added to the food throughout development, to expose the flies to this odour as a fixture of the developmental environment. (C) and (D) plots as in (A) and (B), respectively. (E) A second experimental group was raised on standard food with the addition of a small vial of $M C H$. (E) and (F) plots as in (A) and (B). (G)- 
(J) To test whether long-term, developmental exposure to an odour conferred a later MB-CP1 neuron response to that odour, we compare responses to experimental odours Oct $(G)$ and $M C H(H)$ and food odours (I) and (J). $n$ values for each group: fly food only group, $n=10$ flies; food + Oct, $n=10$ flies; food $+\mathrm{MCH}, \mathrm{n}=9$ flies. Comparisons between groups conducted with Kruskal-Wallis ANOVA and found no significant differences.

Fly food

A)

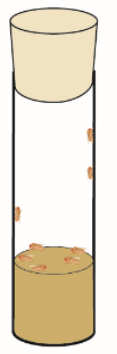

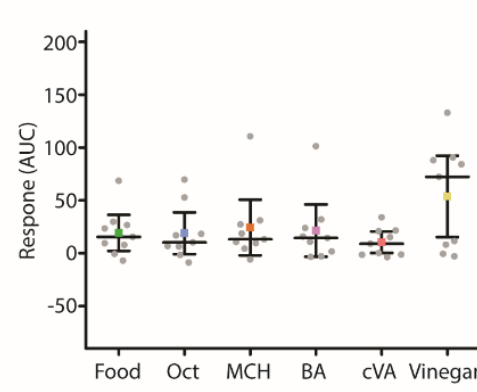

B)
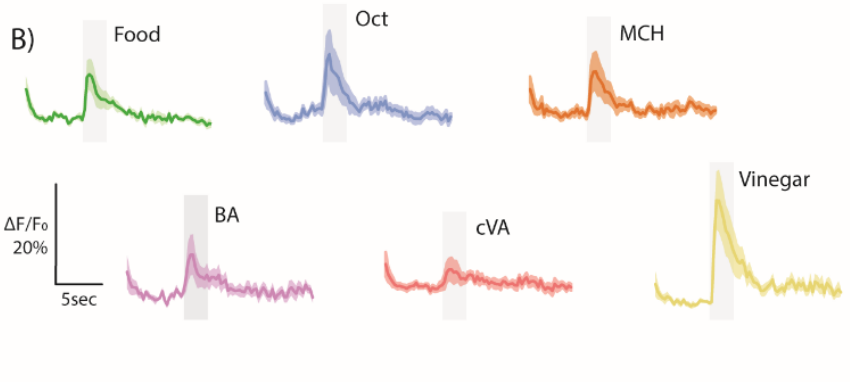

Fly food + Oct

C)

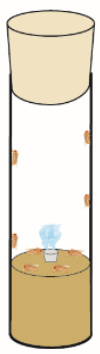

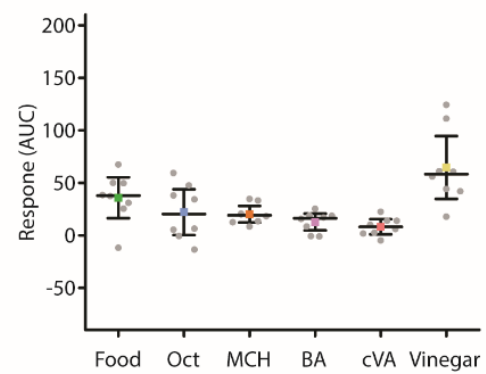

D)

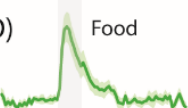

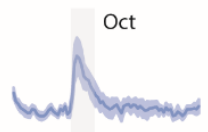

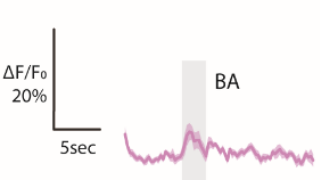

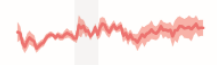
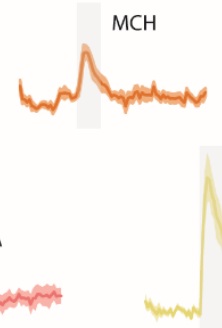

Fly food $+\mathrm{MCH}$

E)

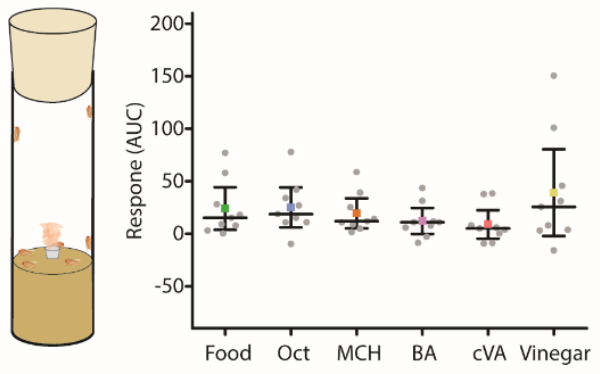

F)
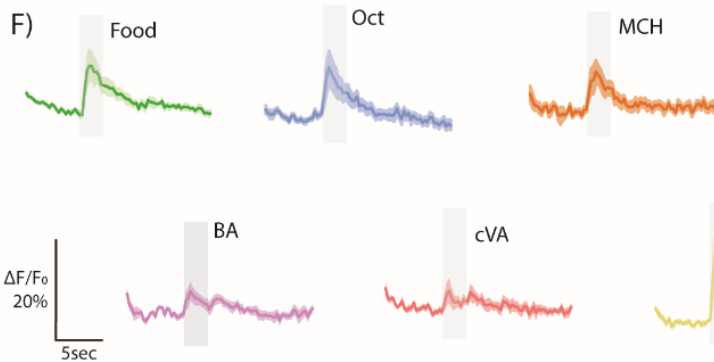
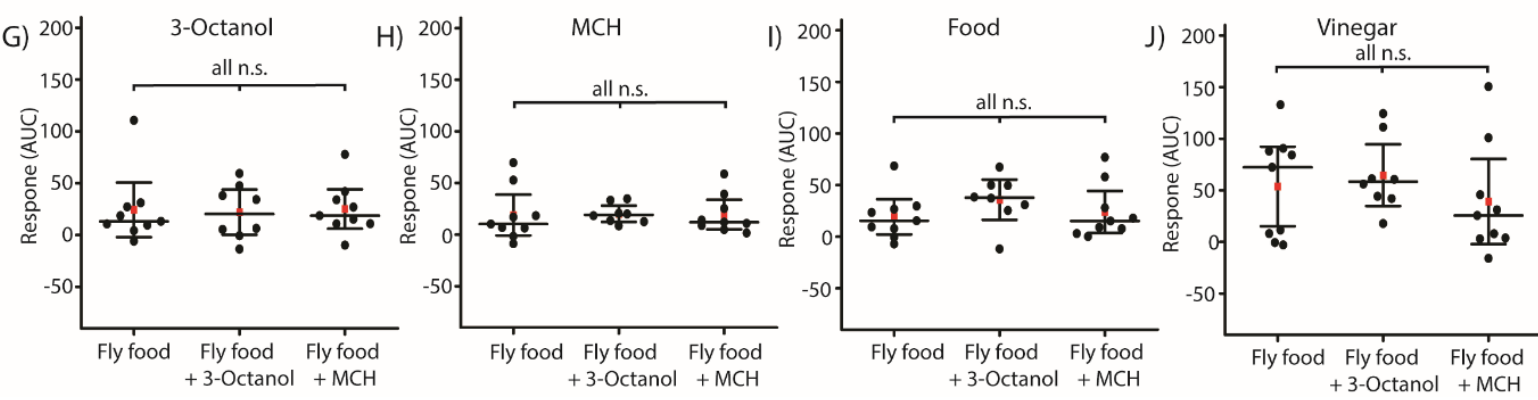

Figure 7.5.: MB-CP1 neuron odour tuning after rearing under variable olfactory conditions using single MBON-targeting driver MB622B. (A) A control group, raised under normal laboratory conditions, not exposed to an additional odorant through development (left schematic). Responses to 
the six experimental odours are quantified by the area under the curve (AUC) of the $\Delta F / F_{0}$ traces generated for each odour. Mean $\Delta F / F_{0}$ traces can be seen in (B), with the shaded area indicating the standard error. Grey bars indicate odour presentation. (C) One experimental group was raised under standard laboratory conditions, with the addition of a small vial of Oct added to the food throughout development, to expose the flies to this odour as a fixture of the developmental environment. (C) and (D) plots as in (A) and (B), respectively. (E) A second experimental group was raised on standard food with the addition of a small vial of $\mathrm{MCH}$. (E) and (F) plots as in (A) and (B). (G)-(J) To test whether longterm, developmental exposure to an odour conferred a later MB-CP1 neuron response, we compare responses to experimental odours Oct $(\mathrm{G})$ and $\mathrm{MCH}(\mathrm{H})$ and food odours $(\mathrm{I})$ and $(\mathrm{J})$. $\mathrm{n}$ values for each group: fly food only group, $n=9$ flies; food + Oct, $n=8$ flies; food $+M C H, n=9$ flies. Comparisons between groups conducted with Kruskal-Wallis ANOVA and found no significant differences.

\subsubsection{The influence of hunger and sex on MB-CP1 neuron odour tuning}

Having concluded that the odour response profile of the MB-CP1 neurons is likely hard-wired, we questioned whether responses to vinegar and yeast odours may instead imply a link to innate odourdriven behaviours. Locating and navigating toward food and potential mates, for example, are two such behaviours that are strongly linked to olfaction (Lebreton et al., 2012; Lin et al., 2015). All previous functional imaging experiments were conducted using satiated female flies - that is, flies that lacked a strong food-seeking drive at the time of experiments.

To address the possibility that the activity of the MB-CP1 neurons is related to the direction of feeding behaviours, we starved female flies for 20-24 hours before functional imaging experiments. At this time point, flies should be in a state of hunger such that food seeking is elevated. We did not observe any significant changes to the response profiles of the MB-CP1 neurons in starved flies compared to a satiated control group (Figure 7.6. and Figure 7.7.). From these data, we infer that the level of activity of the MB-CP1 neurons - to either food or non-food odours - is not directly related to the hunger state of the animal. 
Fly food

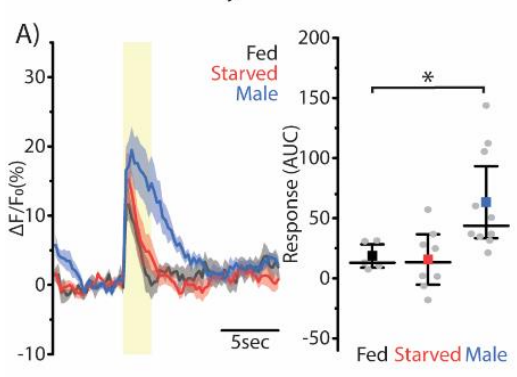

Benzaldehyde

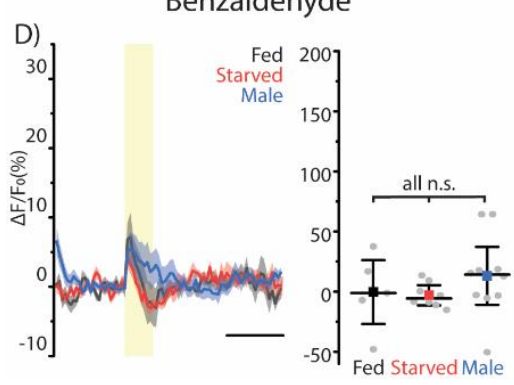

3-Octanol

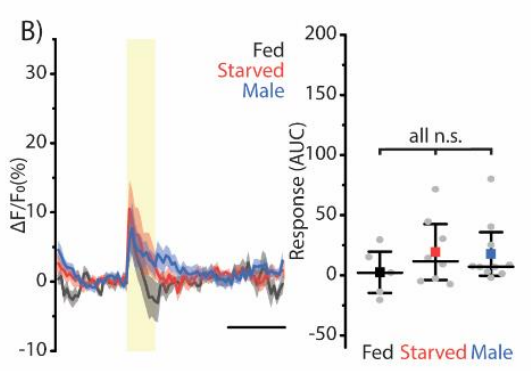

cis-Vaccenyl Acetate

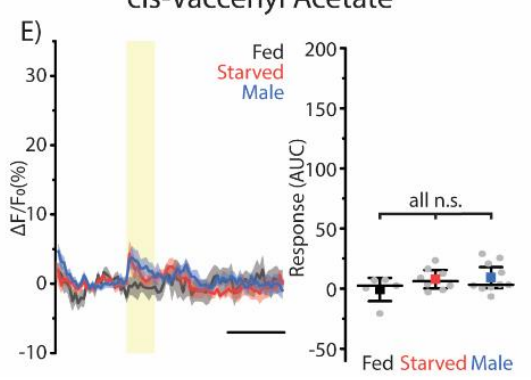

Methylcyclohexanol

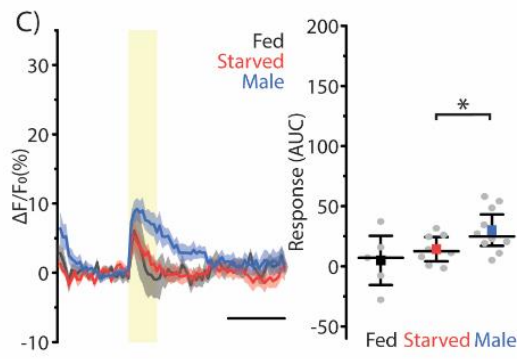

Apple vinegar

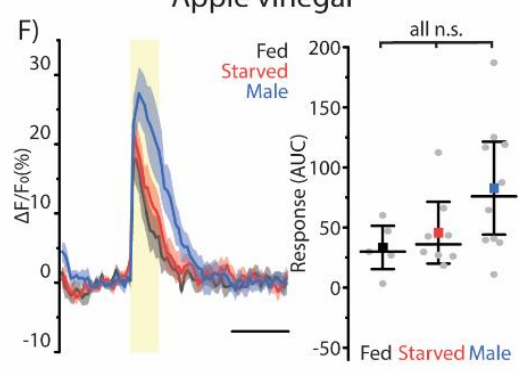

Figure 7.6.: Male flies show stronger responses to food odours in MB-CP1 neurons (under MB242A driver expression). Responses to the six experimental odours are compared between fed female (black), starved female (red), and fed male (blue) flies. Lines indicate mean $\Delta F / F_{0}$ values over time and shaded areas indicate standard error of mean. Scatter plots show median responses quantified as area under curve $(A \cup C)$ of individual $\triangle F / F_{0}$ traces. Upper and lower error bars indicate $95 \%$ confidence intervals. Differences between groups were tested using Kruskal-Wallis ANOVA with Dunn's post-hoc test.

Fly food

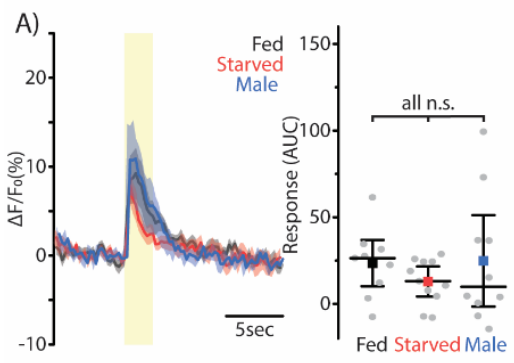

Benzaldehyde

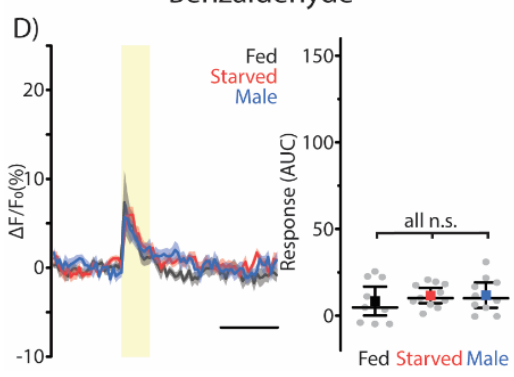

3-Octanol

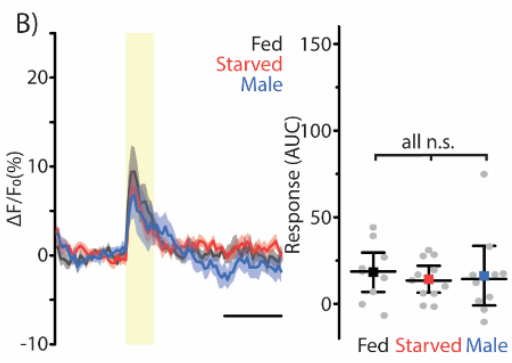

cis-Vaccenyl Acetate

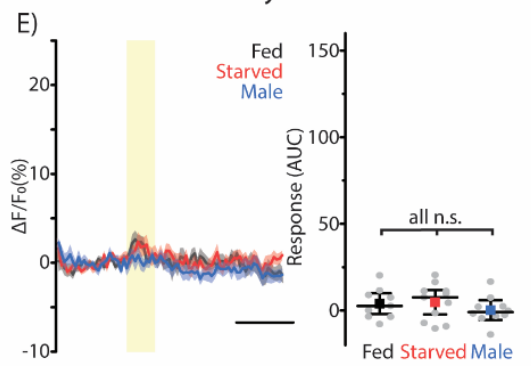

Methylcyclohexanol

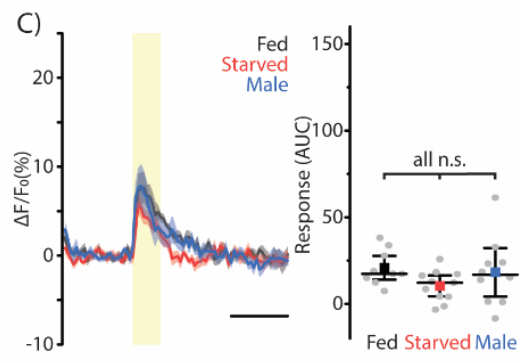

Apple vinegar

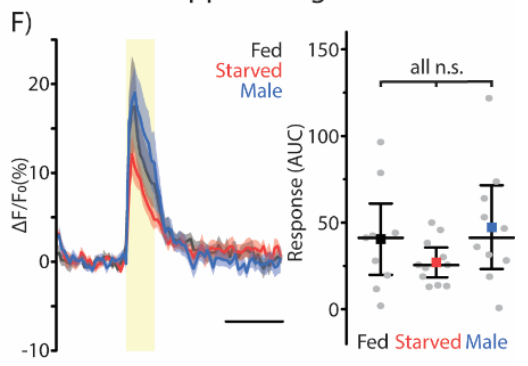

Figure 7.7. Sex and hunger-state do not influence single MB-CP1 neuron odour tuning (under MB622B driver expression). Responses to the six experimental odours are compared between fed female (black), starved female (red), and fed male (blue) flies. Lines indicate mean $\Delta F / F_{0}$ values over 
time and shaded areas indicate standard error of mean. Scatter plots show median responses quantified as area under curve (AUC) of individual $\Delta F / F_{0}$ traces. Upper and lower error bars indicate 95\% confidence intervals. Differences between groups were tested using Kruskal-Wallis ANOVA with Dunn's post-hoc test.

In male fruit flies, the smell of food-related odours has an additional level of importance because food is often associated with the presence of potential mates. Therefore, we next measured the MB-CP1 neurons of (satiated) male flies to investigate any possible sexual dimorphism in the odour-evoked activity in these neurons. Using the MB242A driver line, we observed a significantly higher response to the smell of our standard fly medium in satiated males when compared to satiated females (Figure 7.6., A). We also observed an elevation in the response to $\mathrm{MCH}$ in satiated male flies compared to starved female flies with this driver (Figure 7.6., C), although an additional experiment in which one monitored starved male flies would be needed to elucidate if this is a hunger state-dependent sexual dimorphism, or a difference specifically between starved female and fed male flies. Although not statistically significant, male flies also showed an increase in response to apple vinegar (Figure 7.6., F). None of these observed differences were seen when visualising single MB-CP1 neurons perhemisphere with the MB622B driver line (Figure 7.7.).

From these data, we conclude that the odour response profile of the MB-CP1 neurons is not dependent on the hunger/satiety state of the animals, but that the additional MB-CP1 neuron per hemisphere that is targeted by the MB242A driver appears to display some sexually dimorphic characteristics - the possible behavioural implications of which bear further investigation. 


\subsection{Discussion}

This work is primarily focussed on investigating how experience shapes neural circuits and their functional properties. While in previous chapters we discussed this in the context of olfactory learning, here we were interested in the factors that influence naïve odour coding, using a specific mushroom body output neuron (MBON) selected for its distinctive properties. As the downstream targets of hundreds of odour-coding Kenyon cells (KCs), most MBONs display a broad odour tuning profile whereby almost any given odour results in their activation. This is not the case for the pair of MBONs that innervate the calyx of the mushroom body - what we have called here the MB-CP1 neurons.

A previous functional imaging study showed that one of the MB-CP1 neurons responds very specifically to the smells of vinegar and yeast (Hige, Aso, Rubin, et al., 2015), despite very dense and broad dendritic inputs to these neurons in the MB calyx (which receives strong and direct input from the olfactory system). We first hypothesised that this may be a characteristic that arises through the developmental life of the fly, which takes place in a food environment that is acidic and rich in yeast aroma, due to the low $\mathrm{pH}$ and addition of yeast extract to the fly medium used in most Drosophila research labs, and may therefore lead to a specific strengthening of the synaptic connections between olfactory inputs conveying those smells of the developmental environment and the MB-CP1 neurons. Our data suggest, however, that this is not the case: the addition of non-food odours to the developmental environment of the flies did not result in the addition of that odour to the response profile of the MB-CP1 neurons in the fully developed fly (Figures 7.4. and 7.5.). This shifted the focus of the study to other factors that can influence responsiveness to olfactory stimuli - hunger state and sexual dimorphism. Therein, we observed a significantly stronger MB-CP1 neuron response to food in male flies than females, and no differences in responses when flies were starved for the day preceding experiments (Figures 7.6. and 7.7.).

Future work will focus on the possible behavioural implications of the activity of these neurons. A clear candidate, based on data obtained here, is courtship behaviour - a sexually dimorphic and highly olfaction-driven process (e.g., reviewed by Ziegler et al., 2013). Another possibility is a role in feeding behaviours, whereby activity in the MB-CP1 neuron is involved in the relay of information about possible feeding sites. By exploiting the array of genetic tools available in Drosophila, the necessity and/or sufficiency of the activity of these neurons in execution of these, or myriad other, behaviours can be tested by observing behaviour of flies in which these neurons are artificially activated or silenced. 


\section{General discussion}

One of the most prominent and longstanding endeavours of biologists is understanding how and why animals behave as they do. As the primary means by which an individual can interact with its environment, tightly controlled behavioural expression has direct consequences for their chance of survival. Indeed, pursuant to the theory of sexual selection and heredity of survival-promoting traits, over the last half-century Drosophila melanogaster as a model system has been used to identify many genes that control vital behaviours that support survival. Rapid technological progress throughout this period has cemented Drosophila as an invaluable model for the investigation of diverse behaviours and the molecular, cellular, and neural network mechanisms underlying their control.

The work presented here has aimed to build on our knowledge of the neural principles that govern olfactory processing, in the context of both naïve and learned stimuli. To this end, two novel technical approaches are described (in sections 3 and 5), as well as the implementation of neural activity monitoring in brain circuits associated with odour processing and memory formation (sections 4, 6, and 7).

\subsection{Circuit dissection at subcellular resolution}

One of the key themes addressed here is the dissection of the neural circuits in the fly brain that are involved in the formation of associative memories. Broadly speaking, this process has been localised to the mushroom body $(\mathrm{MB})$ - a finding that has been iterated many times in the last few decades using techniques from selective MB ablation (Belle \& Heisenberg, 1994) to use of genetically encoded inhibitors of neurotransmission (McGuire et al., 2001). However, such techniques, beyond assigning coarse locality, tell us little about the structural and mechanistic properties of a memory trace or "engram". The latter term was first coined by Semon as a descriptor of the physical manifestation of a memory, considered to exist in the form of distinct changes in the brain (either transient or longlasting) that underly memory formation (Semon, 1921). However, a comprehensive examination of this principle has been limited by the lack of experimental tools with the spatial resolution needed to precisely locate these neural changes.

A key development in this vein has therefore been the advances in tools for the monitoring of neural activity at single-cell resolution. Visualisation of activity using genetically encoded calcium indicators (GECls) is one such advance, with rapid developments including improved iterations of GCaMP occurring consistently over the last 20 years (Akerboom et al., 2012; Chen et al., 2013; Dana et al., 2019; Nakai et al., 2001; Tian et al., 2009). By expressing one of these GECls in neurons of interest 
and observing neural activity while exposing animals to a training paradigm that induces learning, it is therefore possible to observe the activity changes in the brain that coincide with the process of learning in vivo and in real time. Such techniques were described here in sections 3 and 5.

With these methods, we selectively targeted the MB intrinsic Kenyon cells (KCs; in section 5) or the $\mathrm{MB}$ output neurons (MBONs; in section 3). Considering the key role of the KCs in associative learning, relatively few studies have examined their activity during this process, and none at the spatial resolution likely necessary to observe small, localised changes in activity. Generally, two main optical imaging approaches have been employed: either measurement of calcium transients at the level of the KC somata (e.g., Dylla et al., 2017; Lüdke et al., 2018)- which allows for characterisation of single $\mathrm{KCs}$, but likely excludes the influence of the local circuitry of the $\mathrm{KC}$ axons that is known to be vital for associations to take place (Heisenberg, 2003); or measurement at the axonal level (e.g., Akalal et al., 2010; Wang et al., 2008), where short-term plastic changes are more likely to be found but single cell resolution is lost due to the dense packing of hundreds of axonal arbours (Aso et al., 2014a; Tanaka et al., 2008). These techniques have utilised the genetic expression systems available in Drosophila (see section 1.2.) to selectively express GECls in the $2000 \mathrm{KCs}$ of the MB (Aso et al., 2009; Aso et al., 2014a; Li et al., 2020). By using an adapted version of the classic Gal4/UAS system, we demonstrated a method whereby this expression is limited to single $\gamma$-type KCs per fly using the MARCM method (see section 5, and Hancock et al., 2020). In doing so, it is possible to visualise naïve and learning-modulated odour-evoked calcium responses in single synaptic boutons. These synaptic boutons are distinct structures found along the KC axons that are enriched with presynaptic proteins (e.g., active zone protein Bruchpilot (BRP); see section 6 Figure 2) making them likely sites of KC neurotransmitter release, and therefore a favourable candidate structure when looking for learning mediated plasticity in this circuit.

This approach furthermore allowed for functional plasticity to be analysed with consideration of differential neuromodulation along the $\mathrm{KC}$ axon. The $\mathrm{MB} \gamma$-lobe is compartmentalised such that spatially distinct, non-overlapping inputs from dopaminergic neurons (DANs) that convey the presence of reinforcing stimuli during training (Aso et al., 2012; Aso et al., 2014a; Cohn et al., 2015) are exerted across the lobe. Crucially, each KC of the $\mathrm{y}$-lobe projects its axons through the length of the lobe and thus passes through each of these compartments. This provides a substrate by which different synaptic boutons along the same cell could theoretically be subjected to differential modulation through learning - a phenomenon that can be visualised using the technique described in section 5 . By considering each of these boutons as an individual unit and categorising them dependent on the compartment in which they reside, it is also possible to investigate odour coding in the context of the combined activity of boutons rather than cells. Thus, congruence between boutons in the 
context of their anatomical position is found to be a novel parameter by which odour representations can be analysed.

As the primary downstream targets of the MB KCs, the MBONs are also of interest in the dissection of associative learning circuits. In section 3, we described the use of a form of GCaMP that localises specifically to post-synaptic sites via fusion to the post-synaptic matrix protein Homer (developed by Pech et al., 2015). While several studies have analysed learning-induced modulation of odour-evoked activity in MBONs using in vivo calcium imaging techniques (Berry et al., 2018; Owald et al., 2015b; Perisse et al., 2016), we demonstrate that the use of homer-GCaMP presents a more precise tool in this endeavour. Given the complex feedback/feedforward structure of the MB and MBON network (Li et al., 2020a; Takemura et al., 2017), calcium transients measured at the MBON axon or cell body layer are likely highly influenced by inputs of multiple other cell types that occur downstream (such as other MBONs and DANs), and not only the KCs. Although one cannot define from which population of neurons an input is derived with this method, spatially restricting the localisation of the calcium sensor to sites of majority $\mathrm{KC}$ connectivity provides a more precise picture of the information received from the MB.

These two methodological approaches therefore demonstrate adaptations to the commonly used method of in vivo calcium imaging in Drosophila such that a greater level of spatial precision is achievable in discerning sites of learning-induced plasticity. These methods were implemented in sections 4 and 6 of this work, the ramifications of which are discussed in the next sections.

\subsection{Mushroom body compartmentalisation: implications for associative learning}

\subsubsection{From odour coding to valence coding}

Early anatomical studies of the MB and its associated circuitry identified the presence of a number of so-called mushroom body extrinsic neurons, defined broadly as neurons that form either efferent or afferent connections between the MB and other brain regions (Ito et al., 1998; Tanaka et al., 2008). These extrinsic neurons display a tiling innervation of the MB lobes, whereby discrete borders exist between the MB regions innervated by different extrinsic neuron types. The majority of these extrinsic neurons have since been characterised as either dopaminergic neurons (DANs) or mushroom body output neurons (MBONs) and have been anatomically mapped in detail using specific Gal4 and splitGal4 driver lines (Aso et al., 2014a). Aso et al. (2014b) also showed that, as well as being anatomically distinct, the MBONs possess distinct functional properties dependent on the compartments that they 
innervate. This included the finding that the optogenetic activation of some MBON types resulted in a promotion of approach behaviour, while others promoted avoidance behaviour (Aso et al., 2014b). This has thus led to the development of a MBON valence-coding model, whereby the relative activation of approach- and avoidance-promoting MBONs elicited by a stimulus is informative of its valence. Indeed, this principle supports the classic model for the mechanism underlying associative learning in the $\mathrm{MB}$, whereby plastic changes at the synapses between $\mathrm{KCs}$ and $\mathrm{MBONs}$ represent the site at which associative olfactory memories are localised (Heisenberg, 2003) - with the shifting of MBON activity patterns representing the early neural basis of learned approach or avoidance behaviour.

In terms of odour processing, this also implies that relative odour drive from $\mathrm{KC}$ to $\mathrm{MBON}$ is variable dependent on MB compartment - such that the activation of the MBON network reflects the odour valence. Presumably then, when a fly encounters an odour to which it has not previously been exposed (and thus holds no strong valence), the odour-evoked activity observed across the approach- and avoidance-promoting MBONs would be balanced to reflect this. In Figure 4.2., we tested exactly this hypothesis by expressing the postsynaptically localised GECI homer-GCaMP in each of the $y$-lobe innervating $\mathrm{MBONs}$ and monitoring their activity during odour presentation. Approach-promoting MBONs innervating the $\gamma 1$ and $\gamma 2$ compartments and the avoidance-promoting MBON of the $\gamma 4$ compartment all showed very similar naïve odour response amplitudes and dynamics (Figure 4.2., A). Notably, responses of MBON- $\gamma 3$ appear more robust than the others, while MBON- $\gamma 5$ responses were very rare (as also previously reported by Owald et al. (2015)). We also observed these characteristics when measuring at the level of the KC presynapse (section 6, Figure 4), where the median odour response amplitudes of boutons were equal between all compartments but the congruence of responses within compartments was variable such that $\gamma 3$ boutons acted most cohesively and those of $\gamma 5$ least cohesively - although all compartments showed greater internal than cross-compartment congruence. Therefore, the degree of compartment-wise cohesion of odour responses at the KC bouton level appears to be a characteristic that influences the level of input drive to the MBONs.

\subsubsection{Locating the memory trace}

Classical olfactory conditioning leads to the learned association between an odour stimulus (CS+) and an appetitive or aversive stimulus (US; Pavlov, 1927). In turn, this association is expressed as a learned attraction to or avoidance of the conditioned odour (Tempel et al., 1983; Tully \& Quinn, 1985). The requirement for MB function for these associations to take place (Heisenberg et al., 1985; McGuire et al., 2001; Zars et al., 2000a) and the putative valence-coding role of the MBONs that lie immediately 
downstream from the MB (Aso et al., 2014b) have made the MBONs a target of many recent studies of learning and memory formation. Indeed, the classical model for the neural correlates of associative learning - i.e., the hypothesised dopamine-mediated modulation of KC-to-MBON synapses (Heisenberg, 2003) - has been expanded upon to account for the existence of distinct MBON types that encode opposite valences. Functional imaging experiments, for example, have shown that responses to aversively paired odours are reduced in approach-promoting MBONs and potentiated in avoidance-promoting MBONs (Berry et al., 2018; Owald et al., 2015; Perisse et al., 2016), supporting the theory that the activity of specific MBON types correlate with approach or avoidance behaviour and that this activity is modifiable through learning. Indeed, Hige et al. (2015) showed that this modification of MBON activity is directly linked to the activity of DANs by substituting the electric shock (US) with optogenetic activation of electric shock-responsive DANs and observing the same effect (although using electrophysiological recording, rather than optical techniques).

In section 4, we sought to test the theory that the net activity across $y$-lobe MBONs is modified through learning. Where other studies generally monitored single MBONs or pairs of MBONs to find sites at which learning-mediated plasticity occurs, we instead monitored postsynaptic odour-evoked calcium dynamics in each of the $\gamma$-lobe innervating MBONs before and after an associative conditioning procedure. What we observed, however, provides evidence that modulation of odour-evoked input to the MBONs does not occur across the entire $y$-lobe but is rather localised to a single compartment. After conditioning, MBON- $\gamma 1$ (identified as an approach-promoting MBON (Aso et al., 2014b)) showed a robust and specific suppression to its response to an aversively paired odour (Figure 4.3.). This is cohesive with previous studies (Hige, Aso, Modi, et al., 2015; Perisse et al., 2016) and the theory that learning to associate an odour with a punishing electric shock leads to a shift in MBON responses to that odour such that approach behaviour is suppressed upon subsequent exposure.

This theory, however, does not hold true with our findings from the remaining $\gamma$-lobe MBONs. In fact, we did not observe any specific associative effects on the activity of the MBONs that innervate compartments $\gamma 2-\gamma 5$ (Figure 4.4.). In all cases, responses in these neurons either did not significantly change or showed depression. MBON- $\gamma 2$ and $\mathrm{MBON}-\gamma 3$ showed strong depression of odour-evoked activity after both the paired (aversive conditioning) protocol and the CS-only control protocol (in which flies only received odour presentation without US), implying that the prolonged odour presentation used in both of these protocols may lead to an adaptation effect in these MBONs. As such, it is particularly notable that MBON- $\gamma 3$ odour responses after the US-Only control protocol (in which flies receive only the electric shock punishment without odour presentation) are not significantly changed from the pretraining response, and MBON- $\gamma 2$ depression is much less severe after the paired protocol than the odour only. This latter observation may also indicate a potential 
modulatory role of the electric shock such that its presentation, with or without accompanying odour, also influences subsequent odour-evoked activity of these MBONs perhaps as a form of sensitisation. Like MBON- $\gamma 2$ and MBON- -2 , odour-evoked activity in MBON- -4 showed a similar trend wherein the conditioning and the control procedures led to a depression of post-training responses. Unlike the other MBONs, depression of $\mathrm{MBON}-\mathrm{\gamma} 4$ was similar between all training conditions and odours, although perhaps slightly more robust in flies that received the associative conditioning procedure. All of these effects observed in MBON- $\gamma 2, M B O N-\gamma 3$, and MBON- $\gamma 4$ are, however, distinctly nonassociative. That is, none of these neurons show specific modulation in response to the aversively paired odour (CS+), but rather display generalised effects that occur independent of training status and that are likely due to adaptation after prolonged or repeated odour presentation.

Overall, we conclude from these data that, at the level of the MBON postsynapse, there is no observable memory trace outside of the $\gamma 1$ compartment. The circuitry of the $\gamma 1$ compartment has been noted by other studies as having distinct qualities compared to other compartments. For example, the activity of the dopaminergic input neuron to the $\gamma 1$ compartment (PPL1- $\gamma 1$ pedc) has been found to be both necessary and sufficient for the induction of short-term aversive olfactory learning, strongly responsive to and, via controlled activation during conditioning, capable of replacing the electric shock US in induced aversive associative learning (Aso et al., 2010; Aso et al., 2014b; Hige, Aso, Modi, et al., 2015). MBON- $\gamma 1$ is also one of only three MBONs that has axonal terminals within the $M B$, implying a possible feedback role of this neuron. Indeed, light microscopy and connectomic studies have identified that MBON- $\gamma 1$ has the potential to form presynaptic connections with five to six additional MBON types, including its contralateral counterpart (Aso et al., 2014a, 2014b; Li et al., 2020) and the functional evidence of $M B O N-\gamma 1$ feedback has been demonstrated in MBONs of the $\beta^{\prime} 2$ region (Felsenberg et al., 2018; Perisse et al., 2016).

Critically, we lack the data for the presynaptic KCs in the $\gamma 1$ compartment due to the technical infeasibility of measuring this region together with $\gamma 2-\gamma 5-$ as the $\gamma 1$ compartment lies deeper in the brain and would require specific experimental targeting. Having now additionally identified the MBON- $\gamma 1$ postsynapse as a site of learning-induced plasticity, it would of course be beneficial in the future to investigate the presynaptic KC boutons in this region to elucidate potential presynaptic mechanisms that underly learning-induced changes in odour drive to the MBON.

At the level of the KC presynapse in compartments $\gamma 2$ to $\gamma 5$, we observed that there is a significant reduction in compartment-wise cohesion among synaptic boutons as a result of associative conditioning (section 6, Figure 5). This occurred only in the case of the conditioned odour (CS+), with CS- (which is presented without electric shock) and control odours eliciting response patterns that 
were not significantly different to the naïve, pretraining responses. Surprisingly, however, this learning-induced decorrelation was seen throughout the $y$-lobe with only small differences between compartments, but no indication of differential modulation dependent on the valence encoded by the downstream MBON. Currently, it is not known how KC bouton decorrelation is decoded downstream, but the differentiation of the learned CS+ and the CS- or control odours is not observed at the MBON postsynapse level across the $\gamma 2$ to $\gamma 5$ compartments.

However, robust bidirectional changes in MBON activity have been reported previously, with approach-promoting MBON responses to an aversively trained odour being reduced while, presumably in parallel, avoidance-promoting MBON responses are increased for this odour (Owald et al., 2015). There are a couple of fundamental experimental differences between this study and ours that may account for our different findings. First, we used a procedure in which flies were conditioned while fixed in place under the microscope and thus have within individual comparisons of odour responses before and after conditioning, whereas others typically condition flies in a classical behavioural setup and then transfer flies to a microscopic setup to monitor post-conditioning odour responses. We have observed that the MBONs show particularly high variability in odour responses across individuals, even before training, making the more controlled use of within individual comparisons favourable over population level comparisons in which these naïve differences are not accounted for. Second, we have used a much more precisely localised postsynapse-targeted GECI (homer-GCaMP), rather than the typical cytosolically localised sensors (e.g., GCaMP6), meaning we quantify a very different signal.

Likewise, the experimental approach to monitoring learning-induced modulation of KC activity we describe here is thus far novel in the field and as such, previous studies have observed different findings. For example, one study reported finding no learning-induced changes in odour responses across compartments of the $y$-lobe after aversive conditioning when recording across the whole population of YKCs (Louis et al., 2018). That we find specific learning-induced changes in odour representations when monitoring at the individual bouton level that is not detectable when imaging across large populations of neurons supports the use of more precise activity monitoring techniques, and also the challenging of the idea of gross activity output as the only parameter that could be instructive of downstream signalling. This principle is also true at the level of the MBONs, where we find that it may be advantageous to observe subcellularly localised activity in order to more precisely dissect the sites of plasticity in the MB circuit. In the future it would also be valuable to develop imaging protocols with which greater spatial resolution can be achieved such that the MBON postsynapse may also be monitored not as a combination of all postsynaptic signal within a compartment, but within limited regions of odour evoked activity. 


\subsection{Investigation of factors influencing naïve odour tuning}

While the olfactory system and MB circuitry provides a robust model for the investigation of learninginduced synaptic plasticity, it is also interesting to decipher how animals respond to more naturalistic stimuli of innate ethological value. From the antennal lobe, olfactory projection neurons relay odour information to two major higher brain regions - the MB and the lateral horn (LH) - for processing. Broadly speaking, the MB has typically been considered to be specialised in processing of learned stimuli while the LH is primarily involved in innate stimulus processing (Belle \& Heisenberg, 1994; Jefferis et al., 2007). However, this strict delineation between these functions likely does not do the complexity of these systems justice. MBONs that receive input exclusively from the $M B$, for example, have been shown to be required for the display of innate odour avoidance (Lewis et al., 2015; Owald et al., 2015; Tsao et al., 2018) and innate behaviours can be suppressed through learning (Baggett et al., 2018). This implies a degree of plasticity in the circuits controlling these behaviours. On the other hand, some circuits in the brain are hardwired and cannot so easily be modified - for example, in the case of sexual dimorphism.

Although further investigation is still pending, we theorise in section 7 that we have identified a case of this sex-specific hardwiring. There, we began our examination of a MBON type that, unlike the $\gamma$ lobe innervating MBONs studied in section 4, show dense innervation of the calycal region of the MB. These MBONs (MB-CP1 neurons) were also distinct in their odour response profile, showing a very narrow odour tuning consisting of robust responses only to fly food-related odours (reported by Hige, Aso, Rubin, et al., 2015). We first ruled out that this response profile is due to the rearing conditions of the flies (in vials of fly food) by adding non-food odours to the developmental environment of the flies, and observing no recruitment of those odours to the response profile of MB-CP1 neurons (Figures 7.4. and 7.5.). An additional experiment in which flies are deprived of olfactory stimuli during development would also be beneficial in confirming that MB-CP1 odour tuning is not determined by olfactory input. This could be carried out by either experimentally limiting the olfactory environment using a synthetic food source that does not have a strong odour (for example a so-called synthetic, "holidic" medium as developed by Piper et al. (2014)), or by using anosmic flies lacking expression of functional olfactory receptors (e.g., by conducting experiments in flies carrying a null mutation in the odorant receptor coreceptor (ORCO)-encoding gene, Or83b (Larsson et al., 2004)).

Odour tuning can also be influenced by internal state. Hungry flies, for example, exhibit more robust responses to food-related odours at the olfactory projection neuron level (Root et al., 2011) and also at the MBON level, measured at the MB lobes (Tsao et al., 2018). Given that the MB-CP1 neurons showed strong responses to food odours, we questioned whether this activity may be linked to food 
search or feeding behaviour and thus be hunger-state dependent. If this were the case, one may presume that under starvation conditions, responses to food stimuli would be facilitated in these neurons. However, we found no difference in MB-CP1 odour responses in flies that were satiated and those that had been starved for 20-24 hours before experiments (Figures 7.6 and 7.7.).

Olfactory detection of a food source is not only salient as an indicator of a potential feeding site, but also of preferential sites for females to lay eggs (Dweck et al., 2013; Zhu et al., 2003) or males to find mates (Lebreton et al., 2012). Given the sexually dimorphic nature of these candidate behaviours, we next checked to see if odour tuning of the MB-CP1 neurons was influenced by sex. Indeed, we observed significantly higher responses to the smell of food and increased responses to apple vinegar (though not statistically significantly) in male flies compared to female flies (Figures 7.6. and 7.7.).

Follow-up experiments will aim to elucidate if and how the activity of the MB-CP1 neurons influences behaviour. Considering the difference observed between males and females in the olfactory responses of these neurons, courtship behaviour is a clear candidate for further investigation. This is a well characterised and multisensory behaviour (including a strong role for olfaction), predominantly carried out by male flies, that can be easily observed under laboratory conditions and combined with methods of neural activation or silencing (e.g., optogenetics, see section 1.2.) to elucidate underlying circuitry (Bastock \& Manning, 1955; Yamamoto \& Koganezawa, 2013). Many neurons that modulate the expression of sexually dimorphic behaviours also do so via expression of sex-specific isoforms of the transcription factor encoding genes fruitless and doublesex that are differentially expressed in male and female flies (Ito et al., 1996; Rideout et al., 2010; Ryner et al., 1996). This may also provide an avenue for future investigation, by probing for colocalization of these isoforms linked to sexually dimorphic behaviours and the MB-CP1 neurons. 


\section{Summary}

The relay of information from external sensory organs to brain centres that ultimately control behavioural output dictates how an animal interacts with its environment. This process is integral to survival, allowing for the identification of and appropriate response to both beneficial and harmful stimuli. Where some stimuli - such as the smell of food or the sight of a looming predator - have innate value, some stimuli gain significance only through experience. The neural principles underlying this experience-dependent acquisition of stimulus value was the main focus of this work, using olfactory associative conditioning in Drosophila as a model system.

Through this type of conditioning, flies can learn to associate an olfactory stimulus (conditioned stimulus, CS+) with an aversive electric shock (unconditioned stimulus, US), leading to a conditioned avoidance of the CS+. In this thesis, I have presented two studies that investigated two different neural populations involved in this process. First, the description and implementation of an in vivo calcium imaging procedure with which odour-evoked activity across a population of valence coding mushroom body output neurons (MBONs) was investigated. Uniquely, this monitoring was specifically localised to postsynaptic sites using homer-GCaMP. Current models of aversive associative conditioning in Drosophila postulate that the coincidence of odour- and electric shock-mediated inputs in the mushroom body Kenyon cells (KCs) leads to a facilitation or depression of the output from the KCs and the MBONs, such that MBONs encoding positive valence are less active and those encoding negative valence are more active - leading to the expression of the conditioned response based on their net combinatorial activity. This, however, was not reflected in the data shown here. In fact, only one of the five MBONs investigated (MBON- $\gamma 1$ ) showed any learning-induced modulation. We propose that this implies a specialised role of the $\gamma 1$ compartment of the mushroom body in learning, warranting further investigation.

A second study presented here focused on the KCs. Monitoring odour-evoked activity at presynaptic structures (here called synaptic boutons) along single KC axons uncovered some novel insights into the odour coding properties of KCs. First, odour responses are not homogenous along KC axons, with boutons being able to act independently of their neighbours. Additionally, by quantifying the degree to which responses across boutons correlated with one another, it became clear that the functional consequences of compartmentalisation of the mushroom body (by MBONs and dopaminergic neurons) can also be observed in the KCs, with intracompartmental cohesion of bouton responses being greater than intercompartmental. Furthermore, this cohesion of odour-evoked activity is dramatically reduced if the odour has previously been associated with electric shock through aversive classical conditioning. This demonstrates a role for individualised synaptic sites (and, more specifically, 
the degree to which they correlate with one another) in the encoding of learned information, rather than gross activity of whole neurons. Notably, we did not observe any learning-induced change at the level of the MBON postsynapses in these regions of the mushroom body, and so the mechanism by which synchrony/asynchrony of dispersed bouton activity is readout is as yet unclear.

A clear caveat in this study was the absence of data for the $\gamma 1$ compartment, excluded due to technical limitations. Given our finding that only the MBON that receives input from this region undergoes learning-induced modulation, it would be of great interest to replicate the KC study presented here with measurement of the $\gamma 1$ compartment. This provides a promising avenue for further research into the neural principles underlying learning-induced plasticity.

Finally, as part of a separate study, I have introduced early findings from a project investigating the properties of MB-CP1 neurons that we have demonstrated to display a narrow, hardwired odour tuning profile that may imply a role in mediation of innate behaviours. These neurons innervate the calyx of the mushroom body, and, unlike most MBONs, we found that they are not involved in learning and memory processes. The MB-CP1 neurons are responsive only to food-related odour stimuli, in a manner we additionally demonstrate is sexually dimorphic, with male flies showing significantly stronger odour-evoked activity. Continuation of this project will aim to investigate any potential role MB-CP1 neurons play in behavioural control, and the underlying molecular regulation therein. 


\section{References}

Akalal, D. B., Yu, D., \& Davis, R. L. (2010). A late-phase, long-term memory trace forms in the gamma neurons of Drosophila mushroom bodies after olfactory classical conditioning. J Neurosci, 30(49), 16699-16708.

Akerboom, J., Chen, T.-W., Wardill, T. J., Tian, L., Marvin, J. S., Mutlu, S., Calderón, N. C., Esposti, F., Borghuis, B. G., Sun, X. R., Gordus, A., Orger, M. B., Portugues, R., Engert, F., Macklin, J. J., Filosa, A., Aggarwal, A., Kerr, R. A., Takagi, R., ... Looger, L. L. (2012). Optimization of a GCaMP Calcium Indicator for Neural Activity Imaging. J Neurosci, 32(40), 13819-13840.

Al-Anzi, B., Armand, E., Nagamei, P., Olszewski, M., Sapin, V., Waters, C., Zinn, K., Wyman, R. J., \& Benzer, S. (2010). The leucokinin pathway and its neurons regulate meal size in Drosophila. Curr Biol, 20(11), 969-978.

Aso, Y., Grübel, K., Busch, S., Friedrich, A. B., Siwanowicz, I., \& Tanimoto, H. (2009). The mushroom body of adult Drosophila characterized by GAL4 drivers. J Neurogenet, 23(1-2), 156-172.

Aso, Y., Hattori, D., Yu, Y., Johnston, R. M., Iyer, N. A., Ngo, T. T. B., Dionne, H., Abbott, L. F., Axel, R., Tanimoto, H., \& Rubin, G. M. (2014a). The neuronal architecture of the mushroom body provides a logic for associative learning. ELife, 3, e04577.

Aso, Y., Herb, A., Ogueta, M., Siwanowicz, I., Templier, T., Friedrich, A. B., Ito, K., Scholz, H., \& Tanimoto, H. (2012). Three dopamine pathways induce aversive odor memories with different stability. PLoS Genet, 8(7), e1002768-e1002768.

Aso, Y., \& Rubin, G. M. (2016). Dopaminergic neurons write and update memories with cell-typespecific rules. ELife, 5, e16135-e16135.

Aso, Y., Sitaraman, D., Ichinose, T., Kaun, K. R., Vogt, K., Belliart-Guérin, G., Plaçais, P. Y., Robie, A. A., Yamagata, N., Schnaitmann, C., Rowell, W. J., Johnston, R. M., Ngo, T. T. B., Chen, N., Korff, W., Nitabach, M. N., Heberlein, U., Preat, T., Branson, K. M., ... Rubin, G. M. (2014b). 
Mushroom body output neurons encode valence and guide memory-based action selection in Drosophila. ELife, 3(3), e04580.

Aso, Y., Siwanowicz, I., Bräcker, L., Ito, K., Kitamoto, T., \& Tanimoto, H. (2010). Specific dopaminergic neurons for the formation of labile aversive memory. Current Biology, 20(16), 1445-1451.

Baggett, V., Mishra, A., Kehrer, A. L., Robinson, A. O., Shaw, P., \& Zars, T. (2018). Place learning overrides innate behaviors in Drosophila. Learn Mem, 25(3), 122-128.

Bang, S., Hyun, S., Hong, S.-T., Kang, J., Jeong, K., Park, J.-J., Choe, J., \& Chung, J. (2011). Dopamine Signalling in Mushroom Bodies Regulates Temperature-Preference Behaviour in Drosophila. PLOS Genet, 7(3), e1001346.

Barnstedt, O., Owald, D., Felsenberg, J., Brain, R., Moszynski, J. P., Talbot, C. B., Perrat, P. N., \& Waddell, S. (2016). Memory-Relevant Mushroom Body Output Synapses Are Cholinergic. Neuron, 89(6), 1237-1247.

Barrows, W. M. (1907). The reactions of the Pomace fly, Drosophila ampelophila loew, to odorous substances. J Exp Zool, 4(4), 515-537.

Bastock, M., \& Manning, A. (1955). The courtship of Drosophila melanogaster. Behaviour, 8, 85-111.

Bates, A. S., Schlegel, P., Roberts, R. J. V., Drummond, N., Tamimi, I. F. M., Turnbull, R., Zhao, X., Marin, E. C., Popovici, P. D., Dhawan, S., Jamasb, A., Javier, A., Capdevila, L. S., Li, F., Rubin, G. M., Waddell, S., Bock, D. D., Costa, M., \& Jefferis, G. S. X. E. (2020). Complete Connectomic Reconstruction of Olfactory Projection Neurons in the Fly Brain. Curr Biol, 30(16), 31833199.e6.

Belle, J. S. de, \& Heisenberg, M. (1994). Associative odor learning in Drosophila abolished by chemical ablation of mushroom bodies. Science, 263(5147), 692-695.

Berry, J. A., Phan, A., \& Davis, R. L. (2018). Dopamine Neurons Mediate Learning and Forgetting through Bidirectional Modulation of a Memory Trace. Cell Rep, 25(3), 651-662.e5.

Blum, A. L., Li, W., Cressy, M., \& Dubnau, J. (2009). Short-and long-term memory in Drosophila require cAMP signaling in distinct neuron types. Curr Biol, 19(16), 1341-1350. 
Boto, T., Louis, T., Jindachomthong, K., Jalink, K., \& Tomchik, S. M. (2014). Dopaminergic modulation of cAMP drives nonlinear plasticity across the Drosophila mushroom body lobes. Curr Biol, 24(8), 822-831.

Bouzaiane, E., Trannoy, S., Scheunemann, L., Plaçais, P. Y., \& Preat, T. (2015). Two Independent Mushroom Body Output Circuits Retrieve the Six Discrete Components of Drosophila Aversive Memory. Cell Rep, 11(8), 1280-1292.

Brand, A. H., \& Perrimon, N. (1993). Targeted gene expression as a means of altering cell fates and generating dominant phenotypes. Development, 118(2), 401-415.

Brieger, G., \& Butterworth, F. M. (1970). Drosophila melanogaster: Identity of Male Lipid in Reproductive System. Science, 167(3922), 1262-1262.

Burke, C. J., Huetteroth, W., Owald, D., Perisse, E., Krashes, M. J., Das, G., Gohl, D., Silies, M., Certel, S., \& Waddell, S. (2012). Layered reward signalling through octopamine and dopamine in Drosophila. Nature, 492(7429), 433-437.

Byers, D., Davis, R. L., \& Jr, J. A. K. (1981). Defect in cyclic AMP phosphodiesterase due to the dunce mutation of learning in Drosophila melanogaster. Nature, 289(5793), 79-81.

Carew, T. J., Walters, E. T., \& Kandel, E. R. (1981). Associative learning in Aplysia: Cellular correlates supporting a conditioned fear hypothesis. Science, 211(4481), 501-504.

Caron, S. J. C., Ruta, V., Abbott, L. F., \& Axel, R. (2013). Random convergence of olfactory inputs in the Drosophila mushroom body. Nature, 497(7447), 113-117.

Carpenter, F. W. (1905). The Reactions of the Pomace Fly (Drosophila ampelophila Loew) to Light, Gravity, and Mechanical Stimulation. The American Naturalist, 39(459), 157-171.

Charlie, N. K., Thomure, A. M., Schade, M. A., \& Miller, K. G. (2006). The Dunce cAMP Phosphodiesterase PDE-4 Negatively Regulates Gas-Dependent and Gas-Independent cAMP Pools in the Caenorhabditis elegans Synaptic Signaling Network. Genetics, 173(1), 111-130.

Chen, S., Lee, A. Y., Bowens, N. M., Huber, R., \& Kravitz, E. A. (2002). Fighting fruit flies: A model system for the study of aggression. Proc Natl Acad Sci, 99(8), 5664-5668. 
Chen, T.-W., Wardill, T. J., Sun, Y., Pulver, S. R., Renninger, S. L., Baohan, A., Schreiter, E. R., Kerr, R. A., Orger, M. B., Jayaraman, V., Looger, L. L., Svoboda, K., \& Kim, D. S. (2013). Ultrasensitive fluorescent proteins for imaging neuronal activity. Nature, 499(7458), 295-300.

Cognigni, P., Felsenberg, J., \& Waddell, S. (2018). Do the right thing: Neural network mechanisms of memory formation, expression and update in Drosophila. Curr Opin Neurobiol, 49, 51-58.

Cohn, R., Morantte, I., \& Ruta, V. (2015). Coordinated and Compartmentalized Neuromodulation Shapes Sensory Processing in Drosophila. Cell, 163(7), 1742-1755.

Crittenden, J. R., Skoulakis, E. M. C., Han, K.-A., Kalderon, D., \& Davis, R. L. (1998). Tripartite mushroom body architecture revealed by antigenic markers. Learn Mem, 5(1), 38-51.

Dana, H., Sun, Y., Mohar, B., Hulse, B. K., Kerlin, A. M., Hasseman, J. P., Tsegaye, G., Tsang, A., Wong, A., Patel, R., Macklin, J. J., Chen, Y., Konnerth, A., Jayaraman, V., Looger, L. L., Schreiter, E. R., Svoboda, K., \& Kim, D. S. (2019). High-performance calcium sensors for imaging activity in neuronal populations and microcompartments. Nature Methods, 16(7), 649-657.

Dankert, H., Wang, L., Hoopfer, E. D., Anderson, D. J., \& Perona, P. (2009). Automated monitoring and analysis of social behavior in Drosophila. Nat Methods, 6(4), 297-303.

Devaud, J.-M. (2003). Experimental studies of adult Drosophila chemosensory behaviour. Behav Processes, 64(2), 177-196.

Dolan, M.-J., Frechter, S., Bates, A. S., Dan, C., Huoviala, P., Roberts, R. J., Schlegel, P., Dhawan, S., Tabano, R., Dionne, H., Christoforou, C., Close, K., Sutcliffe, B., Giuliani, B., Li, F., Costa, M., Ihrke, G., Meissner, G. W., Bock, D. D., ... Jefferis, G. S. (2019). Neurogenetic dissection of the Drosophila lateral horn reveals major outputs, diverse behavioural functions, and interactions with the mushroom body. ELife, 8, e43079.

Dow, M. A., \& von Schilcher, F. (1975). Aggression and mating success in Drosophila melanogaster. Nature, 254(5500), 511-512.

Drain, P., Folkers, E., \& Quinn, W. G. (1991). CAMP-dependent protein kinase and the disruption of learning in transgenic flies. Neuron, 6(1), 71-82. 
Dubnau, J., Grady, L., Kitamoto, T., \& Tully, T. (2001). Disruption of neurotransmission in Drosophila mushroom body blocks retrieval but not acquisition of memory. Nature, 411(6836), 476-480.

Dudai, Y., Jan, Y. N., Byers, D., Quinn, W. G., \& Benzer, S. (1976). Dunce, a mutant of Drosophila deficient in learning. Proc Natl Acad of Sci, 73(5), 1684-1688.

Dweck, H. K. M., Ebrahim, S. A. M., Kromann, S., Bown, D., Hillbur, Y., Sachse, S., Hansson, B. S., \& Stensmyr, M. C. (2013). Olfactory Preference for Egg Laying on Citrus Substrates in Drosophila. Curr Biol, 23(24), 2472-2480.

Dylla, K. V., Raiser, G., Galizia, C. G., \& Szyszka, P. (2017). Trace Conditioning in Drosophila Induces Associative Plasticity in Mushroom Body Kenyon Cells and Dopaminergic Neurons. Front Neural Circuits, 11, 42.

Ejima, A. (2015). Pleiotropic actions of the male pheromone cis-vaccenyl acetate in Drosophila melanogaster. J Comp Physiol, 201(9), 927-932.

Ejima, A., Smith, B. P. C., Lucas, C., Levine, J. D., \& Griffith, L. C. (2005). Sequential learning of pheromonal cues modulates memory consolidation in trainer-specific associative courtship conditioning. Curr Biol, 15(3), 194-206.

Erber, J., Masuhr, Th., \& Menzel, R. (1980). Localization of short-term memory in the brain of the bee, Apis mellifera. Physiol Entomol, 5(4), 343-358.

Felsenberg, J., Jacob, P. F., Walker, T., Barnstedt, O., Edmondson-Stait, A. J., Pleijzier, M. W., Otto, N., Schlegel, P., Sharifi, N., Perisse, E., Smith, C. S., Lauritzen, J. S., Costa, M., Jefferis, G. S. X. E., Bock, D. D., \& Waddell, S. (2018). Integration of Parallel Opposing Memories Underlies Memory Extinction. Cell, 175(3), 709-722.

Fiala, A., Suska, A., \& Schlüter, O. M. (2010). Optogenetic approaches in neuroscience. Curr Biol, 20(20), R897-903.

Gervasi, N., Tchénio, P., \& Preat, T. (2010). PKA Dynamics in a Drosophila Learning Center: Coincidence Detection by Rutabaga Adenylyl Cyclase and Spatial Regulation by Dunce Phosphodiesterase. Neuron, 65(4), 516-529. 
Griffith, L. C., \& Ejima, A. (2009). Multimodal Sensory Integration of Courtship Stimulating Cues in Drosophila melanogaster. Ann N Y Acad Sci, 1170, 394-398.

Grima, B., Chélot, E., Xia, R., \& Rouyer, F. (2004). Morning and evening peaks of activity rely on different clock neurons of the Drosophila brain. Nature, 431(7010), 869-873.

Hamada, F. N., Rosenzweig, M., Kang, K., Pulver, S. R., Ghezzi, A., Jegla, T. J., \& Garrity, P. A. (2008). An internal thermal sensor controlling temperature preference in Drosophila. Nature, 454(7201), 217-220.

Hancock, C. E., Bilz, F., \& Fiala, A. (2019). In Vivo Optical Calcium Imaging of Learning-Induced Synaptic Plasticity in Drosophila melanogaster. J Vis Exp, 152, e60288.

Hancock, C. E., Geurten, B. R. H., \& Fiala, A. (2020). Visualization of naive and learned odor representations using in vivo calcium imaging and immunohistochemical bouton mapping of single Drosophila mushroom body neurons. STAR Protoc, 1(3), 100210.

Heisenberg, M., Borst, A., Wagner, S., \& Byers, D. (1985). Drosophila mushroom body mutants are deficient in olfactory learning. J Neurogenet, 2(1), 1-30.

Heisenberg, M. (2003). Mushroom body memoir: From maps to models. Nat Rev Neurosci, 4(4), 266275.

Hige, T., Aso, Y., Modi, M. N., Rubin, G. M., \& Turner, G. C. (2015). Heterosynaptic Plasticity Underlies Aversive Olfactory Learning in Drosophila. Neuron, 88(5), 985-998.

Hige, T., Aso, Y., Rubin, G. M., \& Turner, G. C. (2015). Plasticity-driven individualization of olfactory coding in mushroom body output neurons. Nature. 526, 258-262.

Hilfiker, S., Pieribone, V. A., Czernik, A. J., Kao, H. T., Augustine, G. J., \& Greengard, P. (1999). Synapsins as regulators of neurotransmitter release. Philos Trans R Soc Lond B Biol Sci, 354(1381), 269279.

Isabel, G., Pascual, A., \& Preat, T. (2004). Exclusive Consolidated Memory Phases in Drosophila. Science, 304(5673), 1024-1027. 
Ito, H., Fujitani, K., Usui, K., Shimizu-Nishikawa, K., Tanaka, S., \& Yamamoto, D. (1996). Sexual orientation in Drosophila is altered by the satori mutation in the sex-determination gene fruitless that encodes a zinc finger protein with a BTB domain. Proc Natl Acad Sci 93(18), 96879692.

Ito, K., Suzuki, K., Estes, P., Ramaswami, M., Yamamoto, D., \& Strausfeld, N. J. (1998). The organization of extrinsic neurons and their implications in the functional roles of the mushroom bodies in Drosophila melanogaster Meigen. Learn Mem, 5(1), 52-77.

Jefferis, G. S. X. E., Potter, C. J., Chan, A. M., Marin, E. C., Rohlfing, T., Maurer, C. R., \& Luo, L. (2007). Comprehensive Maps of Drosophila Higher Olfactory Centers: Spatially Segregated Fruit and Pheromone Representation. Cell, 128(6), 1187-1203.

Kandel, E. R., Frazier, W. T., \& Coggeshall, R. E. (1967). Opposite Synaptic Actions Mediated by Different Branches of an Identifiable Interneuron in Aplysia. Science, 155(3760), 346-349.

Kandel, E. R., \& Tauc, L. (1965). Heterosynaptic facilitation in neurones of the abdominal ganglion of Aplysia depilans. Journal Physiol, 181(1), 1-27.

Kim, Y.-C., Lee, H.-G., \& Han, K.-A. (2007). D1 Dopamine Receptor dDA1 Is Required in the Mushroom Body Neurons for Aversive and Appetitive Learning in Drosophila. J Neurosci, 27(29), 76407647.

Kitamoto, T. (2001). Conditional modification of behavior in Drosophila by targeted expression of a temperature-sensitive shibire allele in defined neurons. J Neurobiol, 47(2), 81-92.

Konopka, R. J., \& Benzer, S. (1971). Clock mutants of Drosophila melanogaster. Proc Natl Acad Sci, 68(9), 2112-2116.

Krashes, M. J., DasGupta, S., Vreede, A., White, B., Armstrong, J. D., \& Waddell, S. (2009). A neural circuit mechanism integrating motivational state with memory expression in Drosophila. Cell, 139(2), 416-427.

Kuo, S.-Y., Wu, C.-L., Hsieh, M.-Y., Lin, C.-T., Wen, R.-K., Chen, L.-C., Chen, Y.-H., Yu, Y.-W., Wang, H.D., Su, Y.-J., Lin, C.-J., Yang, C.-Y., Guan, H.-Y., Wang, P.-Y., Lan, T.-H., \& Fu, T.-F. (2015). PPL2ab 
neurons restore sexual responses in aged Drosophila males through dopamine. Nat Commun, 6, 7490.

Lai, S.-L., \& Lee, T. (2006). Genetic mosaic with dual binary transcriptional systems in Drosophila. Nat Neurosci, 9(5), 703-709.

Landayan, D., Feldman, D. S., \& Wolf, F. W. (2018). Satiation state-dependent dopaminergic control of foraging in Drosophila. Sci Rep, 8(1), 1-9.

Larsson, M. C., Domingos, A. I., Jones, W. D., Chiappe, M. E., Amrein, H., \& Vosshall, L. B. (2004). Or83b Encodes a Broadly Expressed Odorant Receptor Essential for Drosophila Olfaction. Neuron, 43(5), 703-714.

Lebreton, S., Becher, P. G., Hansson, B. S., \& Witzgall, P. (2012). Attraction of Drosophila melanogaster males to food-related and fly odours. J Insect Physiol, 58(1), 125-129.

Lee, T., \& Luo, L. (1999). Mosaic analysis with a repressible cell marker for studies of gene function in neuronal morphogenesis. Neuron, 22(3), 451-461.

Leiss, F., Groh, C., Butcher, N. J., Meinertzhagen, I. A., \& Tavosanis, G. (2009). Synaptic organization in the adult Drosophila mushroom body calyx. J Comp Neurol,

Lewis, L. P. C., Siju, K. P., Aso, Y., Friedrich, A. B., Bulteel, A. J. B., Rubin, G. M., \& Grunwald Kadow, I. C. (2015). A Higher Brain Circuit for Immediate Integration of Conflicting Sensory Information in Drosophila. Curr Biol, 25(17), 2203-2214.

Li, F., Lindsey, J. W., Marin, E. C., Otto, N., Dreher, M., Dempsey, G., Stark, I., Bates, A. S., Pleijzier, M. W., Schlegel, P., Nern, A., Takemura, S., Eckstein, N., Yang, T., Francis, A., Braun, A., Parekh, R., Costa, M., Scheffer, L. K., ... Rubin, G. M. (2020). The connectome of the adult Drosophila mushroom body provides insights into function. ELife, 9, e62576.

Lim, J., Fernandez, A. I., Hinojos, S. J., Aranda, G. P., James, J., Seong, C. -S., \& Han, K. -A. (2018). The mushroom body D1 dopamine receptor controls innate courtship drive. Genes Brain Behav, 17(2), 158-167. 
Lin, A. C., Bygrave, A., de Calignon, A., Lee, T., \& Miesenböck, G. (2014). Sparse, Decorrelated Odor Coding in the Mushroom Body Enhances Learned Odor Discrimination. Nat Neurosci, 17(4), $559-568$.

Lin, C.-C., Prokop-Prigge, K. A., Preti, G., \& Potter, C. J. (2015). Food odors trigger Drosophila males to deposit a pheromone that guides aggregation and female oviposition decisions. ELife, 4, e08688.

Liu, C., Plaąais, P. Y., Yamagata, N., Pfeiffer, B. D., Aso, Y., Friedrich, A. B., Siwanowicz, I., Rubin, G. M., Preat, T., \& Tanimoto, H. (2012). A subset of dopamine neurons signals reward for odour memory in Drosophila. Nature, 488(7412), 512-516.

Liu, X., \& Davis, R. L. (2009). The GABAergic anterior paired lateral neuron suppresses and is suppressed by olfactory learning. Nat Neurosci, 12(1), 53-59.

Livingstone, M. S., Sziber, P. P., \& Quinn, W. G. (1984). Loss of calcium/calmodulin responsiveness in adenylate cyclase of rutabaga, a Drosophila learning mutant. Cell, 37(1), 205-215.

Louis, T., Stahl, A., Boto, T., \& Tomchik, S. M. (2018). Cyclic AMP-dependent plasticity underlies rapid changes in odor coding associated with reward learning. Proc Natl Acad Sci, 115(3), E448E457.

Luan, H., Peabody, N. C., Vinson, C. R., \& White, B. H. (2006). Refined spatial manipulation of neuronal function by combinatorial restriction of transgene expression. Neuron, 52(3), 425-436.

Lüdke, A., Raiser, G., Nehrkorn, J., Herz, A. V. M., Galizia, C. G., \& Szyszka, P. (2018). Calcium in Kenyon Cell Somata as a Substrate for an Olfactory Sensory Memory in Drosophila. Front Cell Neurosci, 12,128

Mao, Z., \& Davis, R. L. (2009). Eight different types of dopaminergic neurons innervate the Drosophila mushroom body neuropil: Anatomical and physiological heterogeneity. Front Neural Circuits, $3,5$.

Mariath, H. A. (1985). Operant conditioning in Drosophila melanogaster wild-type and learning mutants with defects in the cyclic AMP metabolism. J Insect Physiol, 31(10), 779-787. 
McGuire, S. E., Le, P. T., \& Davis, R. L. (2001). The role of Drosophila mushroom body signaling in olfactory memory. Science, 293(5533), 1330-1333.

McGuire, Sean E., Le, P. T., Osborn, A. J., Matsumoto, K., \& Davis, R. L. (2003). Spatiotemporal rescue of memory dysfunction in Drosophila. Science, 302(5651), 1765-1768.

Menzel, R., \& Erber, J. (1978). Learning and memory in bees. Sci. Am, 239(1), 102-110.

Michels, B., Diegelmann, S., Tanimoto, H., Schwenkert, I., Buchner, E., \& Gerber, B. (2005). A role for Synapsin in associative learning: The Drosophila larva as a study case. Learn Mem, 12(3), 224231.

Morgan, T. H. (1911). An attempt to analyze the constitution of the chromosomes on the basis of sexlimited inheritance in Drosophila. J Exp Zool, 11(4), 365-413.

Morgan, Thomas Hunt. (1910). Chromosomes and heredity. The American Naturalist, 44(524), 449496.

Nakai, J., Ohkura, M., \& Imoto, K. (2001). A high signal-to-noise Ca2+ probe composed of a single green fluorescent protein. Nat Biotechnol, 19(2), 137-141.

Ng, M., Roorda, R. D., Lima, S. Q., Zemelman, B. V., Morcillo, P., \& Miesenböck, G. (2002). Transmission of Olfactory Information between Three Populations of Neurons in the Antennal Lobe of the Fly. Neuron, 36(3), 463-474.

Nitabach, M. N., Blau, J., \& Holmes, T. C. (2002). Electrical Silencing of Drosophila Pacemaker Neurons Stops the Free-Running Circadian Clock. Cell, 109(4), 485-495.

Owald, D., Felsenberg, J., Talbot, C. B., Das, G., Perisse, E., Huetteroth, W., \& Waddell, S. (2015). Activity of defined mushroom body output neurons underlies learned olfactory behavior in Drosophila. Neuron, 86(2), 417-427.

Pascual, A., \& Préat, T. (2001). Localization of long-term memory within the Drosophila mushroom body. Science, 294(5544), 1115-1117.

Pavlov, I. P. (1927). Conditioned reflexes. An Investigation of the Physiological Activity of the Cerebral Cortex. 
Pech, U., Revelo, N. H., Seitz, K. J., Rizzoli, S. O., \& Fiala, A. (2015). Optical Dissection of ExperienceDependent Pre- and Postsynaptic Plasticity in the Drosophila Brain. Cell Rep, 10(12), 20832095.

Perisse, E., Owald, D., Barnstedt, O., Talbot, C. B. B., Huetteroth, W., \& Waddell, S. (2016). Aversive Learning and Appetitive Motivation Toggle Feed-Forward Inhibition in the Drosophila Mushroom Body. Neuron, 90(5), 1086-1099.

Piper, M. D., Blanc, E., Leitão-Gonçalves, R., Yang, M., He, X., Linford, N. J., Hoddinott, M. P., Hopfen, C., Soultoukis, G. A., Niemeyer, C., Kerr, F., Pletcher, S. D., Ribeiro, C., \& Partridge, L. (2014). A holidic medium for Drosophila melanogaster. Nat Methods, 11(1).

Pitman, J. L., Huetteroth, W., Burke, C. J., Krashes, M. J., Lai, S. L., Lee, T., \& Waddell, S. (2011). A pair of inhibitory neurons are required to sustain labile memory in the Drosophila mushroom body. Curr Biol, 21(10), 855-861.

Quinn, W. G., Harris, W. A., \& Benzer, S. (1974). Conditioned behavior in Drosophila melanogaster. Proc Natl Acad Sci, 71(3), 708-712.

Reza, Md. A., Mhatre, S. D., Morrison, J. C., Utreja, S., Saunders, A. J., Breen, D. E., \& Marenda, D. R. (2013). Automated analysis of courtship suppression learning and memory in Drosophila melanogaster. Fly, 7(2), 105-111.

Rideout, E. J., Dornan, A. J., Neville, M. C., Eadie, S., \& Goodwin, S. F. (2010). Control of Sexual Differentiation and Behavior by the doublesex gene in Drosophila melanogaster. Nat Neurosci, 13(4), 458-466.

Riemensperger, T., Issa, A.-R., Pech, U., Coulom, H., Nguyễn, M.-V., Cassar, M., Jacquet, M., Fiala, A., \& Birman, S. (2013). A Single Dopamine Pathway Underlies Progressive Locomotor Deficits in a Drosophila Model of Parkinson Disease. Cell Rep, 5(4), 952-960.

Riemensperger, T., Völler, T., Stock, P., Buchner, E., \& Fiala, A. (2005). Punishment prediction by dopaminergic neurons in Drosophila. Curr Biol, 15(21), 1953-1960. 
Robertson, H. M., Warr, C. G., \& Carlson, J. R. (2003). Molecular evolution of the insect chemoreceptor gene superfamily in Drosophila melanogaster. Proc Natl Acad Sci, 100, 14537-14542.

Root, C. M., Ko, K. I., Jafari, A., \& Wang, J. W. (2011). Presynaptic Facilitation by Neuropeptide Signaling Mediates Odor-Driven Food Search. Cell, 145(1), 133-144.

Ryner, L. C., Goodwin, S. F., Castrillon, D. H., Anand, A., Villella, A., Baker, B. S., Hall, J. C., Taylor, B. J., \& Wasserman, S. A. (1996). Control of male sexual behavior and sexual orientation in Drosophila by the fruitless gene. Cell, 87(6), 1079-1089.

Sachse, S., \& Beshel, J. (2016). The good, the bad, and the hungry: How the central brain codes odor valence to facilitate food approach in Drosophila. Curr Opin Neurobiol, 40, 53-58.

Scheffer, L. K., Xu, C. S., Januszewski, M., Lu, Z., Takemura, S., Hayworth, K. J., Huang, G. B., Shinomiya, K., Maitlin-Shepard, J., Berg, S., Clements, J., Hubbard, P. M., Katz, W. T., Umayam, L., Zhao, T., Ackerman, D., Blakely, T., Bogovic, J., Dolafi, T., ... Plaza, S. M. (2020). A connectome and analysis of the adult Drosophila central brain. ELife, 9, e57443.

Schroll, C., Riemensperger, T., Bucher, D., Ehmer, J., Völler, T., Erbguth, K., Gerber, B., Hendel, T., Nagel, G., Buchner, E., \& others. (2006). Light-induced activation of distinct modulatory neurons triggers appetitive or aversive learning in Drosophila larvae. Curr Biol, 16(17), 17411747.

Schwaerzel, M., Heisenberg, M., \& Zars, T. (2002). Extinction Antagonizes Olfactory Memory at the Subcellular Level. Neuron, 35(5), 951-960.

Scott, K., Brady, R., Cravchik, A., Morozov, P., Rzhetsky, A., Zuker, C., \& Axel, R. (2001). A Chemosensory Gene Family Encoding Candidate Gustatory and Olfactory Receptors in Drosophila. Cell, 104(5), 661-673.

Semon, R. W. (1921). The mneme. London : Allen \& Unwin.

Siegel, R. W., \& Hall, J. C. (1979). Conditioned responses in courtship behavior of normal and mutant Drosophila. Proc Natl Acad Sci, 76(7), 3430-3434.

Skinner, B. F. (1948). 'Superstition' in the pigeon. J Exp Psychol, 38(2), 168-172. 
Skinner, Burrhus Frederic. (1938). The behaviour of organisms: An experimental analysis. D. AppletonCentury Company Incorporated.

Sturtevant, A. H. (1915). Experiments on sex recognition and the problem of sexual selection in Drosophila. J Anim Behav, 5(5), 351-366.

Suh, G. S. B., Ben-Tabou de Leon, S., Tanimoto, H., Fiala, A., Benzer, S., \& Anderson, D. J. (2007). Light Activation of an Innate Olfactory Avoidance Response in Drosophila. Curr Biol, 17(10), 905908.

Takemura, S. ya, Aso, Y., Hige, T., Wong, A., Lu, Z., Xu, C. S., Rivlin, P. K., Hess, H., Zhao, T., Parag, T., Berg, S., Huang, G., Katz, W., Olbris, D. J., Plaza, S., Umayam, L., Aniceto, R., Chang, L. A., Lauchie, S., ... Scheffer, L. K. (2017). A connectome of a learning and memory center in the adult Drosophila brain. ELife, 6, 1-43.

Tanaka, N. K., Awasaki, T., Shimada, T., \& Ito, K. (2004). Integration of Chemosensory Pathways in the Drosophila Second-Order Olfactory Centers. Curr Biol, 14(6), 449-457.

Tanaka, N. K., Tanimoto, H., \& Ito, K. (2008). Neuronal assemblies of the Drosophila mushroom body. J Comp Neurol, 508(5), 711-755.

Tempel, B. L., Bonini, N., Dawson, D. R., \& Quinn, W. G. (1983). Reward learning in normal and mutant Drosophila. Proc Natl Acad Sci, 80(5), 1482-1486.

Thévenaz, P., Ruttimann, U. E., \& Unser, M. (1998). A pyramid approach to subpixel registration based on intensity. IEEE Transactions on Image Processing: A Publication of the IEEE Signal Processing Society, 7(1), 27-41.

Tian, L., Hires, S. A., Mao, T., Huber, D., Chiappe, M. E., Chalasani, S. H., Petreanu, L., Akerboom, J., McKinney, S. A., Schreiter, E. R., Bargmann, C. I., Jayaraman, V., Svoboda, K., \& Looger, L. L. (2009). Imaging neural activity in worms, flies and mice with improved GCaMP calcium indicators. Nat Methods, 6(12), 875-881. 
Trannoy, S., Penn, J., Lucey, K., Popovic, D., \& Kravitz, E. A. (2016). Short and long-lasting behavioral consequences of agonistic encounters between male Drosophila melanogaster. Proc Natl Acad Sci, 113(17), 4818-4823.

Tsao, C.-H., Chen, C.-C., Lin, C.-H., Yang, H.-Y., \& Lin, S. (2018). Drosophila mushroom bodies integrate hunger and satiety signals to control innate food-seeking behavior. ELife, 7, e35264.

Tully, T., \& Quinn, W. G. (1985). Classical conditioning and retention in normal and mutant Drosophila melanogaster. J Comp Physiol, 157(2), 263-277.

Vosshall, L. B., Wong, A. M., \& Axel, R. (2000). An olfactory sensory map in the fly brain. Cell, 102(2), 147-159.

Wang, Y., Mamiya, A., Chiang, A. -s, \& Zhong, Y. (2008). Imaging of an Early Memory Trace in the Drosophila Mushroom Body. J Neurosci, 28(17), 4368-4376.

Wilson, R. I., Turner, G. C., \& Laurent, G. (2004). Transformation of Olfactory Representations in the Drosophila Antennal Lobe. Science, 303(5656), 366-370.

Wu, J. S., \& Luo, L. (2006). A protocol for mosaic analysis with a repressible cell marker (MARCM) in Drosophila. Nat Protoc, 1(6), 2583-2589.

Wustmann, G., Rein, K., Wolf, R., \& Heisenberg, M. (1996). A new paradigm for operant conditioning of Drosophila melanogaster. J Comp Physiol, 179(3), 429-436.

Yamamoto, D., \& Koganezawa, M. (2013). Genes and circuits of courtship behaviour in Drosophila males. Nat Rev Neurosci, 14(10), 681-692.

Yin, J. C., Vecchio, M. D., Zhou, H., \& Tully, T. (1995). CREB as a memory modulator: Induced expression of a dCREB2 activator isoform enhances long-term memory in Drosophila. Cell, 81(1), 107115.

Yin, J. C., Wallach, J. S., Vecchio, M. D., Wilder, E. L., Zhou, H., Quinn, W. G., \& Tully, T. (1994). Induction of a dominant negative CREB transgene specifically blocks long-term memory in Drosophila. Cell, 79(1), 49-58. 
Yu, D., Akalal, D. B. G., \& Davis, R. L. (2006). Drosophila $\alpha / \beta$ Mushroom Body Neurons Form a BranchSpecific, Long-Term Cellular Memory Trace after Spaced Olfactory Conditioning. Neuron, 52(5), 845-855.

Yurkovic, A., Wang, O., Basu, A. C., \& Kravitz, E. A. (2006). Learning and memory associated with aggression in Drosophila melanogaster. Proc Natl Acad Sci, 103(46), 17519-17524.

Zars, T., Fischer, M., Schulz, R., \& Heisenberg, M. (2000a). Localization of a Short-Term Memory in Drosophila. Science, 288(5466), 672-675.

Zars, T., Wolf, R., Davis, R., \& Heisenberg, M. (2000b). Tissue-Specific Expression of a Type I Adenylyl Cyclase Rescues the rutabaga Mutant Memory Defect: In Search of the Engram. Learn Mem, $7(1), 18-31$.

Zhu, J., Park, K.-C., \& Baker, T. C. (2003). Identification of Odors from Overripe Mango That Attract Vinegar Flies, Drosophila melanogaster. J Chem Ecol, 29(4), 899-909.

Ziegler, A. B., Berthelot-Grosjean, M., \& Grosjean, Y. (2013). The smell of love in Drosophila. Front Physiol, 4, 72. 


\section{Acknowledgements}

I thank my supervisor, Prof. André Fiala, for giving me the opportunity to carry out this work in his workgroup and for always providing guidance and support throughout my time in the lab. I would also like to thank Dr. Camin Dean and Prof. Gregor Bucher for their time and their supportive and encouraging feedback as part of my thesis advisory committee, and Dr. Dean in particular for reviewing this thesis.

I am also grateful to Dr. Thomas Riemensperger and Dr. Florian Bilz for their technical help in learning the calcium imaging procedures, Dr. Bart Geurten for his generous support and discussions, and to Dr. Annekathrin Widmann for her support with statistics, genetics, and for helping me through the writing of this thesis. I also thank all past members of the lab for their support and contributions to discussions of the data presented here.

I am grateful to our collaborators in the Nawrot workgroup at the University of Cologne - Prof. Martin Nawrot, Vahid Rostami, Dr. Hannes Rapp, and Magdalena Springer- for discussions of the data in section 5 .

I am fortunate to have supervised several students who contributed to this work and also to my experience over the last few years - Haiko Poppinga, Maria Woitow, Judith Limley, Tihana Hamzaj, Melanie Stenger, Lena Dorn, and Jenny Henning.

I am especially grateful to Jutta Böker, for keeping the lab (and all its members!) going through everything, and for always making time to help. Likewise, I thank Anne Lattner for her assistance in administrative tasks but more so for always going above and beyond expectations - I have yet to find a problem that she could not fix!

I am grateful to my fellow PhD students - Yazid Rachad, Stephan Deimal, Nora Jahnen, and Hanna Franz - for their companionship and support. I am particularly thankful to Dr. Annekathrin Widmann for vibrant scientific discussions and her contributions to the workgroup - but also personally, for her endless support in and out of the lab, without which all of this would have been a lot harder (and a lot less fun).

Above all else, I am thankful to my mother - for her boundless love and support, and for instilling in me enough compassion, stubbornness, and humour to get me through anything. 


\section{Appendix}

\subsection{Supplemental graphs}

12.1.1 MBONs- $\gamma 2-5$ show no detectable postsynaptic memory trace

Data here show the quantification of odour-evoked responses in $\mathrm{MBON}-\gamma 2-\mathrm{MBON}-\gamma 5$ as seen in Figure 5.4., with the addition of mean calcium traces.
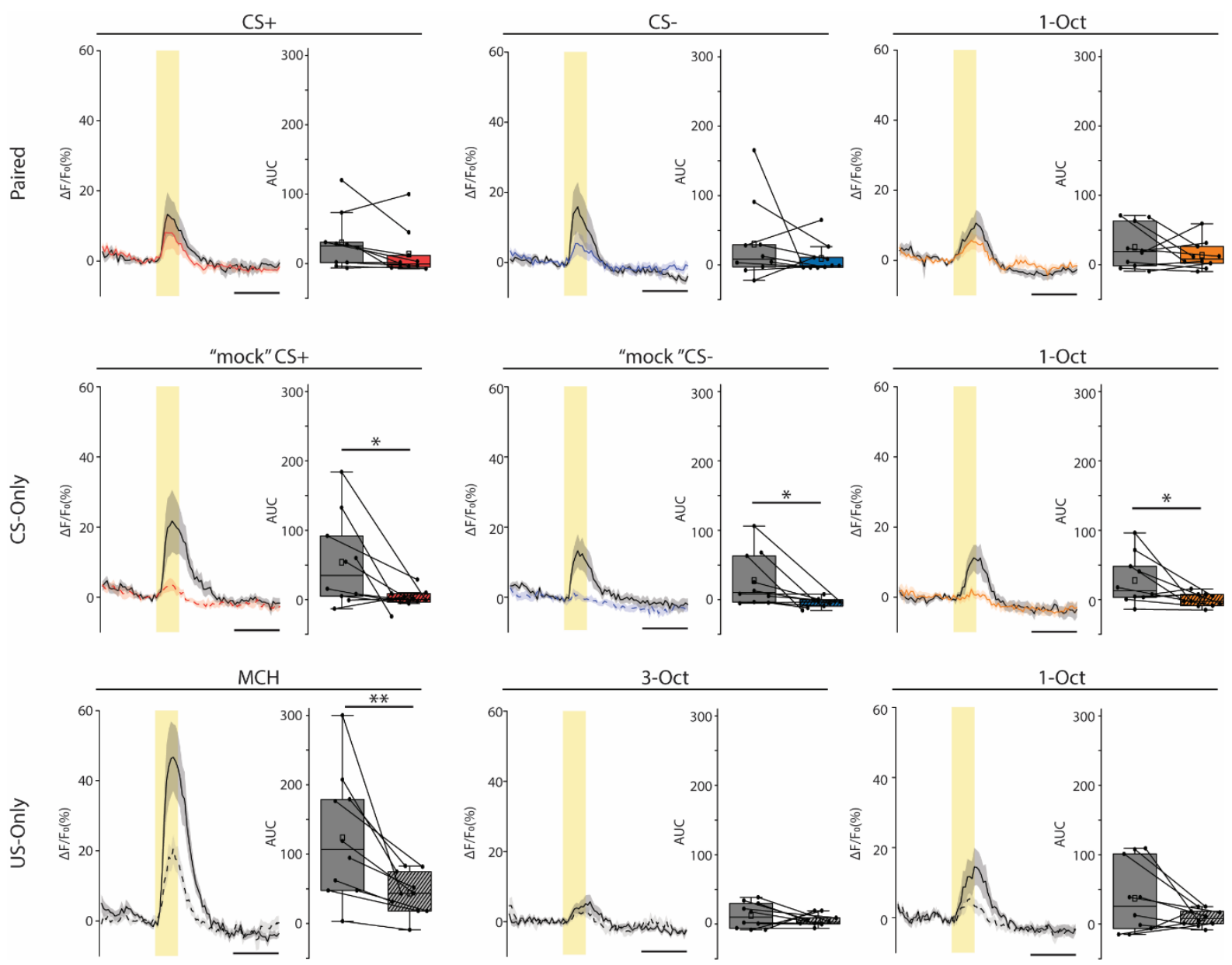

Figure 12.1.: MBON- $\boldsymbol{\gamma} 2$ odour-evoked responses before and after different training regimes. Traces show mean $\triangle F / F_{0}$ over time across animals of the same condition, shaded area indicates SEM. Yellow bar shows odour presentation period. Scale bar represents 5 seconds. Box plots show integrated AUC. Pre-to-post changes in odour responses were tested with a paired Wilcoxon signed ranks test $\left({ }^{*} p<0.05,{ }^{* *} p<0.01\right)$. 

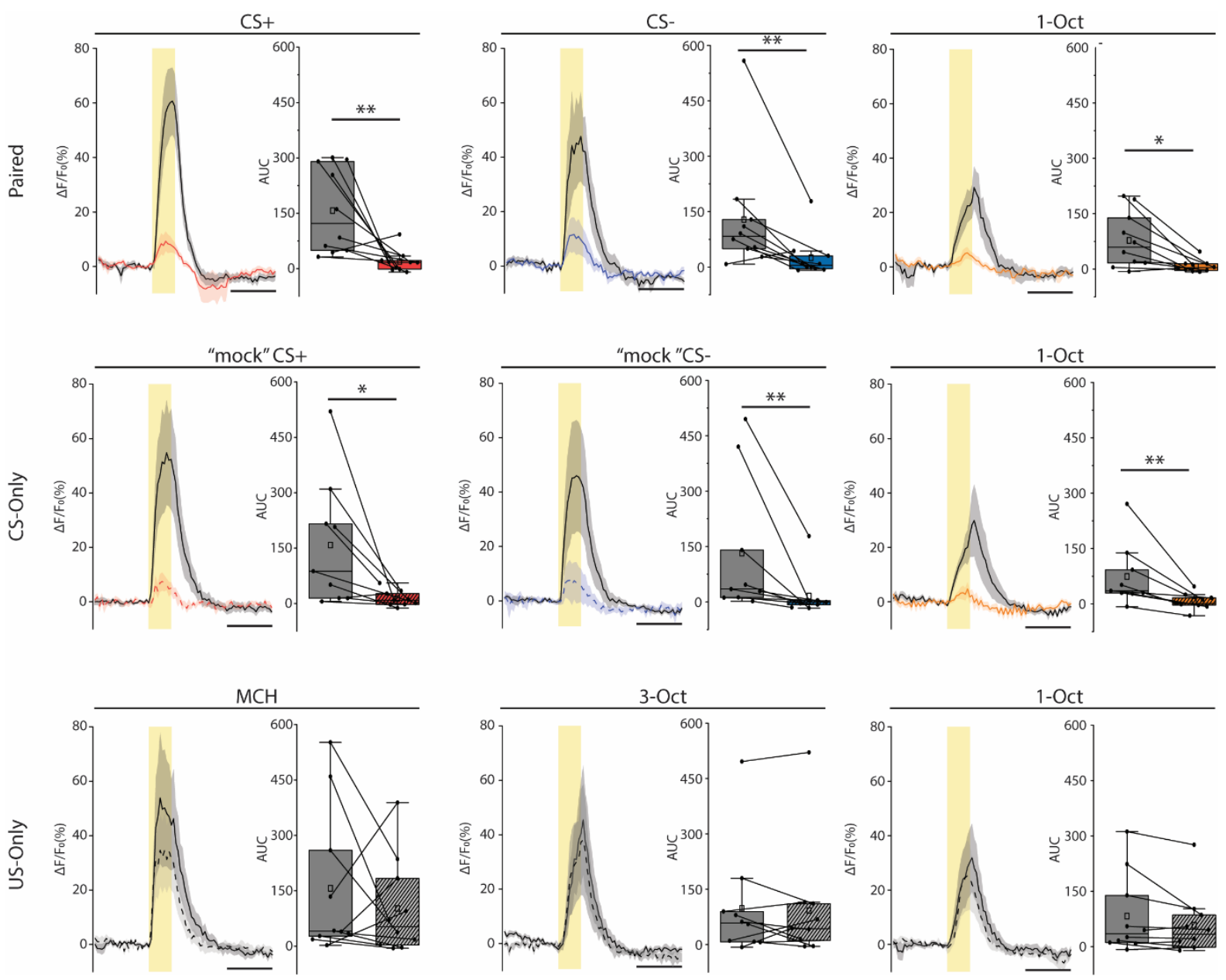

Figure 12.2.: MBON- $\gamma 3$ odour-evoked responses before and after different training regimes. Traces show mean $\Delta F / F_{0}$ over time across animals of the same condition, shaded area indicates SEM. Yellow bar shows odour presentation period. Scale bar represents 5 seconds. Box plots show integrated AUC. Pre-to-post changes in odour responses were tested with a paired Wilcoxon signed ranks test $\left({ }^{*} p<0.05,{ }^{* *} p<0.01\right)$. 

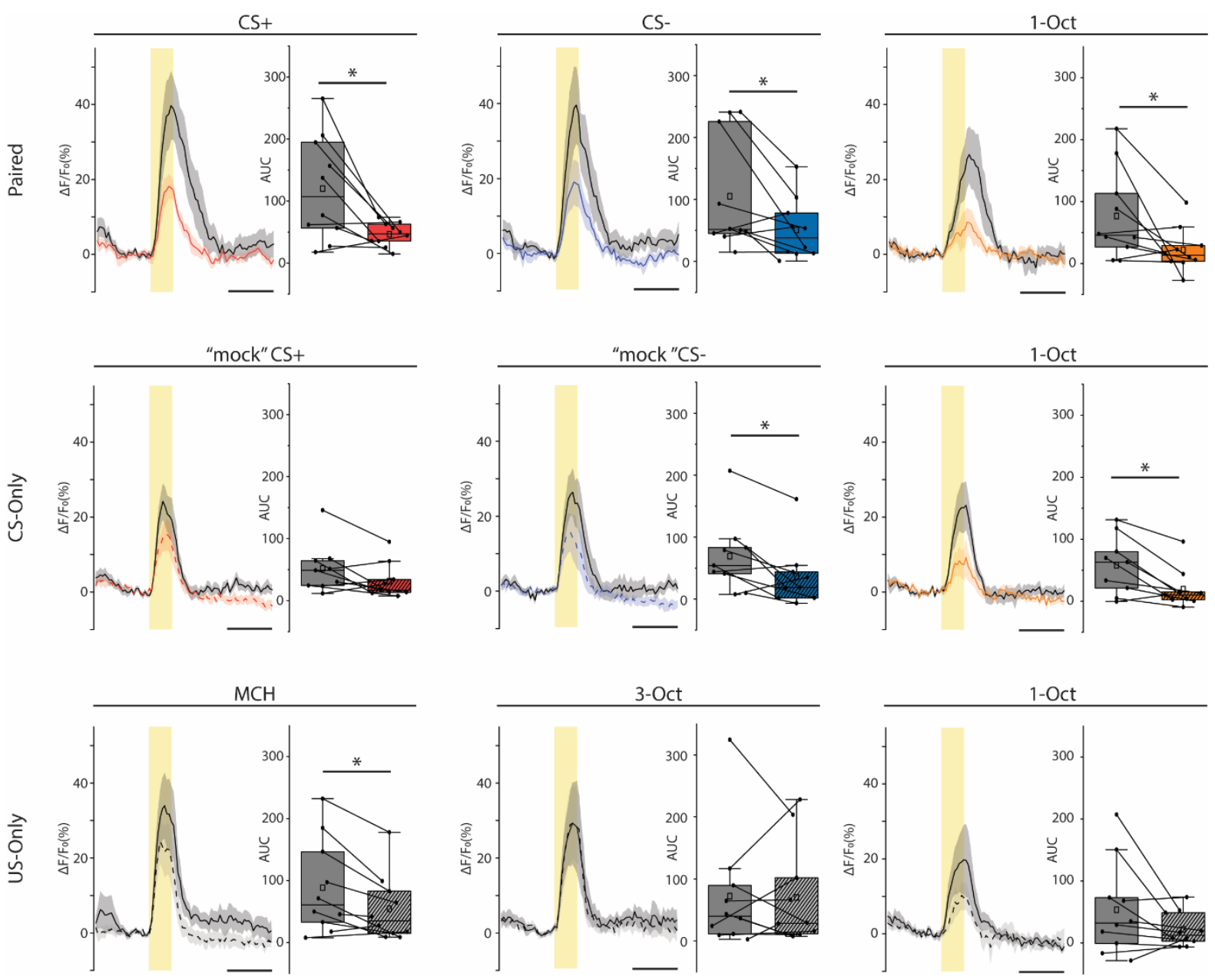

Figure 12.3.: MBON- $\boldsymbol{4}$ odour-evoked responses before and after different training regimes. Traces show mean $\triangle \mathrm{F} / \mathrm{F}_{0}$ over time across animals of the same condition, shaded area indicates SEM. Yellow bar shows odour presentation period. Scale bar represents 5 seconds. Box plots show integrated AUC. Pre-to-post changes in odour responses were tested with a paired Wilcoxon signed ranks test $\left({ }^{*} p<0.05\right)$. 

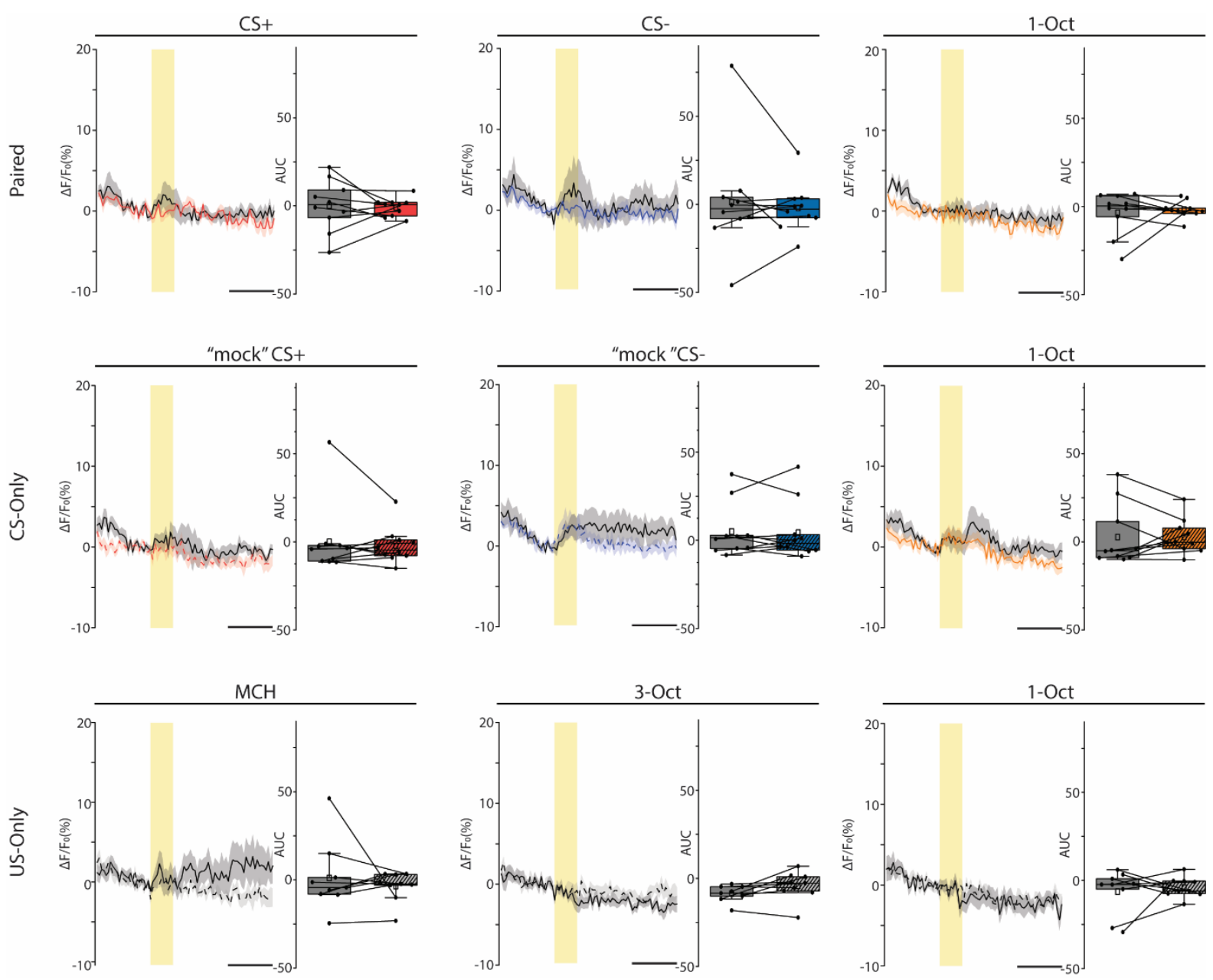

Figure 12.4.: MBON- $\gamma 5$ odour-evoked responses before and after different training regimes. Traces show mean $\Delta F / F_{0}$ over time across animals of the same condition, shaded area indicates SEM. Yellow bar shows odour presentation period. Scale bar represents 5 seconds. Box plots show integrated AUC. Pre-to-post changes in odour responses were tested with a paired Wilcoxon signed ranks test $(* p<0.05)$. 


\subsection{Supplemental material - Visualization of a Distributed Synaptic Memory Code in}

the Drosophila Brain

Supplemental Figure S2 from the publication "Visualization of a Distributed Synaptic Memory Code in the Drosophila Brain"
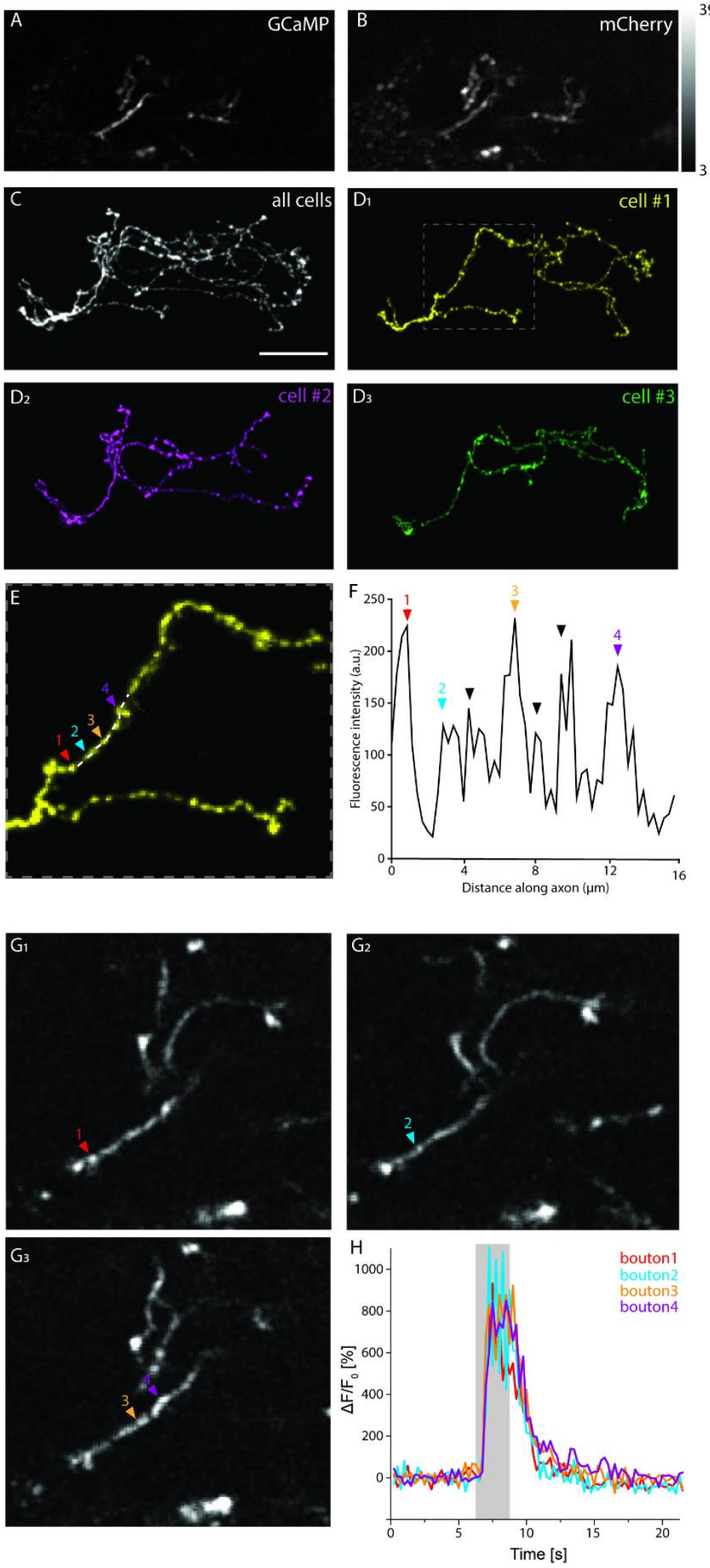

Figure 12.5: Single KC bouton assignment process. (A) and (B) One focal plane used for in vivo measurement of olfactory $\mathrm{Ca} 2+$ responses is shown as an example (same fly as shown in Figure 1, maximum intensity projection over time for each fluorescence channel, one out of six focal planes). (C) Confocal microscopy image after brain removal and immunohistochemistry. In this fly, three KCs expressed transgenes and were disentangled using confocal microscopy image stacks and subsequently 3D reconstructions were generated (see Supplementary Movie 1). Scale bar: $20 \mu \mathrm{m}$. (D1-D3) The three KCs after disentanglement and isolation (cell \#1, yellow; cell \#2 magenta; cell \#3 green). (E) Magnified region of cell \#1 (dashed box in D1). Boutons were identified by their round morphology and increased fluorescence intensity in these structures. Example boutons 1-4 are indicated. (F) Fluorescence intensity along the white dashed line on the axon shown in (E), exemplary of the increased fluorescence intensity at the site of identified boutons. Corresponding boutons to image in (E) are indicated. (G) Mapping of the boutons identified in anatomical images onto the images captured in prior in vivo visualization. Using images as in (A) and (B), along with zstacks captured during imaging, axonal branches and boutons can be mapped between the anatomical and functional KC visualizations, allowing 
for boutons to be assigned to KCs and also to compartments of the $\gamma$-lobe. $(\mathrm{H})$ Responses of the four example boutons to $\mathrm{MCH}$. 
Supplemental Figure S6 from the publication "Visualization of a Distributed Synaptic Memory Code in the Drosophila Brain"

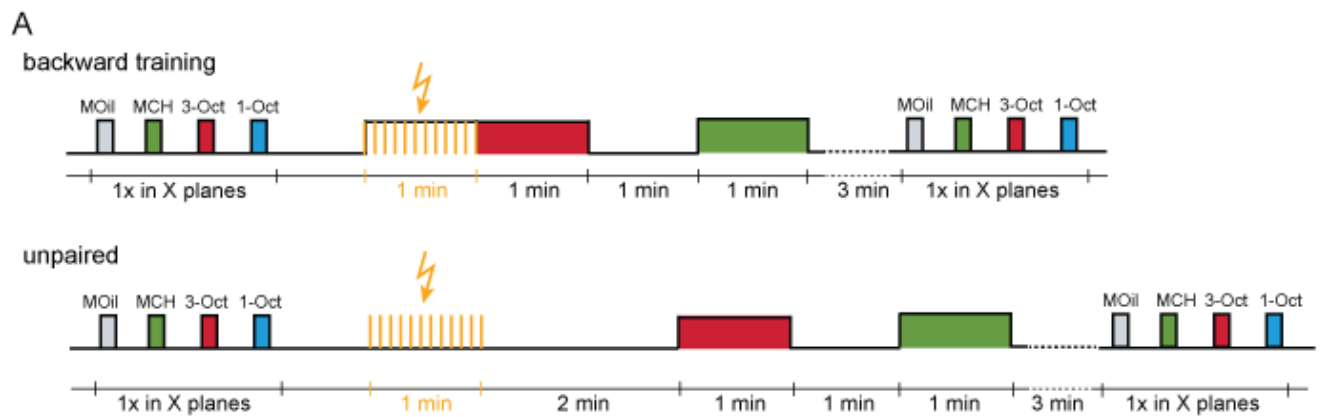

B
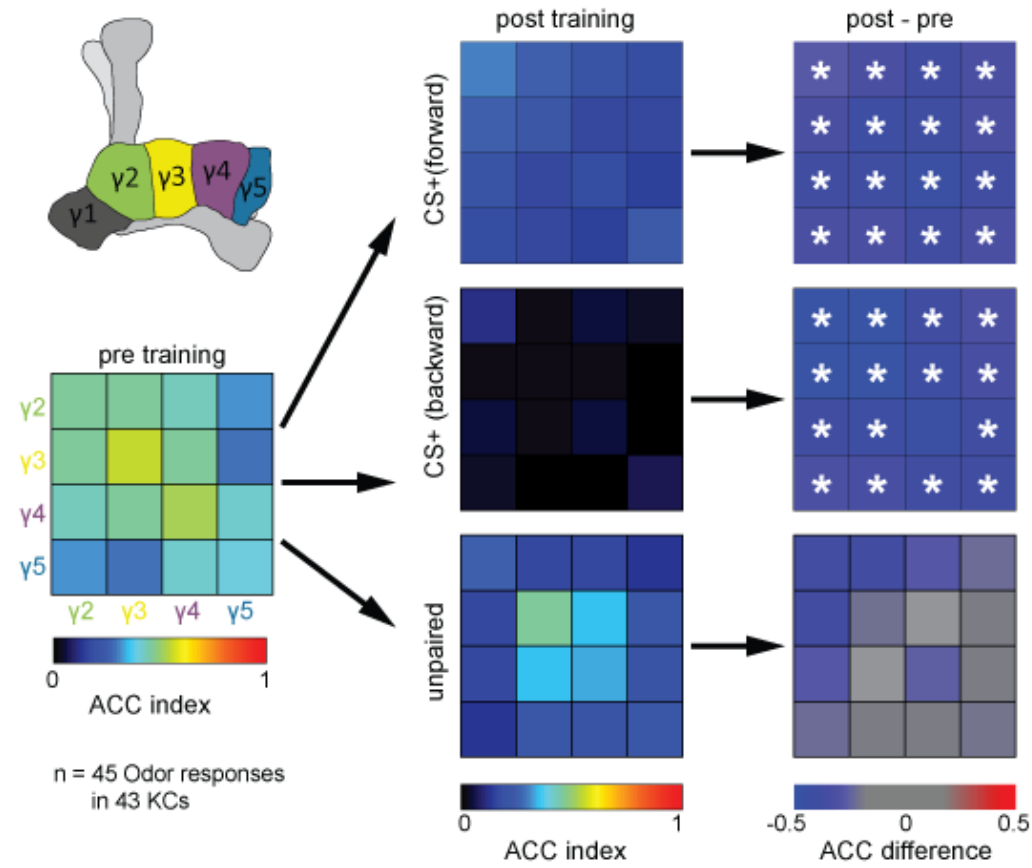

C
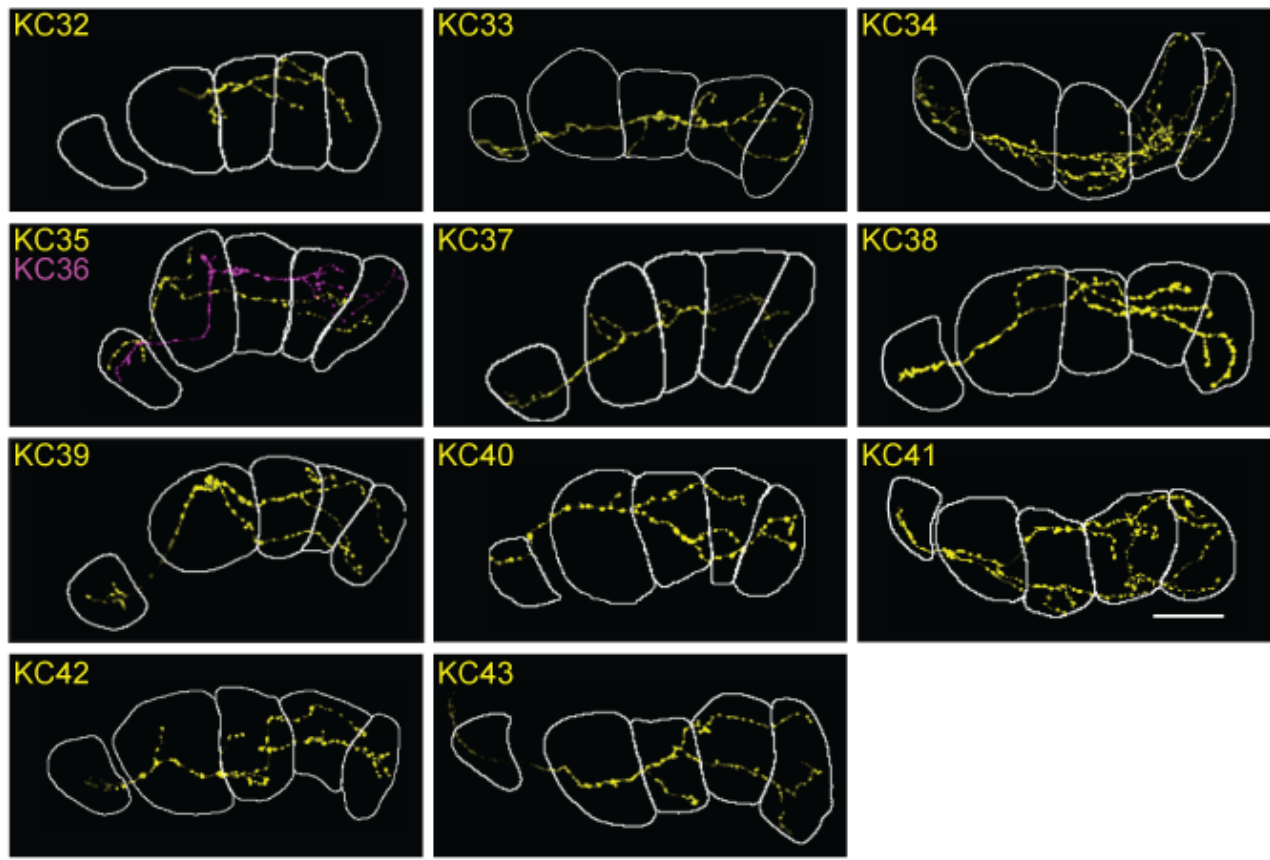
Figure 12.6.: Backward conditioning, but not unpaired stimulation, induce bouton decorrelation across the $\gamma$-lobe. (A) Schematic of the backward conditioning regime. 12 additional KCs were recorded using either backward conditioning or forward conditioning. For simplicity, only one odor combination is shown here. (B) Color-coded amplitude-corrected correlation values (ACC index) for $\mathrm{Ca} 2+$ dynamics of synaptic boutons within each compartment (internal correlation, diagonal) or across compartments. The congruence of $\mathrm{Ca} 2+$ activity within and across compartments decreased after training for $\mathrm{CS}+$ in forward $(n=4)$ and backward $(n=4)$ conditioning, but not for the unpaired condition $(n=4)$. Asterisks indicate statistical significance. For the pre-training situation all KCs recorded in this study were pooled. (C) Reconstruction of the axons of the additional $12 \gamma$-lobe KCs responsive to trained odorants. The white borders indicate the borders of the $\psi$-lobe compartments $(\gamma 1-\gamma 5$, from left to right). Scale bar: $20 \mu \mathrm{m}$. 